Florida International University FIU Digital Commons

$11-4-2016$

\title{
Elucidating The Role of MifS-MifR Two- Component System in Regulating Pseudomonas aeruginosa Pathogenicity
}

Gorakh Digambar Tatke

Florida International University, gorakhdt@gmail.com

DOI: $10.25148 /$ etd.FIDC001220

Follow this and additional works at: https:// digitalcommons.fiu.edu/etd

Part of the Bacteriology Commons, Biology Commons, Microbial Physiology Commons, Pathogenic Microbiology Commons, and the Physiology Commons

\section{Recommended Citation}

Tatke, Gorakh Digambar, "Elucidating The Role of MifS-Mif R Two-Component System in Regulating Pseudomonas aeruginosa Pathogenicity" (2016). FIU Electronic Theses and Dissertations. 3002.

https://digitalcommons.fiu.edu/etd/3002 


\title{
FLORIDA INTERNATIONAL UNIVERSITY
}

\author{
Miami, Florida
}

\section{ELUCIDATING THE ROLE OF MIFS-MIFR TWO-COMPONENT SYSTEM IN REGULATING PSEUDOMONAS AERUGINOSA PATHOGENICITY}

\author{
A dissertation submitted in partial fulfillment of \\ the requirements for the degree of \\ DOCTOR OF PHILOSOPHY \\ in \\ BIOLOGY \\ by \\ Gorakh Digambar Tatke
}


To: Dean Michael R. Heithaus

College of Arts, Sciences and Education

This dissertation, written by Gorakh Digambar Tatke, and entitled Elucidating the Role of MifS-MifR Two-Component System in Regulating Pseudomonas aeruginosa Pathogenicity, having been approved in respect to style and intellectual content, is referred to you for judgment.

We have read this dissertation and recommend that it be approved.

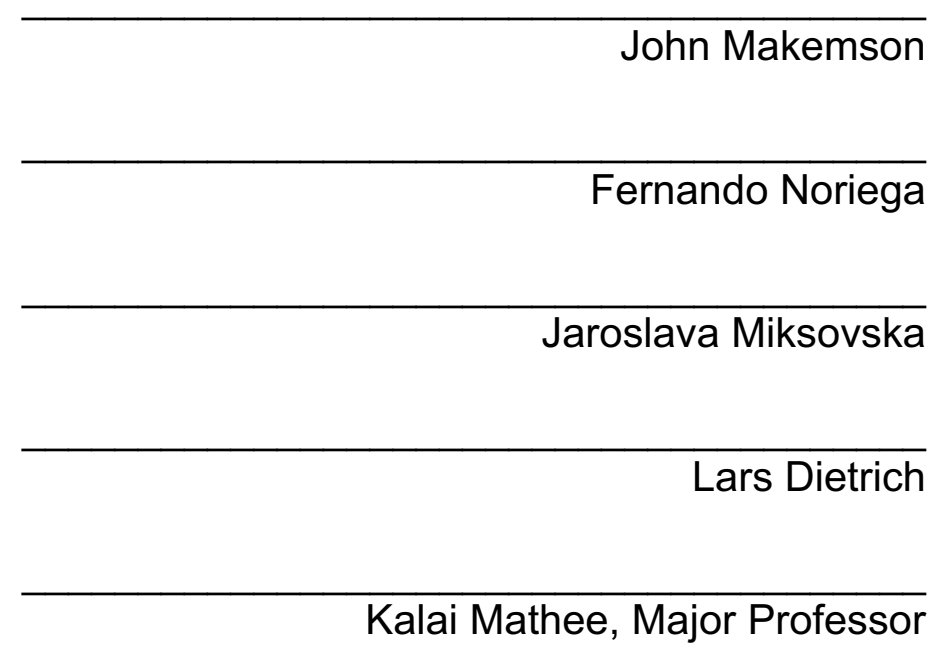

Date of Defense: November 04, 2016

The dissertation of Gorakh Digambar Tatke is approved.

$\begin{array}{r}\text { Dean Michael R. Heithaus } \\ \text { College of Arts, Sciences and Education } \\ \hline \begin{array}{r}\text { Andrès G. Gil } \\ \text { Vice President for Economic Development } \\ \text { and Dean of the University Graduate School }\end{array}\end{array}$

Florida International University, 2016 
(C) Copyright 2016 by Gorakh Digambar Tatke

All rights reserved. 


\section{DEDICATION}

This work is dedicated to my Family, Teachers and Friends for their constant support, guidance and love. 


\section{ACKNOWLEDGMENTS}

This dissertation wouldn't have been possible without the immense support and advice of many people and I am indebted to all of them. I owe my sincere gratitude to my supervisor, Dr. Kalai Mathee, who has always been the guiding force throughout this wonderful Ph.D. journey. I thank her for giving me the opportunity to work on this brilliant project that will always be one of the most important chapters of my life. Her encouragement, guidance and support right from the day I met her in Bangalore for my Ph.D. interview till the end has enabled me to become a better researcher and a human being. It has been a phenomenal learning experiencing under her mentorship and I thank her for allowing me to experience different facets of life, for trusting me with mentoring undergraduate students, working on several collaborative projects, with handling RJS foundation and organizing conferences. I would also like to thank Dr. Giri Narasimhan for his constant support, guidance and for hosting all the wonderful parties. I am also heartily thankful to my committee members, Dr. John Makemson, Dr. Fernando Noriega, Dr. Jaroslava Miksovska and Dr. Lars Dietrich for their guidance and assistance that led me in the right direction of my graduate research. I wish to thank Dr. Giri Narasimhan, Dr. Juan Liuzzi, Dr. Jose Almirall and Dr. Sakhrat Khizroev for giving me the opportunity to work on multiple collaborative projects. I wish to recognize Dr. Eugenia Silva-Herzog, Dr. Lisa Schneper and Dr.Hansi Kumari for their immense help and support throughout my Ph.D. with analytical techniques and sound advice. A special thanks to Celine Murton, Constance Thurmond and Maria Victoria Lorenzo for being amazing students and for putting 
up with me during my qualifying exams and dissertation writing. I would also like to thank all of my present and past laboratory colleagues for always being there for me. I want to express my thanks to all the faculties and staffs in the Department of Biological Sciences and H.W. College of Medicine at Florida International University who continuously supported me with advice and friendship. My special regards to Jitesh K. Pillai for being a wonderful friend for over a decade, for putting up with me with all my scientific ideas, and for his constant motivation, encouragement, advice and friendship during thick and thin. My regards to Parul Maheshwari, Mansi Sharma, Supurna Dhar, Diansy Zincke, Pratik Nyati, Vidya Sagar, Santhosh Narayanan, Deepak Balasubramanian, Kishore Dhavala, Pandiaraj Manickam, Shashank Pawitvar, Ravi Shinde, Jonathan Segal, Zackari Abeyta, Sundar Pandey and Kyle Martins for all the coffees, discussions and importantly for their friendship and peer support.

Lastly, I am grateful to my parents Digambar Tatke, Sandhya Tatke, my sisters Gauri Tatke and Gayatri Tatke for their unwavering support, inspiration and for their faith and confidence in me. 


\title{
ABSTRACT OF THE DISSERTATION \\ ELUCIDATING THE ROLE OF MIFS-MIFR TWO-COMPONENT SYSTEM IN \\ REGULATING PSEUDOMONAS AERUGINOSA PATHOGENICITY
}

\author{
by \\ Gorakh Digambar Tatke \\ Florida International University, 2016 \\ Miami, Florida \\ Professor Kalai Mathee, Major Professor
}

\begin{abstract}
Pseudomonas aeruginosa is a Gram-negative, metabolically versatile, opportunistic pathogen that exhibits a multitude of virulence factors, and is extraordinarily resistant to a gamut of clinically significant antibiotics. This ability is in part mediated by two-component systems (TCS) that play a crucial role in regulating virulence mechanisms, metabolism and antibiotic resistance. Our sequence analysis of the $P$. aeruginosa PAO1 genome revealed the presence of two open reading frames, mifS and mifR, which encodes putative TCS proteins, a histidine sensor kinase MifS and a response regulator MifR, respectively. This twogene operon was found immediately upstream of the poxAB operon, where pox $B$ encodes a chromosomal ß-lactamase, hinting at the role of MifSR TCS in regulating antibiotic resistance. However, loss of mifSR had no effect on the antibiotic resistance profile when compared to $P$. aeruginosa parent PAO1 strain. Subsequently, our phenotypic microarray data (BioLOG) and growth profile studies indicated the inability of mifSR mutants to grow in a-ketoglutarate ( $\alpha-K G$ ), a key
\end{abstract}


tricarboxylic acid (TCA) cycle intermediate, as a sole carbon source. To date, very little is known about the physiology of $P$. aeruginosa when provided with $\alpha-K G$ as its sole carbon source and the role of MifS and MifR TCS in virulence. Importantly, in the recent years, $\alpha-K G$ has gained notoriety for its newly identified role as a signaling molecule in addition to its conventional role in metabolism. This led us to hypothesize that MifSR TCS is involved in $\alpha-K G$ utilization and virulence in $P$. aeruginosa. Using mifS, mifR and mifSR clean in-frame deletion strains, our study demonstrates that the MifSR TCS modulates the expression $P$. aeruginosa kgtP (PA5530) and pcaT (PA0229) genes encoding putative a-KG permeases. In addition, our study shows that the MifSR-regulation of these transporters requires functional sigma factor RpoN $\left(\sigma^{54}\right)$. Loss of mifSR in the presence of $\alpha-K G$, resulted in differential regulation of $P$. aeruginosa key virulence determinants including biofilm formation, motility, cell cytoxicity and the production of pyocyanin and pyoverdine. Involvement of multiple regulators and transporters suggests the presence of an intricate circuitry in the transport of $\alpha-K G$ and its importance in $P$. aeruginosa survival. This is further supported by the a-KG-dependent MifSR regulation of multiple virulence mechanisms. Simultaneous regulation of multiple mechanisms involved in $P$. aeruginosa pathogenesis suggests a complex mechanism of MifSR action. Understanding the physiological cues and regulation would provide a better stratagem to fight often indomitable $P$. aeruginosa infections. 


\section{TABLE OF CONTENTS}

CHAPTER

PAGE

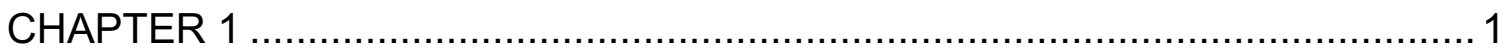

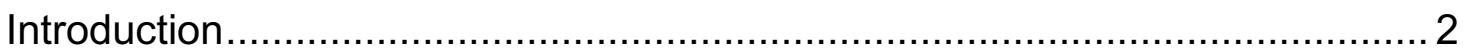

Two Component Regulatory Systems …....................................................... 3

$P$. aeruginosa and two-component systems ............................................... 6

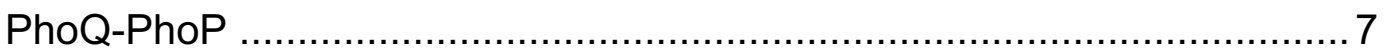

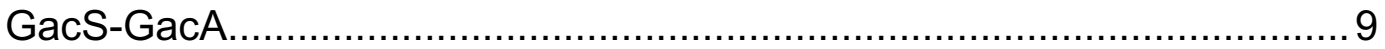

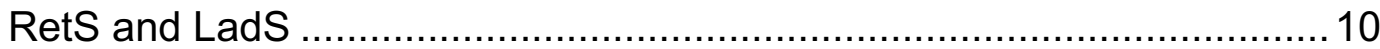

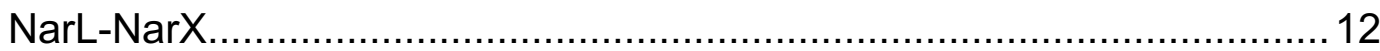

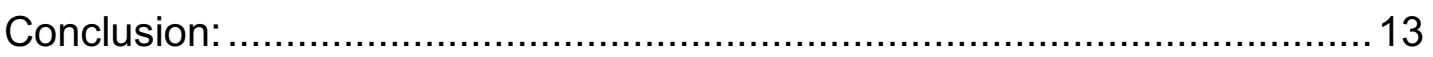

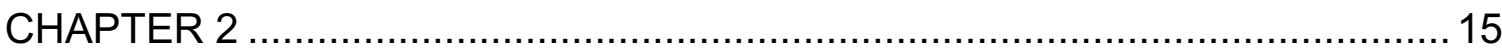

Pseudomonas aeruginosa MifS-MifR two-component system is specific for

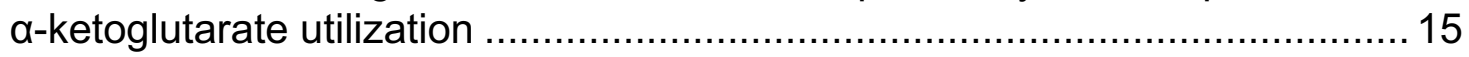

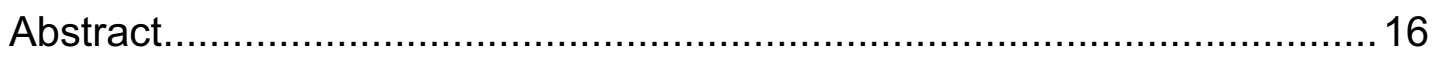

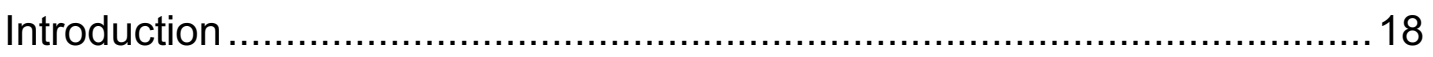

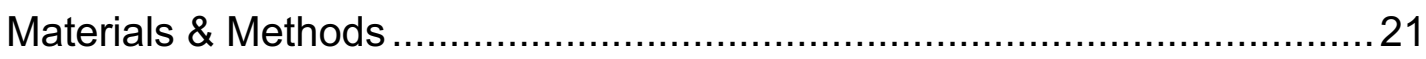

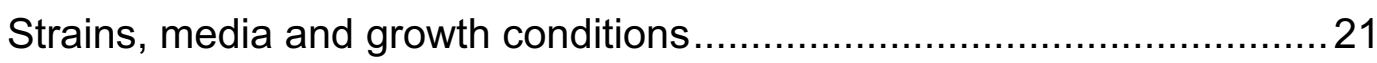

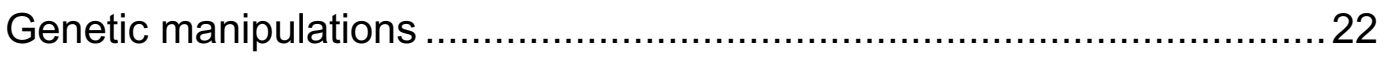

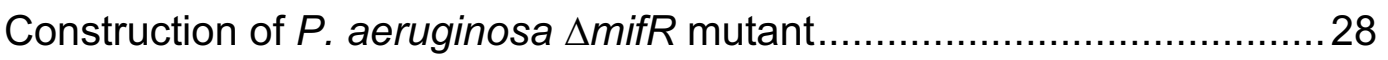

Construction of $P$. aeruginosa $\Delta$ mifS and $\Delta$ mifSR mutants...................28

Construction of complementing plasmids ............................................. 30

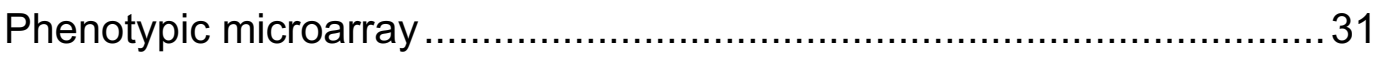

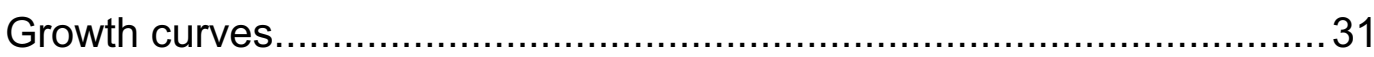

Pyocyanin and pyoverdine production............................................... 32

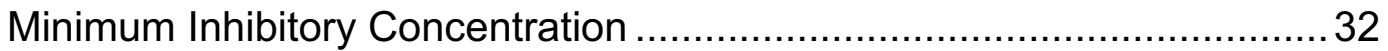

RNA isolation, cDNA synthesis and qRT-PCR ..................................... 33

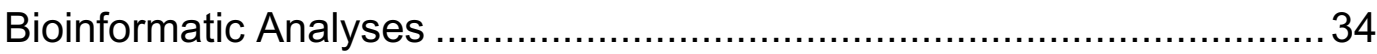

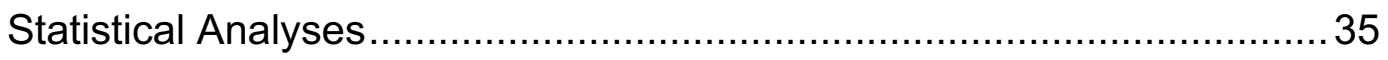

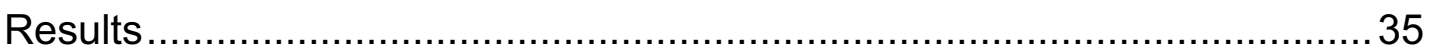

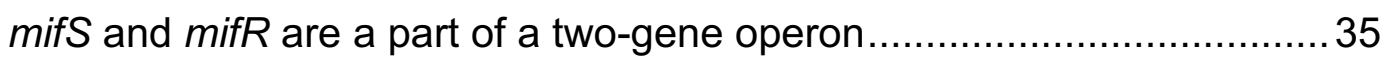


Loss of mifS and mifR did not affect antibiotic resistance 35 mifS, mifR and mifSR mutants failed to grow in the presence of $\alpha-K G$.... 39 mifSR mutants exhibit $\alpha-K G$ dependent growth defect...........................46 mifSR mutants are defective in $\alpha-K G$ transport .....................................50 mifSR TCS genes regulate extracellular $\alpha-K G$ transport.........................52

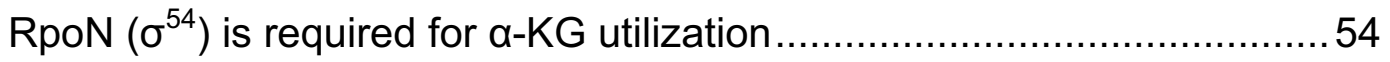

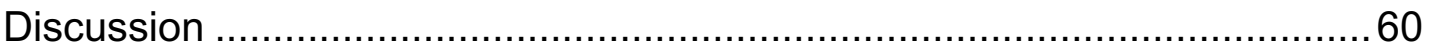

$P$. aeruginosa antibiotic resistance is independent of MifSR TCS ..........60

MifSR TCS regulates $P$. aeruginiosa $\alpha-K G$ utilization ............................61

MifSR TCS modulates $P$. aeruginosa $\alpha-K G$ transport ............................. 64

$P$. aeruginosa $\alpha-K G$ transport requires functional $\operatorname{RpoN}\left(\sigma^{54}\right) \ldots \ldots \ldots \ldots \ldots . . .65$

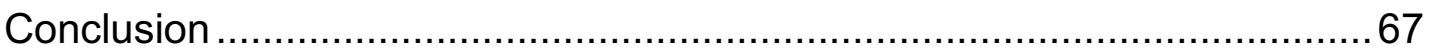

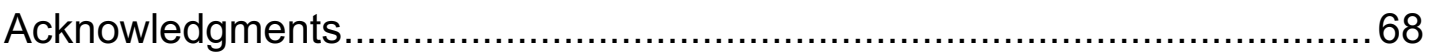

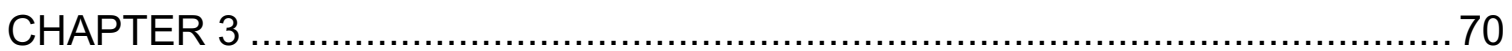

Contemporary role for the ancient metabolite $\alpha$-ketoglutarate in regulating Pseudomonas aeruginosa pathogenicity ................................................. 70

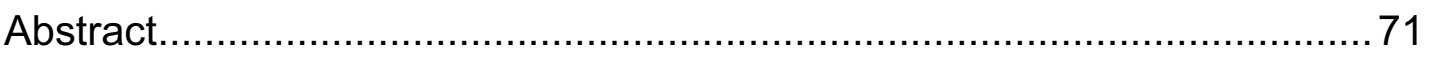

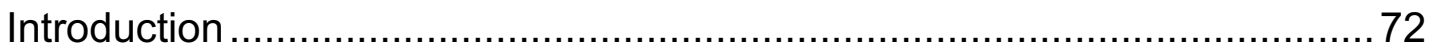

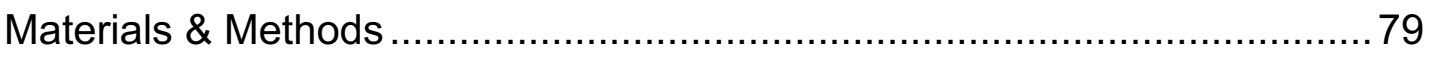

Bacterial strains, media and growth conditions …............................... 79

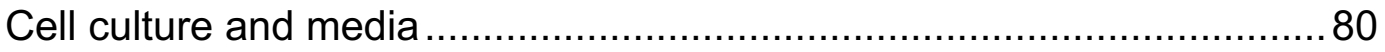

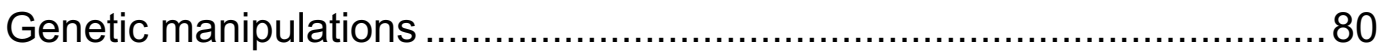

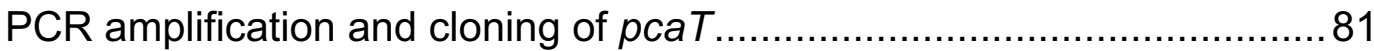

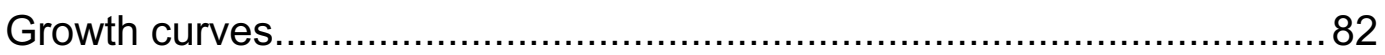

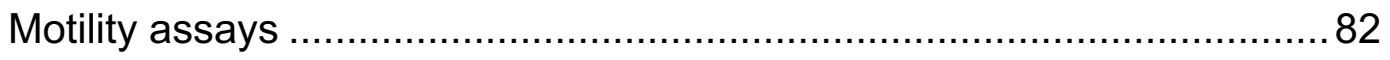

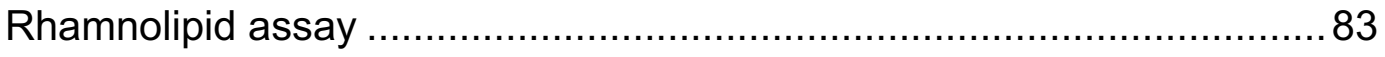

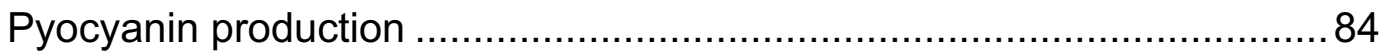

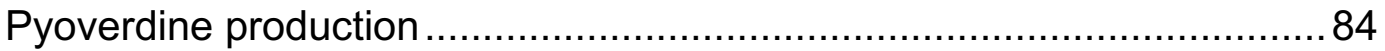

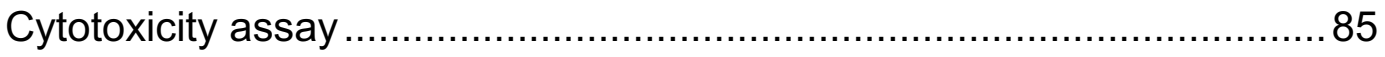

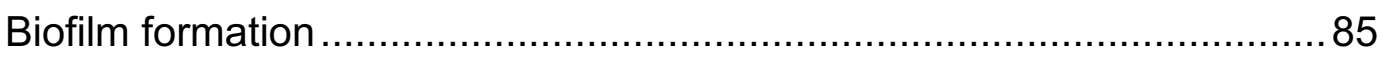

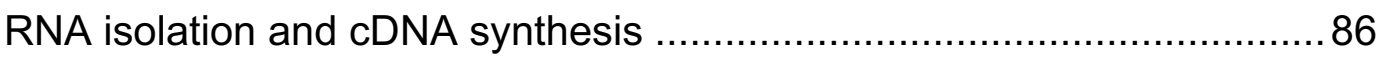




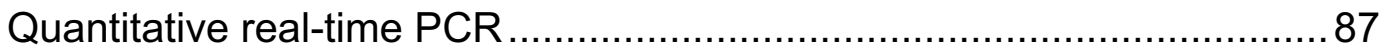

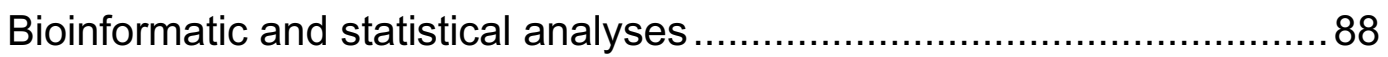

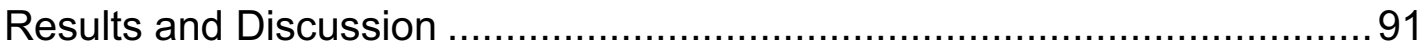

$P$. aeruginosa possess multiple $\alpha-K G$ transport proteins ........................ 91

MifS/MifR TCS regulates $P$. aeruginosa virulence ................................ 99

Enhanced biofilm formation in the absence of mifSR ........................... 100

mifSR deletion mutants are defective in swarming motility ................... 104

Pyocyanin production is negatively regulated by mifSR ........................110

mifSR positively regulates pyoverdine production .............................. 115

Loss of mifR increases cell cytotoxicity .................................................119

MifSR TCS and $\alpha-K G$ dependent regulation of $P$. aeruginosa virulence.

123

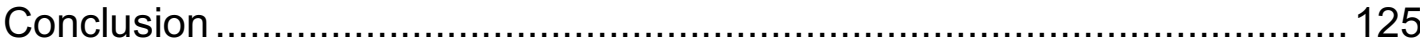

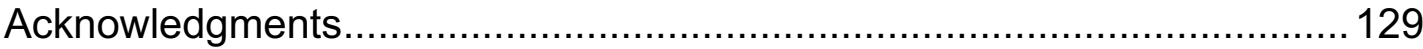

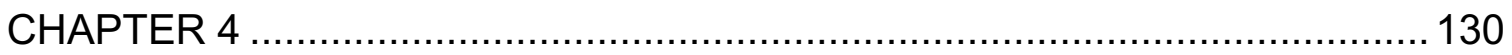

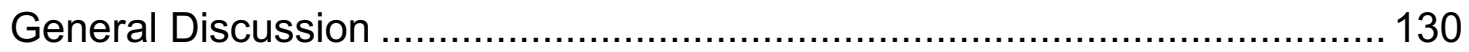

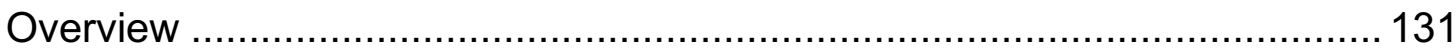

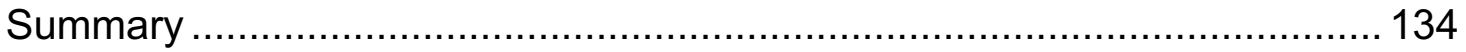

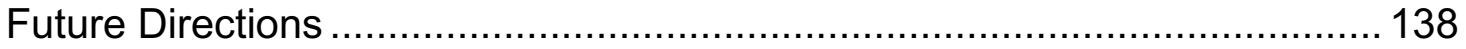

Determine the $\alpha-K G$ dependent MifR regulon ...................................139

Elucidate the role of RpoN $\left(\sigma^{54}\right)$ and TCS signaling in regulating

$P$. aeruginosa TCA cycle intermediate transport and pathogenesis.......147

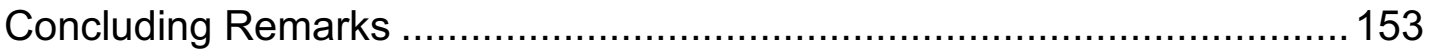

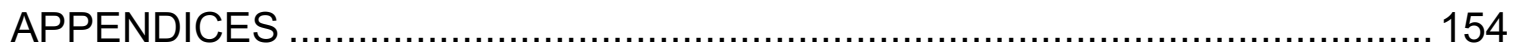

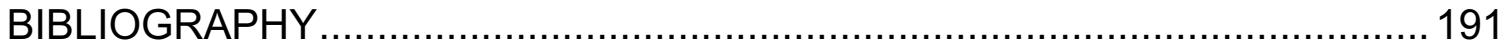

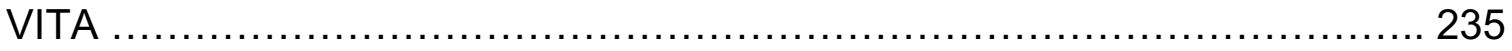




\section{LIST OF TABLES}

TABLE TITLE

PAGE

2.1 Strains and plasmids used in this study 24

$\begin{array}{lll}2.2 & \text { Primers used in this study }\end{array}$

2.3 Growth properties of mifSR mutants in presence of TCA 49 cycle intermediates

2.4 Growth profile analysis of the mifSR mutants in presence of 52 amino acids

2.5 Growth properties of PAO1 $\triangle r p o N$ and its derivatives in the $\quad 57$ presence of $\alpha-K G$ and LB

3.1 Strains and plasmids used in this study 90

$\begin{array}{lll}3.2 & \text { Primers used in this study } & 91\end{array}$

3.3 Growth Properties of the mifSR mutants and its derivatives 94 in the presence of $\alpha-K G$

A2.1 Comparison of extraction and analytical parameters 174 between this work and previous reports of biomarker detection from pathogens

A2.2 Bacterial strains used in this study 186

$\begin{array}{lll}\text { A2.3 Confusion matrix built upon blind study } & 187\end{array}$

$\begin{array}{lll}\text { A2.4 Mixture of bacterial cultures } & 188\end{array}$ 


\section{LIST OF FIGURES}

FIGURE TITLE

PAGE

1.1 Schematic representation of a prototypic two-component 5 system mediated signal transduction

2.1 Genome organization of the mifSR gene locus 37

$2.2 \quad$ Expression of poxB (PA5514) in mifSR mutants 38

2.3 mifS and mifR dependent phenotypes 41

2.4 Growth curve analysis in the presence of methionine 42

2.5 Phenotypic microarrays of PAO $\Delta$ mifS and PAO $\Delta$ mifR 43 mutants

2.6 Growth profile in the presence of varying concentrations of $\alpha-K G$

2.7 Rescue of $\alpha-K G-d e p e n d e n t$ growth phenotype of mifSR $\quad 45$

$2.8 \quad$ Tricarboxylic acid (TCA) cycle and its related reactions $\quad 47$

2.9 Growth curves in presence of $\alpha-K G$ in combination with 48 succinate and citrate

2.10 Quantification of $r p o N, a c n A, i d h, i c d, s u c A$, and $I p d 3$

2.11 Expression of PA5530 in response to $\alpha-K G$

$2.12 P$. aeruginosa MifR domain organization and sequence 56 alignment

2.13 silico analysis of mifS ( $\left.\mathrm{P}_{\text {mifS }}\right)$ and PA5530 ( $\left.\mathrm{P}_{\text {PA5530 }}\right)$
promoter sequences 


\section{LIST OF FIGURES}

FIGURE TITLE

PAGE

2.14 Proposed model for a-KG utilization in $P$. aeruginosa

59

3.1 Growth of mifSR mutants in the presence of $\alpha-K G$ and

94 pPcaT

3.2 pсa $T$ gene expression in response to $\alpha-K G$

97

3.3 Quantification of pcaT mRNA in mifSR mutants

98

3.4 Promoter analyses of $\alpha-K G$ permease encoding pcaT gene 99

$3.5 \quad \alpha-K G$ dependent regulation of biofilm formation by mifSR 104 mutants

3.6 Swimming and twitching motility of mifSR mutants in the presence of $\alpha-K G$

3.7 $\quad \alpha-K G$ specific swarming motility in mifSR mutants 106

3.8 Rhamnolipid production in the presence of $\alpha-K G \quad 110$

3.9 Pyocyanin production in mifSR mutants in the presence of 114 a-KG

3.10 Quantification of phenazine specific genes by qRT-PCR in the presence of

Pyoverdin production in mifSR mutants in the presence of $\alpha-K G$

3.12 Pyocyanin and Proverdine absorbance spectra

3.13 Cytotoxicity effect of $P$. aeruginosa PAO1 and mifSR

122 mutants on A549 lung fibroblast 


\section{LIST OF FIGURES}

FIGURE TITLE

PAGE

3.14 Cytotoxicity effect of $P$. aeruginosa PAO1 and mifSR

123 mutants on HeLA and J774-Eclone cells.

3.15 mifSR(pKgtP) virulence assays in the presence of $\alpha-K G$

Proposed model for a-KG-dependent MifS and MifR TCS

129 signal perception mechanism

4.1 Overview of $P$. aeruginosa virulence phenotypes differentially regulated by MifSR TCS in presence of $\alpha-K G$

4.2 Proposed model for $\alpha-K G$ dependent MifSR TCS signaling in $P$. aeruginosa

4.3 Effect of mifSR deletion on $P$. aeruginosa antibiotic resistance

4.4 Effect of mifSR deletion on $P$. aeruginosa virulence

4.5 Effect of mifSR deletion on extracellular protein concentration

4.6 Growth curve analysis in the presence of TCA cycle intermediates

4.7 P. aeruginosa RpoN and TCS mediated TCA cycle intermediate transport

A2.1 Capillary Microextraction of Volatiles (CMV) device

A2.2 The dynamic headspace sampling process using CMV device

A2.3 Chromatograph of headspace samples 


\section{LIST OF FIGURES}

FIGURE TITLE

PAGE

A2.4 Overlaid chromatograms for plates contain multiple bacteria 184 strains

A2.5 Non-mucoid ( $\left.\mathrm{Alg}^{-}\right)$and mucoid $\left(\mathrm{Alg}^{+}\right)$P. aeruginosa 185

The detection frequency of 1-undecene and 2-

185

A2.6 aminoacetophenone (2-AA) in mucoid and non-mucoid $P$. aeruginosa strains 


\section{LIST OF SYMBOLS}

$\begin{array}{ll}\text { SYMBOL } & \text { TERM / UNIT } \\ \alpha & \text { Alpha } \\ \beta & \text { Beta } \\ { }^{\circ} \mathrm{C} & \text { Degrees Celsius } \\ \Delta & \text { Delta (deletion) } \\ \% & \text { Percent } \\ \text { et al. } & \text { at alia } \\ \mu \mathrm{g} & \text { Microgram } \\ \mu \mathrm{l} & \text { Microliter } \\ \mu \mathrm{m} & \text { Micromolar }\end{array}$




\section{LIST OF ACRONYMS AND ABBREVIATIONS}

\begin{tabular}{|c|c|}
\hline ACRONYM/ & TERM \\
\hline \multicolumn{2}{|c|}{ ABBREVIATION } \\
\hline A549 & Human lung adenocarcinoma epithelial cell line \\
\hline$\alpha-K G$ & alpha-ketoglutarate \\
\hline ADP & Adenosine diphosphate \\
\hline ATP & Adenosine triphosphate \\
\hline Asp & Aspartate \\
\hline $\mathrm{bp}$ & Base pair \\
\hline $\mathrm{CaCl}_{2}$ & Calcium chloride \\
\hline cDNA & Complementary deoxyribonucleic acid \\
\hline CF & Cystic Fibrosis \\
\hline CR & Congo red \\
\hline CTAB & Cetyl trimethylammonium bromide \\
\hline DNA & Deoxyribonucleic acid \\
\hline E. coli & Escherichia coli \\
\hline E-test & Epsilometer test \\
\hline GC & Guanine-cytosine \\
\hline
\end{tabular}




\begin{tabular}{|c|c|}
\hline Glu & Glutamate \\
\hline Gln & Glutamine \\
\hline $\mathrm{His}$ & Histidine \\
\hline HK & Histidine kinase \\
\hline HTH & Helix-turn-helix \\
\hline J774 E-clone & Murine macrophage cell line \\
\hline $\mathrm{kb}$ & Kilobase \\
\hline $\mathrm{kDa}$ & Kilodalton \\
\hline LB & Luria Bertini \\
\hline MFS & Major facilitator superfamily \\
\hline $\mathrm{MgSO}_{4}$ & Magnesium sulfate \\
\hline $\mathrm{mg}$ & Milligram \\
\hline $\mathrm{ml}$ & Milliliter \\
\hline $\mathrm{mm}$ & Millimeter \\
\hline $\mathrm{mM}$ & Millimolar \\
\hline MIC & Minimum inhibitory concentration \\
\hline Min & Minutes \\
\hline $\mathrm{NaCl}$ & Sodium chloride \\
\hline
\end{tabular}




\begin{tabular}{|c|c|}
\hline $\mathrm{Na}_{2} \mathrm{HPO}_{4}$ & Sodium phosphate dibasic \\
\hline $\mathrm{NH}_{4} \mathrm{Cl}$ & Ammonium chloride \\
\hline $\mathrm{NADH}$ & Nicotiamide adenine dinucleotide \\
\hline OD & Optical Density \\
\hline $\mathrm{OM}$ & Outer membrane \\
\hline ORF & Open reading frame \\
\hline$P$. aeruginosa & Pseudomonas aeruginosa \\
\hline PCR & Polymerase chain reaction \\
\hline QS & Quorum Sensing \\
\hline RT-qPCR & Real time quantitative polymerase chain reaction \\
\hline $\mathrm{RR}$ & Response regulator \\
\hline RNA & Ribonucleic acid \\
\hline T3SS & Type III secretion system \\
\hline TCS & Two-component System \\
\hline$\mu g$ & Microgram \\
\hline$\mu l$ & Microliter \\
\hline$\mu \mathrm{m}$ & Micromolar \\
\hline
\end{tabular}




\section{CHAPTER 1}

\section{Introduction}

Parts of this chapter have been published:

Balasubramanian D., S.K. Murugapiran, E. Silva-Herzog, L. Schneper, X. Yang,

G. Tatke, G. Narasimhan, and K. Mathee. 2013.

In M, M. Babu. (ed.) Bacterial Gene Regulation and Transcriptional Networks.

Caister Academic Press, United Kingdom.

ISBN: 978-1-908230-14-0 


\section{Introduction}

"Adaptation is the key to survival", this is particularly true with bacterial cells as they are often exposed to an array of physiological and environmental changes during their lifecycle. The evolutionary success of bacteria is a testament to its intrinsic capability that helps them to keep pace with the frequently changing environmental conditions, by means of genetic and phenotypic changes. Although they are considered to be the simplest form of life, bacteria have evolved sophisticated molecular machinery that orchestrates multiple functions efficiently. Most bacteria, if not all, live in an extreme and/or dynamic environment, where an adaptive behavior mediated by quick response to ecological variations is a key to existence and proliferation. Bacterial cells achieved this by utilizing numerous signal transduction, information processing systems that incessantly monitor their surroundings for important changes in order to elicit an adaptive response.

Adaptation to environmental stimuli in bacteria is arbitrated predominantly through the expression of transcriptional regulators that include serine-threonine protein kinases (Kalantari et al., 2015), extra-cytoplasmic function (ECF) sigma factors (Mascher, 2013), and two-component signal transduction systems (TCSs) (Stock et al., 2000). These regulators function as cellular interpreters where they directly or with the help of other sensory units, first recognize a specific environmental stimuli at the cell surface and then transmit that extracellular information to the cytoplasm with the help of efficient signaling cascade (mostly phosphorylation and de-phosphorylation reactions) in order to stimulate an adaptive response, mainly by regulating expression of the target genes (Parkinson, 
1993, Goudreau \& Stock, 1998, Cashin et al., 2006). Amongst these signaling devices, TCS is the predominant signaling mechanism that empowers bacteria with an ability to efficiently sense, respond, and adapt to a wide variety of environments, stressors, and growth conditions (Stock et al., 2000).

\section{Two Component Regulatory Systems}

Two-component systems (TCS) are sophisticated signaling mechanisms marked by a highly modular design that have been adapted and integrated into a wide variety of cellular signaling circuits. They are one of the primary means by which bacteria sense and respond to variations in their environments, both intraand extracellular. The archetypical TCS is composed of a membrane integrated sensory histidine kinase $(\mathrm{HK})$ and a cytoplasmic response regulator (RR) (Figure 1.1). The HK contains a periplasmic $\mathrm{N}$-terminal domain that detects specific stimuli (sensing domain) and a C-terminal cytoplasmic transmitter domain that is comprised of a dimerization domain, a conserved histidine, and an ATP catalytic domain (Raghavan \& Groisman, 2010). HKs can have two or more transmembrane domains with little or no periplasmic domain while others are completely cytoplasmic. The cognate response regulator $(R R)$ contains a conserved receiver domain and a variable output domain (Stock et al., 2000). Upon receiving a signal, two HK monomers dimerize and cross-phosphorylate at the conserved histidine residue, the phosphate subsequently being transferred to an aspartate residue in the receiver domain of the cognate RR. The phosphotransfer is catalyzed by the receiver domain and results in a conformational change that activates the output domain, which often binds DNA and modulates gene expression or enzymatic 
activity (Stock et al., 2000, Mascher et al., 2006, Gooderham \& Hancock, 2009). Variations to this model occur in phosphorelays where a sensor kinase first transfers the phosphoryl group to a RR that has no output domain. The $P \sim R R$ then transfers the phosphoryl group to a histidine containing phosphotransfer protein (HPT), and this in turn serves as a phosphate donor to a terminal RR which has an output domain that mediates a cellular response. In other cases the sensor kinase and the RR lacking an output domain are fused into one protein (hybrid sensor kinase) (Gao \& Stock, 2010). Other variations include the TCS connectors, a group of proteins that modulate the phosphorylation state and activity of sensor HK and RR and establish regulatory links between otherwise independent signal transduction pathways (Mitrophanov \& Groisman, 2008).

In general, the mechanism of signal perception mediated by the archetypic bacterial two-component system in order to elicit an adaptive response can be summarized in four steps:

1) Signal detection by HK

2) ATP-dependent auto phosphorylation of the HK sensor at its C-terminal histidine residue (HK activation)

3) Transfer of phosphoryl group to the aspartate residue of the response regulator in the receiver domain (phosphortransfer and RR activation)

4) Regulation of target genes by the activated RR (response generation)

TCSs enable bacterial to effectively control their physiological and cellular functions by responding appropriately to a wide range of stimuli such as osmolarity, nutrient availability, temperature, $\mathrm{pH}$ and oxidative stress (Albright et al., 1989). 


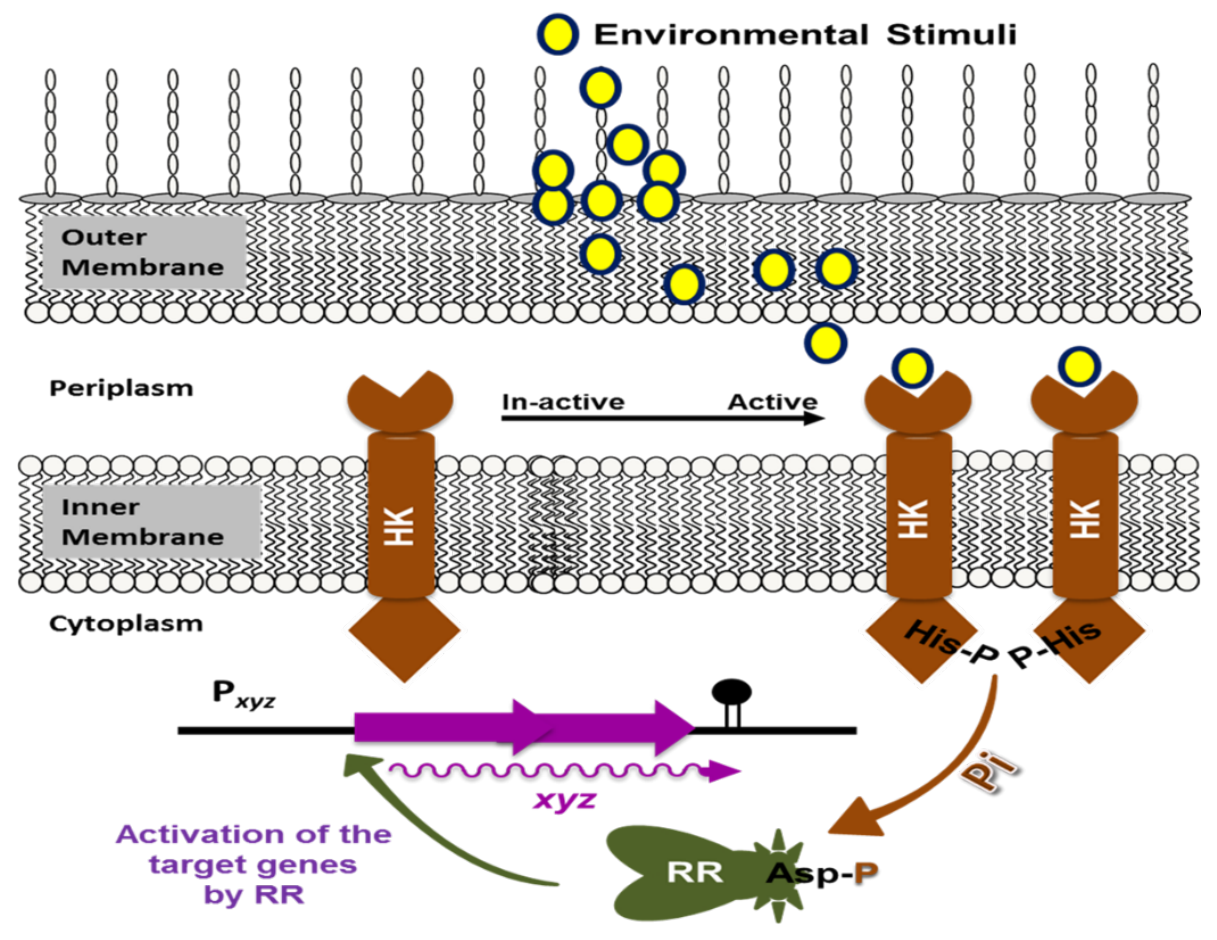

Figure 1.1: Schematic representation of a prototypic two-component system mediated signal transduction. TCS activation is arbitrated through a series of phosphotransfer reactions which typically involves phosphorylation and de-phosphorylation of the sensor histidine kinase (HK) and response regulator (RR). A specific environmental stimulus is detected by the $\mathrm{HK}$ through its sensor domain which brings about a conformational change resulting in two HK monomers to dimerize and auto cross-phosphorylate (ATP-dependent) at the conserved histidine residue located at its $\mathrm{C}$-terminal domain. The phosphorylated $\mathrm{HK}$ then serves as a phosphate donor for the RR receiver domain, resulting in phosphorylation of a conserved Aspartate residue. The phosphorylation of the aspartate residue subsequently results in the activation of the RR effector domain that mediates the cellular response, usually by differential expression of specific target genes. 


\section{$P$. aeruginosa and two-component systems}

Pseudomonas aeruginosa is a ubiquitous, Gram negative bacteria that exhibits extraordinary ability to colonize diverse ecological niches. Its metabolic versatility and adaptability has significantly contributed to its ubiquity (Ramos, 2004). Furthermore, $P$. aeruginosa causes opportunistic infection in humans, and is the primary pathogen responsible for pulmonary exacerbations and mortality in patients with cystic fibrosis (Lyczak et al., 2002). Importantly, it is one of the most recalcitrant nosocomial pathogen, responsible for $10 \%$ of all the hospital acquired infections (Sievert et al., 2013). Beyond high infection rate, $P$. aeruginosa has one of the highest case fatality rate amongst all Gram-negative infections (Aliaga et al., 2002). P. aeruginosa expresses an arsenal of multi-determinant virulence factors, both cell surface associated (flagella, pili, LPS) and secretory (proteases, exotoxins, phenazines, haemolysins), that play a crucial role in regulating its pathogenicity (Balasubramanian et al., 2013). In addition, a major impediment in treating $P$. aeruginosa infections is its extraordinary intrinsic and acquired resistance to several clinically critical antibiotics (Fraimow \& Nahra, 2013). These characteristics together ensures the success of $P$. aeruginosa as an intractable pathogen.

Furthermore, successful infection and disease progression depends significantly on the ability of bacterial pathogens, including $P$. aeruginosa, to adapt efficiently to the host environment. $P$. aeruginosa has evolved complex signal transduction mechanisms that aid in its effective adaptation by regulating and coordinating the temporal expression of various genes, in response to both inter- 
and intracellular signaling molecules and environmental cues (Hancock \& Speert, 2000, Rodrigue et al., 2000, Ventre et al., 2006, Gooderham \& Hancock, 2009, Williams \& Camara, 2009, Haussler, 2010, Jimenez et al., 2012). Amongst the gamut of signaling mechanisms identified in $P$. aeruginosa, TCS mediated signal perception play a predominant role in sensing diverse environmental stimuli and eliciting a pertinent and rapid adaptive response (Rodrigue et al., 2000, Stock et al., 2000).

$P$. aeruginosa, genome encodes one of the largest group of TCS proteins identified in any in any microorganism thus far analyzed (Barakat et al., 2009, Winsor et al., 2011). To date, 62 HK and 73 RR TCS proteins have been identified in $P$. aeruginosa prototypic strain PAO1 (Winsor et al., 2011). This provides the bacterium with a sophisticated capability to regulate diverse metabolic adaptations, virulence and antibiotic resistance processes that are hallmark of $P$. aeruginosa infections. What follows is a brief description of some prominent ones.

\section{PhoQ-PhoP}

PhoQ-PhoP is one of the most extensively studied TCS in the $\gamma$ proteobacterial species (Miller et al., 1989, Gooderham \& Hancock, 2009). ). It is composed of the inner membrane histidine sensor kinase (HK) PhoQ and the cytoplasmic response regulator (RR) PhoP (McPhee et al., 2006). This TCS was first identified in Salmonella enterica where it was reported to be involved in controlling the expression of non-specific acid phosphatase (Kier et al., 1979). Subsequently, a more profound role of the PhoPQ TCS was identified where it was 
involve in regulating several cellular activities including virulence mechanisms, cell cytotoxicity, motility, transport of small molecules, acid tolerance, antibacterial peptide resistance and bacterial surface remodeling (Groisman, 2001, Prost et al., 2008). The PhoQ-PhoP system has been identified as a major component of virulence in other Gram-negative bacteria including $P$. aeruginosa, Escherichia coli and S. enterica (Gooderham \& Hancock, 2009). The PhoPQ TCS is one of the first reported sensory-response mechanism to use extracellular cations as a primary signal (Gooderham \& Hancock, 2009).

In P.aeruginosa, PhoQ-PhoP TCS has been reported to modulate cellular adaptations to $\mathrm{Mg}^{2+}$-limiting environments (McPhee et al., 2006). It is now well established that sub-micromolar concentrations of $\mathrm{Mg}^{2+}$ induce P.aeruginosa PhoP-mediated transcription, and millimolar concentrations of $\mathrm{Mg}^{2+}$ are known to repress their expression (Gooderham et al., 2009). Furthermore, it is well established that the PhoPQ TCS respond to limiting concentrations of cations and regulate resistance to polymyxin $B$ and cationic antimicrobial peptides through the regulation of the arnBCDTEF-pmrE LPS modification operon (Macfarlane et al., 2000, McPhee et al., 2006) Activation of the genes in the arnBCADTEF operon results in the addition of 4-aminoarabinose to the Lipid A moiety of the lipopolysaccharide (LPS). The amalgamation of 4-aminoarabinose and Lipid A is responsible for inducing cytotoxicity in Gram-negative bacteria, because of a reduction of the net negative charge of the LPS. This limits LPS interaction with cationic peptides and cationic antibacterial agents such as polymixin B (McPhee et al., 2003, Moskowitz et al., 2004). PhoQ is also involved in swarming and 
twitching motility as well as in biofilm formation and it is required for virulence without affecting the T3SS or QS systems (Gooderham et al., 2009). It differs from the Salmonella PhoQ in that it is also involved in the regulation of transcription of genes outside the PhoP regulon, including $p m r A$, algU-mucABCD alginate production operon, Type II secretion proteins $\mathrm{HxcQ}$ and $\mathrm{XqhA}$, the quinolone signal biosynthetic genes $(p q s B C D)$, iron-scavenging-related genes and siderophore genes as well as energy-metabolism-related genes including nirO, nos $R$ and cytochrome o ubiquinol oxidase operon (Gooderham et al., 2009). Interestingly, $P$. aeruginosa isolates from cystic fibrosis patients have been shown to contain a lipid A structure equivalent to that of those formed by the activation of PhoPQ, and to generate an inflammatory response, suggesting a role of this TCS in eliciting an immune response. Furthermore, HK-PhoQ has been reported to crosstalk with other TCS regulatory proteins (PmrA/PmrB) to modulate several cellular responses (McPhee et al., 2003). McPhee and coworkers have shown that HKPhoQ is able to activate the RR-PmrA independently of HK-PmrB suggesting an interaction between these TCSs (McPhee et al., 2003). In addition, increased resistance to antibiotics including polymyxin $\mathrm{B}$, aminoglycosides and quinolones in phoQ mutants suggest crosstalk between PhoPQ and other TCS (Kwon \& Lu, 2006)

\section{GacS-GacA}

The sensor HK-GacS (PA0928) and the RR-GacA (PA2586) are members of a TCS that is present in a wide variety of Gram-negative bacteria (Heeb \& Haas, 2001). In $P$. aeruginosa, the GacSA TCS is central to the regulation of the 
expression of virulence factors, secondary metabolites, biofilm formation and QS (Kitten et al., 1998, Pessi et al., 2001) and to the switch between acute and chronic infections, as seen in mouse models of cystic fibrosis infection (Rahme et al., 2000, Coleman et al., 2003). GacS is a hybrid sensor HK that contains a HK domain, a RR domain and a histidine phosphotransfer (Hpt) domain (Goodman et al., 2004, Gooderham \& Hancock, 2009). The signal transduction pathway from GacA to individual virulence genes is complex. Phosphorylated GacA positively regulates the transcription of two small RNAs, $r s m Z$ and $r s m Y$, which block the negative regulator RNA-binding protein RsmA, allowing the expression of QS signals, swarming motility, rhamnolipid and lipid biosynthesis (Heeb \& Haas, 2001, Wolfgang et al., 2003, Brencic \& Lory, 2009). The GacSA TCS is also involved in antibiotic resistance to three different antibiotics: tobramycin, ciprofloxacin and tetracycline (Linares et al., 2006) apparently mediated also by RsmA/RsmZ. The mechanisms of resistance are poorly understood, and susceptibility to other antibiotics or antimicrobial peptides remains unaffected.

\section{RetS and LadS}

$P$. aeruginosa utilizes two orphan sensor kinases, RetS (regulator of exopolysaccharide and T3SS; PA4856) and LadS (lost adherence sensor, PA3974), to switch between acute and chronic infections and to regulate virulence mainly through the interaction with GacA-GacS (Goodman et al., 2004, Yahr \& Greenberg, 2004). RetS contains one HK domain and two tandem RR receiver domain (Goodman et al., 2004). LadS domain organization is similar to that of RetS, but it does not contain the second C-terminal RR domain (Ventre et al., 
2006). LadS regulates both early and late maturation stages of biofilm formation LadS and RetS are hybrid sensor kinases with a signaling domain consisting of a 7-transmembrane region and a periplasmic sensor domain that mediates homoand hetero-dimer formation (Goodman et al., 2004). RetS is required for T3SS activation, repression of biofilm formation, and colonization/dissemination in murine acute infection models (Goodman et al., 2004, Laskowski et al., 2004, Zolfaghar et al., 2005). Genes under RetS control are inversely regulated by two other sensor kinases: GacS and LadS, probably through the regulation of the small RNAs RsmZ and RsmA, as suggested by the demonstration that RetS can directly interact with GacS modulating the levels of RsmZ and RsmA (Ventre et al., 2006, Gooderham \& Hancock, 2009). LadS regulates biofilm formation probably through the regulation of the pel exopolysaccharide operon (Ventre et al., 2006). In a rat model with chronic pulmonary infection, LadS and RetS have been reported to regulate the expression of $\mathrm{Hcpl}$ secretion island I (required for chronic infection), thus are also thought to have an important role in chronic infection in CF by actively regulating Hcpl secretion island I (Potvin et al., 2003). LadS together with RetS and GacA/GacS/RsmZ forms a intricate and dynamic network that directs the expression of $P$. aeruginosa key virulence mechanisms resulting in acute infection (expression of Type III secretion) and chronic infections (biofilm formation) (Goodman et al., 2004, Yahr \& Wolfgang, 2006, Gooderham \& Hancock, 2009). However, the exact regulatory mechanism as to how LadS and RetS sensors bring about the cascade of signal transduction 
by modulating the expression of $r s m Z$ and $r s m Y$ transcription is not known, but it is possible that other response regulators may be involved (Laskowski \& Kazmierczak, 2006).

\section{NarL-NarX}

$P$. aeruginosa is often known to assume a biofilm mode of growth that is marked by a substantial metabolic alterations as a consequence of its genetic and phenotypic variations (Waite et al., 2006, Schreiber et al., 2007). The ability of $P$. aeruginosa to grow as biofilms can be largely attributed to its metabolic flexibility and adaptability that enables it to grow in a low nutrient and oxygen-limited environment, a characteristic of biofilms. In particular, anaerobic respiration by $P$. aeruginosa is considered to be a vital metabolic process during its growth as a biolfim in chronic pulmonary infections (Barraud et al., 2006, Palmer et al., 2007). Under anaerobic conditions, $P$. aeruginosa survives and supports its growth either by arginine fermentation or by denitrification (Palmer et al., 2005, Palmer et al., 2007). NarX and NarL TCS pair in $P$. aeruginosa is known to be involved in regulating $P$. aeruginosa denitrification pathways (Schreiber et al., 2007). Denitrification pathways play an important role in anaerobic respiration, where, in the absence of oxygen organism respire nitrate $\left(\mathrm{No}_{3}^{-}\right)$or nitrite $\left(\mathrm{No}_{2}^{-}\right)$. In P.aeruginosa, the Anr (PA1544), Dnr (PA0527) regulatory proteins in concert with NarL-NarX TCS control the expression of genes encoding proteins required for denitrification (Benkert et al., 2008). The NarX is a HK which contains a highly conserved nitrate recognition region. This region is termed as a P-box which recognizes environmental presence of nitrate to which NarX responds by 
autophosphorylating a conserved histidine residue (Stewart \& Bledsoe, 2008). Further, the transfer of high energy phosphate to the conserved aspartate residue of the response regulator NarL results in activation or repression of target operon (Stewart \& Bledsoe, 2008). Nitrate metabolism has been linked to motility, biofilm formation and virulence. NarX-HK is reported to be required for swarming motility, although the swarming phenotype has been displayed in a narL mutant as a result of overproduction of rhamnolipids (Van Alst et al., 2007). Also, narLX double mutants displayed reduced swimming motility in comparison to individual narL and narX mutants which exhibited normal motility, suggesting that the flagella dependent movement requires both the sensor kinase and the response regulator (Van Alst et al., 2007). Thus, the NarX-NarL TCS is involved in regulation several P. aeruginosa virulence related phenotypes including motility and biofilm formation.

\section{Conclusion:}

The versatility of two-component regulatory systems in sensing different signals, both extracellular and intracellular, make them an appropriate system for regulating expression of virulence and metabolism. $P$. aeruginosa genome encodes one of the largest groups of TCSs proteins (135), in sequenced bacteria (Winsor et al., 2011). Amongst these only few have been extensively studied and characterized in detail, including PhoP-PhoQ, GacA-GacS, RetS, LadS, and NarL-NarX, that are reported to be responsible for regulating $P$. aeruginosa key virulence determinants. Furthermore, in most of the cases, the environmental signals that influence TCS pathways remains obscure. However, there are a 
plethora of uncharacterized putative TCSs in P.aeruginosa. Taking into consideration the metabolic adaptability and the plethora of uncharacterized putative TCSs in P.aeruginosa, it is most likely that some of these putative systems may have important roles in virulence, antibiotic resistance and metabolism. Further research is required to characterize these role of these putative TCS by elucidate there activating signals, identifying their targets and also various regulatory networks into which TCS are integrated, since these systems can be appropriate drug targets for effective antimicrobial therapeutics. 


\section{CHAPTER 2}

Pseudomonas aeruginosa MifS-MifR two-component system is specific for a-ketoglutarate utilization

This Chapter has been published:

Gorakh Tatke, Hansi Kumari, Eugenia Silva-Herzog, Lourdes Ramirez, and Kalai Mathee 2015. PLoS ONE. 10(6):e0129629

DOI: 10.1371/journal.pone.0129629 


\section{Abstract}

Pseudomonas aeruginosa is a Gram-negative, metabolically versatile opportunistic pathogen that elaborates multitude of virulence factors, and is extraordinarily resistance to a gamut of clinically significant antibiotics. This ability, in part, is mediated by two-component regulatory systems (TCS) that play a crucial role in modulating virulence mechanisms and metabolism. MifS (PA5512) and MifR (PA5511) form one such TCS implicated in biofilm formation. MifS is a sensor kinase whereas MifR belongs to the NtrC superfamily of transcriptional regulators that interact with $\operatorname{RpoN}\left(\sigma^{54}\right)$. In this study we demonstrate that the mifS and mifR genes form a two-gene operon. The close proximity of mifSR operon to pox $B$ (PA5514) encoding a ß-lactamase hinted at the role of MifSR TCS in regulating antibiotic resistance. To better understand this TCS, clean in-frame deletions were made in $P$. aeruginosa PAO1 creating PAO $\Delta$ mifS, PAO $\Delta$ mifR and PAO $\Delta$ mifSR. The loss of mifSR had no effect on the antibiotic resistance profile. Phenotypic microarray (BioLOG) analyses of $\mathrm{PAO} \Delta$ mifS and $\mathrm{PAO} \Delta$ mifR revealed that these mutants were unable to utilize $C_{5}$-dicarboxylate $\alpha$-ketoglutarate $(\alpha-K G)$, a key tricarboxylic acid cycle intermediate. This finding was confirmed using growth analyses, and the defect can be rescued by mifR or mifSR expressed in trans. These mifSR mutants were able to utilize all the other TCA cycle intermediates (citrate, succinate, fumarate, oxaloacetate or malate) and sugars (glucose or sucrose) except $\alpha-K G$ as the sole carbon source. We confirmed that the mifSR mutants have functional dehydrogenase complex suggesting a possible defect in $\alpha-K G$ transport. The inability of the mutants to utilize $\alpha-K G$ was rescued by 
expressing PA5530, encoding $\mathrm{C}_{5}$-dicarboxylate transporter, under a regulatable promoter. In addition, we demonstrate that besides MifSR and PA5530, a-KG utilization requires functional RpoN. These data clearly suggests that $P$. aeruginosa MifSR TCS is involved in sensing $\alpha-K G$ and regulating its transport and subsequent metabolism. 


\section{Introduction}

Pseudomonas aeruginosa is a metabolically versatile, Gram-negative opportunistic pathogen that is well known for its extensive spatio-temporal distribution (Ramos, 2004). It is a dominant nosocomial pathogen capable of causing acute and chronic infections in immunocompromised and immunosuppressed patients (Ledizet et al., 2012, Turner et al., 2014). In particular, patients with AIDS, severe burn wounds, cystic fibrosis (Brencic et al.), chronic obstructive pulmonary disease (COPD), non-CF bronchiectasis and neutropenia are predisposed to $P$. aeruginosa infections (Lyczak et al., 2000, Manfredi et al., 2000, Ramos, 2004, Furukawa et al., 2006, Valderrey et al., 2010). P. aeruginosa chronic pulmonary infections are characterized by intensive bronchial neutrophilic inflammation resulting in respiratory failure (Emerson et al., 2002, CohenCymberknoh et al., 2013), a major cause of fatality in CF patients (Lyczak et al., 2002). Moreover, $P$. aeruginosa is associated with keratitis (Dart \& Seal, 1988) and chronic suppurative otitis media (Yeo et al., 2007) leading to visual impairment and deafness (Prevatt et al., 2004, Sun et al., 2010). P. aeruginosa possess numerous virulence factors, both cell-surface associated and secretory, which significantly contribute to its pathogenesis (Balasubramanian et al., 2013). Effective treatment of $P$. aeruginosa infections is impeded by its extraordinary intrinsic and acquired resistance to numerous clinically important antibiotics (Fraimow \& Nahra, 2013). Thus, antibiotic resistance and expression of multi-determinant virulence factors are two critical hallmarks in $P$. aeruginosa infections that make it an intimidating pathogen. 
Successful infection and disease progression depends significantly on the ability of any pathogen to effectively utilize available nutrients that are essential for its growth and survival. P. aeruginosa is renowned for its extraordinary ability to utilize wide range of organic compounds such as carbohydrates, amino acids, fatty acids, mono- and polyalcohols, di- and tri-carboxylic acids as sources of carbon, nitrogen and energy (Ramos, 2004). However, unlike other bacteria where glucose is the preferred carbon source (Loomis \& Magasanik, 1967, Stulke \& Hillen, 2000), $P$. aeruginosa preferentially utilizes tricarboxylic acid (TCA) cycle intermediates (Wolff et al., 1991, Collier et al., 1996), specifically, $C_{4}$-dicarboxylates of the TCA cycle such as malate, fumarate and succinate (Liu, 1952, Wolff et al., 1991, Collier et al., 1996).

The TCA cycle is an amphibolic pathway that serves two main purposes: energy-generation in aerobic organisms (catabolism), and the generation of intermediates to serve as biosynthetic precursors for fatty acid, amino acid and carbohydrate synthesis (anabolism) (Owen et al., 2002). The metabolic intermediates of the TCA cycle consist of a group of organic anions that include $\mathrm{C}_{4}$-dicarboxylates (succinate, fumarate, malate and oxaloacetate), $\mathrm{C}_{5}$ dicarboxylates (alpha-ketoglutarate $(\alpha-K G)$ ) and $\mathrm{C}_{6}$-tricarboxylates (citrate, isocitrate) (Krebs, 1940, Frohman et al., 1951). However, the role of TCA cycle intermediates is not restricted to energy metabolism or to serve as biosynthetic precursors. In the recent years, TCA cycle intermediates, in-particular, succinate and/or $\alpha-K G$ have gained significant importance as biological signaling molecules 
in variety of organisms including, bacteria (Ninfa \& Jiang, 2005), animals (He et al., 2004) and plants (Feria Bourrellier et al., 2010).

Sensing the available nutrients is a prerequisite for mobilizing the uptake systems. Bacterial two-component systems (TCSs), involving a membrane-bound histidine sensor kinase (HK) and a cytoplasmic response regulator (RR) play an integral part in bacteria's ability to sense physiological cues. In response to stimuli, the sensor autophosporylates at a conserved histidine residue at the C-terminus, and subsequently the phosphate is transferred to an aspartate residue at the $\mathrm{N}$ terminus of the RR (Stock et al., 1989, Hoch \& Silhavy, 1995, Asai et al., 2000). TCSs in Bacillus subtilis, Corynebacterium glutamicum, Escherichia coli, Klebsiella pneumoniae, Rhizobium meliloti and Rhizobium leguminosarum have been shown to regulate extracellular $\mathrm{C}_{4}$-dicarboxylates and tricarboxylates transport (Jiang et al., 1989, Bott et al., 1995, Reid \& Poole, 1998, Golby et al., 1999, Asai et al., 2000, Yamamoto et al., 2000, Brocker et al., 2009). Of these, DctB-DctD in $R$. meliloti is an extensively studied TCS, which in coordination with sigma factor $\operatorname{RpoN}\left(\sigma^{54}\right)$ regulates the extracellular transport of $\mathrm{C}_{4}$-dicarboxylates succinate, fumarate and malate (Ronson et al., 1987, Watson, 1990).

Three TCS protein pairs in $P$. aeruginosa namely, PA5165/PA5166 (DctB/DctD), PA5512/PA5511 (MifS/MifR) and PA1336/PA1335 have been identified to be homologous to the Rhizobium $\mathrm{C}_{4}$-dicarboxylate transport regulatory DctB/DctD (Valentini et al., 2011). Amongst the three, very little is known of PA1336/PA1335. The PA5165/PA5166 (DctB/DctD) TCS has been demonstrated 
to regulate the transport of $\mathrm{C}_{4}$-dicarboxylates, succinate, fumarate and malate in coordination with the sigma factor RpoN $\left(\sigma^{54}\right)$ (Valentini et al., 2011). The SK MifS (65.3 $\mathrm{kDa})$ and RR MifR ( $49.6 \mathrm{kDa})$ share $51 \%$ and $69 \%$ sequence identity to the R. meliloti DctB and DctD, respectively (Stover et al., 2000). The RR MifR is involved in regulating the maturation stage of $P$. aeruginosa biofilm formation as mifR deficient mutants fail to form microcolonies (Petrova \& Sauer, 2009). Later studies reported the interdependence of pyruvate fermentation and functional MifR in supporting microcolony formation (Petrova et al., 2012). However, the mechanism by which MifR is activated in this process remains obscure and no relation with HK MifS has been established. Using clean in-frame deletion mutants of the mifS, mifR and mifSR genes we show that MifSR TCS regulates $P$. aeruginosa $\alpha-K G$ transport and requires functional RpoN.

\section{Materials \& Methods}

\section{Strains, media and growth conditions}

P. aeruginosa wild-type PAO1 (Stover et al., 2000) and its derivatives $\mathrm{PAO} \Delta$ mifS, PAO $\Delta$ mifR, PAO $\Delta$ mifSR and $\mathrm{PAO} \Delta r p o N$ or Escherichia coli strain DH5 $\alpha$ were used in this study (Table 2.1). Saccharomyces cerevisiae strain InvSC1 (Invitrogen, Life Technologies, Carlsbard, CA, USA) was used for in vivo homologous recombination (Bascom-Slack \& Dawson, 1998). Briefly, all bacterial cultures were grown in Luria Bertani (LB) broth (5 g tryptone, $10 \mathrm{~g}$ sodium chloride, and $5 \mathrm{~g}$ yeast extract per liter) or agar (LB broth with 1.5\% agar) (Difco, NJ, USA) or M9 minimal Media $\left(64 \mathrm{~g} \mathrm{Na}_{2} \mathrm{HPO}_{4}-7 \mathrm{H}_{2} \mathrm{O}, 15 \mathrm{~g} \mathrm{KH}_{2} \mathrm{PO}_{4}, 2.5 \mathrm{~g} \mathrm{NaCl}, 5.0 \mathrm{~g} \mathrm{NH}_{4} \mathrm{Cl}\right.$, $20 \mathrm{mM} \mathrm{MgSO}_{4}, 1 \mathrm{mM} \mathrm{CaCl}_{2}$ per liter) (Sambrook \& Russell, 2001) at $37^{\circ} \mathrm{C}$, unless 
specified otherwise. Yeast extract-peptone-dextrose media (YEPD: $20 \mathrm{~g}$ Bacto Peptone, $10 \mathrm{~g}$ yeast extract, $20 \mathrm{~g}$ dextrose per liter) was routinely used to culture S. cerevisiae and synthetic define agar-uracil media was used as selection media for pMQ30 yeast transformants (Shanks et al., 2006). P. aeruginosa competent cells were prepared as previously described (Choi et al., 2006). For growth curve and complementation studies M9 minimal media supplemented with glucose, sucrose or TCA cycle intermediates including citrate, $\alpha-K G$, succinate, fumarate, malate or oxaloacetate were used as a sole carbon source at $30 \mathrm{mM}$ each unless specified otherwise. Motility assays were performed in LB media (Difco, NJ, USA). For pyocyanin and proverdine production strains were cultivated in King's A medium (Difco, NJ, USA) and King's B medium (King et al., 1954). Cation-adjusted Mueller Hinton broth and agar (Difco, NJ, USA) was used in MIC assays. For plasmid maintenance, antibiotics were added to growth media when appropriate, at the specified concentrations: E. coli: ampicillin (Ap) $100 \mu \mathrm{g} / \mathrm{ml}$, gentamycin (Siegmund \& Wagner) $15 \mu \mathrm{g} / \mathrm{ml}$, kanamycin (Km) $20 \mu \mathrm{g} / \mathrm{ml}, P$. aeruginosa: Gm 75 $\mu \mathrm{g} / \mathrm{ml}$.

\section{Genetic manipulations}

Genetic manipulations were carried out using standard techniques (Sambrook \& Russell, 2001). Primers were synthesized by Integrated DNA Technologies, Inc. (Coralville, IA, USA) and are listed in Table 2.2. Plasmid DNA isolation was carried out using PureLink Hipure Plasmid Miniprep Kit (Invitrogen, Life Technologies, Carlsbard, CA, USA) and agarose gel fragments were purified using Wizard SV Gel and PCR Clean-Up System (Promega, Madison, WI, USA). 
RNA and cDNA was made using RNeasy Mini Kit (Qiagen Inc. Venio, Limburg, Netherlands) and SuperScript III First-Strand Synthesis System (Invitrogen, Life Technologies, Carlsbard, CA, USA). Restriction endonucleases were from New England Biolabs (Ipswich, MA, USA) and DNA sequencing was carried out at Florida International University (FIU) DNA core and at GENEWIZ Inc (South Plainfield, NJ, USA). All other chemicals were purchased from SIGMA-ALDRICH (St. Louis, MO, USA), AMRESCO (Solon, OH, USA) and Fisher Scientific (Waltham, MA, USA).

Table 2.1: Strains and plasmids used in this study.

\begin{tabular}{|c|c|c|c|}
\hline Strain ID & $\begin{array}{c}\text { Strain/Plasmid } \\
\text { Background }\end{array}$ & Relevant characteristics & Source \\
\hline \multicolumn{4}{|c|}{ Escherichia coli } \\
\hline $\mathrm{DH} 5 \alpha$ & E. coli & $\begin{array}{l}\mathrm{F}^{-} \text {Ф80lacZDM15D } \\
\text { (lacZYA-argF)U169 deoR } \\
\text { recA1 endA1 hsdR17 (rk } \\
\mathrm{mk}^{+} \text {) phoA supE44 } \lambda^{-} \text {thi-1 } \\
\text { gyrA96 relA1 }\end{array}$ & $\begin{array}{l}\text { New England } \\
\text { Biolabs }\end{array}$ \\
\hline \multicolumn{4}{|c|}{ Saccharomyces cerevisiae } \\
\hline INVSc1 & S. cerevisiae & $\begin{array}{l}\text { MATa his3D1 leu2 trp1- } \\
289 \text { ura3-52 }\end{array}$ & Invitrogen \\
\hline \multicolumn{4}{|c|}{ Pseudomonas aeruginosa } \\
\hline PAO1 & & Prototypic wild type & $\begin{array}{c}\text { (Stover et al., } \\
2000)\end{array}$ \\
\hline PKM900 & PAO1 & $\Delta m i f S(P A 5512)$ & $\begin{array}{l}\text { PAO } \triangle \text { mifS; This } \\
\text { study }\end{array}$ \\
\hline PKM901 & PAO1 & $\Delta \operatorname{mifR}(P A 5511)$ & $\begin{array}{l}\text { PAO } \triangle \text { mifR; This } \\
\text { study }\end{array}$ \\
\hline PKM902 & PAO1 & $\triangle$ mifSR (PA5511-PA5512) & $\begin{array}{l}\mathrm{PAO} \triangle \text { mifSR;Thi } \\
\text { s study }\end{array}$ \\
\hline $\mathrm{PAO} \Delta r p o N$ & PAO1 & $\Delta r p o N(P A 4462)$ & $\begin{array}{l}\text { (Heurlier et al., } \\
\text { 2003) }\end{array}$ \\
\hline $\begin{array}{l}\text { PAO1 } 1 \text { rpoN } \\
\because: r p o N\end{array}$ & PAO1 & $\begin{array}{l}\Delta r p o N \text { att Tn7::rpoN_} \\
\text { aacC1 }\end{array}$ & $\begin{array}{l}\text { (Heurlier et al., } \\
\text { 2003) }\end{array}$ \\
\hline
\end{tabular}




\begin{tabular}{|c|c|c|c|}
\hline Plasmids & & & \\
\hline $\begin{array}{l}\text { pCR2.1 } \\
\text { TOPO }\end{array}$ & & $\begin{array}{l}\mathrm{Ap}^{\mathrm{R}}, \mathrm{Km}^{\mathrm{R}} ; \text { colE1 f1 ori } \\
\text { lacZa }\end{array}$ & Invitrogen \\
\hline pRK600 & & $\mathrm{Cm}^{\mathrm{R}}$; colE1 tra ${ }^{+} \mathrm{RK} 2 \mathrm{mob}^{+}$ & $\begin{array}{c}\text { (Kessler et al., } \\
\text { 1992) }\end{array}$ \\
\hline pRK2013 & & $\mathrm{Km}^{\mathrm{R}}$; colE1 tra $^{+} \mathrm{RK} 2 \mathrm{mob}^{+}$ & $\begin{array}{c}\text { (Figurski \& } \\
\text { Helinski 1979) }\end{array}$ \\
\hline pEXG2 & & $\begin{array}{l}\mathrm{Gm}^{\mathrm{R}} ; \text { colE1, oriT } \mathrm{mob}^{+} \\
\mathrm{sacB}^{+}\end{array}$ & $\begin{array}{l}\text { (Rietsch et al., } \\
\text { 2005) }\end{array}$ \\
\hline pMQ30 & & $\mathrm{Gm}^{\mathrm{R}} ;$ colE1, oriT & $\begin{array}{c}\text { (Shanks et al., } \\
\text { 2006) }\end{array}$ \\
\hline pPSV37 & & $\begin{array}{l}\mathrm{Gm}^{\mathrm{R}} ; \text { colE1 orit lacl } \\
\mathrm{P}_{\text {lacuv5 }}\end{array}$ & $\begin{array}{c}\text { (Lee et al. } \\
\text { 2010) }\end{array}$ \\
\hline pGDT001 & pCR2.1 TOPO & $\begin{array}{l}\text { Ap }{ }^{R} \text {; A } ~ 1.7-k b \text { Nhel-Xbal } \\
\text { fragment containing mifS } \\
\text { ORF (PA5512) amplified } \\
\text { from PAO1 genome using } \\
\text { HK_mifSF1 and } \\
\text { HK_mifSR1 primers and } \\
\text { cloned into pCR } 2.1 \text { TOPO }\end{array}$ & This study \\
\hline pGDT002 & pCR2.1 TOPO & $\begin{array}{l}\mathrm{Ap}^{\mathrm{R}} ; \mathrm{A} \sim 1.3-\mathrm{kb} \text { Nhel-Sacl } \\
\text { fragment containing mifR } \\
\text { (PA5511) ORF amplified } \\
\text { from PAO1 genome using } \\
\text { GDT_mifRF1 and } \\
\text { GDT_mifRR1 primers and } \\
\text { cloned into pCR } 2.1 \text { TOPO }\end{array}$ & This study \\
\hline pGDT003 & pPSV37 & $\begin{array}{l}\mathrm{Gm}^{\mathrm{R}} \text {; The mifS ORF } \\
\text { subcloned from pGDT001 } \\
\text { as an Nhel-Xbal fragment } \\
\text { into pPSV37 }\end{array}$ & $\begin{array}{l}\text { pMifS } \\
\text { This study }\end{array}$ \\
\hline pGDT004 & pPSV37 & $\begin{array}{l}\mathrm{Gm}^{\mathrm{R}} \text {; The mifR ORF } \\
\text { subcloned from pGDT002 } \\
\text { as an Nhel-Sacl fragment } \\
\text { into pPSV37 }\end{array}$ & $\begin{array}{l}\text { pMifR } \\
\text { This study }\end{array}$ \\
\hline pGDT005 & pPSV37 & $\begin{array}{l}\text { Gm }{ }^{\mathrm{R}} \text {; A } ~ 3.0-\mathrm{kb} \text { Nhel-Sacl } \\
\text { fragment containing mifSR } \\
\text { (PA5511-PA5512) ORFs } \\
\text { amplified from PAO1 } \\
\text { genome using HK_mifSF1 } \\
\text { and GDT_mifRR1 primers } \\
\text { and cloned directly into } \\
\text { Nhel-Sacl-cut in pPSV37 }\end{array}$ & $\begin{array}{l}\text { pMifSR } \\
\text { This study }\end{array}$ \\
\hline pGDT006 & pPSV37 & $\begin{array}{l}\mathrm{Gm}^{\mathrm{R}} ; \mathrm{A} \sim 1.3-\mathrm{kb} \text { Nhel-Sacl } \\
\text { fragmentcontaining }\end{array}$ & $\begin{array}{l}\text { pPA5530 This } \\
\text { study }\end{array}$ \\
\hline
\end{tabular}


PA5530 ORF amplified from $\mathrm{PAO} 1$ genome using GDT_PA5530F1 and GDT_PA5530R1 primers and cloned directly into Nhel-Sacl-cut in pPSV37 
Table 2.2. Primers used in this study.

\begin{tabular}{|c|c|}
\hline Primer Name & Sequence \\
\hline HKmifSUF & $\begin{array}{l}\text { 5'- } \\
\text { GGAATTGTGAGCGGATAACAATTTCACACAGGAA } \\
\text { ACAGCTTCAGCTCGACTCCGCCGTCG-3' }\end{array}$ \\
\hline HKmifSUR & $\begin{array}{l}\text { 5'- } \\
\text { GACGAAGATCACCTGGTCGCCTAGTTAGCTAGCA } \\
\text { TCGGCGGATCGAAACGGC-3' }\end{array}$ \\
\hline HKmifSDF & $\begin{array}{l}\text { 5'- } \\
\text { GCCGTTTCGATCCGCCGATGCTAGCTAACTAGGC } \\
\text { GACCAGGTGATCTTCGTC-3' }\end{array}$ \\
\hline HKmifSDR & $\begin{array}{l}\text { 5'- } \\
\text { CCAGGCAAATTCTGTTTTATCAGACCGCTTCTGCG } \\
\text { TTCTGATACCGCTCTCATGACCGAA-3' }\end{array}$ \\
\hline mifRUF1 & 5'-TTTGAATTCGCCTGGTCGAGCAGCGCA-3' \\
\hline mifRUR1 & 5'-TTTGCTAGCTCGCTCATGTCG-3' \\
\hline mifRDF1 & 5'-TTTAAGCTTCTCGGCTTCGACGCCCAT \\
\hline mifRDR1 & 5'-TTTGCTAGCTCGCGAGGCGTC-3' \\
\hline mifSRUF1 & $\begin{array}{l}\text { 5'- } \\
\text { GGAATTGTGAGCGGATAACAATTTCACACAGGAA } \\
\text { ACAGCTGCGAGCACCAGCGCGCCACT-3' }\end{array}$ \\
\hline mifSRUR1 & $\begin{array}{l}\text { 5'- } \\
\text { TCTCTGACGCCTCGCGAGGGCTGCTCTAGTTAGC } \\
\text { TAGCATCGGCGGATCGAAACGGCGGC-3' }\end{array}$ \\
\hline mifSRDF1 & $\begin{array}{l}\text { 5'- } \\
\text { GCCGTTTCGATCCGCCGATGCTAGCTAACTAGAG } \\
\text { CAGCCCTCGCGAGGCGTCAGAGA-3' }\end{array}$ \\
\hline mifSRDR1 & $\begin{array}{l}\text { 5'- } \\
\text { CCAGGCAAATTCTGTTTTATCAGACCGCTTCTGCG } \\
\text { TTCTGATTACGTGTTCAGCGCGCTG-3' }\end{array}$ \\
\hline HK_mifSF1 & $\begin{array}{l}\text { 5'- } \\
\text { GCTAGCAGAAGGAGATATACCATGTCCTTGTCCC } \\
\text { GTCCGCTG-3' }\end{array}$ \\
\hline HK_mifSR1 & 5'-TTTICIAGATCATGTCGTTACGCTCGTGTC-3' \\
\hline GDT_mifRF1 & $\begin{array}{l}\text { 5'- } \\
\text { GCTAGCAGAAGGAGATATACCATGAGCGACCAG } \\
\text { GTGATCTTCGTCGAC-3' }\end{array}$ \\
\hline GDT_mifRR1 & 5'-TTTGAGCTCTGCTTCAGGCCGGCTCTTCGC-3' \\
\hline GDT_PA5530F1 & $\begin{array}{l}\text { 5'- } \\
\text { GCIAGCAGAAGGAGATATACCATGGAAAGCGCCA }\end{array}$ \\
\hline
\end{tabular}




\begin{tabular}{ll}
\hline GDT_PA5530R1 & 5'- \\
& TTTGAGCTCTCAATCGGTCGTGATCTTCGAGTGC- \\
'' & \\
GDT_cotransF1 & 5'-GGTGTTCAGCCTGATCCTGCCGG-3' \\
GDT_cotransR1 & 5'-CCGCTTCGCGGATCGTCGCTTC-3' \\
GDT_cotransF2 & 5'-GGATCGTCCACGAACTCGGCGGC-3' \\
GDT_cotransR2 & 5'-CAGGCGCACCTCGAAGCCGGAC-3' \\
GDT_p37_SeqF & 5'-GACCCGTTTAGAGGCCCCAA-3' \\
GDT_p37_SeqR & 5'-CGTGCTTTACACTTTATGCTTCCGG-3' \\
mifR_seqF & 5'-TGGTGCTGGAGAACCGGC-3' \\
mifR_seqR & 5'-GCAGTTCAGCGCCACGAAC-3' \\
mifS_SeqF & 5'-ATCTGGAACGGCAGTGGAACC-3' \\
mifS_SeqF2 & 5'-ATCGACGGCGAGTTGCAGCA-3' \\
PA5530_seqF & 5'-TCGCGGCATGGAAGAGAC-3' \\
PA5530_seqR & 5'-CATGCCGCGACGCAG -3' \\
DBS_qRT_clpXF & 5'- TGCGATTACGATGTGGAGA -3' \\
DBS_qRT_clpXR & 5'-CCCTCGATGAGCTTCAGCA -3' \\
GDT_qRT_PA5530F & 5'-CGCAACGCATCAAGTCGAT-3' \\
GDT_qRT_PA5530R & 5'-AGTCGTACCACTCGACCAGGTT-3' \\
qRT_rpoNF & 5'-AAATGCGAAAAAGCCATTGAG-3' \\
qRT_rpoNR & 5'-CCCTGTGCCTCCAGTAAACC-3' \\
qRT_icdF & 5'-GCGACCGGTGACAAAATCAC-3' \\
qRT_icdR & 5'-GGGTTCTTCGGTACGCTCAA-3' \\
qRT_idhF & 5'-GGCGATGATCCGCAACTC-3' \\
qRT_idhR & 5'-GCATTACCGCCTTGGTGTCT-3' \\
qRT_sucAF & 5'-CTGCAGCCAGCATCACATG-3' \\
qRT_sucAR & 5'-CGAGATTGAGGCCCTTCTTG-3' \\
qRT_Ipd3F & 5'-CATGCGGCGGAGATGAAC-3' \\
qRT_Ipd3R & 5'-ACTTCCGGCTGGGTGTAGATG-3' \\
\hline &
\end{tabular}

qRT in the primer name indicates that the primer was designed for qPCR. Broken and continuous lines below the primer sequence indicate ribosome binding and restriction sites respectively. 


\section{Construction of $P$. aeruginosa $\Delta$ mifR mutant}

An unmarked mifR clean in-frame deletion mutant of $P$. aeruginosa was generated by gene splicing (Horton et al., 1990). Upstream and downstream flanking regions of mifR were amplified by PCR (GC Rich PCR System, Roche, Indianapolis, IN, USA), using primers listed in Table 2.2. A 754-bp P1 and a 720bp P2 were amplified using upstream primers mifRUF1-EcoRI and mifRUR1-Nhel and the downstream primers mifRDF1-Nhel and mifRDR1-HindllI (Table 2.2), respectively from PAO1 genomic DNA. After sequencing to ensure fidelity, P1 and P2 were spliced together to obtain a 1474-bp deletion fragment with a deletion of mifR containing stop codons at its junction (inserted as part of Nhel site in the primer). This was then sequenced and subcloned into a $P$. aeruginosa nonreplicative plasmid pEXG2 (Rietsch et al., 2005) as a EcoRI-HindIII fragment and moved into the wild-type PAO1 strain by allelic replacement (Schweizer \& Hoang, 1995) using pRK600 and pRK2013 as the helper plasmids (Figurski \& Helinski, 1979, Kessler et al., 1992) . Clones were screened for $\mathrm{Gm}$ sensitivity $\left(75 \mu \mathrm{g} \mathrm{ml}^{-1}\right)$ and sucrose resistance ( $8 \%$ sucrose) corresponding to a double cross-over recombination event and replacement of the target gene with the deletion product. The presence of the deletion in PAOAmifR (PKM901) was confirmed by PCR amplification and sequencing of the deletion product (data not shown).

\section{Construction of $P$. aeruginosa $\Delta m i f S$ and $\Delta m i f S R$ mutants}

The unmarked mifS and mifSR deletion in PAO1 was generated by using the yeast system of double-stranded gap repair and homologous recombination (Shanks et al., 2009). Briefly, the mifS and mifSR upstream and downstream 
flanking regions were amplified by PCR using primers listed in Table 2.2. To create a mifSR deletion, an upstream 933-bp P1 and a downstream 1115-bp P2 were amplified using primer pairs mifSRUF1-mifSRDF1 and mifSRUR1-mifSRDR1, respectively. Similarly, to create mifS deletion, an upstream 703-bp P1 and a downstream 653-bp were amplified using primer pairs HKmifSUF-HKmifSDF and HKmifSUR-HKmifSDR, respectively. HKmifSUF and mifSRUF1 primers had stretches of homologous

DNA, 5'GGAATTGTGAGCGGATAACAATTTCACACAGGAAACAGCT-3' and 5'CCAGGCAAATTCTGTTTTATCAGACCGCTTCTGCGTTCTGAT-3', respectively, to target recombination of the amplicons with pMQ30 vector. These primer pairs also had complementing sequences at the 3 ' end to facilitate joining to create the P3 fragment, as well as stop codons (CTAGTTAGCTAG) to prevent any run off translation. The pMQ30 vector has double selection markers URA3 for yeast and gentamycin for E. coli (Shanks et al., 2006). Yeast cells were transformed with the P1, P2 and linearized pMQ30 (BamHI digested) using standard protocols and (Shanks et al., 2009) colonies were selected on sucrose-uracil plates.

The yeast colonies were checked for the presence of P3 constructs for mifS and mifSR deletions by amplification using upstream forward (mifSRUF1 and HKmifSUF, respectively) and downstream reverse (mifSRDR1 and HKmifSDR, respectively) primers. Yeast DNA was isolated from the positive colonies as described earlier (Shanks et al., 2009). E. coli was transformed with the recombinant $\mathrm{pMQ} 30$ plasmids containing $\mathrm{P} 3 \mathrm{~s}$ and screened for gentamycin resistance. The amplified P3s from the recombinant plasmids were sequenced to 
ensure fidelity. The constructs were then moved into PAO1 strain using tri-parental mating and screened for single and double crossovers using counter selection with sucrose and gentamycin as described earlier (Balasubramanian et al., 2012, Kumari et al., 2014). The presence of the gene deletions in all the mutants were confirmed using standard molecular methods (PCR and DNA sequencing of the locus). These strains are henceforth referred to as PAOAmifS (PKM900) and $\mathrm{PAO} \Delta$ mifSR (PKM902).

\section{Construction of complementing plasmids}

DNA fragments from $P$. aeruginosa PAO1 with mifS $(\sim 1.77 \mathrm{~kb})$ and mifR $(\sim 1.35 \mathrm{~kb})$ were PCR amplified using primer pairs HK_mifSF1-HK_mifSF1R1, GDT_mifRF1-GDT_mifRR1, respectively. In order to ensure expression of the genes, the primers are designed such that the ORF will juxtapose against a strong ribosome binding site (Lehninger et al., 2013). The PCR amplified products were cloned into pCR2.1 TOPO (Invitrogen, Life Technologies, Carlsbard, CA, USA) using manufacturers protocol to generate plasmids pGDT001 and pGDT002, respectively. The fidelity of the PCR amplified product was confirmed by sequencing. The fragments carrying mifS and mifR were moved into a broad host range pPSV37-Gm plasmid (Lee et al., 2010) as a Nhel-Sacl fragments, downstream of an inducible $\mathrm{P}_{\text {lacUv5 }}$ promoter to generate plasmids PGDT003 and pGDT004, respectively. Henceforth, these plasmids are referred to as pMifS and pMifR.

DNA fragments from PAO1 with mifSR $(\sim 3.12 \mathrm{~kb})$ and PA5530 $(\sim 1.3 \mathrm{~kb})$ were PCR amplified using primer pairs HK_mifSF1-GDT_mifRR1 and 
GDT_PA5530F1-GDT_PA5530R1 (Table 2.2), respectively. The PCR amplified products were cloned directly into pPSV37-Gm plasmid as Nhel-Sacl fragments, downstream of an inducible $\mathrm{P}_{\text {lacUV5 }}$ promoter to generate plasmids PGDT005 and pGDT006, respectively. Sequence fidelity was confirmed by sequencing using the primers GDT_p37_SeqF-R, mifR_seqF-R, mifS_seqF-F2 and PA5530_seqF-R (Table 2.2). Henceforth, these plasmids are referred to as pMifSR and pPA5530.

These expression plasmids were then introduced into wild-type PAO1, $\mathrm{PAO} \Delta$ mifS, $\mathrm{PAO} \Delta$ mifR, $\mathrm{PAO} \Delta$ mifSR and $\mathrm{PAO} \Delta r p o N$ deletion mutants by electroporation (Choi et al., 2006) and gentamycin resistant colonies were selected.

\section{Phenotypic microarray}

Comparative phenotypic microarray profiles of wild-type PAO1 with PAO $\Delta$ mifR and PAO $\Delta$ mifS mutant were performed at BioLOG Inc. (Hayward, CA, USA). Phenotypic profiling was carried out in triplicate and data analyses was done using OmniLog PM Software.

\section{Growth curves}

P. aeruginosa $\mathrm{PAO} 1$ and its derivatives were grown overnight at $37^{\circ} \mathrm{C}$ in LB broth with or without antibiotics. Overnight cultures were washed with sterile $0.85 \%$ $\mathrm{NaCl}(\mathrm{wt} / \mathrm{vol})$ solution to remove spent and residual media. Cultures were diluted in fresh $\mathrm{M9}$ minimal media to obtain equal optical densities $\left(\mathrm{OD}_{600}\right)$ of 0.025 . Growth of the cultures was assessed in LB broth and in M9 minimal media supplemented with glucose $(30 \mathrm{mM})$, sucrose $(30 \mathrm{mM})$ or TCA cycle intermediates including citrate, $\alpha-K G$, succinate, fumarate, malate or oxaloacetate (at $30 \mathrm{mM}$, 
unless specified otherwise) as a sole carbon source in 48 and 96 well plates (Falcon). Growth was monitored by determining absorbance at $600 \mathrm{~nm}$ using BioTek Synergy HT (Winooski, VT, USA) plate reader for $18-24 \mathrm{~h}$ at $37^{\circ} \mathrm{C}$. All experiments were performed multiple times in triplicate.

\section{Pyocyanin and pyoverdine production}

Extracellular pyocyanin was quantified by extracting the pigment from culture supernatants using the chloroform-HCL method as described previously (Essar et al., 1990). Briefly, $5 \mathrm{ml}$ culture supernatants from stationary-phase cultures $(\sim 18 \mathrm{~h})$ grown in King's A medium was extracted with $3 \mathrm{ml}$ chloroform. Pyocyanin was then re-extracted into $1 \mathrm{ml}$ of $0.2 \mathrm{~N} \mathrm{HCl}$, resulting in a pink color, indicating the presence of pyocyanin that was read at $520 \mathrm{~nm}$. The concentration is expressed as $\mu \mathrm{g}$ of pyocyanin produced per $\mathrm{ml}$ of culture $(\mu \mathrm{g} / \mathrm{ml})$, by multiplying the optical density $\mathrm{OD}_{520}$ by 17.072 (Essar et al., 1990).

To measure pyoverdine production, cells were grown overnight at $37^{\circ} \mathrm{C}$ in King's B medium (King et al., 1954). Pyoverdine in the supernatant was read at $405 \mathrm{~nm}$ and normalized to the initial cell density $\left(\mathrm{OD}_{600}\right)$. Pyoverdine levels were expressed as a ratio of $\mathrm{OD}_{405} / \mathrm{OD}_{600}$ (Shen et al., 2002).

\section{Minimum Inhibitory Concentration}

MICs were determined using the E-test as per the manufacturers protocol (BioMerieux, USA) and/or by standard broth microdilution method. The assays were performed in triplicate, each with technical triplicate, for each antibiotic in cation-adjusted Mueller Hinton broth. 


\section{RNA isolation, cDNA synthesis and qRT-PCR}

RNA was isolated from $P$. aeruginosa wild-type PAO1, PAO $\Delta$ mifR, PAO $\Delta$ mifS and PAO $\Delta$ mifSR strains grown in LB broth followed by $1 \mathrm{~h}$ treatment with $30 \mathrm{mM}$ a-KG. Briefly, overnight cultures grown in LB broth at $37^{\circ} \mathrm{C}$ were washed with sterile $0.85 \%$ saline solution to remove spent media and were subcultured at $37^{\circ} \mathrm{C}, 200 \mathrm{rpm}$ in LB media. LB broth was used as a carbon source for initial growth of cultures since PAO $\Delta$ mifR, PAO $\Delta$ mifS, PAO $\Delta$ mifSR and PAO $\triangle r p o N$ strains exhibit growth defects in the presence of $\alpha-K G$ alone. When the cells reached an optical density at $600 \mathrm{~nm}\left(\mathrm{OD}_{600}\right)$ of $0.6-0.7$ all the cultures were treated with $30 \mathrm{mM}$ a-KG for $1 \mathrm{~h}$. Post treatment, RNA was stabilized by addition of phenol-ethanol mixture (Brencic et al., 2009). Stabilized RNA was then isolated using RNeasy Mini Kit (Qiagen, Inc Venio, Limburg, Netherlands) as per manufacturer's protocol. Residual genomic DNA contamination was removed using RQ1 Rnase-free DNase (Promega, Madison, WI, USA) and RNA was repurified using Rneasy Mini Kit (Qiagen, Inc Venio, Limburg, Netherlands). Quality of purified RNA was assessed on a denaturing agarose gel (NorthernMax Gly, Ambion, Life Technologies, Carlsbard, CA, USA) and quantified at $260 \mathrm{~nm}$ (BioTEK, Synergy HT, Winooski, VT, USA). cDNA was then synthesized by annealing NS5 random primers to total purified RNA and subsequent extension was carried out using SuperScript III reverse transcriptase (Invitrogen, Life Technologies, Carlsbard, CA, USA).

qRT-PCR to study expression levels of PA5530 under a-KG induction was performed using Applied Biosystems Step One cycler and detection system with 
PowerSYBR Green PCR MasterMix with ROX (Applied Biosystems, Life Technologies, Carlsbard, CA, USA). In addition RNA was isolated from PAO1, $\mathrm{PAO} \Delta m i f R, \mathrm{PAO} \Delta m i f S$ and $\mathrm{PAO} \Delta$ mifSR strains grown in M9 Minimal media supplemented with citrate $(30 \mathrm{mM})$ without $\alpha-K G$ treatment, as described previously. qRT-PCR to study expression levels of genes encoding sigma-54 rpoN (PA4462), iso-citrate dehydrogenase (idh (PA2623) and icd (PA2624)), a-KG dehydrogenase complex (sucA (PA1585) and Ipd3 (PA4829)) were done essentially as described above. The cycling conditions used were $95^{\circ} \mathrm{C} / 2$ minutes (holding); 40 cycles of $95^{\circ} \mathrm{C} / 15 \mathrm{sec}, 60^{\circ} \mathrm{C} / 1 \mathrm{~min}$ (cycling); $95^{\circ} \mathrm{C} / 15 \mathrm{sec}, 60^{\circ} \mathrm{C} / 1 \mathrm{~min}$, $95^{\circ} \mathrm{C} / 15 \sec \left(0.6^{\circ} \mathrm{C}\right.$ ramp) (melt curve). Expression was normalized to $\mathrm{c} / \mathrm{pX}$ (PA1802), whose expression was determined to remain constant between the samples and conditions tested (Balasubramanian et al., 2012).

\section{Bioinformatic Analyses}

Sequence analyses and domain organization studies were performed using the Simple Modular Architecture Research Tool (SMART) (Schultz et al., 1998) and InterPro domain prediction database (Hunter et al., 2012). mifS $\left(\mathrm{P}_{\text {mifs }}\right)$ and PA5530 $\left(P_{P A 5530}\right)$ promoter analyses and motif search was done using the ensemble learning method SCOPE and GLAM2 (Gapped Local Alignment of Motifs) (Chakravarty et al., 2007, Frith et al., 2008). Multiple sequence alignment was generated using ClustalW2 (http://www.ebi.ac.uk/Tools/msa/clustalw2/) and www.pseudomonas.com (Winsor et al., 2011). 


\section{Statistical Analyses}

All data were analyzed for statistical significance using the Student's $t$-test on GraphPad or Analysis of Variance (ANOVA) with post-hoc testing when appropriate, on IBM SPSS Statistics 22.0 statistical analysis software. Differences were considered to be significant at $p$ - values $<0.05$.

\section{Results}

\section{mifS and mifR are a part of a two-gene operon}

In eubacteria, the genes that encode a $\mathrm{HK}$ and its cognate RR are often linked and are co-transcribed (Stock et al., 1989). Our sequence analysis of $P$. aeruginosa PAO1 genome revealed that mifS (PA5512) and mifR (PA5511) are adjacent to each other, in the same orientation. The predicted translation start site of mifR ORF overlaps with mifS translation termination codon indicating that they are cotranscribed (Figure 2.1A and 2.1B). To determine if these two genes form an operon, cDNA across the intergenic regions spanning mifS and mifR was amplified using GDT_cotransF1-R1 and GDT_cotransF2-R2 primers (see Materials and Methods)x. As expected, $200 \mathrm{bp}$ and $100 \mathrm{bp}$ products were detected when using primers that span the overlapping region (Figure 2.1C, Lane 3 and Lane 4). These results confirm that mifS and mifR are a part of a two-gene operon. As controls, the mifSR genes were also amplified (Figure 2.1C, Lane 2).

\section{Loss of mifS and mifR did not affect antibiotic resistance}

To identify the role of MifSR TCS, clean in-frame deletion mutants of mifS, mifR and mifSR were constructed in the prototypic P. aeruginosa PAO1. Henceforth they will be referred to as PAO $\Delta$ mifS, PAO $\Delta$ mifR and PAO $\Delta$ mifSR, 
respectively. For complementation studies, recombinant plasmids containing the entire mifR, mifS and mifSR genes were constructed. The complementing plasmids with the genes are called pMifS, pMifR and pMifSR. These plasmids were introduced into the respective mutant strains. 


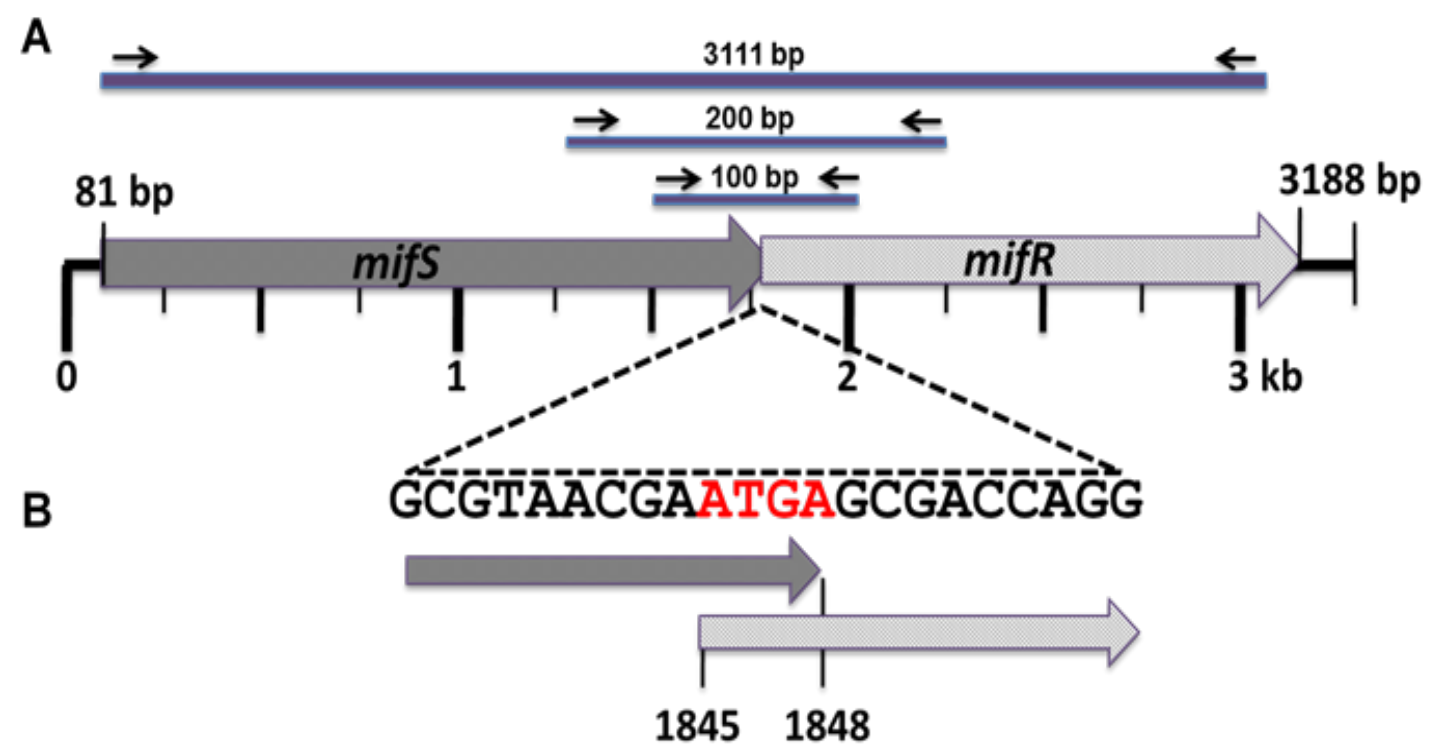

C

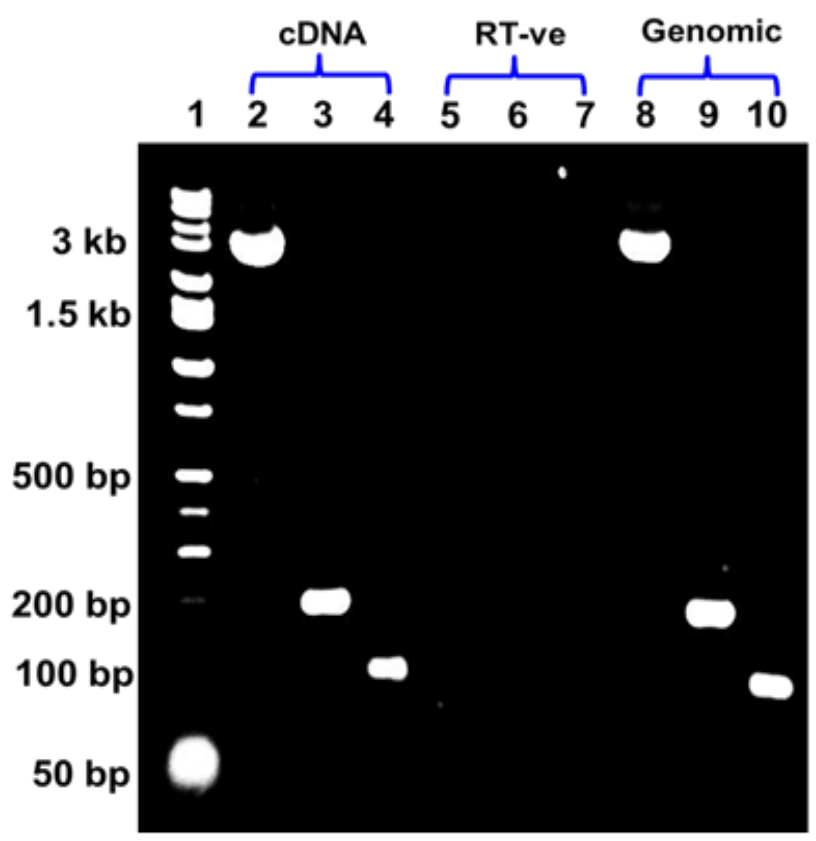

Figure 2.1: Genome organization of the mifSR gene locus. In $P$. aeruginosa PAO1 the mifR (PA5511) ORF has a translation start codon (Briard et al.) overlapping the mifS (PA5512) termination codon (TGA), denoted in red (B), suggesting that the mifS and mifR genes are physically linked. The CDNA amplification of the intergenic region spanning the mifS and mifR genes using GDT_cotrans F1-R1 and GDT_cotrans F2-R2 primers (Table 1) confirm that the two genes mifS and mifR are co-transcribed and form an operon (C). 
Previous studies in our lab postulated that the MifSR TCS system, found 81-bp upstream of the pox operon, may contribute to $P$. aeruginosa ß-lactam resistance (Kong et al., 2005) as the genes regulated by TCS tend to be co-located on the chromosome (Stock et al., 1989). However, MIC analyses using E-test and micro-dilution methods showed that the loss of these genes did not affect the antibiotic resistance profile when compared to the parent strain, $P$. aeruginosa PAO1(Data not shown). Further, qRT-PCR studies showed that deletion of mifS, mifR and mifSR had no effect on the expression of poxB compared to the parent PAO1 (Figure 2.2).

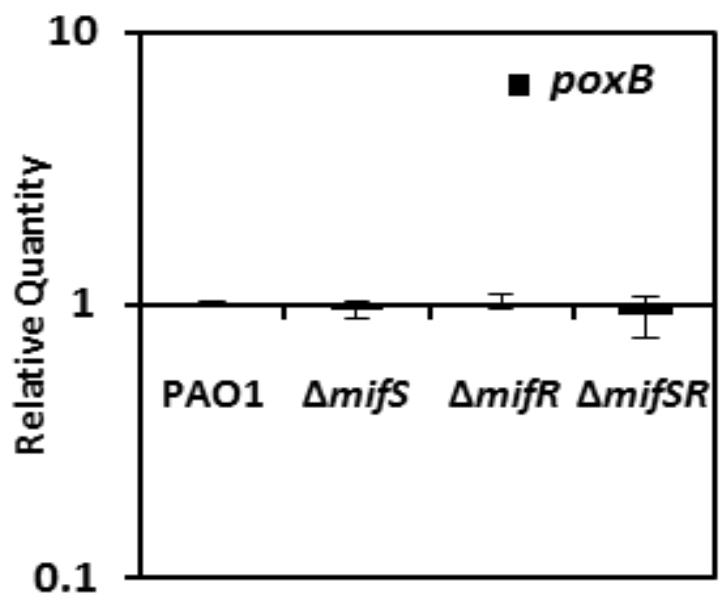

Figure 2:2 Expression of poxB (PA5514) in mifSR mutants. Expression of poxB (PA5514) was tested in mifSR mutants relative to PAO1. Data was normalized to expression in PAO1. Bars above or below the line represents upand down-regulation, respectively and the bars indicate standard errors. The clpX gene (PA1802) was used as the housekeeping control. There was no statistically significant difference ( $p$-value $>0.05$ ) between the wild type PAO1 and mifSR mutant strains as determined by one-way ANOVA and student's unpaired $t$ test. 


\section{mifS, mifR and mifSR mutants failed to grow in the presence of $\alpha-K G$}

The PAO $\Delta$ mifS, PAO $\Delta$ mifR and PAO $\Delta$ mifSR mutants exhibited no discernible phenotype compared to the parent PAO1 when tested for growth, swimming, swarming, twitching motility (LB media), pyocyanin production (LB \& King's A media), pyoverdine production (LB \& King's B Media), congo red binding assay (CR media) and antibiotic resistance (MH media) (Data not shown). Hence, a comparative phenotypic microarray analysis was performed with the wild-type PAO1, PAO $\Delta$ mifR and PAO $\Delta$ mifS mutants (BioLOG Inc.). Out of approximately 2000 metabolic and chemical sensitivity assays tested, PAO $\Delta$ mifR exhibited four gain-of-function and 29 loss-of-function phenotypes whereas PAO $\Delta$ mifS exhibited two gain-of-function and 23 loss-of-function phenotypes (Figure 2.3A). A single gain of function phenotype shared between $\mathrm{PAO} \Delta$ mifS and $\mathrm{PAO} \Delta$ mifR, was the ability to utilize L-methionine. When metabolism and chemical sensitivity were compared, the mutants appear more sensitive to various antibiotics (Figure 2.3B). However, none of these were reproducible in the lab in the MH media. The loss of mifS and mifR resulted in differential phenotype in the presence of six metabolites, amongst which, two were common to both mifS and mifR mutants (Figure 2.3B). The shared metabolic phenotypes involved the utilization of L-methionine and $\alpha$ KG (Figure 2.3C). Compared to the parent PAO1, the mutants did not exhibit any growth increase when provided with L-methionine (Figure 2.4). This could be simply due to the difference in culture conditions and BioLOG proprietary media.

The inability to utilize $\alpha-K G$ by $\mathrm{PAO} \Delta$ mifS (Figure $2.5 \mathrm{~A}$ ) and PAO $\Delta$ mifR (Figure 2.5B) in the BioLOG assay was reproduced in M9 minimal media 
supplemented with $30 \mathrm{mM}$ a-KG (Figure 2.5C). In fact, all three mutant strains, $\mathrm{PAO} \Delta m i f R, \mathrm{PAO} \Delta$ mifS and $\mathrm{PAO} \Delta$ mifSR failed to grow in the presence of $\alpha-K G$ (Figure 2.5C). To rule out potential toxicity, the wild-type $P$. aeruginosa PAO1 and the mutants were cultured in M9 minimal media with varying concentrations of $\alpha$ $K G$, ranging from 1 to $80 \mathrm{mM}$ (Figure 2.6). The mutants exhibited no growth in the presence $\alpha-K G$ after $24 \mathrm{~h}$ at $37^{\circ} \mathrm{C}$, whereas the wild-type PAO1 exhibited an increase in growth that was proportional to $\alpha-K G$ concentration (Figure 2.6B). All subsequent experiments were done with $30 \mathrm{mM} \alpha-K G$. The growth defect exhibited by $\mathrm{PAO} \Delta$ mifS, PAO $\Delta$ mifR and PAO $\Delta$ mifSR could be restored to the wild-type levels by introducing mifR and mifSR genes into the respective mutants (Figure 2.5D and Figure 2.7A). 

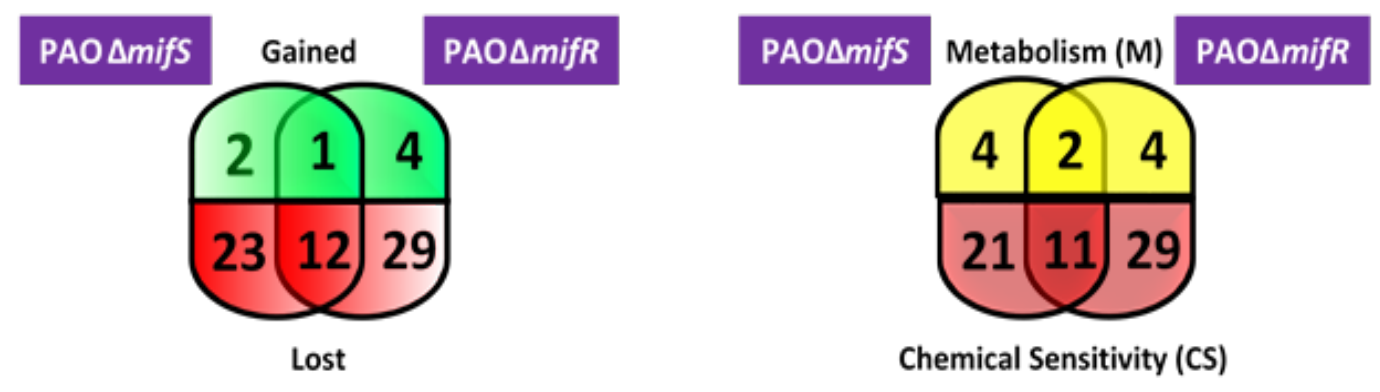

C

\begin{tabular}{|l|l|l|}
\hline Substrate & Nature of substrate & Role \\
\hline Phenotypes Gained & \multicolumn{2}{|c|}{} \\
\hline L-Methionine & S-source, Nutritional supplement & M \\
\hline Phenotypes Lost & \multicolumn{1}{|l}{} \\
\hline a-Ketoglutaric acid & C-Source, carboxylic acid & M \\
Vancomycin & Wall & CS \\
Sulfadiazine & Folate antagonist, PABA analog & CS \\
Ribostamycin & Protein synthesis, 30S ribosomal subunit, aminoglycoside & CS \\
5- Fluorouracil & Nucleic acid analog, pyrimidine & CS \\
Sodium metavanadate & Toxic anion, PO4 analog & CS \\
Sodium orthovanadate & Toxic anion, PO4 analog & CS \\
2- Nitroimidazole & Nitro compound, oxidizing agent, ribonucleotide DP reductase inhibitor & CS \\
Cefotaxime & Wall, cephalosporin & CS \\
Norfloxacin & DNA topoisomerase & CS \\
Streptomycin & Protein synthesis, 30S ribosomal subunit, aminoglycoside & CS \\
Coumarin & DNA intercalator & CS \\
\hline
\end{tabular}

Figure 2.3: mifS and mifR dependent phenotypes. To identify the role of $P$. aeruginosa mifSR TCS, comparative phenotypic microarray of PAO $\triangle$ mifS, PAO $\triangle$ mifR mutants and wild-type PAO1 strain was performed at BioLOG Inc. (Hayward, CA, USA). Venn diagram of differentially regulated phenotypes of the mutants compared to their isogenic parent PAO1, showing gain of function or loss of function phenotypes (A). Phenotypic differences were further classified based on metabolic and chemical sensitivity properties (B). The phenotypes common to both mifS and mifR mutants are listed (C). 


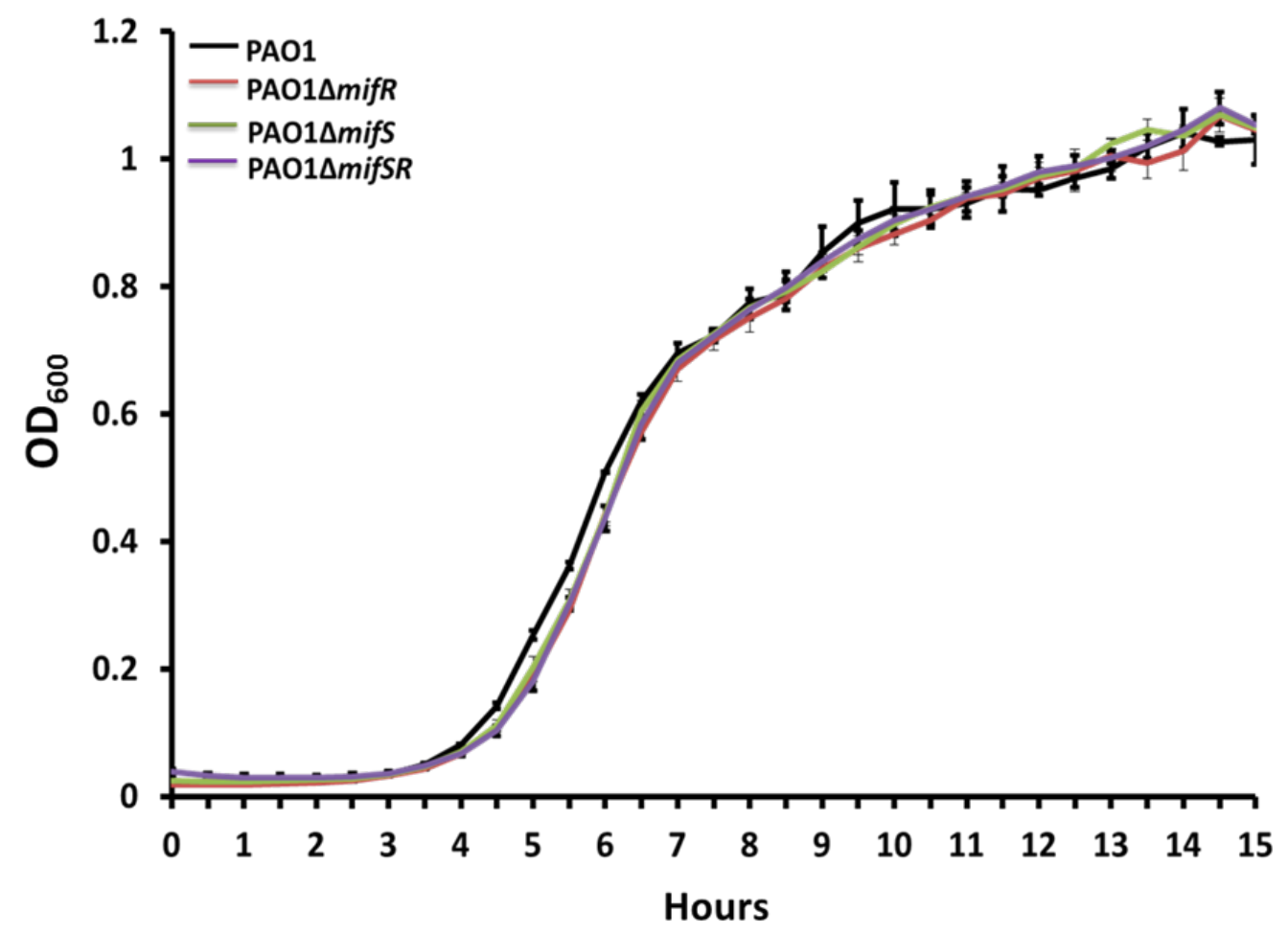

Figure 2.4: Growth curve analysis in the presence of methionine. Growth curves of $P$. aeruginosa wild-type PAO1 and mifSR mutants in M9 minimal media supplemented with glucose $(30 \mathrm{mM})$ and methionine $(5 \mathrm{mM})$ as carbon and nitrogen source. 
A B

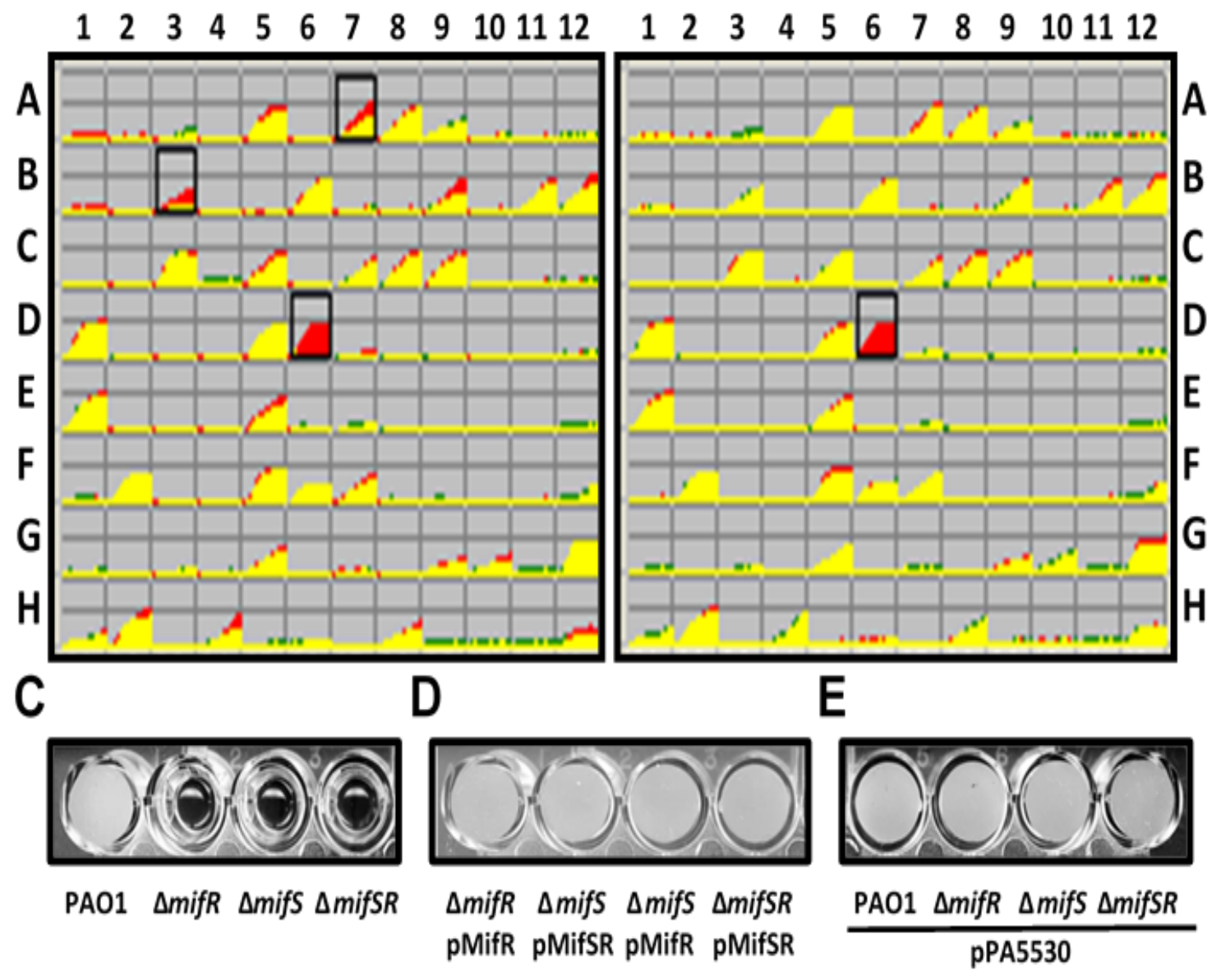

Figure 2.5: Phenotypic microarrays of PAOAmifS and PAOAmifR mutants.

The loss of mifS and mifR results in a growth deficient phenotype in the presence of $\alpha-K G$ as a sole carbon source, as depicted by BioLOG plate PM1, well D6 ( $A$ and $B$ ). Loss of growth phenotype was confirmed by growing PAO1, $\mathrm{PAO} \Delta$ mifS, PAO $\Delta$ mifR and $\mathrm{PAO} \Delta$ mifSR mutants in M9 minimal media with $\alpha$ KG (30 mM) for 18 to $24 \mathrm{~h}$ at $37^{\circ} \mathrm{C}(\mathrm{C})$. The growth defect was rescued by expressing mifR and mifSR genes (D) and the gene encoding the $\alpha-K G$ specific transporter PA5530 (E) in trans. 


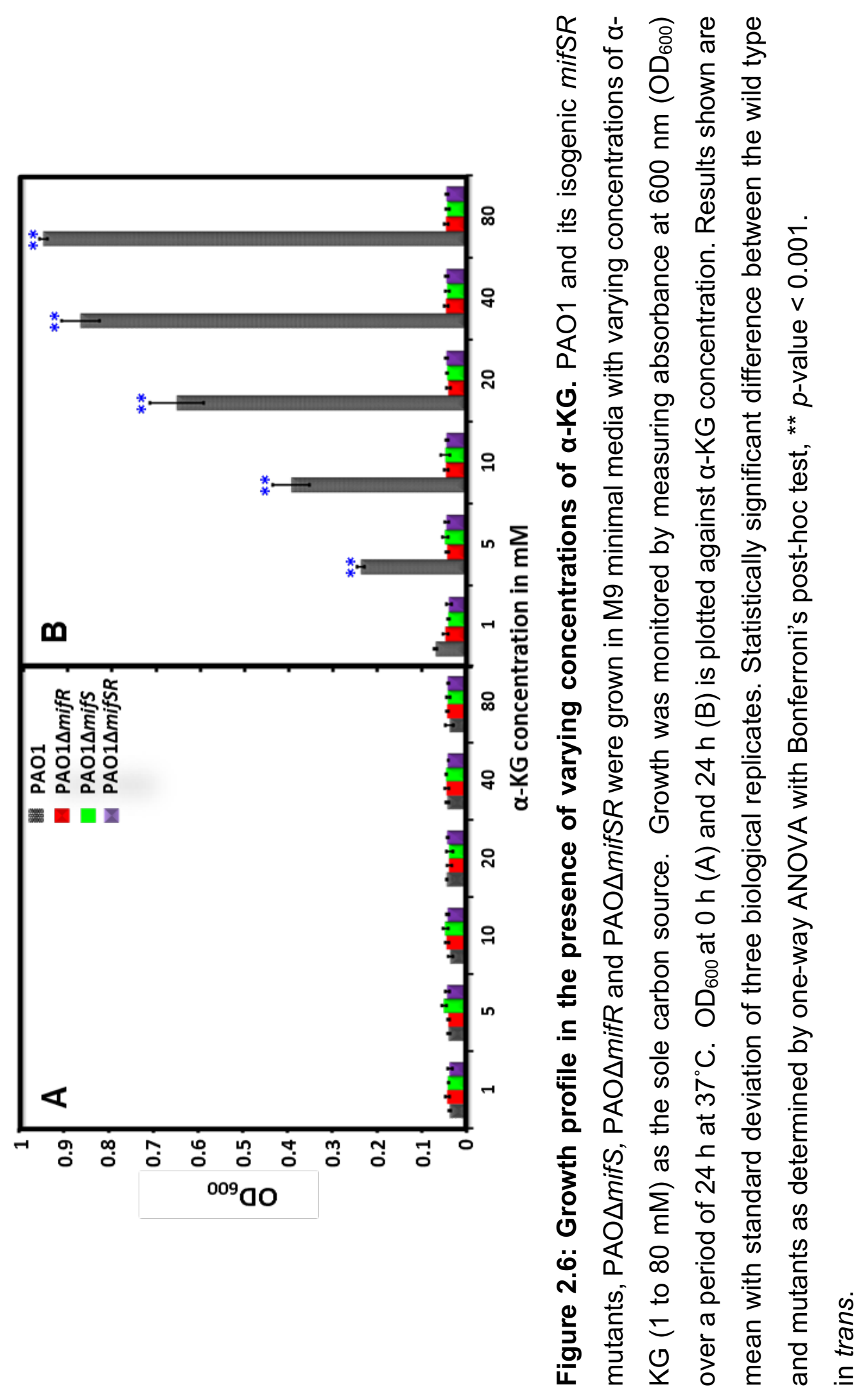



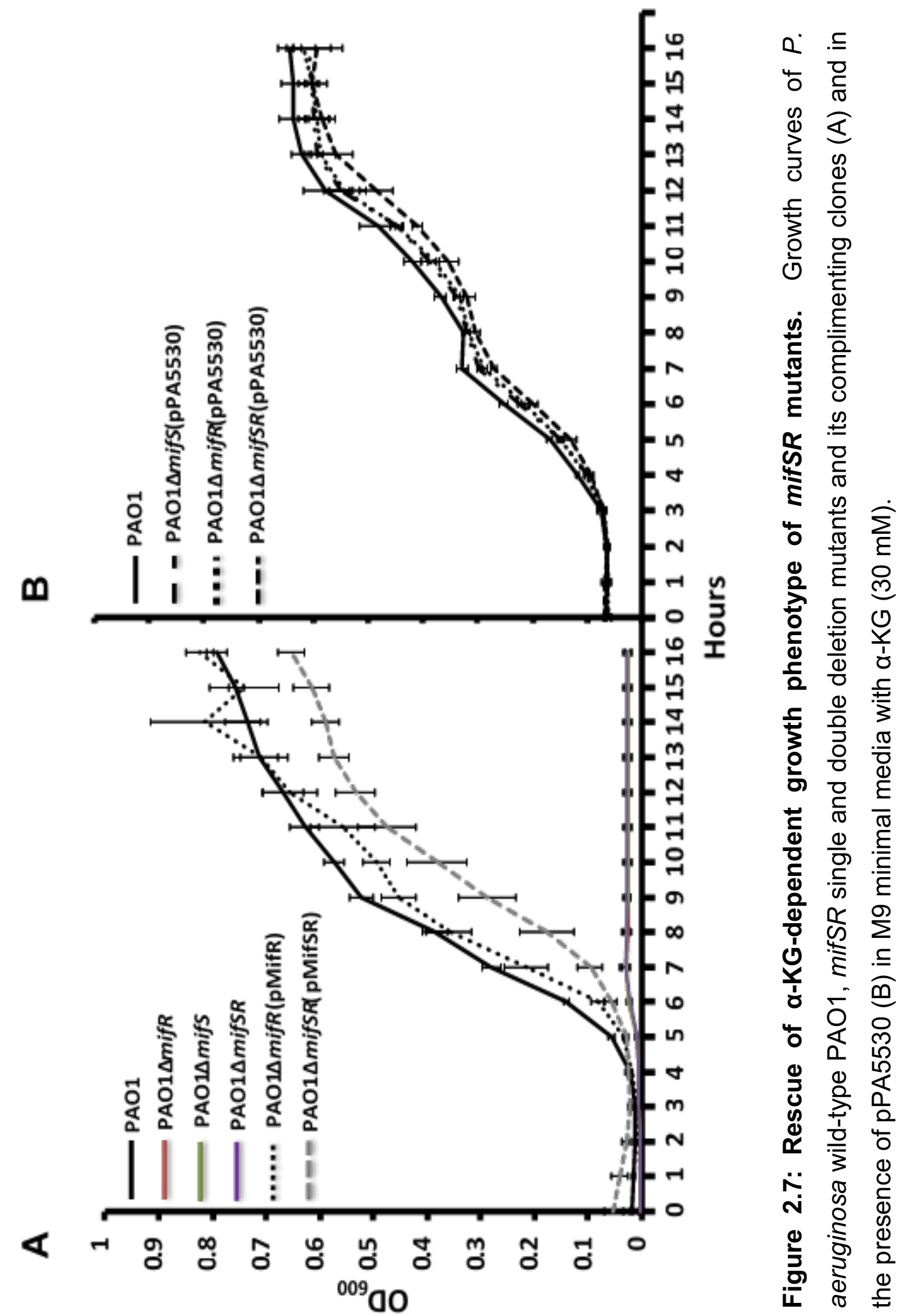


\section{mifSR mutants exhibit $\alpha-K G$ dependent growth defect}

a-KG is a key TCA cycle intermediate (Figure 2.8) and plays an important role in regulating carbon and nitrogen metabolism (Doucette et al., 2011). It has been previously shown that $P$. aeruginosa preferentially utilizes TCA cycle intermediates as a carbon source over other compounds (Liu, 1952, Wolff et al., 1991, Suh et al., 2002). To test if the growth defect exhibited by the loss of mifS and mifR is restricted to $\alpha-K G$ utilization, the mutants and the complementing strains were grown in the presence of TCA cycle intermediates citrate, succinate, fumarate, malate and oxaloacetate at $30 \mathrm{mM}$ each. No difference in growth was observed between wild type PAO1 and its isogenic mutants in the presence of other TCA cycle intermediates except for $\alpha-K G$ (Table 2.3). This is not surprising as $P$. aeruginosa can use the glyoxylate shunt pathway to bypass the need for $\alpha-$ KG (Figure 2.8) (Campbell et al., 1953). Furthermore, no difference in the growth profile of the wild type PAO1 and mifSR mutants was observed when grown in the presence of sugars, glucose and sucrose (30 mM each) (Data not shown). To reconfirm that the presence of $\alpha-K G$ is not toxic, the cells were grown in the presence of citrate and succinate combined in equal concentration with $\alpha-K G$. The mutants and the wild type shared similar early exponential growth (Figure 2.9). However, the mutants reached stationary phase earlier as compared to the parent strain PAO1. This suggests that the presence of excess carbon source in the form of $\alpha-K G$ further contributes to the growth of PAO1. These analyses indicate that mifSR mutants are only defective in $\alpha-K G$ utilization. 


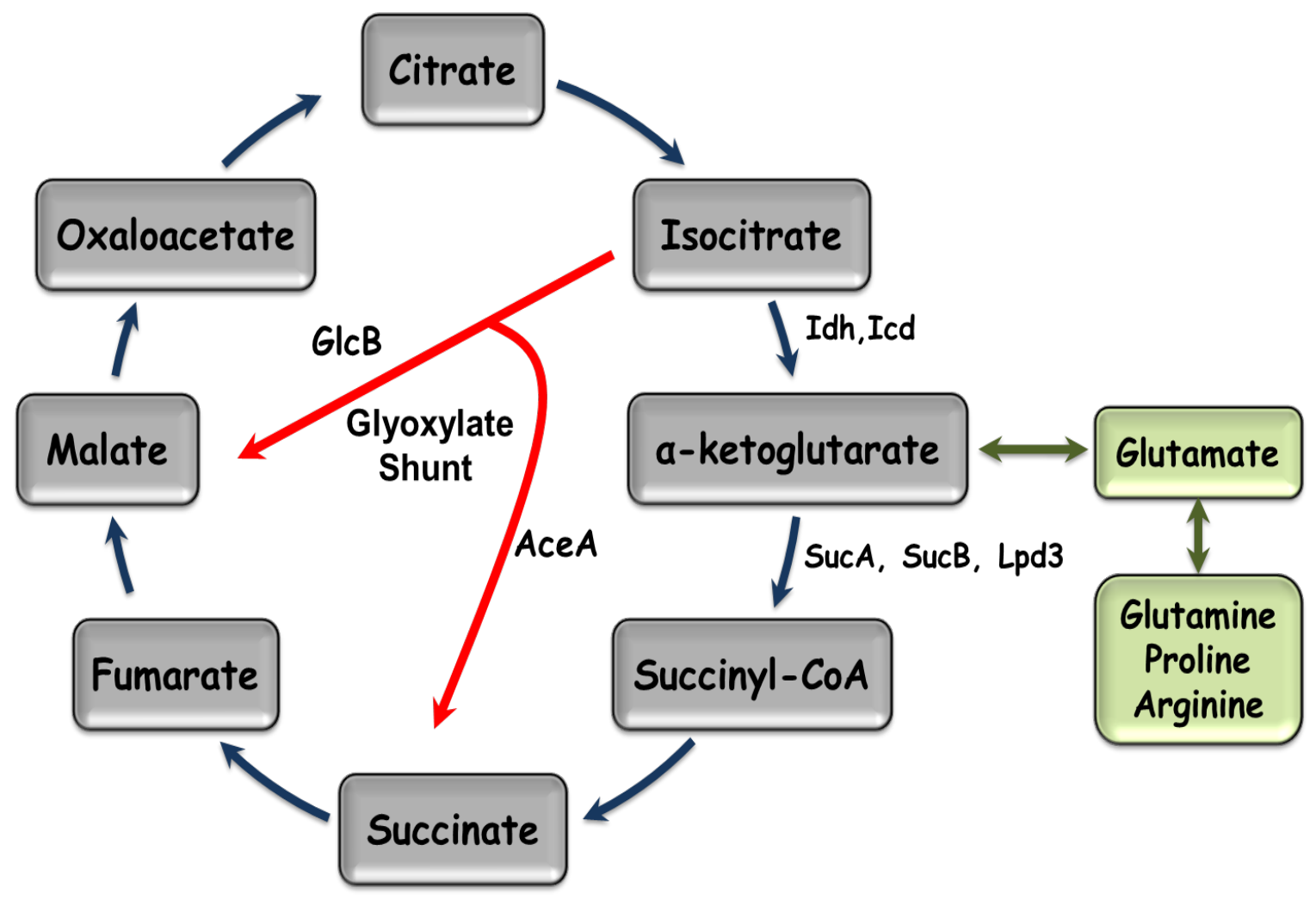

Figure 2.8: Tricarboxylic acid (TCA) cycle and its related reactions. Enzymes converting iso-citrate to $\alpha-K G$ (iso-citrate dehydrogenase: Icd, Idh), $\alpha-K G$ to succinyl-coA ( $\alpha-K G$ dehydrogenase complex: SucA, SucB, Lpd3) and those involved in the glyoxylate shunt (isocitrate lyase (AceA) and malate synthase $G$ $(G \ln B))$ are shown in bold. Green boxes indicate the amino acid biosynthetic precursors of $\alpha-K G$ involved in the anaplerotic reaction. 


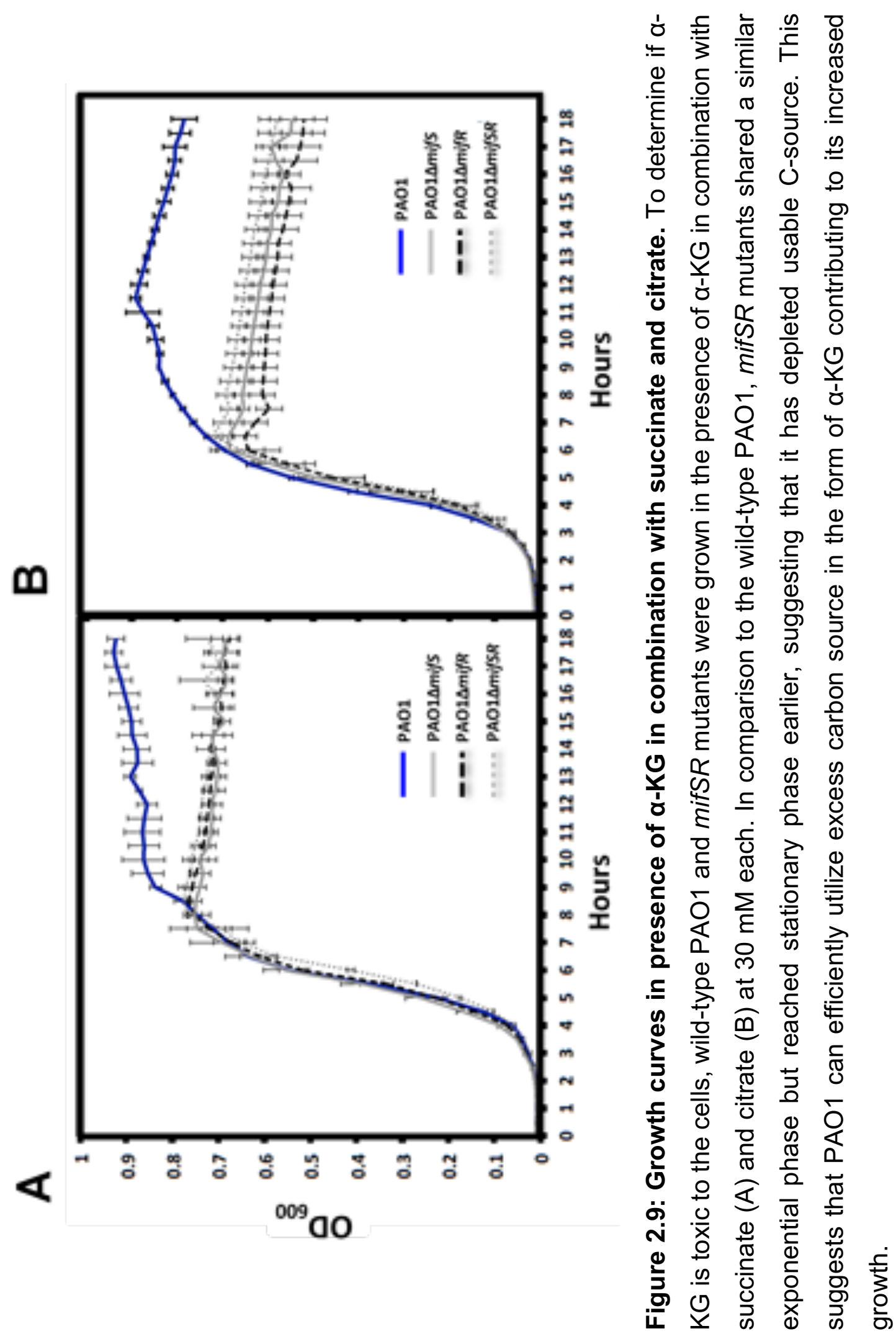




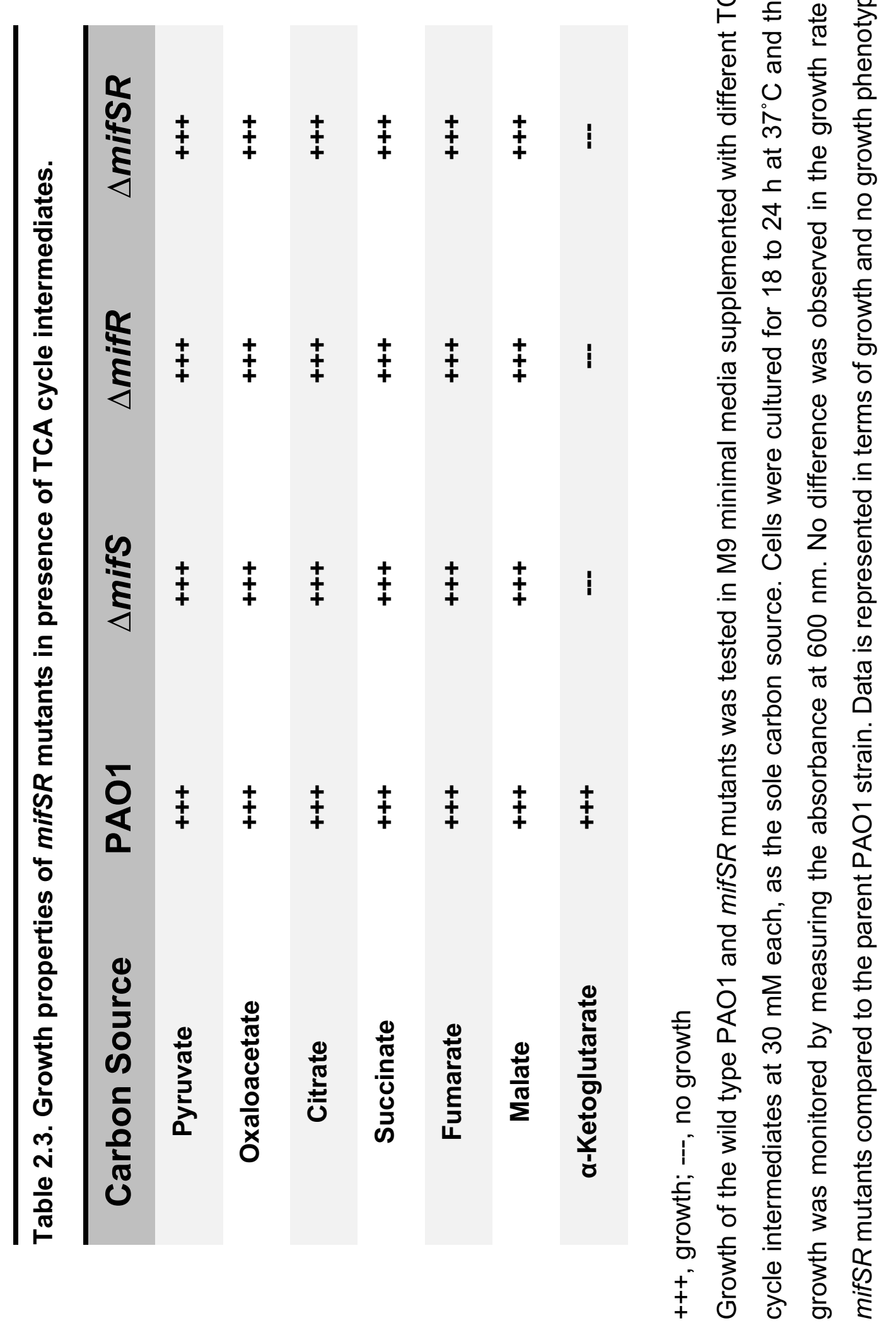




\section{mifSR mutants are defective in $\alpha-K G$ transport}

The absence of growth in the presence of exogenous $\alpha-K G$ could be due to either failure to enter the cells or loss of the mutants' ability to convert $\alpha-K G$ to succinate. The latter is likely if the mutants failed to express a functional $\alpha-K G$ dehydrogenase complex. The ability of mifSR mutants to grow effectively in the presence of citrate and succinate suggests that these mutants are likely to harbor a functional $\alpha-K G$ dehydrogenase complex, unless the mutants bypass it using the glyoxylate shunt (Figure 2.8). The former is likely as qPCR analysis of genes encoding isocitrate dehydrogenase (idh, icd) and $\alpha-K G$ dehydrogenase complex (sucA, $s u c B, I p d 3$ ) revealed no difference in the expression levels in the wild-type PAO1 and mifSR mutants (Figure 2.10).

a-KG is a hub for anaplerotic reactions, a process for replenishing TCA cycle intermediates. In this process glutamate, glutamine, proline and arginine act as precursor molecules for $\alpha-K G$ synthesis (Kornberg, 1966). Growth studies in the presence of these amino acids would serve as another indirect measure to test the functionality of $\alpha-K G$ dehydrogenase complex in mifSR mutants. To test this hypothesis, PAO1, PAO $\Delta$ mifR, PAO $\Delta$ mifS and PAO $\Delta$ mifSR mutants were cultured in the presence of glutamate, glutamine, proline and arginine (Table 2.4). The parent PAO1 and the isogenic mutants exhibited similar growth phenotype. From the expression studies and growth analyses we deduce that the mifSR mutants are impaired in $\alpha-K G$ transport. 


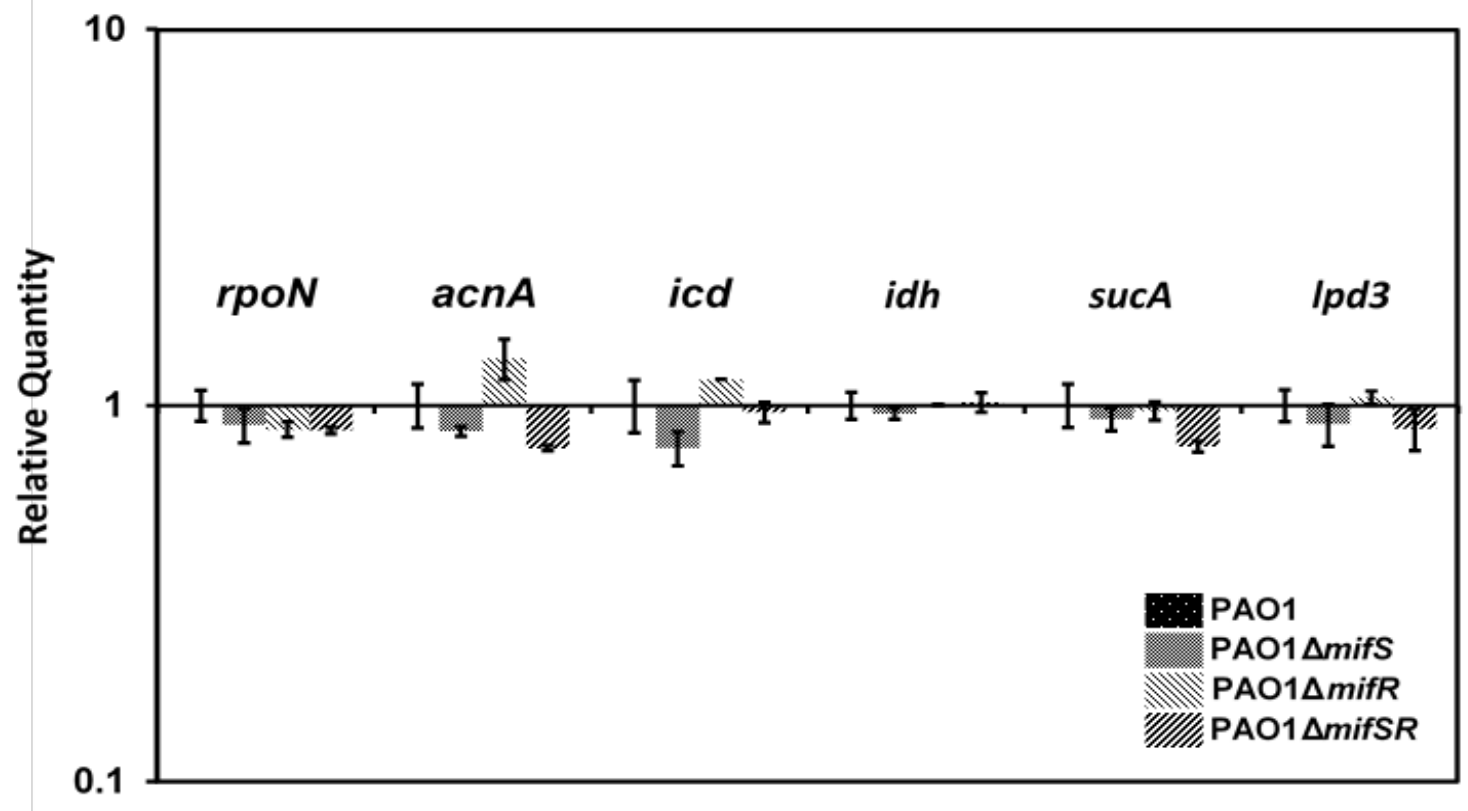

Figure 2.10: Quantification of $r p o N$, acnA, idh, icd, sucA, and Ipd3 mRNA by qRT-PCR. RNA was isolated from cells grown in M9 minimal media supplemented with citrate $(30 \mathrm{mM})$, reverse transcribed to cDNA and the presence of specific transcripts was analyzed by qPCR using gene-specific primers (Table 5). The expression of genes encoding aconitate hydratase 1 (acnA (PA1562)) isocitrate dehydrogenase (idh (PA2623)) isocitrate dehydrogenase, $\alpha-K G$ dehydrogenase complex (icd (PA2623)), sucA (PA1585) and Ipd3 (PA4829), and $\sigma^{54}$ (rpoN (PA4462)) were analyzed in mifSR mutants relative to PAO1 $\left(\log _{10} R Q=1\right)$. Bars above or below the line represents upand down-regulation, respectively and the bars are standard errors. The $c / p X$ (PA1802) gene was used as the housekeeping control. Statistically significant difference between the wild type and mutants as determined by one-way ANOVA with Bonferroni's post-hoc test. Difference in the expression levels of genes is not statistically significant at $p$-value $<0.05$. 
Table 2.4. Growth profile analysis of the mifSR mutants in presence of amino acids.

\begin{tabular}{ccccc}
\hline Carbon Source & PAO1 & $\Delta$ mifR & $\Delta$ mifS & $\Delta$ mifSR \\
Glutamate & +++ & +++ & +++ & +++ \\
Glutamine & +++ & +++ & +++ & +++ \\
Proline & +++ & +++ & +++ & +++ \\
Arginine & +++ & +++ & +++ & +++ \\
\hline
\end{tabular}

+++ , growth; ---, no growth

Cells were grown in the M9 minimal media with the indicated amino acids (30 $\mathrm{mM}$ each). Data is represented in terms of growth and no growth phenotype.

mifSR TCS genes regulate extracellular $\alpha-K G$ transport

In a recent study using transposon mutagenesis; PA5530 was identified as the functional a-KG transporter (Lundgren et al., 2014). To confirm the role of $P$. aeruginosa $P A 5530$ in a-KG uptake and identify the role of mifSR genes, the gene was amplified and subcloned downstream of the inducible $\mathrm{P}_{\text {lacuv5 }}$ promoter. The plasmid pPA5530 was introduced into PAO1 and the mifSR mutants. Expression of $P A 5530$ in trans in PAO $\Delta$ mifS, PAO $\Delta$ mifR, PAO $\Delta$ mifSR mutants restored their growth to a level similar to the wild-type PAO1 in M9 minimal media with a-KG (30 $\mathrm{mM}$ ) as the sole carbon source (Figure 2.7B). Expression of an extra copy of PA5530 gene in the wild-type PAO1 did not affect its growth (Figure 2.5E). This finding suggests that expression of $P A 5530$ is likely regulated by MifSR and/or $\alpha$ KG. In fact, expression of PA5530 is regulated by $\alpha-K G$, as seen in qRT-PCR analysis when PAO1 was grown in M9 media with varying amounts $\alpha-K G$ (Figure 2.11A). The loss of mifS, mifR and mifSR results in a significant decrease in PA5530 expression as compared to the wild type PAO1 in the presence of $\alpha-K G$ (Figure 2.11B). Thus, a-KG-dependent PA5530 expression requires MifSR. 


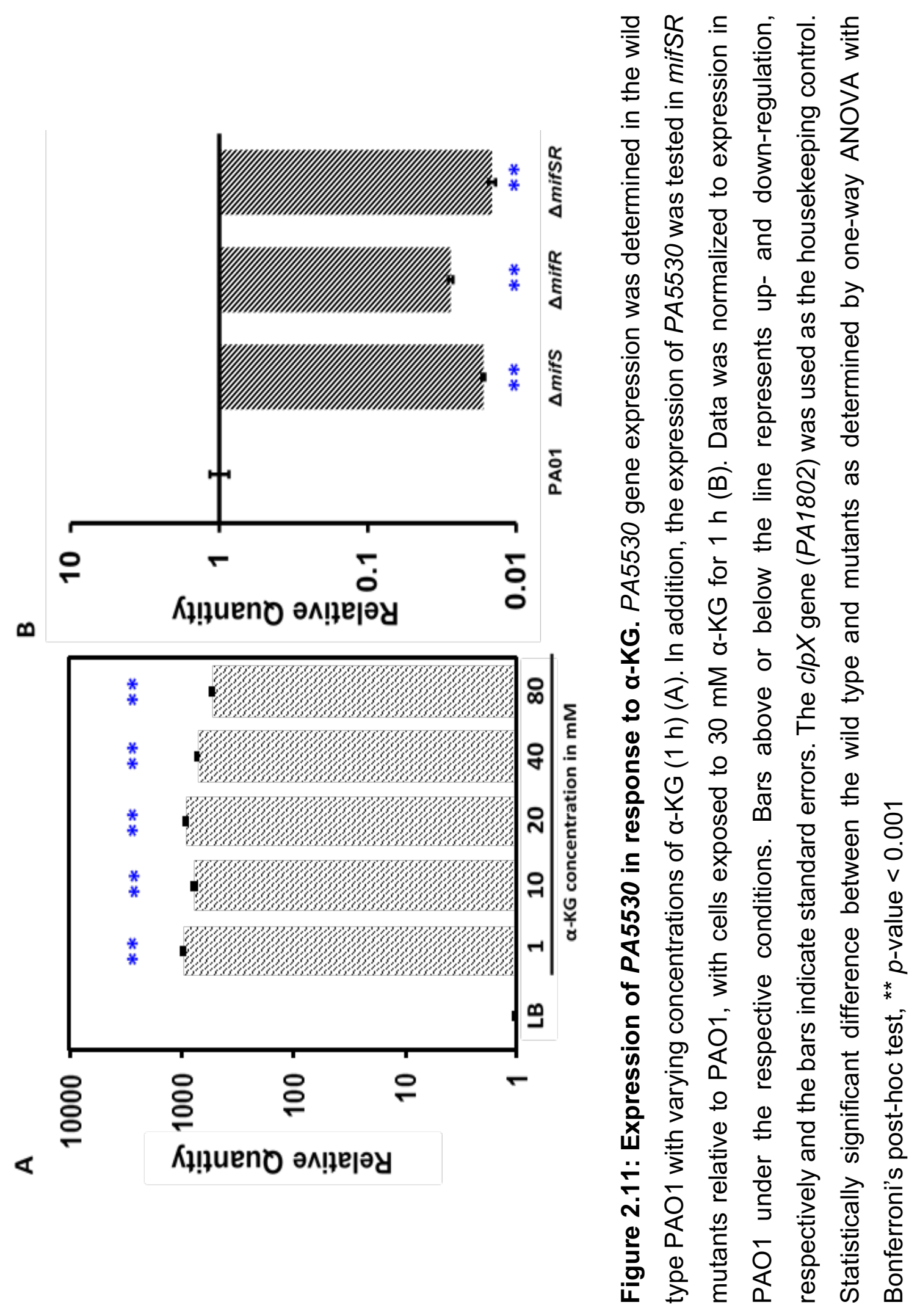




\section{$\operatorname{RpoN}\left(\sigma^{54}\right)$ is required for $\alpha-K G$ utilization}

The closest $P$. aeruginosa MifS and MifR homologs are R. meliloti DctB and DctD (Stover et al., 2000). In fact, MifR is $69 \%$ similar to $R$. meliloti DctD that belongs to the Sigma $54\left(\sigma^{54}\right)$ dependent NtrC family of transcriptional regulators (Stover et al., 2000, Valentini et al., 2011). Thus, it is likely that MifR has the conserved domains found among NtrC family of regulators, an N-terminal regulatory, a central $\sigma^{54}$ activation and a C-terminal DNA binding domains (Morett \& Segovia, 1993, Osuna et al., 1997). MifR analysis using the simple modular architecture research tool (SMART) (Schultz et al., 1998) and InterPro (Hunter et al., 2012) revealed the presence of three domains: CheY-homologous receiver/regulatory, a central $\mathrm{AAA}^{+}$region required for $\sigma^{54}$ activation, and the DNA binding helix-turn-helix domains (Figure 2.12A). The central $A A A^{+}$domain contains seven conserved regions designated C1 to C7 (Morett \& Segovia, 1993) that are characteristic of $\sigma^{54}$-dependent transcriptional regulators. Sequence analysis of MifR revealed the presence of all the seven conserved regions in the $\mathrm{AAA}^{+}$domain between amino acid residues 144 to 373 (Figure 2.12B).

Since MifR exhibits high identity to $\sigma^{54}$-dependent transcriptional regulators, we hypothesized that $P$. aeruginosa rpoN mutants should exhibit a $\alpha-K G-$ dependent phenotype, similar to the mifSR mutants. To verify this hypothesis, we tested the ability of PAO $\triangle r p o N$ mutant to grow in the presence of $\alpha-K G(30 \mathrm{mM})$ (Table 2.5). As expected, PAO $\Delta r p o N$ failed to grow in the presence of $\alpha-K G$ (Table 2.5). The growth of the $r p o N$ mutant was restored in PAO $\Delta r p o N:: r p o N$ complementing strain. Further, in trans expression of mifR and mifSR in 
PAO $\triangle r p o N$ mutant failed to restore their growth in the presence of $\alpha-K G$ (Table 2.5). This data confirms that MifR regulatory function requires functional RpoN $\left(\sigma^{54}\right)$.

The small 81-bp mifSR promoter has no obvious RpoN sigma factor -12/24 consensus sequence: 5'-TGGCACG-N4-TTGCW-3' in which W stands for either $\mathrm{A}$ or $\mathrm{T}$ (Figure 2.13A) (Barrios et al., 1999). In fact, it appears to have a potential -10 (consensus: TATAAT) but lacked -35 (consensus: TTGACA) for sigma-70 promoter (Figure 2.13A) (Paget \& Helmann, 2003). On the other hand, the promoter region of PA5530 is 315-bp long with strong -12 and -24 boxes upstream of the predicted transcription start site (Figure 2.13B). We hypothesized that the inability of $r p o N$ mutant to utilize $\alpha-K G$ can be rescued by expressing PA5530 under a regulatable promoter $\mathrm{P}_{\text {lacUV5. }}$. As expected, the growth of the rpoN mutant was restored when the plasmid harboring the transporter PA5530 was expressed in trans (Table 2.5). This suggests that expression of PA5530 requires both MifSR TCS and RpoN.

The presence of a common motif, GATCGGCGGATt/gTCC, in the $\mathrm{P}_{\text {mifs }}$ and $\mathrm{P}_{\text {PA5530 }}$ (Figure 2.13A and 2.13B) suggest that these two operons share some common regulatory mechanism. In addition, both promoters possess multiple motifs: $P_{\text {mifs }}$ has two sets of large overlapping inverted repeats, and $P_{P A 5530}$ has three sets of direct repeats (Figure 2.13A and 2.13B). However, the role of these motifs remains to be elucidated. 
A

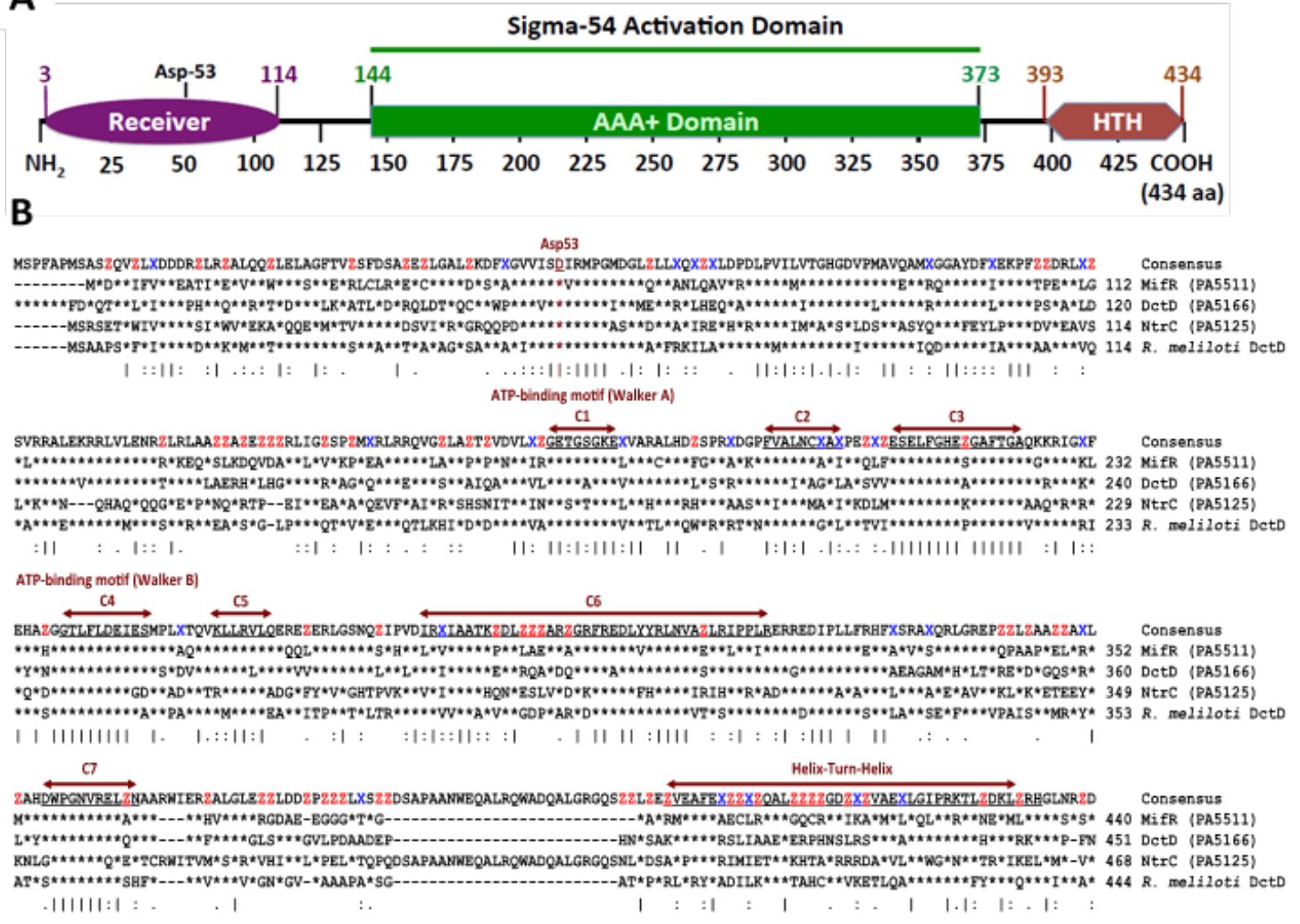

Figure 2.12: $P$. aeruginosa MifR domain organization and sequence

alignment. (A) MifR domain organization determined using the Simple Modular Architecture Research Iool (SMART) (Schultz et al., 1998). MifR is a sigma-54 dependent transcriptional activator (Winsor et al., 2011). There are three functional domains, $\mathrm{N}$-terminal receiver with the conserved aspartate residue at position 53 (Asp-53) (Purple), central AAA ${ }^{+}$ATPase, characteristic of sigma-54 dependent activation proteins (Green), and the C-terminal helix-turn-helix (HTH) DNA binding (Manfredi et al.) domains. (B) Sequence alignment of MifR with $P$. aeruginosa DctD (PA5166), NtrC (PA5125) and $R$. meliloti DctD. Vertical bars indicate conserved residues, asterisk $\left(^{*}\right)$ indicate residues are identical at that position. Key residues of the central $\mathrm{AAA}^{+}$domain ( $\mathrm{C} 1$ to $\mathrm{C} 7$ ) are well conserved amongst sigma-54 dependent transcriptional activators. The horizontal arrow bars indicate HTH domain. Asp-53 indicates the conserved phosphorylation site of $P$. aeruginosa MifR. The alignment was generated using ClustalW2 (http://www.ebi.ac.uk/Tools/msa/clustalw2/). 
Table 2.5. Growth properties of PAO1 $\Delta r p o N$ and its derivatives in the presence of $\alpha-K G$ and LB.

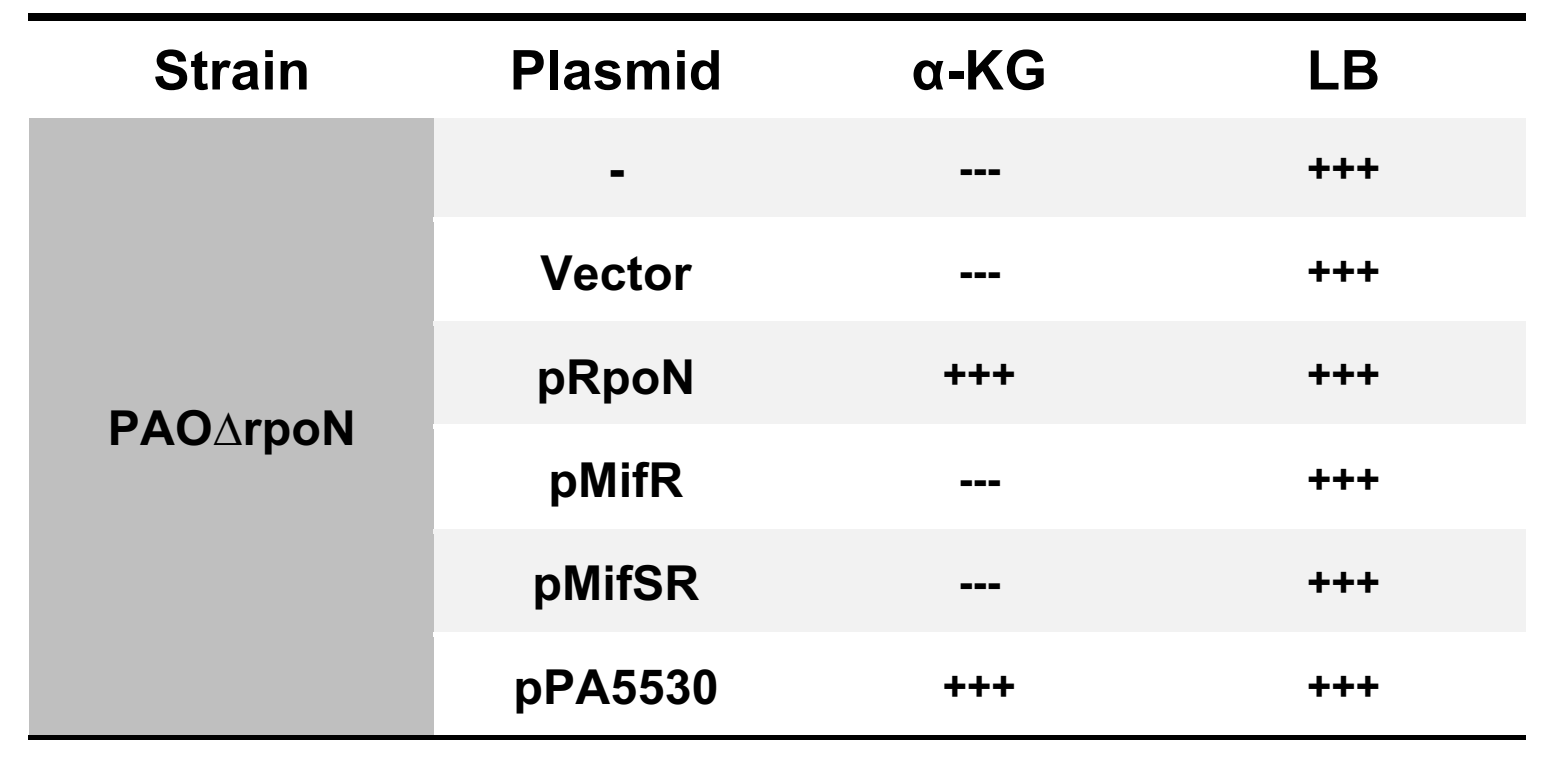

+++, growth; ---, no growth

Growth of PAO1 $\triangle r p o N$ mutant and its derivatives was tested in the M9 minimal media supplemented with $\alpha-K G(30 \mathrm{mM})$ and in the $L B$ media at $37^{\circ} \mathrm{C}$ for $24 \mathrm{~h}$. 


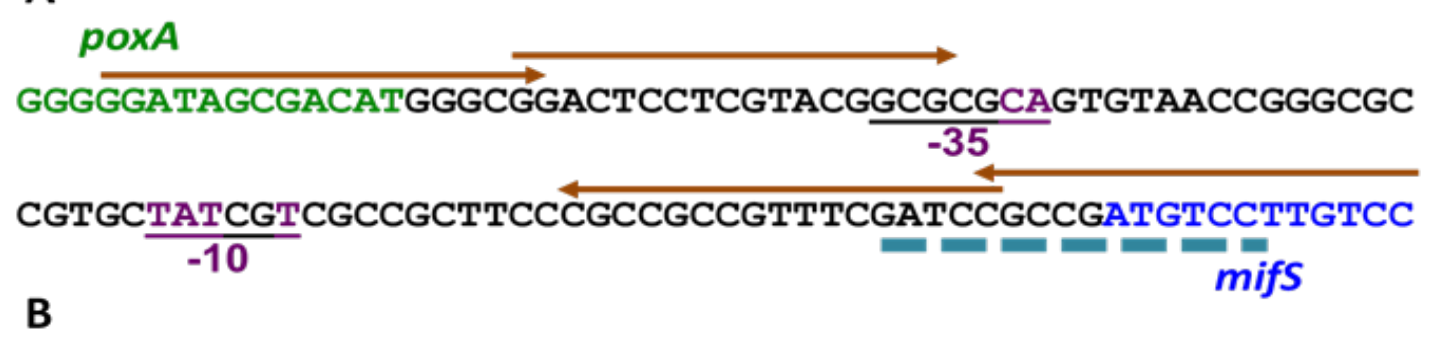

GAGCCAAGCGATCCCGGCCTGCCGTCGAATCGGCGGATAACCACGCTGCGG TTATTCGCCСTCGCCTGCACCTGGTGTCGCTATCGGACССTTTGCCCTGAA GGATCGGCGGATTTCCGCTTATCCACACTCCCGGTCGGCGGACTCCСGCTC GGCCAAGACCACCССGСTCСTCTTCCAACCCAGTCCCCACGCGGCTTTCCC GCGTTGGCACGGCACCTGCTATCGAGCCATCCAATACGTCGCCCTCGCGAT $-24$ $-12$ $+1$ GTGCCGGTGTGCTGCGCCCTGATCAGCACATCCAAGACAACAACGAAAAGA GGAAACACGATGGAAAGCGCCAACGCCATTTCCGCGTCTGCCCAGCCCCGC PA5530

Figure 2.13: In silico analysis of mifS $\left(P_{\text {mifs }}\right)$ and PA5530 ( $\left.P_{P A 5530}\right)$ promoter sequences. Motif search was done using the ensemble learning method SCOPE and GLAM2 (Gapped Local Alignment of Motifs) (Chakravarty et al., 2007, Frith et al., 2008). (A) Sequence analysis of the 81-bp $\left(P_{\text {mifs }}\right)$ (black) indicates a putative $\sigma^{70}$-dependent -10 consensus (TATAAT). However, it lacks the -35 consensus (TTGACA) for $\sigma^{70}$ promoter (Potvin et al., 2008). Arrows represent the long 17-bp direct and inverted repeats in $\mathrm{P}_{\text {mifs }}$ with a consensus GGAt/cAGCGACATCGGCG. (B) The 315-bp promoter region of PA5530 showing strong -12 and $-24 \sigma^{54}$-dependent promoter like element and the proposed transcription start site $(+1)$. Dashed line (blue) depicts a common motif in $\mathrm{P}_{\text {mifs }}$ and $\mathrm{P}_{P A 5530}$ suggesting a common regulatory mechanism ( $\mathrm{A}$ and $B)$. The three pairs of direct repeats in $\mathrm{P}_{P A 5530}$ are represented by green, blue and orange arrows. $P_{P A 5530}$ possess the signature sequence (AAc/uAAc/uAA) for catabolite repression control ( $\mathrm{Crc}$ ) protein (brown box) (Sonnleitner et al., 2009). The uncharacterized small antisense RNA (asRNA) identified in the $P_{\text {PA5530 }}$ region (Wurtzel et al., 2012) is indicated by marked line. 


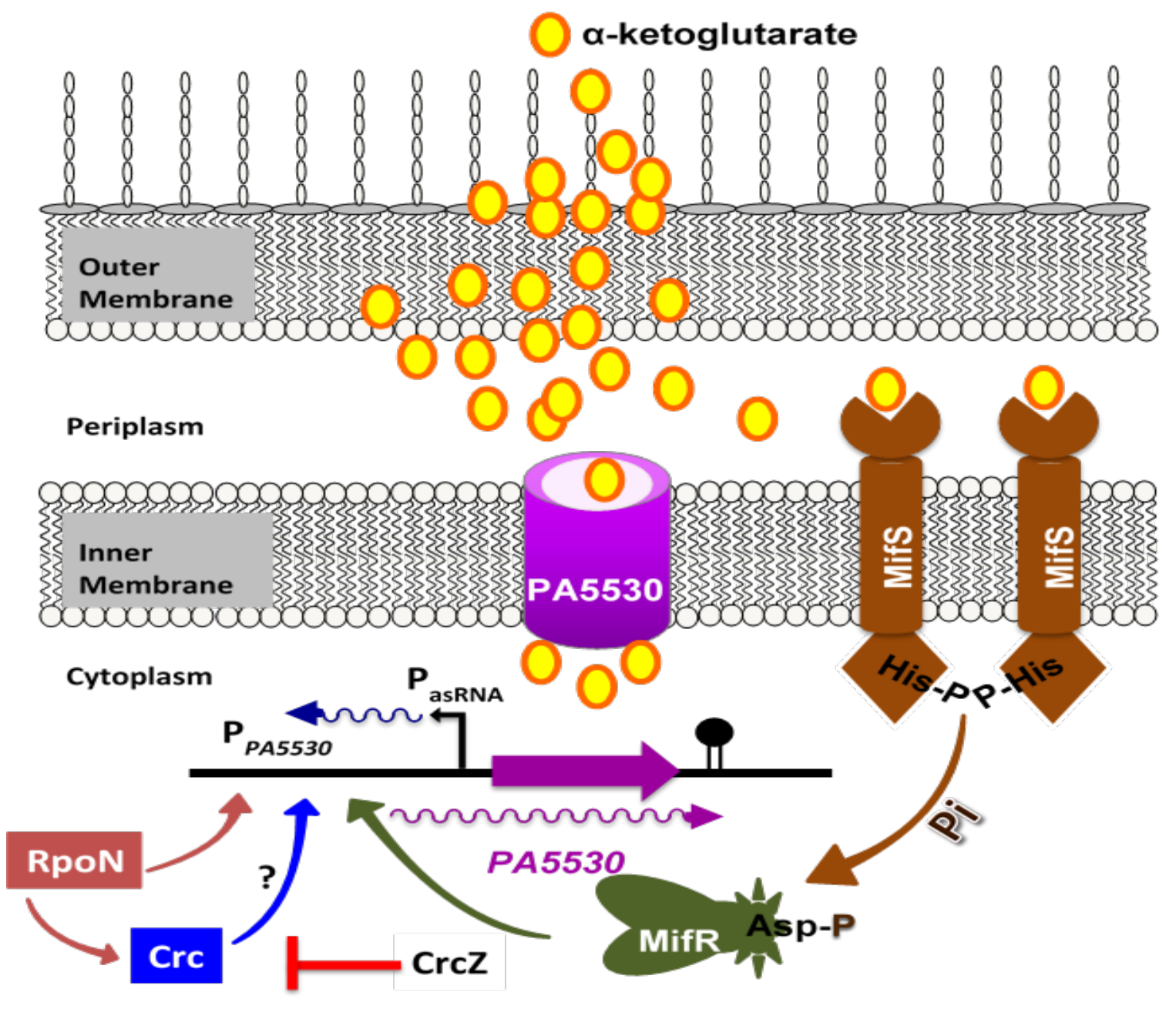

Figure 2.14: Proposed model for $\alpha-K G$ utilization in $P$. aeruginosa. HKMifS senses the extracellular $\alpha-K G$ to undergo phosphorylation. The phosphate is transferred to the RR-MifR. The phosphorylated MifR in coordination with RpoN $\left(\sigma^{54}\right)$ activates the expression of $\alpha-K G$ specific transporter gene PA5530. PA5530 thus enables the influx of $\alpha-K G$ to meet the metabolic and energy demands of the cells. PA5530 promoter ( $\left.P_{P A 5530}\right)$ region has a Crc binding site (Figure 2.13), suggesting that it is under the catabolite repression control by $\mathrm{Crc} / \mathrm{CrcZ}$. The $\mathrm{P}_{P A 5530}$ also shows the presence of another uncharacterized small non-coding asRNA indicating a multilayered and complex regulation of the $\alpha-K G$ transport system. 


\section{Discussion}

$P$. aeruginosa pathogenicity relies significantly on its metabolic flexibility. However, establishment of successful infection and its progression requires more than just meeting nutritional demands. Precision in sensing environmental signals concomitant with a quick and appropriate response is the key to efficient bacterial adaptation and survival. An arsenal of TCSs encoded in its genome has furnished $P$. aeruginosa with a sophisticated capability to regulate diverse metabolic and virulence processes, ensuring its success as a pathogen (Nishijyo et al., 2001, Gooderham \& Hancock, 2009, Winsor et al., 2011). P. aeruginosa genome encodes one of the largest groups of TCS proteins identified in any sequenced bacterial species (Barakat et al., 2009, Winsor et al., 2011). Bacterial TCS's sense and respond to a variety of external cues such as nutrient availability, osmolarity, redox state, temperature, and concentrations of other extracellular molecules (Stock et al., 2000). However, very few TCS signaling molecules have been identified to date. In this study we suggest that the $P$. aeruginosa MifSR TCS exclusively senses $\alpha-K G, a C_{5}$ dicarboxylate and a key component of TCA cycle.

\section{$P$. aeruginosa antibiotic resistance is independent of MifSR TCS}

A common feature of bacterial genomes is a close association between the functionally related genes and their location on the chromosome (Dandekar et al., 1998, Overbeek et al., 1999). Typically, genes encoding functionally related HKs

and RRs are often physically linked and are co-transcribed as an operon (Stock et al., 1989, Chen et al., 2004). Indeed, our in silico analysis (Figure 2.1A and 2.1B) and cDNA amplification (Figure 2.1C) reveled that mifS-mifR genes are co- 
transcribed and form an operon. This also suggests that HK-MifS and RR-MifR are functionally related and work as a TCS pair. In addition, TCS proteins are known to regulate expression of genes in their immediate vicinity (Stock et al., 1989). The mifSR genes are 81 bp upstream of the two-gene poxAB (PA5513-5514) operon. Due to the proximity of mifSR to poxB which encodes for a $\beta$-lactamase, we postulated that mifSR TCS regulates antibiotic resistance. However, our initial results nullified this hypothesis in which comparative MIC's (Data not shown) and qRT-PCR data (Figure 2.2) showed no difference in antibiotic resistance profiles or poxB expression between the wild-type PAO1 and mifSR single and double deletion mutants.

\section{MifSR TCS regulates $P$. aeruginiosa $\alpha-K G$ utilization}

A previous transcriptome study of the wild-type PAO1 and a mifR deletion mutant cultivated under biofilm-specific conditions showed significant alteration in the expression of genes involved in regulating $P$. aeruginosa metabolism, small molecule transport and amino acid biosynthesis (Petrova et al., 2012). The majority of the changes observed in phenotypic microarrays of the mifS and mifR mutant strains cultivated under planktonic conditions were associated with chemical sensitivity and not with metabolism (Figure 2.3B). Only $12-16 \%$ of phenotypic changes were associated with metabolism. This confirms the significant metabolic differences in the rich planktonic versus anaerobic mode of biofilm growth in $P$. aeruginosa (Waite et al., 2006).

Petrova et al. (2012) have also demonstrated that genes involved in energy metabolism, including anaerobic metabolism and fermentative pathways using 
arginine $(\operatorname{arcDABC})$ and pyruvate, were expressed significantly less in $\triangle m i f R$ mutant biofilms as compared to its parent PAO1 (Petrova et al., 2012). Though pyruvate is needed for biofilm formation, it cannot compensate for the loss of mifR (Petrova et al., 2012). Interestingly, the biofilm phenotype associated with the loss of mifR can be complemented by IdhA encoding D-lactate dehydrogenase to wild type levels of biomass accumulation and microcolony formation (Petrova et al., 2012). These findings suggest that MifR somehow regulates expression of $I d h A$, a second gene in a three-gene operon gacS-IdhA-PA0926 (Winsor et al., 2011). Importantly, analyses of the promoters reveal the presence of a shared motif in $P_{\text {mifs }}$ (GATCCGCCGATGTCC) and $P_{P A 5530}($ GATCGGCGGATTTCC) (Figure 2.13) and $P_{\text {gacs }}$ (AATCCGCCGGGCTGC) suggesting a possible coordinate regulation, and that need to be verified.

Our phenotypic microarray analyses and growth experiments suggested that $P$. aeruginosa a-KG utilization requires MifS and MifR (Figure 2.5 and 2.7A). The ability of PAO $\triangle$ mifR, PAO $\Delta$ mifS and PAO $\triangle$ mifSR to grow in the presence of a-KG was restored by in trans expression of mifR and mifSR (Figure 2.7A). Interestingly, the PAO $\triangle$ mifS was complemented by pMifR and pMifSR (Figure 2.5D) but not by pMifS alone. To rule out that gene expression may have been compromised, the mifS gene was cloned downstream of the inducible $\mathrm{P}_{\text {lacUv5 }}$ promoter. Though the expression of stable protein was visible in a protein gel, it failed to complement PAO $\Delta$ mifS mutant (data not shown). This suggests that cisexpression of mifS and mifR is critical for MifS-function. Other researchers have encountered similar problems involving histidine kinases (Yeung et al., 2011). 
Moreover, complementation of the PAO $\Delta$ mifS with pMifR suggests that either phosphorylation is not required or there is a potential crosstalk between MifR and other non-cognate HKs. Alternatively, phosphorylation of MifR can occur through small molecule phosphor-donors, like acetyl phosphate, carbamoyl phosphate and phosphoramidate (Deretic et al., 1992). Such phenomenon is observed with other TCS RRs (Lukat et al., 1992, Wanner \& Wilmes-Riesenberg, 1992, Varughese, 2002). However, this has to be verified.

The $\mathrm{C}_{5}$-dicarboxylate $\alpha-K G$ is an important intermediate in the energygenerating TCA cycle (Figure 2.8) and plays a key role in regulating carbon and nitrogen metabolism (Doucette et al., 2011). Similar to other bacteria (Janausch et al., 2002), TCS's in $P$. aeruginosa have been reported to regulate transport and utilization of TCA cycle intermediates such as succinate, fumarate, malate and citrate (Nishijyo et al., 2001, Valentini et al., 2011). The R. meliloti DctB/DctD system is a well-characterized TCS that controls the transport of TCA cycle $\mathrm{C}_{4^{-}}$ dicarboxylates succinate, fumarate and malate (Janausch et al., 2002). Though $P$. aeruginosa MifS/MifR proteins are homologous to $R$. meliloti DctB/DctD TCS proteins, the mifSR mutants efficiently utilized citrate, succinate, fumarate, malate, oxaloacetate, sucrose and glucose but exclusively failed to grow in the presence of $\alpha-K G$ (Table 2.3). This was further supported by another parallel study that shows that $\alpha-K G$ utilization requires MifR (Lundgren et al., 2014). Thus, the $P$. aeruginosa MifSR TCS is specifically and uniquely involved in $\mathrm{C}_{5}$-dicarboxylate $\alpha$ KG utilization. 


\section{MifSR TCS modulates $P$. aeruginosa $\alpha-K G$ transport}

The inability to utilize $\alpha-K G$ suggested that the mifSR mutants either have a defective $\alpha-K G$ dehydrogenase complex (inability to convert $\alpha-K G$ to succinyl$\mathrm{coA}$, Figure 2.8), or they are deficient in the transport of $\alpha-K G$ into the cell. The former was ruled based upon multiple findings: unchanged expression levels of genes encoding $\alpha-K G$ dehydrogenase, Ipd3 (PA4829) and sucA (PA1585) (Figure 2.10); the ability to use $C_{4}$ and $C_{6}$ dicarboxylates (Table 2.3) and $C 5$ family of amino acids such as arginine, proline, glutamine, and histidine (Table 2.4). The C5 family of amino acids act as biosynthetic precursors of glutamate that ultimately are converted to $\alpha-K G$ by a transamination reaction or through the action of glutamate dehydrogenase (Lehninger et al., 2013). These findings strongly argued that the mifSR mutants were defective in their ability to transport $\alpha-K G$ into the cell.

To date, among the identified carboxylate transporters, the $\mathrm{C}_{4}$-dicarboxylate transporters have been reasonably well characterized. Based on protein sequence similarity analysis, bacterial $\mathrm{C}_{4}$-dicarboxylate transporters are classified into five families, namely, dicarboxylate transport (DctA); dicarboxylate uptake (DcuAB), (DcuC) and (CitT) and the tripartite ATP-independent periplasmic (TRAP) families (Janausch et al., 2002). Amongst these, DctA transporters, a subgroup of the dicarboxylate/amino acid:çation symporter (DAACS) family (Busch \& Saier, 2002, Busch \& Saier, 2004, Saier et al., 2006), are extensively studied and are implicated in the transport of $\mathrm{C}_{4}$-dicarboxylates in Echerischia coli (Davies et al., 1999), Bacillus subtilis (Asai et al., 2000), Rhizobium meliloti (Yarosh et al., 1989, Watson, 1990), Rhizobium leguminosarum (Finan et al., 1981, Ronson et al., 1987) and 
Corynebacterium glutamicum (Youn et al., 2009). As we were trying to identify the MifSR-dependent transporter Lundgren et al., reported that PA5530 is involved in a-KG transport (Lundgren et al., 2014). As predicted, in trans expression of PA5530 was able to restore the ability of mifR, mifS and mifSR mutants to grow in $\alpha-K G$ (Figure 2.5E). This is further confirmed by the increase in PA5530 expression in PAO1 in the presence of $\alpha-K G$ (Figure 2.11A). PA5530 shares no homology with the $P$. aeruginosa $\mathrm{C}_{4}$-dicarboxylate transporter PA1183 (DctA). However, it does have conserved protein domain family PRK10406 implicated in a-KG transport and shares $\sim 70 \%$ homology to E. coli and Erwinia spp. $\alpha-K G$ permease KgtP (Seol \& Shatkin, 1991, Marchler-Bauer et al., 2015). A common feature in the transport of $\mathrm{C}_{4}$-dicarboxylates and other carbon sources in different bacteria is the involvement of TCS mediated regulatory mechanism. Involvement of TCSs, a stimulusresponse coupled mechanism, in the transport of $\mathrm{C}_{5}$-dicarboxylates suggests a more profound role of $\alpha-K G$ as a signaling molecule.

\section{$P$. aeruginosa $\alpha-K G$ transport requires functional $\operatorname{RpoN}\left(\sigma^{54}\right)$}

$P$. aeruginosa RpoN $\left(\sigma^{54}\right)$ is involved in a myriad of functions including expression of virulence factors and nutrient uptake (Potvin et al., 2008). Functional RpoN is reported to be critical for maintaining a carbon-nitrogen balance in Pseudomonads (Kohler et al., 1989, Nishijyo et al., 2001, Cases et al., 2003, Li \& Lu, 2007, Zhang \& Rainey, 2008). Sequence analysis of MifR indicated a requirement of functional RpoN in modulating $P$. aeruginosa a-KG utilization. Our study confirms that $\alpha-K G$ utilization in $P$. aeruginosa PAO1 requires functional RpoN (Table 2.5). This phenotype is not strain-specific as phenotypic microarray 
profiling (BioLOG) of $P$. aeruginosa PA14 rpoN mutant exhibited a similar phenotype, a significant difference in the ability to utilize $\alpha-K G$ as a carbon source as compared to the wild-type PA14 (Behrends et al., 2013). An RpoN-dependent phenotype was also observed with citrate and 4-hydroxyphenylacetate utilization (Behrends et al., 2013). Similarly, utilization of $\mathrm{C}_{4}$-dicarboxylates succinate, fumarate and malate in $R$. meliloti and $P$. aeruginosa also requires the sigma factor RpoN $\left(\sigma^{54}\right)$ (Ronson et al., 1987, Ronson et al., 1987, Valentini et al., 2011).

The need for RpoN $\left(\sigma^{54}\right)$ to utilize $\alpha-K G$ in $P$. aeruginosa can be bypassed by expressing PA5530 encoding for the transporter under a regulatable promoter but not MifS and MifR. Consistent with the need for RpoN $\left(\sigma^{54}\right)$, the promoter for PA5530 has the requisite signature sequences (Figure 2.13). Like most complex RpoN-dependent promoters (Merrick, 1993), the region is long with multiple motifs that include a signature sequence (AAc/uAAc/uAA) for catabolite repression control (Crc) protein, a post-transcriptional inhibitor that binds the mRNA preventing translation (Moreno et al., 2007, Sonnleitner et al., 2009, Browne et al., 2010). Expression of $c r c$ is in-turn regulated by RpoN-dependent non-coding RNA CrcZ (Sonnleitner et al., 2009) whose absence in rpoN mutant can also lead to reduced expression of PA5530. Also, analysis of $P$. aeruginosa PA14 transcripts indicates that the PA5530 promotor is under a small non-coding antisense RNA (asRNA) regulation (Wurtzel et al., 2012). Though the role of Crc, CrcZ and the asRNA in $\alpha-K G$ transport has to be verified experimentally, it suggests an additional layer of regulation superimposed on the need for MifS and MifR on the expression of the $\mathrm{C}_{5}$-dicarboxylate transporter PA5530. 


\section{Conclusion}

In eukaryotic cells, the mitochondria serve as a hub and reservoir of the TCA cycle and its intermediates, respectively. Bacterial pathogens can be highly virulent intruders of the host tissue, causing significant damage leading to cellular aberrations and injury. Mitochondrial dysfunction, a consequence of cell injury, results in efflux of TCA cycle intermediates leading to an increase in their extracellular concentrations (Hebert, 2004). It is known that TCA cycle intermediates $\left(\mathrm{C}_{4}, \mathrm{C}_{5}\right.$, and $\mathrm{C}_{6}$ dicarboxylates $)$ are present at micromolar $(\mu \mathrm{M})$ concentrations in blood that increase with tissues damaged (He et al., 2004, Hebert, 2004). a-KG can also act as a reactive oxygen species scavenger, especially for hydrogen peroxide, protecting both host and pathogen (Long \& Halliwell, 2011). For pathogenic bacteria such as $P$. aeruginosa, efficient uptake of TCA intermediates from the host is crucial for its survival, especially when it is bombarded with host reactive oxygen species, and requires the activity of bacterial carboxylate transport proteins. The transport proteins could be specific for $\mathrm{C}_{4}, \mathrm{C}_{5}$, and $\mathrm{C}_{6}$ intermediates and may use a cognate TCS. This study suggests a complex regulatory cascade in modulating $P$. aeruginosa $\mathrm{C}_{5}$-dicarboxylate, $\alpha-K G$ uptake involving the PA5530 transporter, the MifS/MifR TCS and the sigma factor RpoN (Figure 2.14). It appears that MifS senses the presence of $\alpha-K G$ and signals MifR. The activated MifR in concert with RpoN initiates the transcription of a-KG-specific transporter gene PA5530. Analyses of the published data suggests that the PA5530 promoter is under several layers of regulation including catabolite repression mediated by $\mathrm{Crc} / \mathrm{CrcZ}$ (Sonnleitner et al., 2009) and the small non- 
coding asRNA (Wurtzel et al., 2012). Though the asRNA has been identified (Wurtzel et al., 2012), it has not been characterized. It is not surprising that the PA5530 expression is potentially regulated by $\mathrm{Crc}$, as it would allow control of transporter(s) in response to the presence of carbon sources in the environment. In addition to MifSR (PA5512/PA5511), PA1336/PA1335 have been identified to be homologous to the Rhizobium $\mathrm{C}_{4}$-dicarboxylate transport regulatory DctB/DctD TCS (Stover et al., 2000, Valentini et al., 2011). However, the role of PA1336/PA1335 remains to be elucidated. The $P$. aeruginosa genome also encodes 19 other paralogs of PA5530 dicarboyxlate transporters, most of which have share less than $50 \%$ similarity except for PA0229 (PcaT). PA0229 and PA5530 have $73 \%$ similarity. Future studies will determine if the transporters are preferentially or hierarchically upregulated depending on the carbon source. It is also important to note that much of bacterial physiology, particularly of pathogens such as $P$. aeruginosa remains a mystery. Metabolic versatility, expression of virulence factors and antibiotic resistance together makes $P$. aeruginosa an portentous pathogen. Thus, understanding the physiological cues and regulation would provide a better stratagem to fight the often indomitable infections.

\section{Acknowledgments}

The authors thank the following individual for their intellectual input: Kyle Martins and Jeremy Chambers (Florida International University), Deepak Balasubramanian (Harvard Medical School), Lars Dietrich (Columbia University), and Elaine Newman (Concordia University, Canada). We would like to thank Dr. 
D. Haas from UNIL, Switzerland for kindly providing PA0 $\Delta r p o N$ and PA0 $\Delta r p o N:: r p o N$ 


\section{CHAPTER 3}

Contemporary role for the ancient metabolite $\alpha$-ketoglutarate in regulating Pseudomonas aeruginosa pathogenicity

Gorakh Tatke, Sushmita Mustafi, M. A. Barbieri and Kalai Mathee (Being Submitted) 


\section{Abstract}

Pseudomonas aeruginosa is a Gram-negative, opportunistic pathogen responsible for causing incapacitating infections in individuals with impaired immunity. Successful infection and disease progression relies significantly on the ability of any pathogen to effectively utilize available nutrients that are essential for its growth and survival. Unlike other pathogenic bacteria where glucose is the preferred carbon source, $P$. aeruginosa preferentially utilizes tricarboxylic acid (TCA) cycle intermediates as carbon, nitrogen and energy sources. In $P$. aeruginosa, two-component system (TCS) signaling proteins have played an integral part in regulating the uptake of TCA cycle intermediates, specifically, $\mathrm{C}_{4}$ dicarboxylates such as succinate, fumarate and malate. We recently identified a TCS protein pair PA5512/PA5511 (MifS/MifR) that in- tandem with the sigma factor RpoN $\left(\sigma^{54}\right)$ facilitate the uptake of extracellular $C_{5}$-dicarboxylate $\alpha$-ketoglutarate ( $\alpha-K G$ ) by activating the expression of $P$. aeruginosa $\alpha-K G$-specific permease PA5530 (KgtP). In the present study, we demonstrate that besides KgtP, $P$. aeruginosa genome encodes a second $\alpha-K G$ transporter PA0229 (PcaT) whose expression is independent of the MifSR TCS, suggesting an additional layer of regulation involved in $P$. aeruginosa $\alpha-K G$ uptake. Importantly, in the recent years, $\alpha-K G$ has become the focal point of research because of its newly identified role as a signaling molecule in-addition to its conventional role in metabolism. To date, very little is known of the physiology of $P$. aeruginosa when provided with $\alpha-K G$ as the sole carbon source and their role in pathogenesis. Using PAOAmifS, $\mathrm{PAO} \Delta$ mifR and $\mathrm{PAO} \Delta$ mifSR mutants expressing the $\alpha-K G$ transport protein PcaT 
in trans, we additionally investigated the role of MifSR TCS and $\alpha-K G$ in regulating P. aeruginosa virulence. Multiple virulence phenotypes were tested in the presence of $\alpha-K G$, citrate and/or succinate, as a sole carbon source. In comparison to the wild-type PAO1 strain, mifSR deletion strains exhibited differential regulation of biofilm formation, pyocyanin and pyoverdine production, motility, and cell cytotoxicity in the presence of $\alpha-K G$. Subsequently, mifSR mutant derivatives exhibited no discernible phenotypes compared to the parent PAO1 when $\alpha-K G$ was replaced with citrate and/or succinate, as the sole carbon source. Thus, MifSR TCS regulates the expression of $P$. aeruginosa's key virulence determinants in $\alpha$ KG-dependent manner. Simultaneous regulation of multiple mechanisms involved in $P$. aeruginosa pathogenesis suggests a complex mechanism of MifSR action. Understanding the physiological cues and regulation would provide a better stratagem to fight the often indomitable $P$. aeruginosa infections.

\section{Introduction}

Despite continuous efforts in the development of new antimicrobial drugs, bacterial infections are frequently associated with high morbidity and mortality rates. In particular, infections caused by the multidrug resistant bacteria, including, but not restricted to the so called "ESKAPE" pathogens (Enterococcus faecium, $\underline{S}$ taphylococcus aureus, $\underline{K}$ lebsiella species, $\underline{A}$ cinetobacter baumannii,

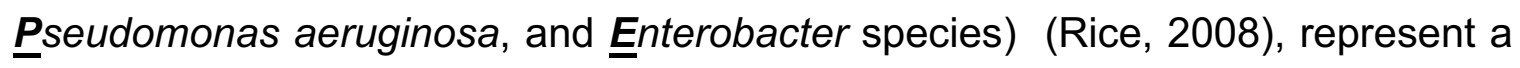
major public health concern (Tommasi et al., 2015) and economic burden worldwide (Boucher et al., 2009). Amongst these organisms, P. aeruginosa is 
frequently implicated in causing injurious infections (Rice, 2008), and has gained significant attention over the years as one of the most portentous pathogen.

$P$. aeruginosa is a Gram-negative, $Y$-proteobacteria that is widely distributed in nature (Green et al., 1974). It is well-known for its nutritional and ecological resilience, which enables it to endure and thrive in diverse environmental settings, both natural and artificial (Ramos, 2004). Although, $P$. aeruginosa is a multi-host pathogen infecting insects (Bulla, 1975), nematodes (Mahajan-Miklos et al., 1999), plants (Sing \& Schroth, 1977, Rahme et al., 1995) and mammals (Stevens et al., 1994), it is the epitome of opportunistic human infections (Ramos, 2004). It is one of the most feared nosocomial pathogen, accounting for $10 \%$ of all the hospital acquired infections (Hancock \& Speert, 2000 , Sievert et al., 2013). P. aeruginosa can colonize different tissue types leading to multifactorial clinical and pathological manifestations including pneumonia (Driscoll et al., 2007), urinary tract infection (Driscoll et al., 2007), gastrointestinal infection (Markou \& Apidianakis, 2014), chronic suppurative otitis media (Yeo et al., 2007, Mittal et al., 2015), endocarditis (Fish et al., 1937, Sandre \& Shafran, 1996), osteomyelitis (Carek et al., 2001), keratitis (Dart \& Seal, 1988), bacteremia (Kerby, 1947) and soft-tissue infection (Moet et al., 2007). The largest cohorts of $P$. aeruginosa infected patients are the ones with AIDS, severe burn wounds, cystic fibrosis (CF), chronic obstructive pulmonary disease (COPD), diabetes, cardiovascular disease, cancer and those with acquired or primitive immunological abnormalities (Manfredi et al., 2000, Ramos, 2004, Driscoll et al., 2007, Vento et al., 2008, Valderrey et al., 2010). Also, beyond high infection rate, P. aeruginosa 
has the highest case fatality rate of all Gram-negative infections (Aliaga et al., 2002). P. aeruginosa is extensively armed with a set of multi-determinant virulence factors, both cell surface associated (flagella, pili, LPS) and secretory (proteases, exotoxins, phenazines, haemolysins), which modulates its pathogenic potential. In addition to its ability to dodge host immunity, a major setback in effectively treating $P$. aeruginosa infections is its extraordinary intrinsic and acquired resistance to a gamut of clinically critical antibiotics (Breidenstein et al., 2011). Expression of diverse virulence factors and antibiotic resistance together, makes $P$. aeruginosa an indomitable pathogen.

Metabolic versatility is the key to bacterial pathogenesis and disease progression. In order to survive and multiply in the host systems, bacterial pathogens have developed mechanisms to regulate their metabolism according to the nutrient composition and availability (Silby et al., 2011). Carbon and nitrogen are the two major nutrients required by all living organisms to perform fundamental cellular activities. P. aeruginosa uses an eclectic range of organic compounds including carbohydrates, amino acids, fatty acids, mono- and polyalcohols, di- and tri-carboxylic acids as carbon, nitrogen and energy source (Ramos, 2004). Although efficiently metabolized via the Entner-Doudoroff pathway, sugars are less preferred carbon source (Entner \& Doudoroff, 1952, Lessie \& Phibbs, 1984). Instead, $P$. aeruginosa has a predilection for tricarboxylic acid (TCA) cycle intermediates, specifically, $\mathrm{C}_{4}$-dicarboxylates of the TCA cycle such as malate, fumarate and succinate (Liu, 1952, Collier et al., 1996). 
The TCA cycle, classical (Krebs, 1940) or the modified form (Romano \& Conway, 1996, Hugler et al., 2005, Zhang \& Bryant, 2011), is one of the most important central metabolic pathway used by all organisms to generate energy. Essentially, the TCA cycle plays a dual role in cell metabolism: 1) it produces carbon dioxide and reduced electron carriers $\left(\mathrm{NADH}\right.$ and $\left.\mathrm{FADH} \mathrm{H}_{2}\right)$ by oxidation of acetyl-CoA to generate energy (catabolism), and 2) provides cells with precursor metabolites as building blocks for the synthesis of carbohydrates, fatty acids, nucleic acids and amino acids (anabolism) (Owen et al., 2002). Because of its amphibolic nature and ubiquity, it is not surprising that different organisms use and modulate TCA cycle according to their metabolic requirements, thus giving rise to a considerable diversity in its way of operating. Moreover, it is becoming evident that the TCA cycle is embedded in a more complex network of metabolic pathways, and its intermediates, beyond their canonical function play a profound role as intraand extracellular signals (Benit et al., 2014, Huergo \& Dixon, 2015, Haas et al., 2016).

TCA cycle intermediates are organic acids, that based on the number of carbon atoms are classified into three groups $\mathrm{C}_{4}$-dicarboxylates (succinate, fumarate, malate and oxaloacetate), $C_{5}$-dicarboxylate ( $\alpha$-Ketoglutarate $(\alpha-K G)$ ) and $\mathrm{C}_{6}$-tricarboxylates (citrate and iso-citrate) (Krebs, 1940). Amongst these, the $\mathrm{C}_{5}$-dicarboxylate $\alpha-K G$ has gained significant attention because of its newly established role as a signaling molecule in-addition to its conventional role in metabolism. There is mounting evidence that corroborates the role of $\alpha-K G$ as a signaling molecule in all three domains of life, archaea (Dodsworth et al., 2005), 
bacteria (Ninfa \& Jiang, 2005) and eukaryota (Lancien et al., 2000, He et al., 2004). Indeed, it is now known that $\alpha-K G$ plays a key role in many signaling pathways including, but not limited to, regulation of carbon/nitrogen metabolism (Doucette et al., 2011), cyclic AMP (cAMP) synthesis (Rabinowitz \& Silhavy, 2013), paracrine mediator in regulating renal acid-base balance (Tokonami et al., 2013) and in epigenetic regulation mediated through histone modification (Letouze et al., 2013, Carey et al., 2015) and a-KG-dependent oxygenases (Loenarz \& Schofield, 2008). In bacteria, intracellular levels of $\alpha-K G$ acts as a key indicator of the cellular $\mathrm{C} / \mathrm{N}$ status (Doucette et al., 2011). $\alpha-K G$ provides the major carbon skeleton for nitrogen assimilation pathways (Commichau et al., 2006). High levels of intracellular $\alpha-K G$ signals nitrogen starvation, thereby activating the transcription of nitrogen assimilation genes (Leigh \& Dodsworth, 2007). It is now well established that $\alpha-K G$ directly regulates nitrogen metabolism by binding and modulating the activity of either the nitrogen regulatory PII protein GInB in E. coli (Ninfa \& Jiang, 2005) and/or the transcription factor NtcA in cyanobacteria (Tanigawa et al., 2002, Vazquez-Bermudez et al., 2002). Signal perception mechanisms mediated by $\alpha-K G$, nitrogen regulatory PII and NtcA proteins has been studied and reviewed extensively (Forchhammer, 2008, Forchhammer, 2010, Huergo et al., 2013, Huergo \& Dixon, 2015). Furthermore, endogenous $\alpha-$ $\mathrm{KG}$ acts as a reactive oxygen species scavenger, particularly of hydrogen peroxide thus acting as a protectant against oxidative damage (Mailloux et al., 2009, Lemire et al., 2010) and cyanide poisoning (Kunz et al., 1998). Recent studies have reported the use of exogenous $\alpha-K G$ as a preferred carbon source during the 
course of infection by pathogenic bacteria (Guo et al., 2012, Cai et al., 2013). This together suggests a more intricate and intense role of $\alpha-K G$ in regulating bacterial physiology and metabolism.

Sensing the available nutrient is a prerequisite for bacterial growth and virulence. Bacteria have evolved various host-adapted nutrient acquisition strategies to modulate the uptake systems. One canonical mechanism by which most bacteria sense and respond to the available nutrient is the two-component systems (TCSs). TCSs are made of two signal transduction proteins, a membranebound histidine sensor kinase (HK) and a cytoplasmic response regulator (RR), usually a DNA binding protein (Gross et al., 1989). In response to stimuli, the sensor autophosporylates at a conserved histidine residue at the C-terminus, and subsequently the phosphate is transferred to an aspartate residue at the $\mathrm{N}$ terminus of the RR (Stock et al., 1989, Stock et al., 2000). The phosphorylated RR then modulates the expression of various genes required to bring about the appropriate response (Bourret et al., 1989). Given the importance of TCA cycle intermediates as a preferred carbon source in numerous bacteria (Finan et al., 1983, Collier et al., 1996, Tang et al., 2005), it is not surprising that the TCSs are involved in regulating their uptake. In both, Gram-negative and Gram-positive bacteria TCS proteins have been implicated in regulating the transport and utilization of TCA cycle intermediates, particularly the $\mathrm{C}_{4}$-dicarboxylates (succinate, fumarate and malate) and the $\mathrm{C}_{6}$-tricarboxylate (citrate) (Asai et al., 2000, Janausch et al., 2002). However, not much is known about the assimilation of the $\mathrm{C}_{5}$-dicarboxylate $\alpha-K G$. 
The TCS proteins responsive to extracellular $\alpha-K G$ has been identified in limited number of bacterial species, including Escherichia coli (Cai et al., 2013), Rhizobium tropici (Batista et al., 2009) and most recently in P. aeruginosa (Tatke et al., 2015). In P. aeruginosa, a TCS protein pair PA5512/PA5511 (MifS/MifR) together with the sigma factor $\mathrm{RpoN}\left(\sigma^{54}\right)$ regulates the transport of extracellular $\alpha-K G$ by activating the expression of the $\alpha-K G-s p e c i f i c$ permease PA5530, a Major Facilitator Superfamily (MFS) of secondary active transporter protein (Lundgren et al., 2014, Tatke et al., 2015). P. aeruginosa MifS/MifR pair is homologous to the Rhizobium meliloti $\mathrm{C}_{4}$-dicarboxylate transport regulatory DctB/DctD TCS (Winsor et al., 2011). No role of $P$. aeruginosa MifS and MifR in the utilization of TCA cycle $\mathrm{C}_{4}-\mathrm{C}_{6}$ di and tricarboxylates (succinate, fumarate, malate, oxaloacetate and citrate) has been established (Tatke et al., 2015). Thus, P. aeruginosa MifSR TCS is specifically and uniquely involved in $\alpha-K G$ utilization.

Previously, the MifR protein has been identified to be essential for regulating P. aeruginosa biofilm formation (Petrova \& Sauer, 2009) and pyruvate fermentation (Petrova et al., 2012). Additionally, in P. aeruginosa, nutritional cues such as individual carbon and nitrogen sources have been known to influence several clinically relevant virulence phenotypes such as motility, biofilm development, quorum sensing and pigment production (Kohler et al., 2000, Klausen et al., 2003(a), Palmer et al., 2005, Shrout et al., 2006, Palmer et al., 2007). To date, very little is known about the physiology of $P$. aeruginosa when provided with $\alpha-K G$ as the sole carbon source. Here we show that, MifS/MifR TCS and the $\mathrm{C}_{5}$-dicarboxylate $\alpha$-KG plays a perspicacious role in regulating $P$. 
aeruginosa pathogenicity by modulating its key virulence traits, including, motility, pigment production, biofilm formation and cell cytotoxicity. This study is one of the first to report a novel role of the regulatory mechanism exerted by MifSR TCS and significantly highlights the potential role of $\alpha-K G$ as a signaling molecule in $P$. aeruginosa.

\section{Materials \& Methods}

\section{Bacterial strains, media and growth conditions}

The bacterial strains and plasmids used in this study are listed in Table 3.1. Briefly, strains of Pseudomonas aeruginosa and Escherichia coli were routinely cultured in Luria Bertani (LB) broth ( $5 \mathrm{~g}$ tryptone, $10 \mathrm{~g}$ sodium chloride, and $5 \mathrm{~g}$ yeast extract per liter) or agar (LB broth with 1.5\% agar) (Difco, NJ, USA) or in M9 minimal Media $\left(64 \mathrm{~g} \mathrm{Na}_{2} \mathrm{HPO}_{4}-7 \mathrm{H}_{2} \mathrm{O}, 15 \mathrm{~g} \mathrm{KH}_{2} \mathrm{PO}_{4}, 2.5 \mathrm{~g} \mathrm{NaCl}, 5.0 \mathrm{~g} \mathrm{NH}_{4} \mathrm{Cl}, 20\right.$ $\mathrm{mM} \mathrm{MgSO}{ }_{4}, 1 \mathrm{mM} \mathrm{CaCl}_{2}$ per liter) (Sambrook \& Russell, 2001) at $37^{\circ} \mathrm{C}$, unless specified otherwise. $P$. aeruginosa competent cells were prepared using the sucrose $(300 \mathrm{mM})$ method as previously described (Choi et al., 2006). Growth curves, complementation studies and phenotypic assays were performed in M9 minimal media supplemented with TCA cycle intermediates, $\alpha-K G$, succinate and/or citrate (at $30 \mathrm{mM}$ each), as described previously (Tatke et al., 2015). For selection and maintenance of plasmids, antibiotics when used, were at the following specified concentrations, unless specified otherwise: E. coli: ampicillin (Ap) $100 \mu \mathrm{g} / \mathrm{ml}$, gentamycin $15 \mu \mathrm{g} / \mathrm{ml}$, kanamycin $(\mathrm{Km}) 20 \mu \mathrm{g} / \mathrm{ml}, P$. aeruginosa: $\mathrm{Gm} 75 \mu \mathrm{g} / \mathrm{ml}$. 


\section{Cell culture and media}

A549, a human lung adenocarcinoma epithelial cell line was provided by Dr. Rahul Mittal (University of Miami, Miller School of medicine, Miami, FL). The J774 E-clone, a murine macrophage cell line, also a generous gift from Dr. Philip Stahl (Washington University Medical school, St Louis, MO) and the human epithelial adenocarcinoma cell line was obtained from ATCC (ATCC ${ }^{\circledR}$ CCL-2 ${ }^{\mathrm{TM}}$ ). J774 E-clone cell lines were maintained under a $5 \% \mathrm{CO}_{2}$ atmosphere in Dubelco's Minimum Essential Medium (DMEM), supplemented with 10\% heat-inactivated fetal calf serum (FCS), $2 \mathrm{mM} \mathrm{L-glutamine,} 100$ Units $/ \mathrm{mL}$ penicillin and $100 \mu \mathrm{g} / \mathrm{mL}$ of streptomycin. A549 cells were grown in Roswell Park Memorial Institute Medium (RPMI) 1640 (Life Technologies, Carlsbad, CA, USA) supplemented with 10\% fetal calf serum (FCS), 24 mM 4-(2-hydroxyethyl)-1-piperazineethanesulfonic acid (HEPES), $2 \mathrm{mM} \mathrm{L-glutamine,} 100 \mathrm{Units} / \mathrm{mL}$ penicillin, $100 \mu \mathrm{g} / \mathrm{mL}$ of streptomycin and incubated at $37^{\circ} \mathrm{C}$ in $5 \% \mathrm{CO}_{2}$. HeLa cells were cultured in DMEM supplemented with $10 \%$ FCS and $2 \mathrm{mM} \mathrm{L-glutamine}$ and $1 \%$ non-essential amino acids in presence of $5 \% \mathrm{CO}_{2}$ at $37^{\circ} \mathrm{C}$. All chemicals and reagents were purchased from Sigma-Aldrich (St. Louis, MO), unless otherwise indicated.

\section{Genetic manipulations}

Genetic manipulations were carried out as previously described using standard techniques (Green et al., 2012). Polymerase Chain Reaction (PCR) amplification was performed using the AccuPrime high-fidelity Taq DNA Polymerase (Invitrogen, Life Technologies, Carlsbard, CA, USA). Primers for PCR and sequencing were synthesized by Integrated DNA Technologies, Inc. 
(Coralville, IA, USA) and are listed in Table 3.2. DNA purification and plasmid DNA isolation was carried out using nucleic acid purification kits (Promega, Madison, WI, USA) and PureLink Hipure Plasmid Miniprep Kit (Invitrogen, Life Technologies, Carlsbard, CA, USA). Wizard® SV Gel and PCR Clean-Up System kits (Promega, Madison, WI, USA) were used for PCR product and agarose gel fragment purification. RNA extraction and cDNA synthesis was performed using RNeasy Mini Kit (Qiagen Inc. Venio, Limburg, Netherlands) and SuperScript III First-Strand Synthesis System (Invitrogen, Life Technologies, Carlsbad, CA, USA). Restriction enzymes were from New England Biolabs (Ipswich, MA, USA), and DNA sequencing was carried out at Florida International University (FIU) DNA core and at GENEWIZ Inc. (South Plainfield, NJ, USA). All other chemicals were purchased from Sigma-Aldrich (St. Louis, MO, USA), Amresco (Solon, OH, USA) and TheromoFisher Scientific (Waltham, MA, USA), unless otherwise specified.

\section{PCR amplification and cloning of pcaT}

DNA fragments from PAO1 with $p c a T(\sim 1.3 \mathrm{~kb})$ and $P A 0703(\sim 1.3 \mathrm{~kb})$ were PCR amplified using primer pairs GDT_pcaTF1-GDT_pcaTR1 (Table 3.2), respectively. The primers are designed such that the ORF is contiguous to a strong ribosome binding site (AGGAGA), to ensure expression of the genes (Lehninger et al., 2013). The PCR amplified pcaT was cloned directly into a broad host range pPSV37-Gm (Lee et al., 2010) plasmid as a Nhel-Sacl fragments, downstream of an inducible $\mathrm{P}_{\text {lacUv5 }}$ promoter to generate plasmids pGDT007, respectively. The fidelity of the PCR product was confirmed by sequencing using the primers 
GDT_p37_SeqF-R, pcaT_seqF-R and PA0703_seqF-R (Table 3.2). Henceforth, this plasmid is referred to as pPcaT, respectively.

The expression plasmid pPcaT was subsequently introduced by electroporation (Choi et al., 2006) into PAO1 and its isogenic mutants PAO $\Delta$ mifS, $\mathrm{PAO} \Delta$ mifR, $\mathrm{PAO} \Delta$ mifSR and $\mathrm{PAO} \Delta r p o N$.

\section{Growth curves}

Growth curves were performed as described previously (Tatke et al., 2015). Briefly, $P$. aeruginosa wild type PAO1 and its isogenic mutant derivatives were grown overnight in LB broth with or without antibiotics at $37^{\circ} \mathrm{C}$. After overnight growth, spent and residual media was removed by centrifugation and the cultures were washed with sterile $0.85 \% \mathrm{NaCl}(\mathrm{wt} / \mathrm{vol})$ solution. Cultures were then diluted in fresh M9 minimal media supplemented with TCA cycle intermediates including $\alpha-$ $\mathrm{KG}$, succinate and/or citrate (at $30 \mathrm{mM}$ each) as a sole carbon source to obtain equal optical densities $\left(\mathrm{OD}_{600}\right)$ of 0.025 . Growth was monitored in $48-96$ well plates (Corning-Falcon, NY, USA) by determining absorbance at $600 \mathrm{~nm}$ using BioTek Synergy HT plate reader for $14-18 \mathrm{~h}$ at $37^{\circ} \mathrm{C}$. The experiments were performed multiple times in triplicates.

\section{Motility assays}

Motility assays were examined on $0.3 \%, 0.6 \%$ and $1 \%(\mathrm{wt} / \mathrm{vol}) \mathrm{M} 9$ minimal media agar plates as a described previously (Deziel et al., 2001). All cultures were grown overnight in LB media at $37^{\circ} \mathrm{C}$. Overnight cultures were washed with sterile $0.85 \% \mathrm{NaCl}(\mathrm{wt} / \mathrm{vol})$ solution and diluted to $\mathrm{OD}_{600}$ of 1 . Swimming and swarming motilities were evaluated by spotting $1-2 \mu \mathrm{l}$ cultures onto $0.3 \%$ and $0.6 \%(\mathrm{wt} / \mathrm{vol})$ 
M9 minimal media agar plates with $\alpha-K G$ or citrate (at $30 \mathrm{mM}$ each) as a sole carbon source. Twitching motility was evaluated by stab inoculating $\mathrm{OD}_{600}$ normalized cultures onto $1 \%(\mathrm{wt} / \mathrm{vol}) \mathrm{M} 9$ minimal media agar plates with $\alpha-\mathrm{KG}$ or citrate (at $30 \mathrm{mM}$ each) as a sole carbon source. Plates were incubated at $37^{\circ} \mathrm{C}$ and motility patterns were assessed 24 to $48 \mathrm{~h}$ post-incubation. Diameters of the swim or twitch zones were measured in millimeter $(\mathrm{mm})$ and swarming was assessed by determining the surface coverage on agar plates post incubation. Each form of motility was assessed multiple times with three replicates for each mutant.

\section{Rhamnolipid assay}

P. aeruginosa wildtype PAO1, PAO1(pPcaT), PAO $\Delta$ mifS(pPcaT), $\mathrm{PAO} \Delta \operatorname{mifR}(\mathrm{pPcaT})$ and $\mathrm{PAO} \Delta \operatorname{mifSR}(\mathrm{pPcaT})$ strains were assayed for rhamnolipid production using the cetyltrimethylammonium bromide (CTAB) methylene blue agar plate assay as described previously (Siegmund \& Wagner, 1991). Briefly, all cultures were grown overnight at $37^{\circ} \mathrm{C}$ in LB broth. Spent and residual media from the overnight culture was removed by centrifugation, and the cultures were washed with sterile $0.85 \% \mathrm{NaCl}(\mathrm{wt} / \mathrm{vol})$ solution. Rhamnolipd production was evaluated by spot inoculating $\mathrm{OD}_{600}$ normalized cultures on $\mathrm{M9}$ minimal media supplemented with $30 \mathrm{mM}$ a-KG (unless otherwise specified), $0.0005 \%$ (wt/vol) methylene blue and $0.02 \%(\mathrm{wt} / \mathrm{vol}) \mathrm{CTAB}$. Plates were incubated at $37^{\circ} \mathrm{C}$ for $24 \mathrm{~h}$ followed by incubation at room temperature for at least $48 \mathrm{~h}$ until a blue halo appeared around the colonies, indicating rhamnolipid production (Siegmund \& Wagner, 1991). The 
diameter of the blue halo (in millimeter $(\mathrm{mm})$ ) around the colonies was measured as a mark of rhamnolipid production.

\section{Pyocyanin production}

Pyocyanin produced was measured by extracting the pigment from culture supernatants using the chloroform-hydrochloric acid $(\mathrm{HCL})$ method as described previously (Essar et al., 1990). Briefly, 5-ml culture supernatant from the stationary-phase cultures $(\sim 18 \mathrm{~h})$ grown in M9 minimal media supplemented with TCA cycle intermediates including $\alpha-K G$, citrate and succinate (30 mM each) individually as a sole carbon source was mixed with $3 \mathrm{ml}$ chloroform to extract pyocyanin into the organic phase. Pyocyanin from the organic phase was then reextracted into $1 \mathrm{ml}$ of $0.2 \mathrm{~N} \mathrm{HCl}$. The resulting pink color formation, an indication of the presence of pyocyanin was read at $520 \mathrm{~nm}$. The concentration is expressed as $\mu \mathrm{g}$ of pyocyanin produced per $\mathrm{ml}$ of culture $(\mu \mathrm{g} / \mathrm{ml})$, by multiplying the optical density $\mathrm{OD}_{520}$ by 17.072 (Essar et al., 1990). Absorption spectra analysis (250 nm to $800 \mathrm{~nm}$ ) to detect pyocyanin from the cell free culture supernatants, was performed using the Beckman Coulter Inc., DU 800 spectrophotometer.

\section{Pyoverdine production}

To measure pyoverdine production, cells were grown overnight at $37^{\circ} \mathrm{C}$ in M9 minimal media supplemented with $\alpha-K G$, succinate and/or citrate, individually (at $30 \mathrm{mM}$ each) as a sole carbon source. Extracellular pyoverdine from the culture supernatant was read at $405 \mathrm{~nm}$ and normalized to the initial culture density $\left(O D_{600}\right)$. Pyoverdine levels were expressed as a ratio of $\mathrm{OD}_{405} / \mathrm{OD}_{600}$ (Shen et al., 2002). Absorption spectra analysis (250 $\mathrm{nm}$ to $800 \mathrm{~nm}$ ) to detect pyoverdine from 
the cell free culture supernatants, was performed using the Beckman Coulter Inc., DU 800 spectrophotometer.

\section{Cytotoxicity assay}

P. aeruginosa wild-type PAO1 and its isogenic PAO $\Delta$ mifR, PAO $\Delta$ mifS, PAO $\Delta$ mifSR, mutant strains and its derivatives were used for the cytotoxicity assay. Their cytotoxicity was assessed using a human lung adenocarcinoma epithelial cell line (A549), a murine macrophage cell line (J774 E-clone) and HeLa cells. Bacterial cytotoxicity was quantified by measuring the lactate dehydrogenase (LDH) enzyme activity from the culture supernatant post $24 \mathrm{~h}$ infection with $P$. aeruginosa strains (Hauser \& Engel, 1999). Briefly, monolayers of J774 E-clone, A594 and HeLa cells $\left(10^{6}\right.$ cells $\left./ \mathrm{mL}\right)$ were incubated with bacterial suspension containing $10^{8} \mathrm{CFU} / \mathrm{mL}$ of bacteria. Overnight bacterial cells were subcultured into $\mathrm{M} 9$ minimal media with $\alpha-K G(30 \mathrm{mM})$ to a final $\mathrm{OD}_{600 \mathrm{~nm}}$ of 0.1 . The infection was synchronized by centrifugation for 5 minutes at $1000 \times g$ and incubated for 24 hours at $37^{\circ} \mathrm{C}$ in $5 \% \mathrm{CO}_{2}$. Cell-free culture supernatant were then collected and the amount of lactate dehydrogenase (LDH) enzyme activity was assayed in a 96-well plate using the Sigma kit as per manufacturer's instructions (Sigma Aldrich, St. Louis, MO). Triplicate wells were used for each test, and all tests were repeated at least three times.

\section{Biofilm formation}

Biofilm formation was assessed on 96-well polystyrene flat-bottomed (Costar, Corning NY) and/or on non-tissue culture-treated 96-well polyvinyl chloride (PVC) U-bottom (Falcon) microtiter plates, as described previously 
(Friedman \& Kolter, 2004, O'Toole, 2011). Briefly, PAO $\Delta$ mifS(pPcaT), $\mathrm{PAO} \Delta \operatorname{mifR}(\mathrm{pPcaT}), \mathrm{PAO} \Delta \operatorname{mifSR}(\mathrm{pPcaT}), \mathrm{PAO} 1$ (pPcaT) as well as the control strains PAO1(wildtype), PAOAretS (positive control, hyperbiofilm producer) and $\mathrm{PAO} \Delta p e / \Delta p s / \Delta a l g D$ (negative control, no biofilm producer) were grown in LB broth overnight at $37^{\circ} \mathrm{C}$. Cultures were then diluted to an $\mathrm{OD}_{600}$ of 0.0025 in $\mathrm{M} 9$ minimal media supplemented with $\alpha-K G$, succinate or citrate individually (at $30 \mathrm{mM}$ each). The plates were incubated under static condition at room temperature for 24,48 and 72 hours. At each time point, the culture was decanted and the plates were washed by gently submerging them in a small water tub in order to remove the unattached cells, and the plates were dried for 10-15 minutes at room temperature. To stain the biofilm, $0.1 \%$ solution of crystal violet (St. Louis, MO, USA) in water was added to each well $(200 \mu \mathrm{L})$. After 20 -minute incubation at room temperature, crystal violet was decanted, and the plates were washed 7 to 8 times in a small water tub. Plates were then inverted and left to dry overnight. Quantification of the attached and stained cells was done after solubilization of the dye with absolute ethanol $(200 \mu \mathrm{L})$. The optical density of the solution reflecting the relative biofilm formation was measured at $590 \mathrm{~nm}$ using BioTek Synergy HT (Winooski, VT, USA) and expressed as relative biofilm formation $\left(\mathrm{OD}_{590}\right.$ mutant / $\mathrm{OD}_{590}$ wildtype) (Ueda \& Wood, 2009). The experiments were performed multiple times in triplicates.

\section{RNA isolation and cDNA synthesis}

P. aeruginosa wild-type PAO1 and its isogenic PAO $\Delta$ mifR, PAO $\Delta$ mifS, PAO $\triangle$ mifSR, PAO $\triangle r p o N$ mutant strains and its derivatives were used for RNA extraction. Total RNA was extracted from cells grown to mid-exponential phase 
with or without $\alpha-K G$ (at $30 \mathrm{mM}$ ) treatment. Briefly, cultures were grown overnight in LB broth at $37^{\circ} \mathrm{C}$. Post incubation overnight cultures were washed with sterile $0.85 \%$ saline solution to remove spent media and were subcultured at $37^{\circ} \mathrm{C}, 200$ rpm in LB media. LB media was used as a preferred nutrient source for initial growth of cultures since PAO $\Delta$ mifR, PAO $\Delta$ mifS, PAO $\Delta$ mifSR and PAO $\Delta r p o N$ mutants exhibit growth defects in the presence of $\alpha-K G$ alone. When the cells reached an $\mathrm{OD}_{600}$ of $0.6-0.7$, all the cultures were treated with $30 \mathrm{mM} \alpha-\mathrm{KG}$ for 2 h. Post treatment, the RNA was stabilized by adding phenol-ethanol mixture (Brencic et al., 2009). The stabilized RNA was then isolated using RNeasy Mini Kit (Qiagen Inc. Venio, Limburg, Netherlands) as per manufacturer's protocol. Residual genomic DNA contamination was removed using RQ1 RNase-free DNase (Promega, Madison, WI, USA) and RNA was repurified using RNeasy Mini Kit (Qiagen Inc. Venio, Limburg, Netherlands). RNA quality was preliminarily checked on a denaturing agarose gel (NorthernMax Gly, Ambion) followed by analysis on the Agilent Bioanalyzer (Agilent Technologies, Santa Clara, CA, USA). Samples with RNA Integrity Numbers of 8.0 or above were subsequently used for cDNA synthesis. cDNA was synthesized by annealing NS5 random primers to total purified RNA and subsequent extension was carried out using SuperScript III reverse transcriptase, as per the manufacturers protocol (Invitrogen, Life Technologies, Carlsbard, CA, USA).

\section{Quantitative real-time PCR}

$P$. aeruginosa genes encoding $\alpha-K G$ transporter proteins ( $p c a T$ and PA5530 (kgtP)) and pyocyanin synthesis (phzA1, phzB1, phzA2, phzM and phzS) 
were amplified (qRT-PCR) under $\alpha-K G$ induced condition at $30 \mathrm{mM}$, using the $A B I$ 7500 (Applied Biosystems, Life Technologies, Carlsbard, CA, USA) cycler with Power SYBR Green PCR MasterMix with ROX (Applied Biosystems, Life Technologies, Carlsbard, CA, USA). The gene-specific primers used to quantitate the expression levels of the transcript are listed in Table 3.2. Ten nanograms of cDNA was used per reaction well in the qRT-PCR assays. The cycling conditions used were $95^{\circ} \mathrm{C} / 2$ minutes (holding); 40 cycles of $95^{\circ} \mathrm{C} / 15 \mathrm{sec}, 60^{\circ} \mathrm{C} / 1 \mathrm{~min}$ (cycling); $95^{\circ} \mathrm{C} / 15 \mathrm{sec}, 60^{\circ} \mathrm{C} / 1 \mathrm{~min}, 95^{\circ} \mathrm{C} / 15 \mathrm{sec}\left(0.6^{\circ} \mathrm{C}\right.$ ramp) (melt curve). The reading was normalized to $P$. aeruginosa $c / p X(P A 1802)$, whose expression was determined to remain constant between the samples and conditions tested. The qRT-PCR assays for each gene was repeated twice in triplicate, and the mean value of the quantification was calculated. Melting curves were determined to ensure primer specificity.

\section{Bioinformatic and statistical analyses}

Sequence analyses and multiple sequence alignments were generated using ClustalW2 http://www.ebi.ac.uk/Tools/msa/clustalw2/ and www.pseudomonas.com (Winsor et al., 2011). Student's $t$-test or Analysis of Variance (ANOVA) with post-hoc testing, when applicable, was used to analyze statistical differences. The $t$-test and ANOVA were performed using GraphPad and IBM SPSS statistics 22.0 statistical analysis software. A $p$-value of $<0.05$ was considered statistically significant. 
Table 3.1: Strains and plasmids used in this study.

\begin{tabular}{|c|c|c|c|}
\hline Strain ID & $\begin{array}{l}\text { Strain/Plasmid } \\
\text { Background }\end{array}$ & Relevant characteristics & Source \\
\hline \multicolumn{4}{|c|}{ Escherichia coli } \\
\hline $\mathrm{DH} 5 \alpha$ & E. coli & $\begin{array}{l}\mathrm{F}^{-} \Phi 80 \mathrm{lacZ} \mathrm{M} 15 \Delta(\text { lacZYA- } \\
\text { argF)U169 deoR recA1 } \\
\left.\text { endA1 hsdR17 (rk mk } \mathrm{mk}^{+}\right) \\
\text {phoA supE44 } \lambda^{-} \text {thi-1 gyrA96 } \\
\text { relA1 }\end{array}$ & $\begin{array}{l}\text { New } \\
\text { England } \\
\text { Biolabs }\end{array}$ \\
\hline \multicolumn{4}{|c|}{ Pseudomonas aeruginosa } \\
\hline PAO1 & & Prototypic wild type & $\begin{array}{l}\text { (Stover et } \\
\text { al., 2000) }\end{array}$ \\
\hline PKM900 & PAO1 & $\Delta m i f S(P A 5512)$ & $\begin{array}{l}\text { PAO } \Delta \text { mifS } \\
\text { (Tatke et al., } \\
\text { 2015) }\end{array}$ \\
\hline PKM901 & PAO1 & $\Delta m i f R(P A 5511)$ & $\begin{array}{l}\text { PAO } \Delta \text { mifR } \\
\text { (Tatke et al., } \\
\text { 2015) }\end{array}$ \\
\hline PKM902 & PAO1 & $\Delta$ mifSR (PA5511-PA5512) & $\begin{array}{l}\text { PAO } \Delta \text { mifSR } \\
\text { (Tatke et al., } \\
\text { 2015) }\end{array}$ \\
\hline \multicolumn{4}{|l|}{ Plasmids } \\
\hline pPSV37 & & $\mathrm{Gm}^{\mathrm{R}}$; colE1 oriT lacl ${ }^{\mathrm{q}} \mathrm{P}_{\text {lacuv5 }}$ & $\begin{array}{l}\text { (Lee et al. } \\
2010)\end{array}$ \\
\hline pGDT004 & pPSV37 & $\begin{array}{l}\mathrm{Gm}^{\mathrm{R}} \text {; The mifR ORF } \\
\text { subcloned from pGDT002 as } \\
\text { an Nhel-Sacl fragment into } \\
\text { pPSV37 }\end{array}$ & $\begin{array}{l}\text { (Tatke et } \\
\text { al., 2015) }\end{array}$ \\
\hline pGDT005 & pPSV37 & $\begin{array}{l}\mathrm{Gm}^{\mathrm{R}} \text {; A } ~ 3.0-\mathrm{kb} \text { Nhel-Sacl } \\
\text { fragment containing mifSR } \\
\text { (PA5511-PA5512) ORFs } \\
\text { amplified from PAO1 } \\
\text { genome using HK_mifSF1 } \\
\text { and GDT_mifRR1 primers } \\
\text { and cloned directly into Nhel- } \\
\text { Sacl-cut in pPSV37 }\end{array}$ & $\begin{array}{l}\text { (Tatke et } \\
\text { al., 2015) }\end{array}$ \\
\hline pGDT006 & pPSV37 & $\begin{array}{l}\mathrm{Gm}^{\mathrm{R}} ; \mathrm{A} \sim 1.3-\mathrm{kb} \text { Nhel-Sacl } \\
\text { fragment containing PA5530 } \\
\text { ORF amplified from PAO1 } \\
\text { genome using } \\
\text { GDT_PA5530F1 and } \\
\text { GDT_PA5530R1 primers }\end{array}$ & $\begin{array}{l}\text { (Tatke et } \\
\text { al., 2015) }\end{array}$ \\
\hline
\end{tabular}


and cloned directly into Nhel-

Sacl-cut in pPSV37

$\mathrm{Gm}^{\mathrm{R}}$; A 1.3-kb Nhel-Sacl

fragment containing pcaT

(PA0229) ORF amplified

pGDT007 pPSV37

from $\mathrm{PAO} 1$ genome using

GDT_pcaTF1 and

This study

GDT_pcaTR1 primers and

cloned directly into Nhel-

Sacl-cut in pPSV37

Table 3.2: Primers used in this study.

\begin{tabular}{ll}
\hline \multicolumn{1}{c}{ Primer Name } & \multicolumn{1}{c}{ Sequence } \\
\hline GDT_pcaTF1 & 5'- \\
& GCTAGCAGAAGGAGATATACCATGACTGCCCCTG \\
TTCAC-3' & \\
GDT_pcaTR1 & 5'-TTTGAGCTCCCGTCAGGGCTCGTCGTT-3' \\
GDT_p37_SeqF & 5'-GACCCGTTTAGAGGCCCCAA-3' \\
GDT_p37_SeqR & 5'-CGTGCTTTACACTTTATGCTTCCGG-3' \\
pcaT_seqF & 5'-TTCGTGATCGGCGCGATC -3' \\
pcaT_seqR & 5'-CAGCCAGAGGGCGATCAC-3' \\
DBS_qRT_clpXF & 5'- TGCGATTACGATGTGGAGA -3' \\
DBS_qRT_clpXR & 5'- CCCTCGATGAGCTTCAGCA -3' \\
GDT_qRT_pcaTF & 5'- TGCGATTACGATGTGGAGA -3' \\
GDT_qRT_pcaTR & 5'- CCCTCGATGAGCTTCAGCA -3' \\
GDT_qRT_PA5530F & 5'-CGCAACGCATCAAGTCGAT-3' \\
GDT_qRT_PA5530R & 5'-AGTCGTACCACTCGACCAGGTT-3' \\
qRT_phzA1F & 5'- GGCACAACGTGCGGATCT -3' \\
qRT_phzA2R & 5'- CGCACTCGACCCAGAAGTG-3' \\
qRT_phzA2F & 5'- GCCCTGCGCGAATACG -3' \\
qRT_phzA2R & 5'- CCATTCCTCTTTTTCCCGTTCT -3' \\
qRT_phzSF & 5'- GCCCTGCGCGAATACG -3' \\
qRT_phzSR & 5'- CCATTCCTCTTTTTCCCGTTCT -3' \\
qRT_phzMF & 5'- GAGCGCATCCATCGACTGAT -3' \\
qRT_phzMR & 5'- GGTATCGCCCTGGAAGATCTC -3' \\
\hline
\end{tabular}




\section{Results and Discussion}

Acquisition of energetically favorable carbon and energy sources from the extracellular milieu of nutrients is of paramount importance to bacterial survival and disease progression. The $C_{5}$-dicarboxylate $\alpha-K G$ is a key intermediate of the energy-generating TCA cycle and plays an important role in regulating the cellular carbon and nitrogen metabolism (Doucette et al., 2011). An important aspect of the nutrient acquisition process is their transport from the environment into the cells via the transporter proteins. The transport of $\alpha-K G$ has been studied in only a handful of bacteria including $P$. aeruginosa (Lundgren et al., 2014, Tatke et al., 2015), E. coli (Seol \& Shatkin, 1991, Cai et al., 2013), R. trpopici (Batista et al., 2009), X. oryzae (Guo et al., 2012) and Staphylococcus aureus (Tynecka et al., 2001). In $P$. aeruginosa, E. coli and $R$. tropici extracellular $\alpha-K G$ transport is arbitrated by TCS proteins MifS/MifR (Tatke et al., 2015), KguS/KguR (Cai et al., 2013) and KgtS/KgtR (Batista et al., 2009), respectively. The present study further explores the role of $P$. aeruginosa mifSR encoding the sensor kinase MifS and response regulator MifR in regulating $\alpha-K G$ transport. Furthermore, using the $\mathrm{PAO} \Delta$ mifS, PAO $\Delta$ mifR and $\mathrm{PAO} \Delta$ mifSR mutants and their derivatives, we additionally investigated the role of MifSR TCS and the $\mathrm{C}_{5}$-dicarboxylate $\alpha-K G$ in regulating $P$. aeruginosa virulence.

\section{$P$. aeruginosa possess multiple $\alpha-K G$ transport proteins}

The E. coli transporter KgtP is the most extensively studied $\alpha-K G$ permease (Seol \& Shatkin, 1991, Seol \& Shatkin, 1992, Seol \& Shatkin, 1993). A homology search in $P$. aeruginosa PAO1 genome revealed the presence of two ORFs 
PA0229 (pcaT) and PA5530 encoding the putative dicarboxylic acid transport proteins that are homologs of $E$. coli $\alpha-K G$ permease KgtP. After here we name $P$. aeruginosa PA5530 as KgtP. In recent studies, KgtP has been characterized and identified as the functional $\alpha-K G$ transporter, whose expression is regulated by the MifSR TCS in coordination with the alternate sigma factor RpoN $\left(\sigma^{54}\right)$ (Lundgren et al., 2014, Tatke et al., 2015). In P. puitda, PcaT has been implicated in the transport of $\beta$-ketoadipate (Ondrako \& Ornston, 1980). However, in P. aeruginosa PcaT function was not previously explored.

Our sequence analyses of $P$. aeruginosa PcaT and KgtP with E. coli KgtP using BLASTP revealed that $P$. aeruginosa PcaT (PA0229) is the closest $E$. coli KgtP homolog with $88 \%$ similarity and $70 \%$ identity, suggesting a similar function. P. aeruginosa PcaT and KgtP share $72 \%$ similarity and $55 \%$ identity, and have the conserved protein domain family PRK10406 implicated in a-KG transport (Marchler-Bauer et al., 2015). This data strongly suggests that $P$. aeruginosa PcaT is capable of transporting $\alpha-K G$ into the cells. To confirm the role of $P$. aeruginosa pсaT in a-KG uptake, pcaT was amplified and subcloned downstream of the inducible $\mathrm{P}_{\text {lacuv5 }}$ promoter to generate the plasmid pPcaT. The plasmid pPcaT was then introduced into the wild-type PAO1 and its isogenic mifSR mutant strains. The $\mathrm{PAO} \Delta$ mifS, PAO $\Delta$ mifR and $\mathrm{PAO} \Delta$ mifSR mutants exhibit an $\alpha-K G-d e p e n d e n t$ growth defect due to the deficiency in the transport of extracellular $\alpha-K G$ (Tatke et al., 2015). Expression of pcaT in trans in the mifSR mutants restored their growth to a level comparable to the wild-type PAO1 in M9 minimal media with a-KG (30 $\mathrm{mM}$ ) as the sole carbon source (Figure 3.1). 


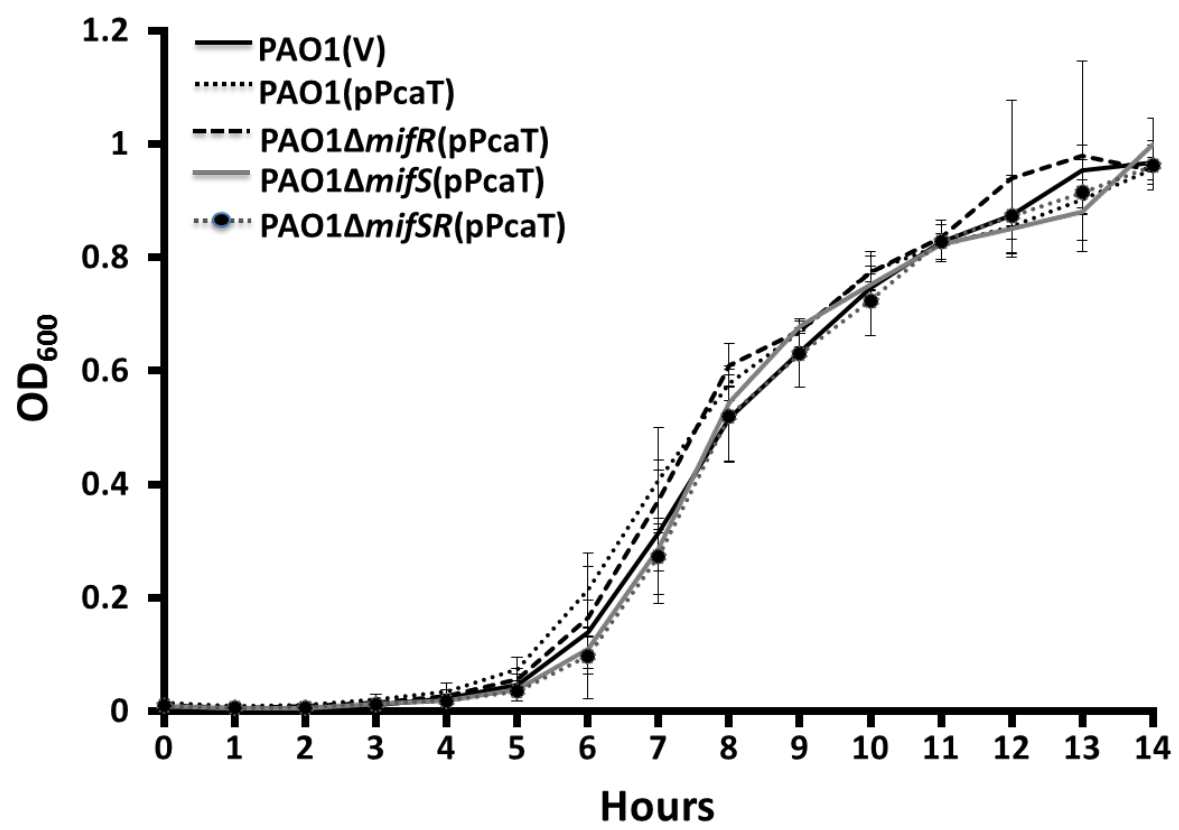

Figure 3.1: Growth of mifSR mutants in the presence of $\alpha-K G$ and pPcaT. Growth curves of $P$. aeruginosa wildtype PAO1 and mifSR mutants expressing pcaT, encoding the putative carboxylic acid transporter in trans in M9 minimal media with a-KG (30 mM). Results shown are mean with standard deviation of three biological replicates.

Table 3.3: Growth Properties of the mifSR mutants and its derivatives in the presence of $\alpha-K G$

\begin{tabular}{ccccc}
\hline & PAO1 & $\Delta$ mifR & $\Delta$ mifS & $\Delta$ mifSR \\
EV & +++ & --- & -- & -- \\
pPcaT & +++ & +++ & +++ & +++ \\
pPA5530 & +++ & +++ & +++ & +++
\end{tabular}


Furthermore, growth of the wild-type PAO1 was not affected by the expression of an extra copy of the pcaT gene (Figure 3.1). This finding confirms the role and ability of $P$. aeruginosa PcaT in transporting extracellular $\alpha-K G$ into the cells.

Biochemical studies have hinted to the presence of inducible $\alpha-K G$ transporters in Pseudomonas spp (Campbell \& Stokes, 1951, Edwards et al., 1979). This was supported by our recent findings where the expression of $P$. aeruginosa kgtP gene encoding the $\alpha-K G$ permease was significantly induced by $\alpha-K G$ (Tatke et al., 2015). To test if the expression of pcaT gene is induced by the presence of extracellular $\alpha-K G$, qRT-PCR analysis was performed. Expression of pcaT was tested in the wild-type PAO1 grown with varying amounts of $\alpha-K G$ (1 $80 \mathrm{mM})$. The presence of $\alpha-K G$ resulted in the activation of $p c a T$ expression in a concentration independent manner (Figure 3.2). This data suggests that pcaT expression is induced by $\alpha-K G$.

The mifSR mutants failure to grow in the presence of $\alpha-K G$ is the result of a defect in the expression of $\alpha-K G$ transporter proteins (Tatke et al., 2015). This growth deficient phenotype can be rescued by uncoupling the MifSR regulatory control over pcaT and $k g t P$ by expressing these genes under a regulatable promoter (Table 3.3). This suggests that MifSR TCS, similar to $\mathrm{kgtP}$, regulates pcaT expression. To determine if this is true, we looked at the pcaT and $k g t P$ RNA levels in the $\Delta$ mifS, $\Delta m i f R$ and $\Delta$ mifSR mutant strains. Corroborating our previous findings (Tatke et al., 2015), expression of $k g t P$ was downregulated in the mifSR deficient strains (Figure 3.3). However, surprisingly, pcaT expression was upregulated in the absence of mifSR and the presence of a-KG (Figure 3.3). This 
suggests that MifSR TCS negatively regulates pcaT expression. In the experimental set up, the regulatory control of pcaT was uncoupled by expressing it under $\mathrm{P}_{\text {lac UV5. }}$ In the mifSR mutants, there is an intact $p c a T$, however it is unable to rescue the a-KG dependent growth defect (Table 3.3), despite the increased expression (Figure 3.3). This implies that perhaps pcaT expression is controlled post-transcriptionally by an un-identified factor which requires intact MifSR. These findings strongly suggests that MifSR TCS inversely regulates the expression of $P$. aeruginosa pcaT and $\mathrm{kgtP}$.

Our sequence analysis of the pcaT upstream promoter $\left(\mathrm{P}_{p c a T}\right)$ region revealed the presence of a putative weak RpoN $\left(\sigma^{54}\right)$ sigma factor binding $-24 /-12$ sequence: TGGCAC-N7-TCGCC (Barrios et al., 1999) and an A-rich motif adjacent to the ribosome binding site with a signature sequence (AAc/uAAc/A) for the catabolite repression control (Crc) protein (Figure 3.4) (Sonnleitner et al., 2009, Browne et al., 2010). Crc is a post- transcriptional inhibitor that prevents translation initiation by binding to the mRNA (Moreno et al., 2007, Sonnleitner et al., 2009). In addition, we identified four putative GGA trinucleotide motifs in the $P_{p c a T}$, a characteristic binding site recognized by the post-transcriptional control RsmA family of proteins (Brencic \& Lory, 2009). Also, in the predicted secondary structure of the pcaT leader sequence using Mfold we found that the GGA motifs are exposed in the steam-loop structures which could acts as a substrate for RsmA binding, as has been observed for several known RsmA targets (Figure 3.4). Though the role of $\mathrm{Crc}$ and RsmA in $\alpha-K G$ transport has to be verified 
experimentally, it suggests an additional layer of regulation controlling pcaT expression.

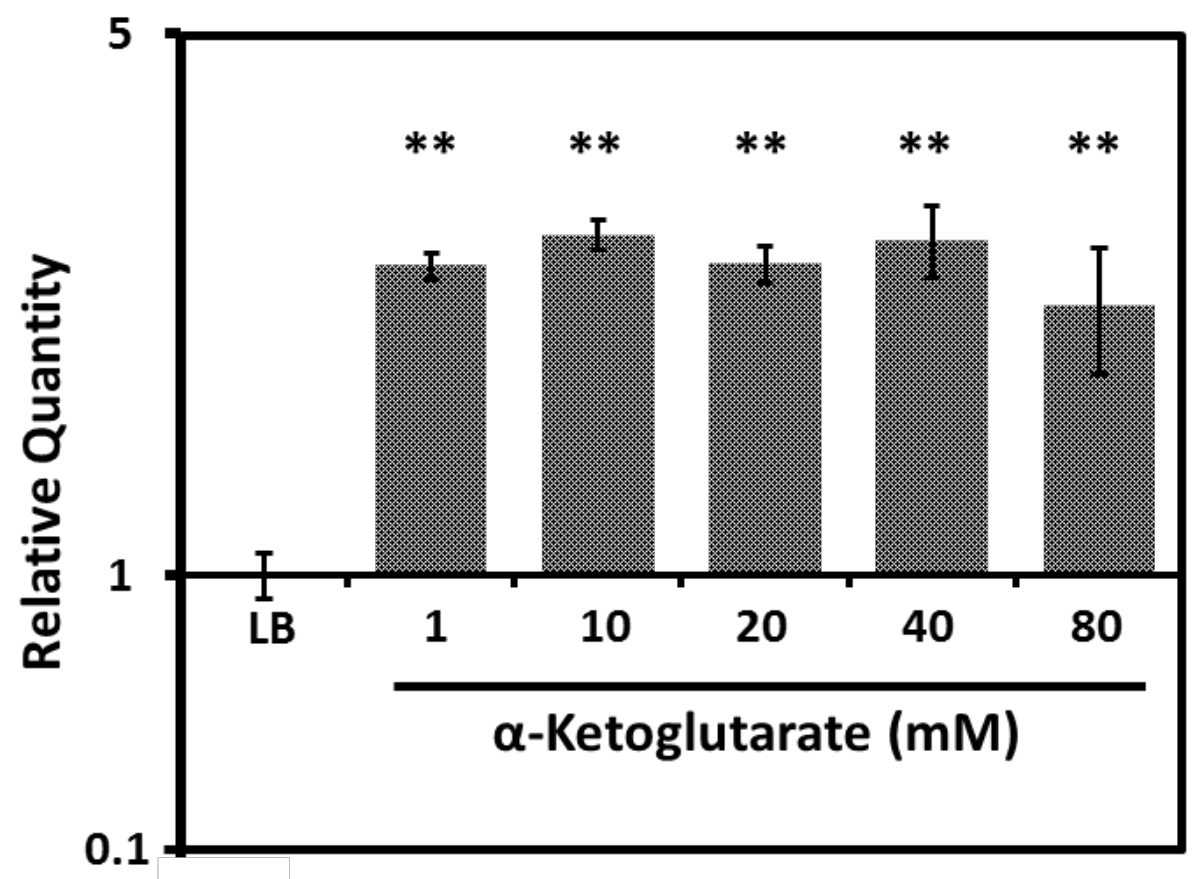

Figure 3.2: pcaT gene expression in response to $\alpha-K G$. qRT-PCR was used to measure the relative quantities of pcaT mRNA after $P$. aeruginosa PAO1 cells were induced for an hour with varying concentration of $\alpha-K G$ (1 to $80 \mathrm{mM}$ ). Bars above or below the line represents up- and down-regulation, respectively, and the error bars indicate standard errors. Data were normalized to expression in PAO1 uninduced cells. The $c / p X$ gene (PA1802) was used as the housekeeping control. Statistically significant difference as determined by oneway ANOVA with Bonferroni's post-hoc test, ${ }^{* *} p$-value $<0.001$ compared with expression in the uninduced condition. 


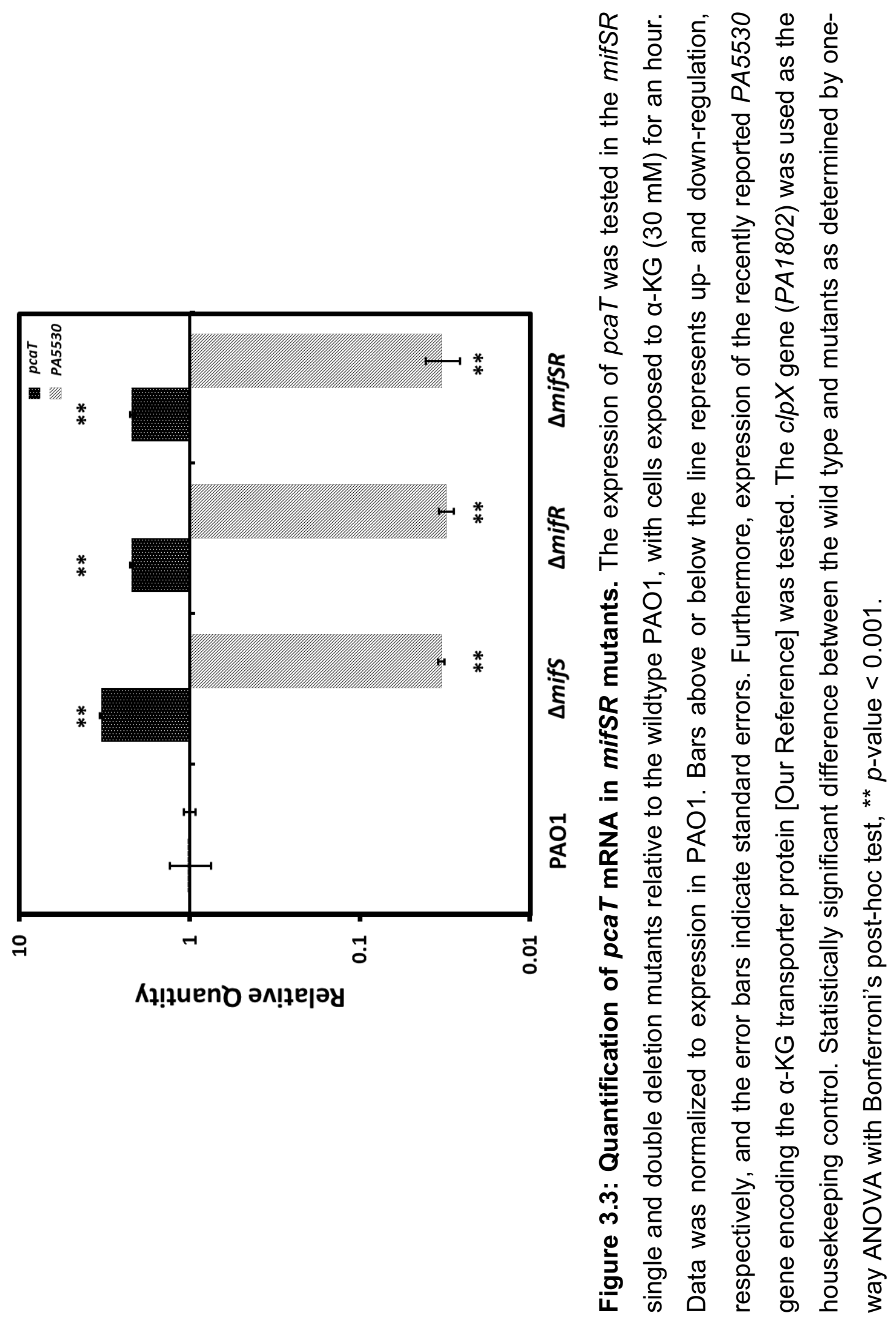



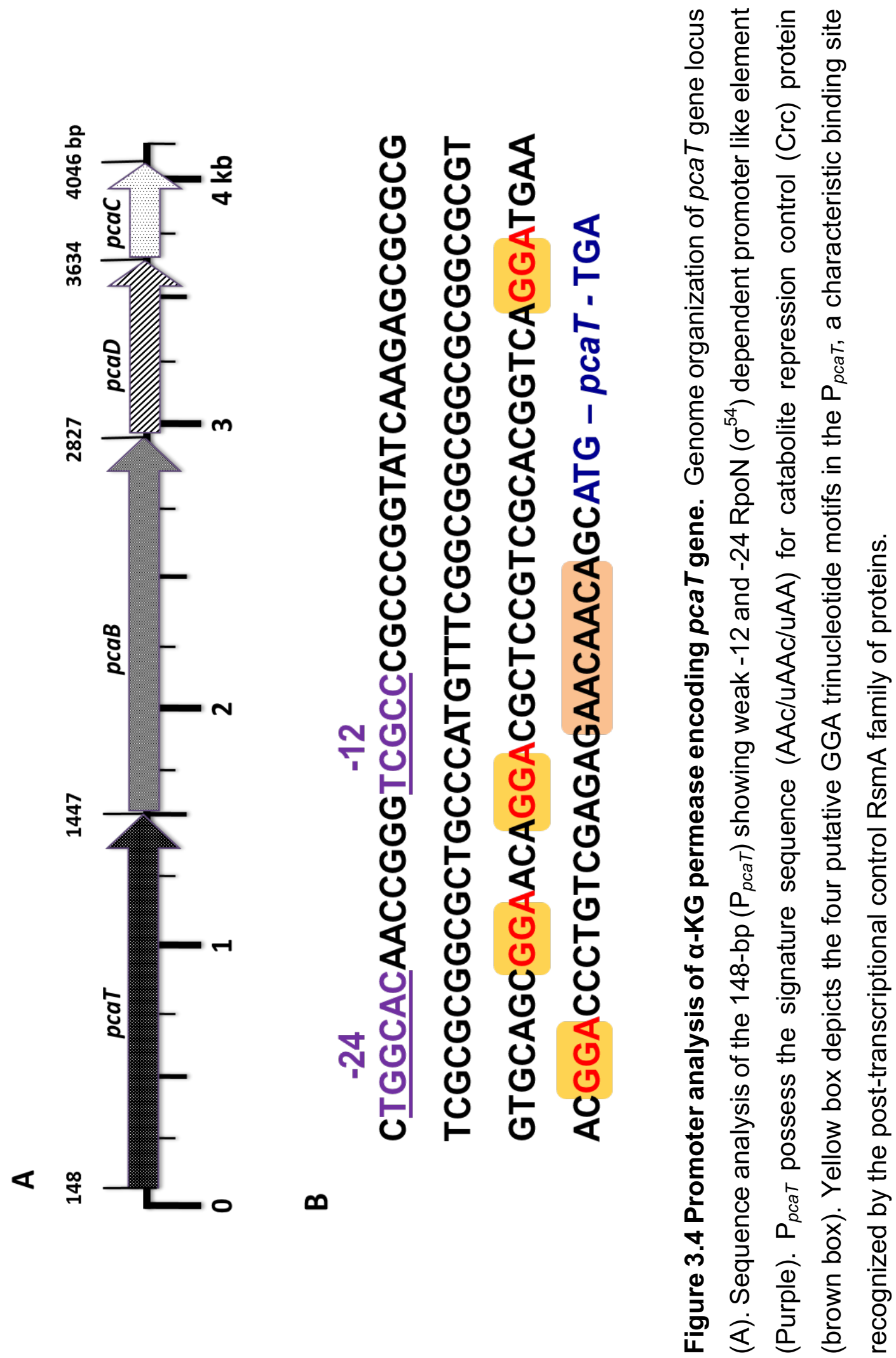


\section{MifS/MifR TCS regulates $P$. aeruginosa virulence}

Success of $P$. aeruginosa as a recalcitrant pathogen relies significantly on its metabolic flexibility, which is exhibited by its ability to utilize a myriad of organic compounds as carbon and energy source for its survival and growth. Furthermore, $P$. aeruginosa is conspicuous for its ability to cause both acute and chronic infections in immunocompromised patients (Bodey et al., 1983). This versatility is manifested in part by expression of a vast array of virulence determinants, both cell-associated and secretory, that are capable of overpowering the host cell defenses and catabolizing macromolecules (Gellatly \& Hancock, 2013). It is therefore not surprising that the synthesis and regulation of multiple virulence factors is controlled by nutrient availability. Carbon and nitrogen sources have been shown to influence several virulence related phenotypes in $P$. aeruginosa including biofilm development (Klausen et al., 2003(a), Shrout et al., 2006), motility (Kohler et al., 2000), pigment production (Palmer et al., 2007, Dandekar et al., 2012) and cell cytotoxicity (Bains et al., 2012). To date, very little is known of the physiology of $P$. aeruginosa when provided with $\alpha-K G$ as the sole carbon source and its role in virulence. Importantly, it is now known that extracellular $\alpha-K G$ is a primary carbon source for some pathogenic bacteria during infection (Guo et al., 2012, Cai et al., 2013). Considering these findings, we further explored if a-KG has a more profound physiological role in regulating $P$. aeruginosa virulence and if MifSR TCS play any role. An impediment in performing experiments with mifSR mutants in the presence of $\alpha-K G$ was there in\#ability to grow in media with $\alpha-K G$ as the sole carbon source (Table 3.3). To circumvent the problem, all the virulence 
related phenotypic assays were performed using $\Delta$ mifS, $\Delta$ mifR and $\Delta$ mifSR mutants harboring pPcaT plasmid, unless specified otherwise. Thus, in the mifSR mutants, in trans expression of the $\mathrm{a}-\mathrm{KG}$ transporter PcaT ensures the influx of extracellular $\alpha-K G$ into the cells while manifesting the effect of mifSR deletions on $P$. aeruginosa virulence phenotypes.

\section{Enhanced biofilm formation in the absence of $m i f S R$}

Biofilm mode of growth is one of the hallmark of $P$. aeruginosa chronic infections (Tolker-Nielsen, 2014). Subsequently, biofilm infections are difficult to eradicate as a result of biofilm's inherited tolerance to clinically significant antibiotics (Costerton et al., 1999). It has been long known that TCS signaling proteins play a crucial role in modulating $P$. aeruginosa biofilm formation (Mikkelsen et al., 2011). The response regulator MifR, has been implicated in regulating $P$. aeruginosa biofilm formation, as mifR-deficient strains failed to form mature biofilms due to a defect in microcolony formation (Petrova \& Sauer, 2009). Furthermore, previous studies have reported the interdependence of pyruvate fermentation and requirement of functional MifR in supporting microcolony formation (Petrova et al., 2012). Considering the above results in the light of previous findings (Tatke et al., 2015) we investigated the impact of MifSR TCS on $P$. aeruginosa biofilm formation in the presence of $\alpha-K G(30 \mathrm{mM})$ as the sole carbon source. As mentioned before, because of the inability of the PAO $\Delta$ mifR, PAO $\triangle$ mifS and PAO $\Delta$ mifSR mutants to grow in the presence of $\alpha-K G$ (Table 3.3) (Tatke et al., 2015), biofilm formation was assessed using mifSR mutant strains harboring the pPcaT plasmid, unless otherwise specified. Also, to rule out the 
possible role of pPcaT affecting biofilm formation, the wild-type strain PAO1 expressing PcaT in trans was used as a control.

Compared to the wild-type PAO1 strain, PAO $\Delta$ mifR(pPcaT), $\mathrm{PAO} \Delta m i f S(\mathrm{pPcaT})$ and $\mathrm{PAO} \Delta m i f S(\mathrm{pPcaT})$ mutants exhibited an increase in biofilm formation in the presence of $\alpha-K G$ as carbon source (Figure 3.5A). The difference was significant at all time points $(P$-value $>0.005)$ tested over a period of 72 hours. Importantly, expression of the wild-type mifR and mifSR genes in trans in the PAO $\Delta$ mifR and $\mathrm{PAO} \Delta$ mifSR mutants restored the biofilm formation to the wild-type PAO1 levels (Figure 3.5A). Furthermore, the presence of pPcaT itself did not have any adverse effect on biofilm formation, as PAO1(pPcaT) formed comparable level of biofilm as that of the wild-type PAO1 without pPcaT (Figure 3.5A). Additionally, our positive control PAOAretS a hyper biofilm forming strain and the negative control PAO $\Delta p e l \Delta p s / \Delta a l g D$ that forms no biofilms showed the expected results (Figure 3.5).

We have previously shown that MifSR TCS is specific for regulating $P$. aeruginosa a-KG transport, as mifS, mifR and mifSR mutants exhibited no growth difference compared to the wild-type PAO1 in the presence of TCA cycle $\mathrm{C}_{4^{-}}$ dicarboxylate (succinate, fumarate, malate) and/or the $\mathrm{C}_{6}$-tricarboxylate (citrate) intermediates (Tatke et al., 2015). It was of interest to investigate if the enhanced biofilm formation phenotype of mifSR(pPcaT) mutants is a-KG specific. To test this, biofilm formation ability of the mifSR(pPcaT) mutants was evaluated in the presence of citrate and/or succinate ( $30 \mathrm{mM}$ each) as the sole carbon source. No difference in biofilm formation between the mifSR(pPcaT) mutant strains and the 
wild-type PAO1 was observed over a period of 72 hours in the presence of succinate (Figure $3.5 \mathrm{~B}$ ) or citrate (Figure $3.5 \mathrm{C}$ ) as a carbon source. These findings suggests that MifSR TCS negatively regulates biofilm formation in an a-KG dependent manner.

It is now well established that the type and availability of nutrients significantly impact biofilm development and composition (Bowden \& Li, 1997, Sauer et al., 2004). In P. aeruginosa, biofilm formation and development progresses distinctly in the presence of different carbon and nitrogen sources (Klausen et al., 2003(a), Klausen et al., 2003(b), Shrout et al., 2006). Most of the studies to date have looked at the influence of citrate, succinate, pyruvate, glutamate, casaminoacids, and sugars on the formation of $P$. aeruginosa biofilms (De Kievit et al., 2001, Klausen et al., 2003(a), Klausen et al., 2003(b), Shrout et al., 2006, Petrova et al., 2012). The present study is one of the first to report $\alpha-K G$ mediated two-component signaling in regulating $P$. aeruginosa biofilm formation. 

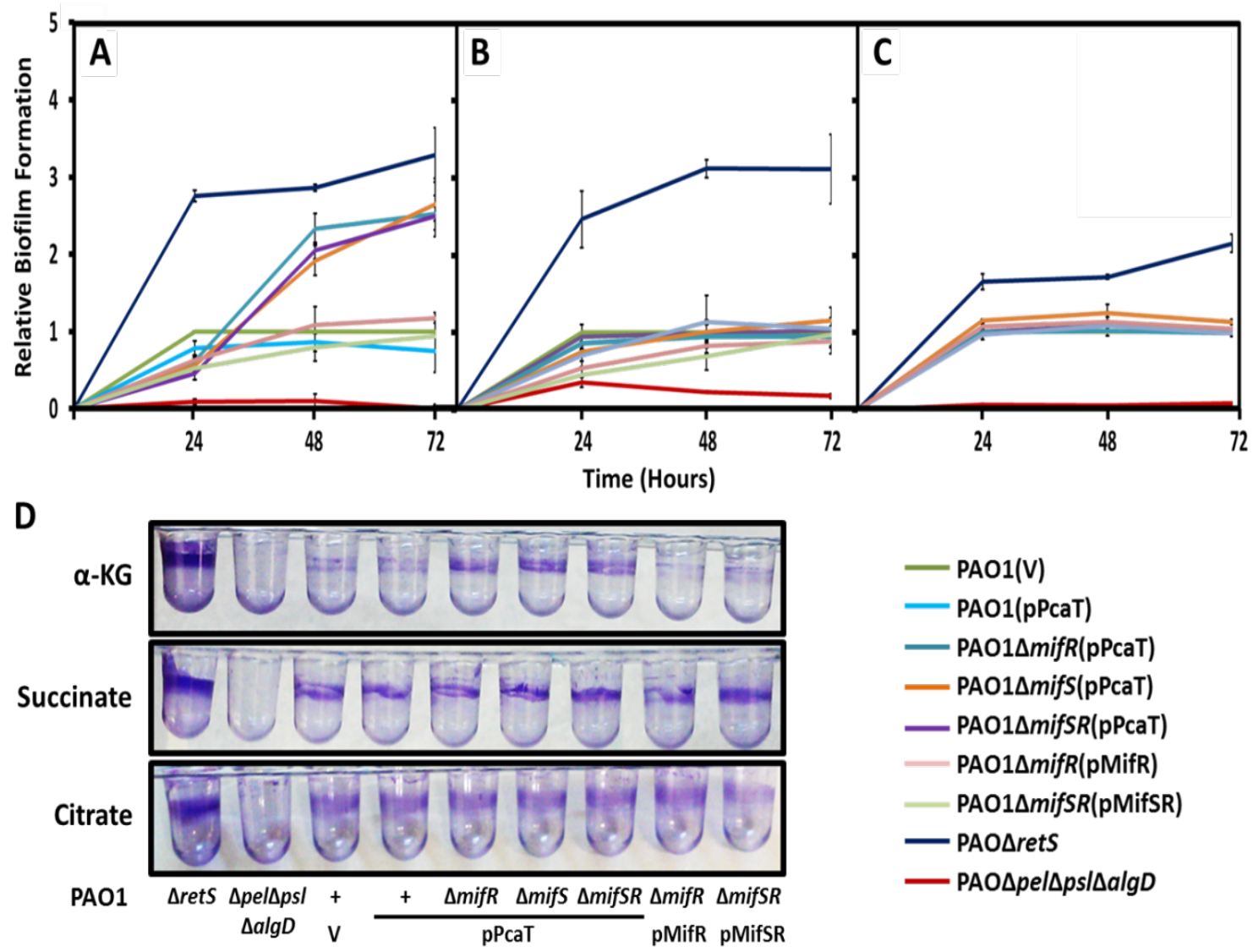

Figure 3.5: $\alpha-K G$ dependent regulation of biofilm formation by mifSR mutants. Biofilm formation of the wild-type PAO1 and mifSR mutants expressing pcaT in trans, hyperbiofilm producing PAOAretS (positive control), and less biofilm producing $\mathrm{PAO} \Delta p e / \Delta p s / \Delta$ alg $D$ (negative control) in M9 minimal media with $\alpha-K G(A)$, succinate $(B)$ and citrate $(C)$ at $30 \mathrm{mM}$ each, as a sole carbon source. The data shown is the relative biofilm formation of each mutant and complementing strain as compared to that of the wild type PAO1 as described in material and methods. Each result represents the mean with standard deviation of three biological replicates. The inset, taken at 72 hours, demonstrates the superior biofilm formation capacity of PAOAmifS(pPcaT), $\mathrm{PAO} \Delta m i f R(\mathrm{pPcaT}), \mathrm{PAO} \Delta$ mifSR (pPcaT) mutants compared to PAO1 in the presence of $\alpha-K G(D)$. Statistically significant difference between the wild type and mutants as determined by one-way ANOVA with Bonferroni's post-hoc test, ${ }^{* *} p$-value $<0.001$. 


\section{mifSR deletion mutants are defective in swarming motility}

In pathogenic bacteria including $P$. aeruginosa, motility facilitates successful invasion, survival and colonization within a host, increases nutrient acquisition efficiency and helps challenging or evading the host defenses, thus playing an indispensable role in its virulence (Kazmierczak et al., 2015). Depending on the growth conditions and medium, $P$. aeruginosa exhibits three types of motility, namely swimming, swarming and twitching (Rashid \& Kornberg, 2000). The motility is controlled by two cell-surface appendages, a single polar flagellum and type-IV pili (Harshey, 2003). The flagellum enables swimming in liquid environments (Toutain et al., 2005) whereas type-IV pili mediates twitching across solid surfaces (Burrows, 2012). Swarming is a coordinated rapid movement over semi-solid or viscous surfaces requiring both flagella and type-IV pili (Kohler et al., 2000). In P. aeruginosa, motility plays a crucial role in facilitating the transition from a free-living planktonic growth to the more sessile biofilm mode of growth (Sauer et al., 2002).

On the basis of our findings that $P$. aeruginosa mifSR-deficient strains form hyper-biofilms in the presence of $\alpha-K G$, we hypothesized that MifSR TCS is involved in regulating motility. To test this, we compared swimming, swarming and twitching on $0.3 \%, 0.6 \%$ and $1 \%$ agar in the wild-type PAO1 and PAO $\Delta$ mifR, $\mathrm{PAO} \Delta$ mifS and $\mathrm{PAO} \Delta$ mifSR mutants harboring the pPcaT plasmid, in the presence of $\alpha-K G(30 \mathrm{mM})$ as the sole carbon source. The mifSR(pPcaT) mutants demonstrated no significant defect in the flagellum-mediated swimming and typeIV pili mediated twitching motilities in the presence of $\alpha-K G$ (Figure 3.6). 


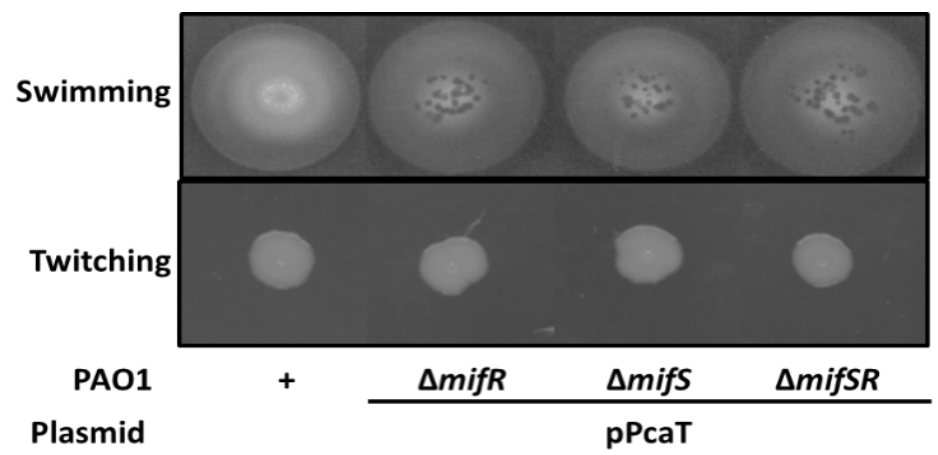

Figure 3.6: Swimming and twitching motility of mifSR mutants in the presence of $\boldsymbol{\alpha}-\mathrm{KG}$. Swimming and twitching motility of the wild-type PAO1, $\mathrm{PAO} \Delta m i f S(\mathrm{pPcaT}), \mathrm{PAO} \Delta m i f R(\mathrm{pPcaT}), \mathrm{PAO} \Delta m i f S R$ (pPcaT) was assessed on media supplemented with $\alpha-K G(30 \mathrm{mM})$. The experiment was repeated multiple times with biological replicates.

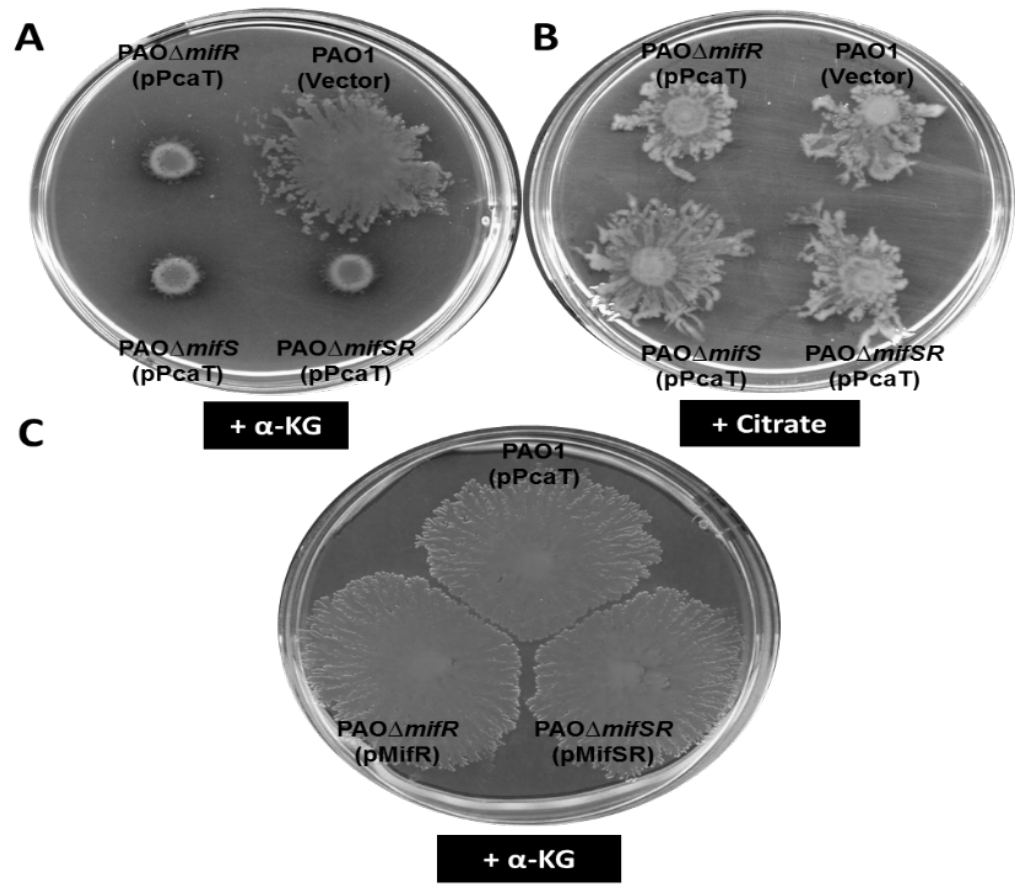

Figure 3.7: $\alpha-K G$ specific swarming motility in mifSR mutants. Swarming motility of the wild-type PAO1, PAO1(pPcaT), PAOAmifS(pPcaT), $\mathrm{PAO} \Delta$ mifR(pPcaT), PAO $\Delta$ mifSR (pPcaT) mutants and the pMifR and pMifSR complementing clones was assessed on media supplemented with $\alpha-K G$ or citrate (30 mM each). The experiment was repeated multiple times with biological replicates. 
However, compared to $P$. aeruginosa wild-type PAO1, the ability of $\triangle m i f S(\mathrm{pPcaT})$, $\Delta m i f R(\mathrm{pPcaT})$ and $\Delta m i f S R(\mathrm{pPcaT})$ mutants to swarm was completely abolished in the presence of $\alpha-K G$ (Figure 3.7A). The swarming defect exhibited by these mutants could be restored to that of the wild-type PAO1 by expressing mifR and mifSR in trans in the $\Delta m i f R$ and $\Delta m i f S R$ mutants (Figure 3.7C). Furthermore, no difference in swarming motility was observed in the PAO1(pPcaT) control strain suggesting that the swarming motility defect in $\Delta m i f S(\mathrm{pPcaT}), \Delta m i f R(\mathrm{pPcaT})$ and $\Delta$ mifSR(pPcaT) mutants is pPcaT-independent and MifSR-dependent.

In our previous study using rich media, such as LB, no role of MifSR TCS was established in regulating $P$. aeruginosa motility (Tatke et al., 2015). The above data further supports the argument that the swarming defect is $\alpha-K G$ dependent. To further investigate, if the inability of mifSR mutants to swarm was a-KG-specific, we tested the ability of these mutants to swarm on other TCA cycle intermediates including succinate and citrate. Interestingly, P. aeruginosa wild-type PAO1 and the mifSR mutants with or without pPcaT exhibited identical swarming phenotypes in the presence of succinate or citrate (Figure 3.7B). Also, no difference in swimming and twitching motility was observed in presence of citrate or succinate as a carbon source. Together, these findings clearly argues for the requirement of a functional MifSR in modulating $P$. aeruginosa swarming motility exclusively in the presence of $\alpha-K G$.

Swarming motility in $P$. aeruginosa is often regulated in response to rhamnolipid production (Kohler et al., 2000, Caiazza et al., 2005). Rhamnolipids are surface-active agents that help in reducing surface tension to drive flagellum- 
mediated translocation over semi-solid surfaces (Kohler et al., 2000). In addition, rhamnolipids have been recovered from CF patient sputa and are involved in inactivation of mammalian tracheal cells and erythrocyte destruction, indicating their importance as virulence factors (Hastie et al., 1986, Kownatzki et al., 1987, Jensen et al., 2007). Lack of swarming in the $\Delta$ mifS(pPcaT), $\Delta m i f R(p P c a T)$ and $\Delta m i f S R(\mathrm{pPcaT})$ mutants can be attributed to a difference in rhamnolipid production in the presence of $\alpha-K G$. To test if the swarming defect in the mifSR mutants is concomitant with rhamnolipid production we performed the rhamnolipid plate assay with $\alpha-K G$ as a carbon source. No significant difference was observed in rhamnolipid production in the $\Delta m i f S(\mathrm{pPcaT}), \Delta m i f R(\mathrm{pPcaT})$ and $\Delta m i f S R(\mathrm{pPcaT})$ mutants as compared to the wild-type PAO1 with or without pPcaT in the presence of $\alpha-K G$ (Figure 3.8). Although interesting, this is not surprising as similar findings have been reported in $P$. aeruginosa where swarming phenotype was independent of rhamnolipid production (Yeung et al., 2011, Guo et al., 2014).

Swarming motility by itself is a complex surface associated behavior controlled by multiple physical factors (flagella, type IV pili and rhamnolipid) (Kohler et al., 2000, Deziel et al., 2003) and transcriptional regulatory networks (Yeung et al., 2009) . Additionally, swarming motility is also dependent on the availability of specific nutrients (Rashid \& Kornberg, 2000, Harshey, 2003, Bains et al., 2012). In $P$. aeruginosa, swarming motility is influenced by the presence of specific carbon and nitrogen sources (Kohler et al., 2000, Bernier et al., 2011). Swarming is augmented in response to nitrogen limitation, and in the presence of certain amino acid such as glutamate, histidine, proline and aspartate (Kohler et al., 2000). 
Interestingly $\alpha-K G$ is central to both these processes, it is a well-established signal for nitrogen starvation (Leigh \& Dodsworth, 2007) and is also a precursor for the synthesis of amino acids glutamate, histidine and proline (Owen et al., 2002). Together, our finding suggests a more intricate network of metabolic control exerted by $\alpha-K G$ on $P$. aeruginosa swarming motility that still needs to be further explored. Furthermore, in $P$. aeruginosa swarming motility and biofilm mode of growth are inversely regulated i.e., strains that are deficient in swarming motility produce more biofilm while strains that exhibit increased swarming motility produce less biofilms (Caiazza et al., 2007). This is achieved by a complex interplay between several regulatory mechanisms including TCSs and the seconder messenger molecule cyclic-di-GMP (Goodman et al., 2004, Caiazza et al., 2007, Kuchma et al., 2007, Merritt et al., 2007, Merritt et al., 2010). It has been shown that the intracellular levels of cyclic-di-GMP influence a wide array of bacterial behaviors, with the common trend being that an increase in the levels of cyclic-diGMP encourages sessile behaviors, such as biofilm formation, while the degradation of cyclic-di-GMP favors motile behaviors, such as swarming. As the mifSR mutant exhibits a swarming defect and a hyperbiofilm phenotype, it will be of interest to examine if MifSR TCS plays a role in regulating the levels of cellular cyclic-di-GMP in the presence of $\alpha-K G$. Regardless, our data is reminiscent of these findings that shows an inverse relationship between biofilm formation and swarming motility modulated through the MifSR signaling cascade. However, at this point, the precise transcriptional regulatory control exerted by MifS/MifR TCS proteins on biofilm formation and swarming motility in the presence of $\alpha-K G$ 
remains to be elucidated. Our study shows that MifSR TCS positively regulates swarming motility and negatively regulates biofilm formation in an $\alpha-K G$ dependent manner.

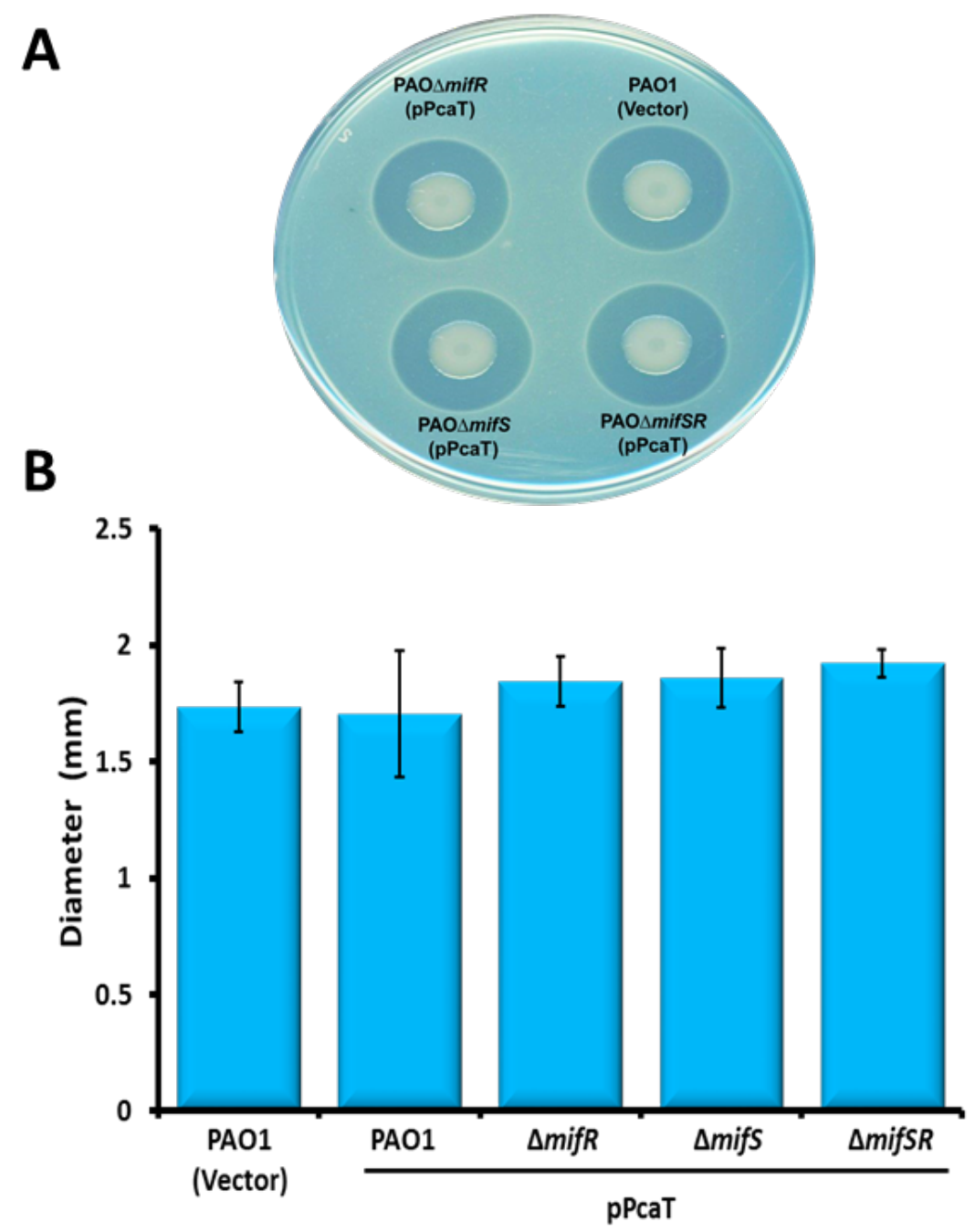

Figure 3.8: Rhamnolipid production in the presence of $\alpha$-KG. Rhamnolipid production of the wild-type PAO1 (Vector), PAO1 (pPcaT), PAOAmifS(pPcaT), $\mathrm{PAO} \Delta$ mifR(pPcaT), PAOAmifSR (pPcaT) was assessed using the cetyltrimethylammonium bromide (CTAB) methylene blue agar plate $(A)$ and the diameter of the blue halo around the colonies was measured as a mark of rhamnolipid production (B) 


\section{Pyocyanin production is negatively regulated by $m i f S R$}

One of the hallmarks of $P$. aeruginosa infections is the production of extracellular, redox-active phenazine compounds. Pyocyanin, a nitrogencontaining heterocyclic blue-green pigment is the major phenazine-derived compound produced by P. aeruginosa (Pierson \& Pierson, 2010). Pyocyanin functions both as a crucial virulence factor (Lau et al., 2004) and as a signaling molecule (Dietrich et al., 2006). Furthermore, pyocyanin exhibits antibiotic properties that allow $P$. aeruginosa to gain dominance over other competing microorganisms, including bacteria and fungi, during infection (Baron \& Rowe, 1981, Kerr et al., 1999, Price-Whelan et al., 2006). Importantly, both in vitro and in vivo studies have revealed that pyocyanin plays an pivotal role in establishing $P$. aeruginosa infections and is essential for its full virulence (Mahajan-Miklos et al., 1999, Ran et al., 2003, Lau et al., 2004, Allen et al., 2005, Cezairliyan et al., 2013). In our earlier experiments when pPcaT was introduced in the $\Delta$ mifS, $\Delta m i f R$ and $\triangle$ mifSR mutants, a visual difference in the pigment production was observed in the presence of $\alpha-K G$ (Figure 3.9). In particular, there was a difference in the production of the blue-green color, a characteristic of pyocyanin (Frank \& Demoss, 1959). This prompted us to ask if pyocyanin production is $\alpha-K G$ dependent and if it is regulated by the MifSR TCS. To test this, we compared the amount of pyocyanin made by $\Delta$ mifS(pPcaT), $\Delta m i f R(\mathrm{pPcaT})$ and $\Delta m i f S R(\mathrm{pPcaT})$ mutants to that of wild-type PAO1 strain in the presence of TCA cycle intermediates including $\alpha-K G$, citrate and succinate ( $30 \mathrm{mM}$ each), as a carbon source. There was no significant difference in the amount of pyocyanin $(\mathrm{ug} / \mathrm{ml})$ produced in the presence 
of citrate and/or succinate between the wild-type PAO1 and the mifSR mutant derivatives (Figure 3.9). However, as compared to the parent PAO1, the mifSR $(\mathrm{pPcaT})$ mutants produced significantly $(p$-value $<0.0005)$ higher amount $(\mathrm{a}$ five-fold increase) of pyocyanin in the presence of $\alpha-K G$ (Figure 3.9). This phenotype was rescued by complementing the mifR and mifSR mutant strains with the wild-type mifR and mifSR genes (Figure 3.9). Also, the comparable amount of pyocyanin produced by the wild-type PAO1 and PAO1(pPcaT) suggests, that the presence of an extra copy of pcaT does not influence this phenotype. Furthermore, absorbance spectra analysis of the overnight cell free culture supernatants showed a characteristic peak of pyocyanin at 310-325 nm (Mavrodi et al., 2001, Reszka et al., 2004). The absorbance peak intensity of the $\Delta m i f S(\mathrm{pPcaT}), \Delta m i f R(\mathrm{pPcaT})$ and $\Delta m i f S R(\mathrm{pPcaT})$ mutants was significantly higher as compared to the wild-type PAO1 strain indicative of more pyocyanin production in the mifSR mutant derivatives (Figure 3.12).These findings strongly suggests an $\alpha-K G$ dependent regulation of $P$. aeruginosa pyocyanin production by the MifSR TCS proteins.

Pyocyanin production in $P$. aeruginosa involves a complex pathway consisting of two homologous operons phzA1B1C1D1E1F1G1 (phzA1) and phzA2B2C2D2E2F2G2 (phzA2) and two additional genes phzM and phzS encoding phenazine modifying enzymes (Mavrodi et al., 2001). To determine if the difference in pyocyanin production in the presence of $\alpha-K G$ is a function of change in gene expression, qRT-PCR was performed to assess the transcript levels of phzA1, phzA2, phzM and phzS genes in the PAO1(pPcaT) and the mifSR(pPcaT) mutants. For the qRT-PCR analysis PAO1(pPcaT) was used as the reference 
strain since it exhibited the same pyocyanin phenotype as that of the wild-type PAO1 strain without pPcaT. In the PAO1 (pPcaT) strain, presence of $\alpha-K G$ appears to inhibit the expression of the phz genes namely, phzA1, phzA2, phzM and phzS (Figure 3.10). The above data suggests that $\alpha-K G$ is a repressor of pyocyanin synthesis in $P$. aeruginosa. In the mifSR(pPcaT) mutant derivatives the expression of phzA1 was significantly induced in the presence of $\alpha-K G$ as compared to the wild-type PAO1(pPcaT). However, the second phzA2 operon and, phzS and phzM genes did not show a significant increase in expression. Pyocyanin production is a characteristic of $P$. aeruginosa stationary growth phase. Here the cells were induced with $\alpha-K G$ for an hour, we suspect that prolong induction of the cells with a-KG might result in a differential expression of the other genes involved in pyocyanin synthesis in the mifSR(pPcaT) mutants. In $P$. aeruginosa PAO1, the expression of phzA1 accounts for majority of phenazine production (Liang et al., 2008), and our qRT-PCR findings suggests that the hyper-production of pyocyanin could be accounted for by an increased expression of phzA1 in the mifSR(pPcaT) mutant strains. Together these findings indicate that MifSR TCS is negatively regulating $P$. aeruginosa pyocyanin production in the presence of $\alpha-K G$. 


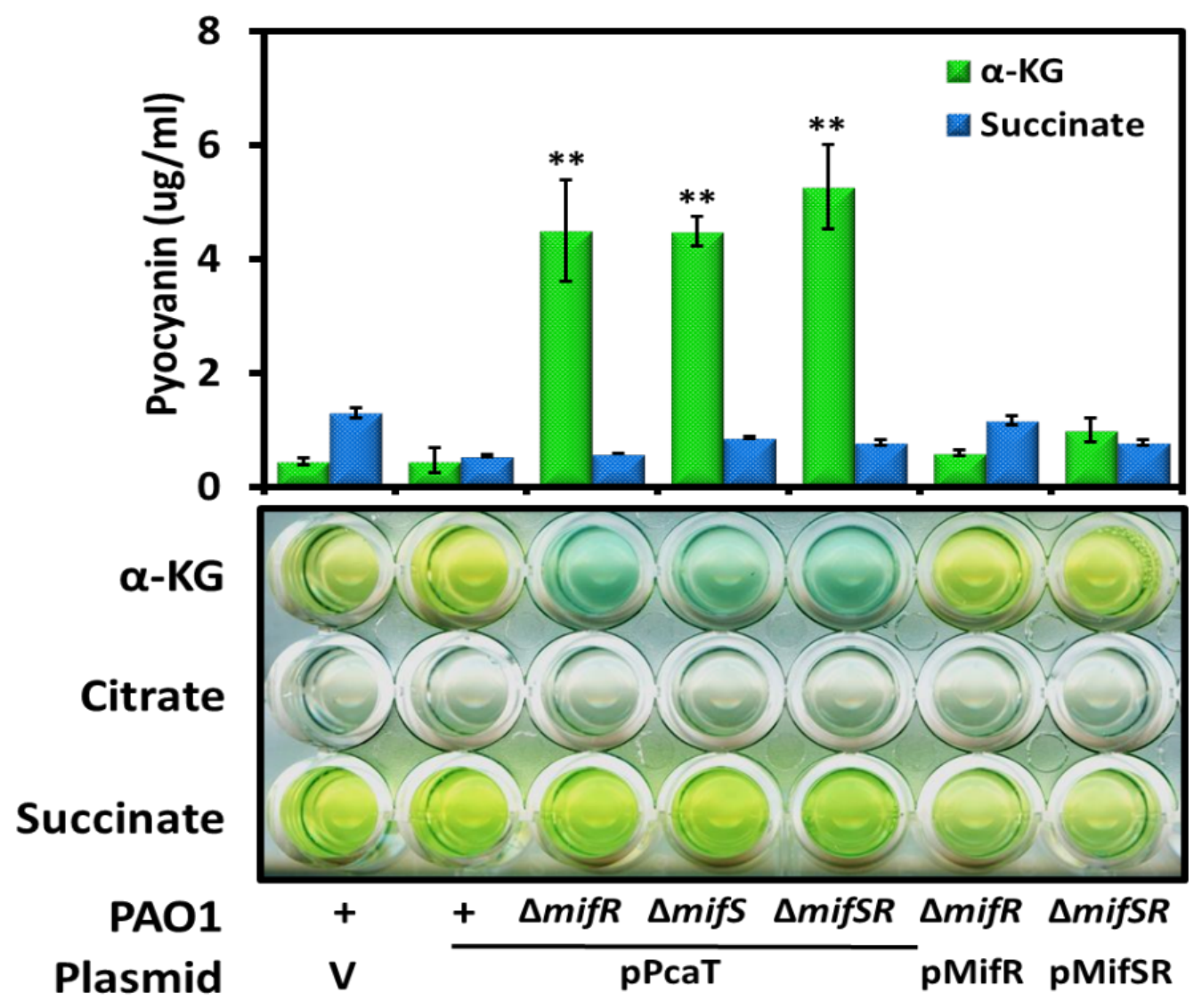

Figure 3.9: Pyocyanin production in mifSR mutants in the presence of a-KG. Pyocyanin production was measured quantitatively from the overnight culture supernatants of the wild-type PA01, PAO1 (pPcaT), PAOAmifS(pPcaT), $\mathrm{PAO} \Delta$ mifR(pPcaT), PAO $\Delta$ mifSR(pPcaT) mutants and the pMifR and pMifSR complementing clones in $\mathrm{M} 9$ minimal media supplemented with $\alpha-K G$, and/or succinate (30 mM each), using the chloroform and HCL method, as described in materials and methods. Results shown are mean with standard deviation of three biological replicates. Statistically significant difference between the wild type and mutants as determined by one-way ANOVA with Bonferroni's posthoc test, ${ }^{* *} p$-value $<0.001$. 


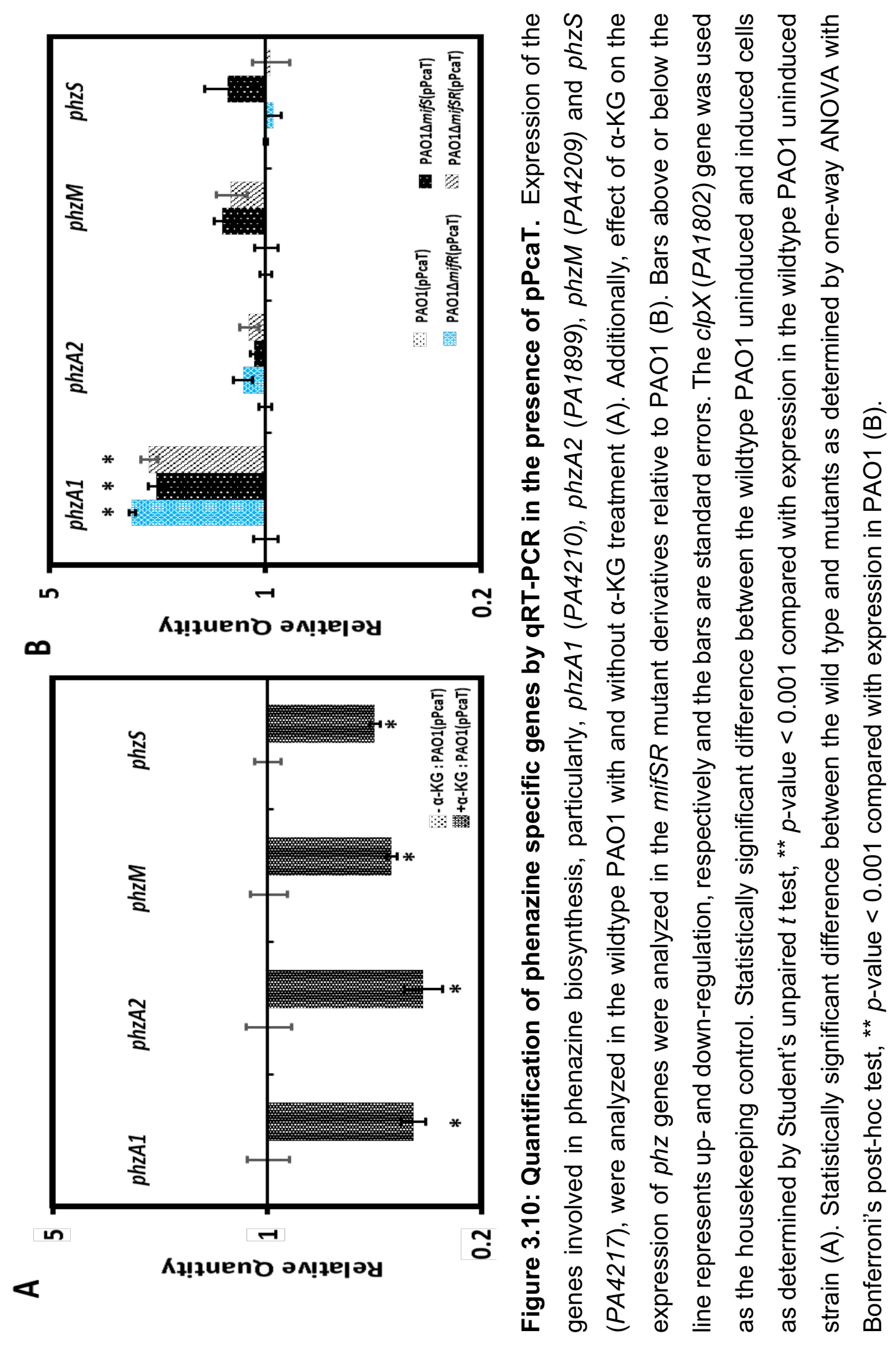




\section{mifSR positively regulates pyoverdine production}

$P$. aeruginosa produces several siderophores that play a pivotal role in scavenging iron from the host, a vital process required for bacterial growth and infection (Bullen, 1981, Poole \& McKay, 2003, Skaar, 2010). Amongst these, pyoverdine, a yellow-green fluorescent pigment is the primary siderophore produced by $P$. aeruginosa (Meyer, 2000, Lamont et al., 2009). Pyoverdine is not only an efficient iron scavenger and transporter during $P$. aeruginosa infection and biofilm formation (Banin et al., 2005, Visca et al., 2007) but also, a signaling molecule established in regulating multiple virulence-associated traits (Beare et al., 2003, Visca et al., 2007). Indeed, several in vivo studies in animal and nematode models demonstrated the requirement of pyoverdine as an important virulence factor essential for successful $P$. aeruginosa infections (Meyer et al., 1996, Takase et al., 2000, Kirienko et al., 2013). Considering the fact that $P$. aeruginosa MifSR TCS is involved in modulating $P$. aeruginosa virulence associated pigment production in the presence of $\alpha-K G$, we investigated its role in pyoverdine production. To test this, we compared the amount of pyoverdine made by $\Delta$ mifS(pPcaT), $\Delta$ mifR(pPcaT) and $\Delta$ mifSR(pPcaT) mutants to that of wild-type PAO1 strain in the presence of TCA cycle intermediates including $\alpha-K G$ and succinate (30 mM each), as a carbon source. As compared to the parent PAO1, the mifSR(pPcaT) mutants produced significantly $(p$-value $<0.005)$ less pyoverdine in the presence of $\alpha-K G$ (Figure 3.11). This phenotype was rescued by complementing the mifR and mifSR mutant strains with the wild-type mifR and mifSR genes (Figure 3.11). No difference in pyoverdine production was observed 
when succinate was provided as the sole carbon source (Figure 3.11) suggesting an $\alpha-K G$ dependent regulation. Also, the comparable amount of pyoverdine produced by the wild-type PAO1 and PAO1(pPcaT) suggests, that the presence of an extra copy of $p c a T$ does not influence this phenotype. These findings strongly suggests an $\alpha-K G$ dependent regulation of $P$. aeruginosa pyoverdine production by the MifSR TCS proteins.

Furthermore, absorbance spectra analysis of the overnight cell free culture supernatants showed a characteristic peak of pyoverdine at 400-405 nm (Vandenende et al., 2004). The absorbance peak intensity of the $\Delta$ mifS(pPcaT), $\Delta m i f R(\mathrm{pPcaT})$ and $\Delta m i f S R(\mathrm{pPcaT})$ mutants was lower as compared to the wildtype PAO1 strain indicative of less pyoverdine production in the mifSR mutant derivatives (Figure 3.12). Together the data suggest that MifSR TCS positively regulates pyoverdine production in the presence of $\alpha-K G$, as the sole carbon source. 


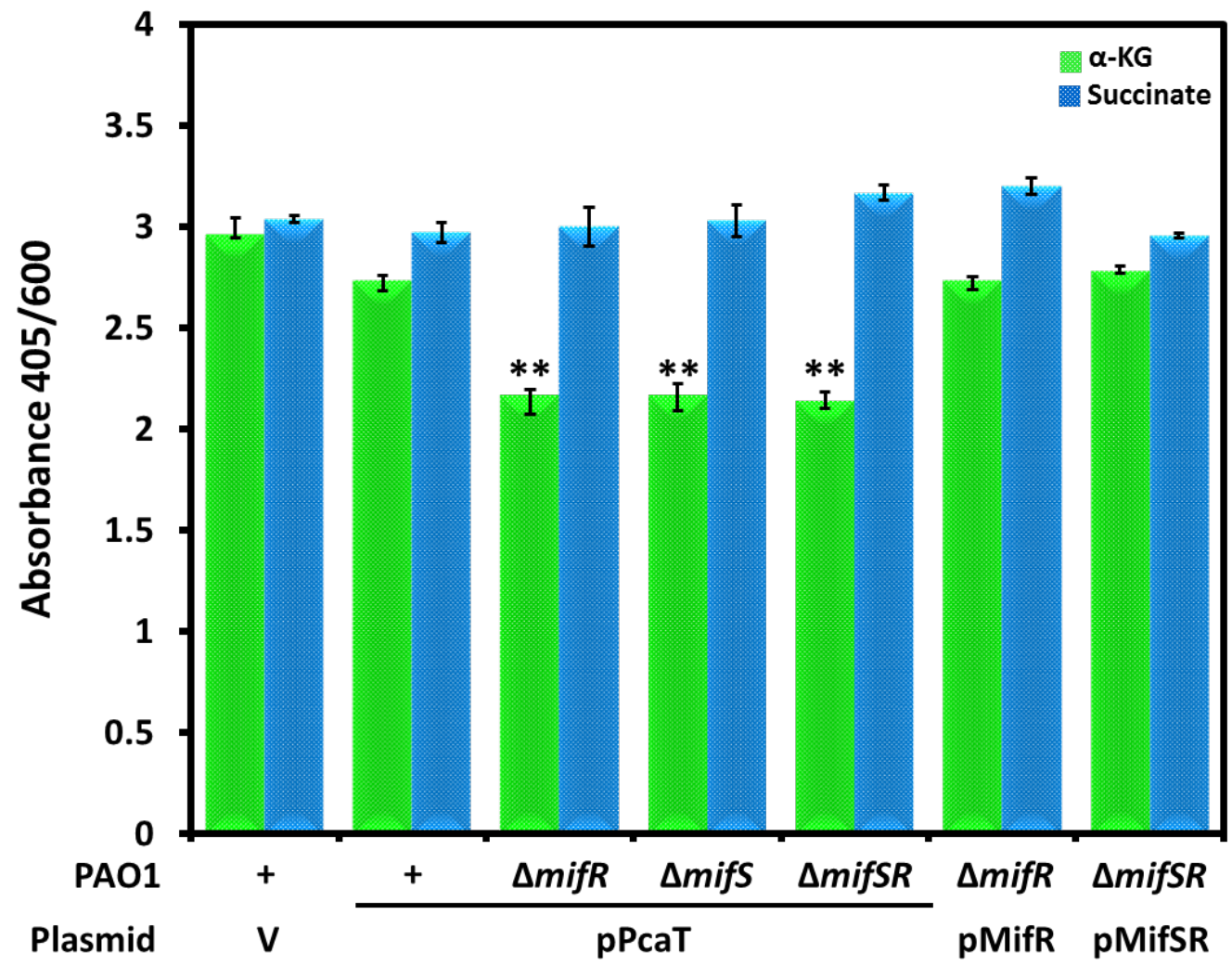

Figure 3.11: Pyoverdin production in mifSR mutants in the presence of $\boldsymbol{\alpha}-K G$. Pyoverdin levels of wild-type PAO1, PAO1(pPcaT), PAOAmifS(pPcaT), $\mathrm{PAO} \Delta m i f R(\mathrm{pPcaT}), \mathrm{PAO} \Delta m i f S R$ (pPcaT) mutants and the pMifR and pMifSR complementing clones in M9 minimal media supplemented with $\alpha-K G$ and/or succinate (30 mM each) was quantitated by measuring the absorbance $A_{405}$. Values are normalized with respect to culture density and $A_{405} / A_{600}$ ratio expressed the pyoverdin levels. Each result represents the mean with standard deviation of three biological replicates. Statistically significant difference between the wild type and mutants as determined by one-way ANOVA with Bonferroni's post-hoc test, ${ }^{* *} p$-value $<0.001$. 


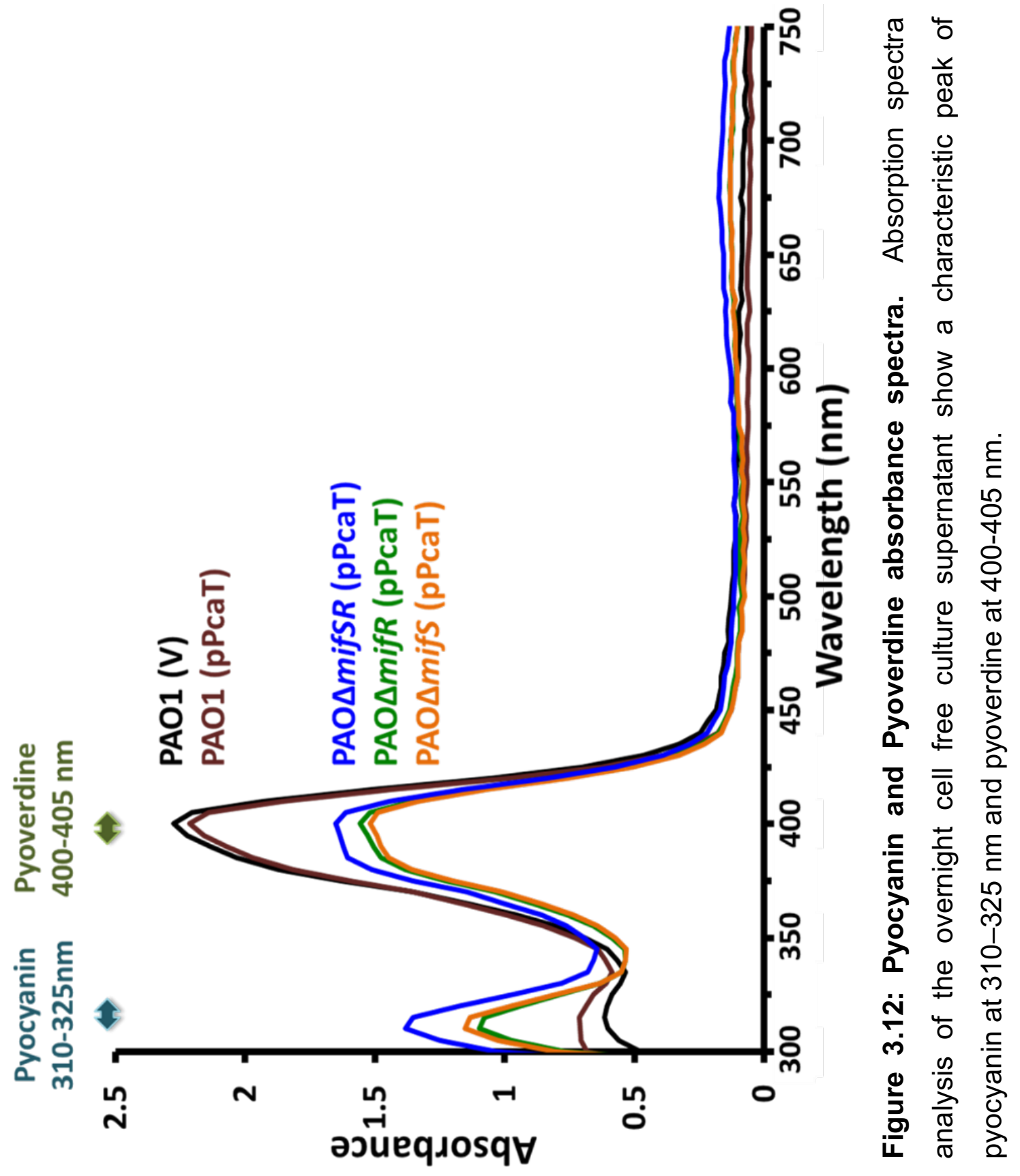




\section{Loss of mifR increases cell cytotoxicity}

$P$. aeruginosa expresses and utilizes an arsenal of virulence mechanisms to gain competitive advantage over its host in-order to establish acute and chronic infections. Most of these mechanisms rely on numerous virulence factors and/or exoproteins, which are intentionally released by this pathogen via diverse and complex secretion machinery. Most of the secreted virulence factors are toxins and hydrolytic enzymes that induce cell cytotoxicity thus causing damage and annihilation of the host cells. Moreover, in a previous microarray gene expression study significant alteration in expression of genes encoding secreted virulence factors was observed in mifR mutant compared to its wild-type PAO1 (Petrova et al., 2012). Since our data indicated that MifSR TCS proteins regulated the transport of extracellular $\alpha-K G$, and modulate different virulence determinants, we examined the ability of mifSR mutant derivatives to infect and destroy A549 lung fibroblast cell cultures in the presence of $\alpha-K G$.

Since all the virulence related phenotypes exhibited by the $\Delta$ mifS(pPcaT), $\Delta m i f R(\mathrm{pPcaT}), \Delta \operatorname{mifSR}(\mathrm{pPcaT})$ mutants were $\mathrm{a}-\mathrm{KG}$ dependent, bacterial cells were grown overnight and sub-cultured in $\mathrm{M} 9$ minimal media with $\alpha-\mathrm{KG}(30 \mathrm{mM})$ before infecting the respective cell cultures. Release of lactate dehydrogenase $(\mathrm{LDH})$ was measured as a function of cell damage using colorimetric LDH activity assay (Hauser \& Engel, 1999). The $\Delta m i f R(\mathrm{pPcaT})$ and $\Delta m i f S R(\mathrm{pPcaT})$ mutants exhibited two-fold increase $(P$-value $<0.005)$ in cytotoxicity compared to the wildtype PAO1 in the presence or absence pcaT (Figure 3.13A). Interestingly no difference in cytotoxicity was observed in case of $\Delta m i f S(\mathrm{pPcaT})$ compared to the 
parent strain PAO1. This could be the result of a potential crosstalk between MifR and other noncognate HKs that needs to be verified. Furthermore, introduction of the wild type mifR and mifSR genes into $\Delta$ mifR and $\triangle$ mifSR mutants restored cytotoxicity to the wild-type PAO1 levels. In-order to rule out the cytotoxicity seen is not specific to A549 lung fibroblast, HeLA and J774-Eclone cells were assessed. All three cell-lines exhibited the same phenotype (Figure 3.14 ) suggesting increased virulence of mifR and mifSR mutants leading to higher cytotoxicity. Subsequently, when $\alpha-K G$ was replaced with succinate as the carbon source, no discernable difference in cell cytotoxicity was observed between the mifSR(pPcaT) mutants and the parent PAO1 strains (Figure 3.13B). The data suggests that MifR independent of MifS, negatively regulates $P$. aeruginosa cell cytotoxicity.

Bacterial pathogens are one of the most portentous infiltrators of the host system, causing significant damage leading to cellular aberrations and injury. In the mammalian cells TCA cycle intermediates including $\alpha-K G$ are mainly found in cytoplasm and mitochondria. Cell injury, results in the efflux of intracellular metabolites leading to an increase in their extracellular concentrations. It is known that TCA cycle intermediates ( $\mathrm{C} 4, \mathrm{C} 5$, and $\mathrm{C} 6$ dicarboxylates) are present at micromolar $(\mu \mathrm{M})$ concentrations in blood that increase with tissues damaged $(\mathrm{He}$ et al., 2004, Hebert, 2004).Therefore it is possible that $\alpha-K G$ might act as a marker of the intensity and level of cellular damage that signals $P$. aeruginosa to downregulate its cytotoxic determinants via mifR regulatory circuit. This is one of the first report of a-KG mediated two-component signaling in regulating $P$. aeruginosa cell cytotoxicity. 


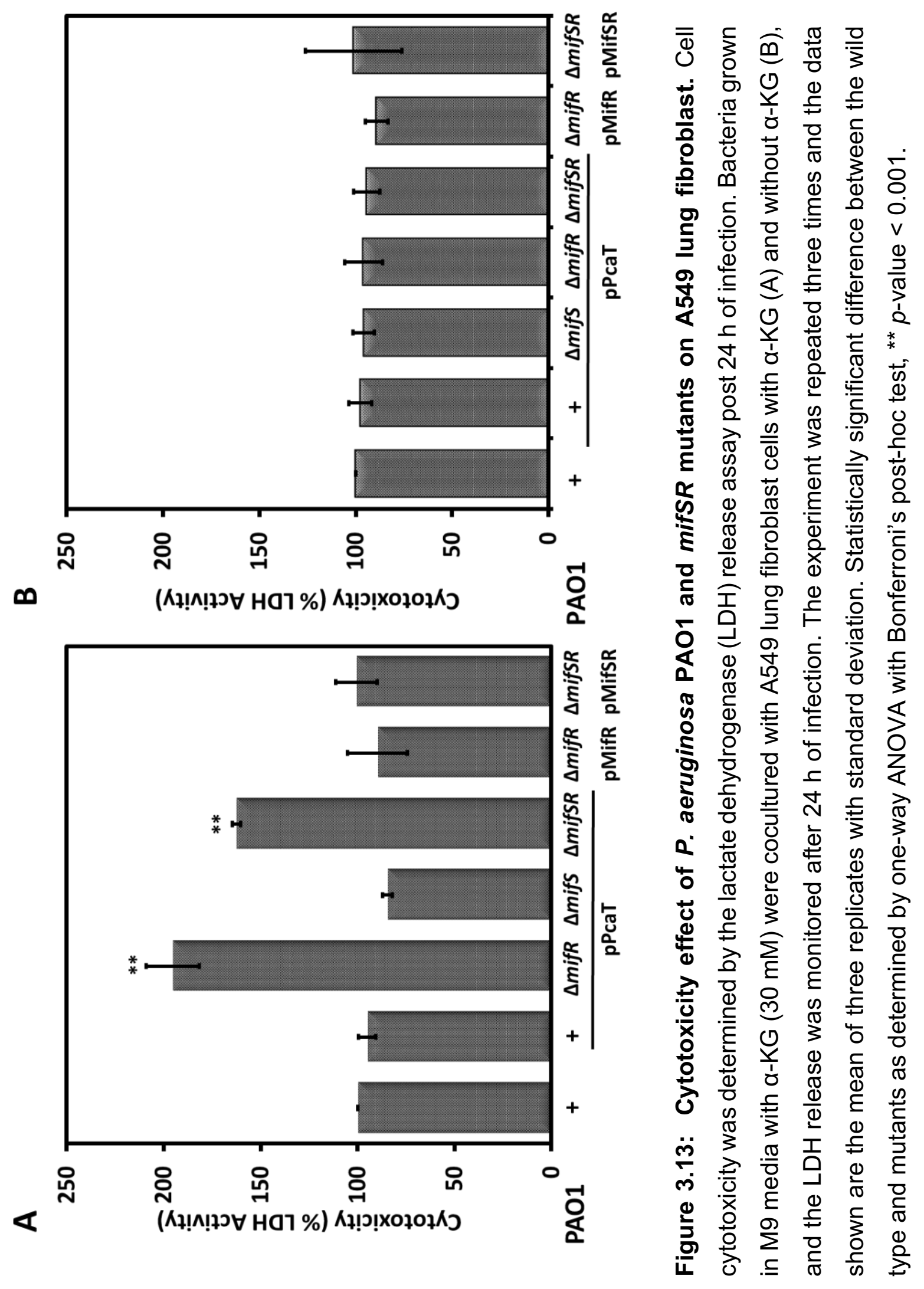




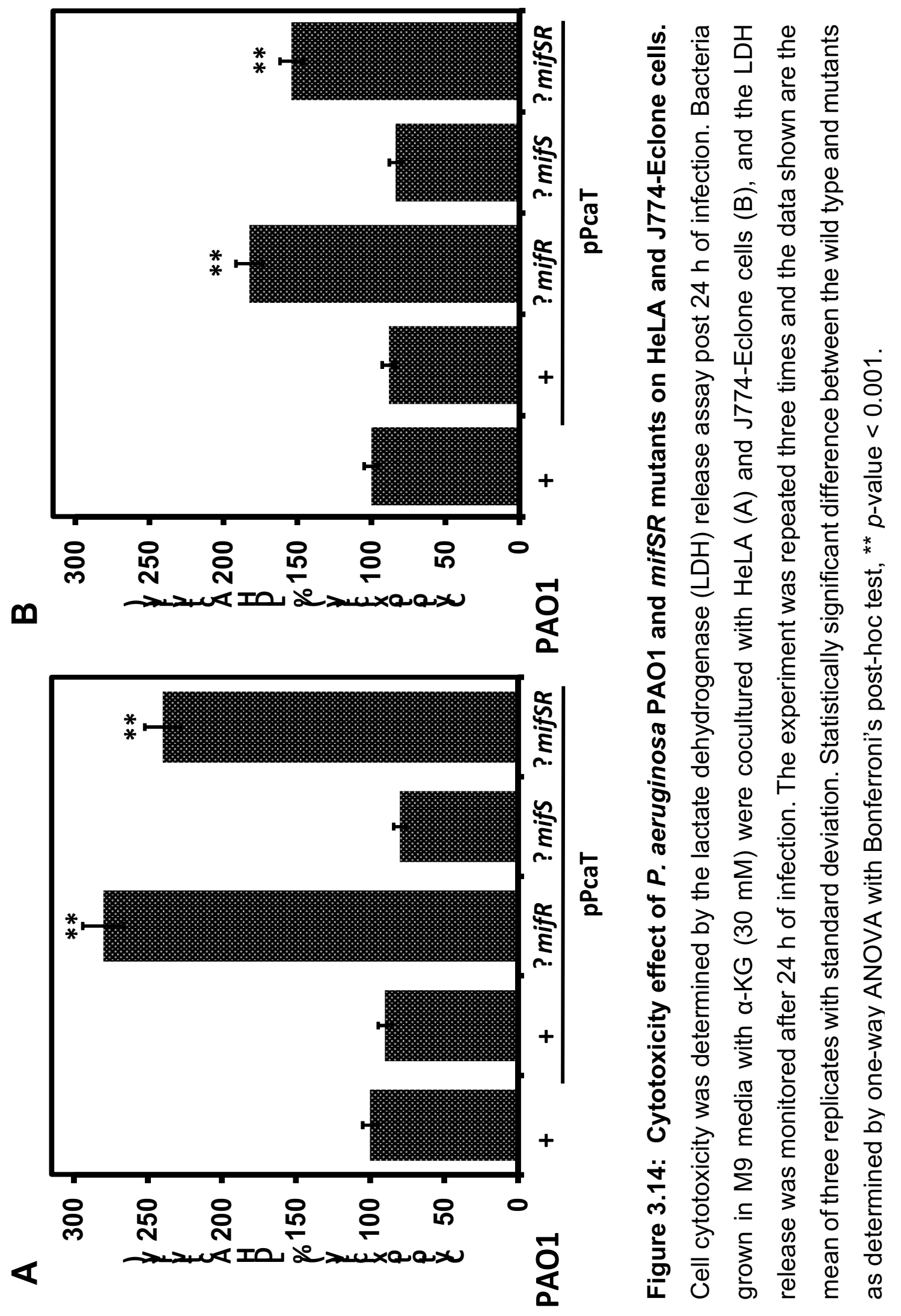




\section{MifSR TCS and $\alpha-K G$ dependent regulation of $P$. aeruginosa virulence.}

To confirm that the expression of $\alpha-K G$ transporter PcaT itself in the mifSR mutants had no effect on $P$. aeruginosa $\alpha-K G-d e p e n d e n t$ virulence phenotypes, we performed pigment (pyocyanin and pyoverdine) production, absorbance spectra analysis and cell cytotoxicity assays with the wild type PAO1 and mifSR mutants in the presence of PA5530 (KgtP) (the previously reported $\alpha-K G$ transporter) (Tatke et al., 2015). Similar to the mifSR(pPcaT) mutants, mifSR(pKgtP) mutants produced significantly $(p$-value $<0.0005)$ higher and lower amounts of pyocyanin and pyoverdine in the presence of $\alpha-K G$, as compared to the parent PAO1 strain with and/or without pKgtP (Figure 3.15A and 3.15B). Furthermore, absorbance spectra analysis of the cell free culture supernatants showed the characteristic peak of pyocyanin at $310-325 \mathrm{~nm}$ and pyoverdine at 400-405 nm. The absorbance peak intensity of the $\Delta$ mifS(pKgtP), $\Delta$ mifR(pKgtP) and $\Delta$ mifSR(pKgtP) mutants was higher for pyocyanin and lower for pyoverdine as compared to the wild-type PAO1 strain (Figure 3.15C). In addition, in the presence of a-KG, similar to the mifSR(pPcaT) mutants, $\Delta m i f R(\mathrm{pKgtP})$ and $\Delta m i f S R(\mathrm{pKgtP})$ exhibited increased cell cytotoxicity, and no difference in cell-cytotoxicity was observed in the case of mifS $(\mathrm{pKgtP})$ compared to the parent PAO1 strain (Figure 3.15D). The mifSR(pKgtP) results corroborates with our mifSR(pPcaT) data, suggesting that mifSR mutant exhibited phenotypes are transporter independent and $\alpha-K G-d e p e n d e n t$. 

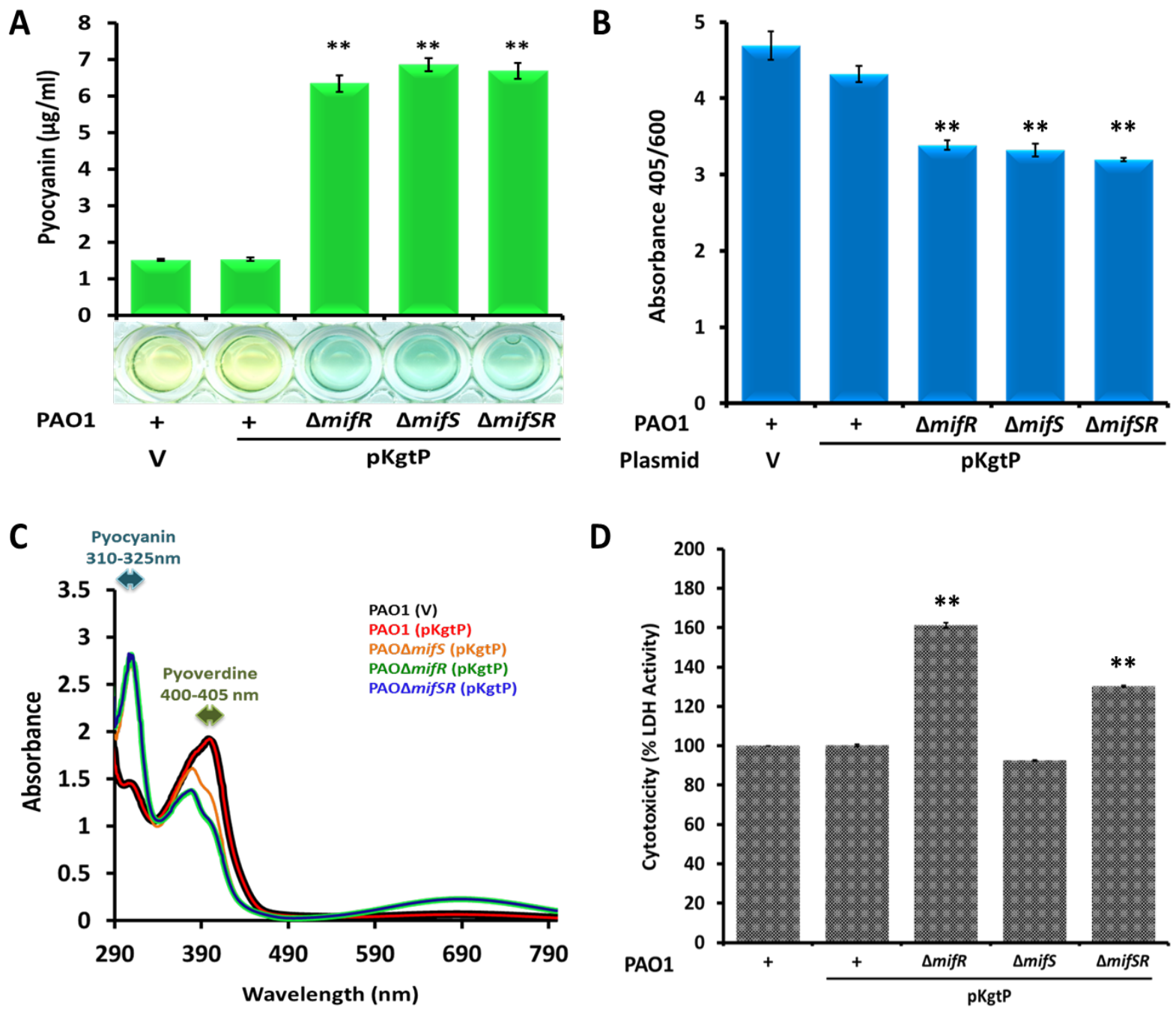

Figure 3.15: $\operatorname{mifSR}(\mathrm{pKgtP})$ virulence assays in the presence of $\alpha-K G$. Pyocyanin production (A), pyoverdine production (B), absorbance spectra (C) and cell cytotoxicity (D) of PAO1, PAO1(pKgtP), mifS(pKgtP), mifR(pKgtP), mifSR(pKgtP) mutants in the presence of $\alpha-K G(30 \mathrm{mM})$. 


\section{Conclusion}

$P$. aeruginosa is one of the most formidable, opportunistic human pathogen infamous for its ability to cause several localized and systemic infections (Tummler et al., 2014). Its ability to colonize different host niches is largely dependent on its metabolic flexibility and environmental adaptability dictated by genetic versatility (Mathee et al., 2008). P. aeruginosa preferentially utilizes TCA cycle intermediates as carbon and energy source over other compounds (Collier et al., 1996), and $\alpha-$ KG is a key intermediate of the TCA cycle (Krebs, 1940). $\alpha-K G$ plays a crucial role in regulating carbon-nitrogen metabolism (Doucette et al., 2011) and importantly, $\alpha-K G$ is considered to be a signaling molecule in both prokaryotes (Ninfa \& Jiang, 2005) and eukaryotes (Lancien et al., 2000, He et al., 2004). Furthermore, recent studies have reported preferential utilization of extracellular a-KG as a principle carbon source during infection by pathogenic bacteria (Guo et al., 2012, Cai et al., 2013). The data together suggests a profound role of $\alpha-K G$ in regulating bacterial physiology and metabolism. However, limited analysis of the physiological roles of a-KG in bacteria, including $P$. aeruginosa, has left its role in pathogenesis unclear. The present study provides a novel insight into the role of $\alpha-K G$ and MifSR TCS in regulating $P$. aeruginosa pathogenesis.

Our findings, suggests a complex regulatory cascade in modulating $P$. aeruginosa $\mathrm{C}_{5}$-dicarboxylate, $\alpha-\mathrm{KG}$ uptake involving a combined action of multiple a-KG transporters namely PcaT (PA0229) and KgtP (PA5530), the MifS/MifR TCS and the sigma factor RpoN (Figure 3.16). It appears that MifS senses the presence of $\alpha-K G$ and signals MifR. The activated-MifR along with the sigma factor RpoN 
initiate the transcription of $\alpha-K G$ transporter genes pcaT and kgtP. Interestingly, our findings suggests that the MifSR TCS inversely regulates the expression of pcaT and KgtP genes (Figure 3). Furthermore, sequence analysis of pcaT promoter $\left(\mathrm{P}_{\text {pсаT }}\right)$ and our previous published $\mathrm{P}_{\text {kgtP }}$ findings (Tatke et al., 2015) together indicates that the expression of both pcaT and $\mathrm{kgtP}$ genes is under several layers of transcriptional and post-transcriptional controls, suggesting a complex regulatory mechanism involved in modulating $P$. aeruginosa $\alpha-K G$ uptake. The presence of two $\alpha-K G$ transporters regulated in an opposite fashion in $P$. aeruginosa is of interest due to their possible function in responding to nutritional cues. Furthermore, it is also possible that the presence of two a-KG transporters is due to a difference in their substrate affinities and may indicate a role for each in changing gene expression based upon environmental $\alpha-K G$ concentrations. Future studies will determine if $P$. aeruginosa a-KG transporters are preferentially or hierarchically regulated depending on the carbon source.

In addition to the role of MifSR TCS in modulating extracellular $\alpha-K G$ transport, in this study we illustrate the interdependence of MifSR TCS and the $\mathrm{C}_{5^{-}}$ dicarboxylate $\alpha-K G$ in regulating the expression of $P$. aeruginosa's key virulence determinants. The results presented here show that MifSR TCS in an a-KG specific manner positively regulates swarming motility and pyoverdine production while negatively regulates biofilm formation and pyocyanin production. Interestingly, aKG dependent cell cytotoxicity was found to be MifS-independent and MifRdependent, suggesting a potential crosstalk between MifR and other noncognate HKs. Together, these findings strongly suggest a complex mechanism of MifSR 
action involving multiple regulatory controls that are achieved by modulating expression of numerous genes in $\alpha-K G$-dependent manner. Identifying the $\alpha-K G$ dependent members of MifSR regulon will significantly help in delineating the role of MifSR signaling pathway in $P$. aeruginosa pathogenesis.

Successful adaptation of $P$. aeruginosa to different niches depends extensively on its ability to regulate gene expression as specified by the environmental cues. Although a plethora of TCSs have been identified in $P$. aeruginosa (Winsor et al., 2011) that warrants its adaptability, only a handful of TCS signaling molecules are known to date. Recent research progresses show that, TCA cycle metabolites in-addition to their canonical role in metabolism are increasingly been recognized as a bacterial TCS activating signals (Janausch et al., 2002, Sevvana et al., 2008, Zhou et al., 2008, Brocker et al., 2009, Cai et al., 2013, Valentini \& Lapouge, 2013, Tatke et al., 2015). Our data indicates that the TCA cycle $\mathrm{C}_{5}$-dicarboxylate $\alpha-K G$ is the signaling molecule activating $P$. aeruginosa MifSR TCS. This is one of the first reports to answer fundamental questions pertaining to $\alpha-K G$ utilization in $P$. aeruginosa and its role as a messenger in MifSR regulatory mechanism. Metabolic processes and virulence mechanisms are closely interconnected and are hallmarks of $P$. aeruginosa infections. Hopefully, this and future studies on this regulatory system and metabolism pathway will add significantly to the understanding of an intricate link between virulence and metabolism in pathogens that would provide a better stratagem to fight the often indomitable infections. 


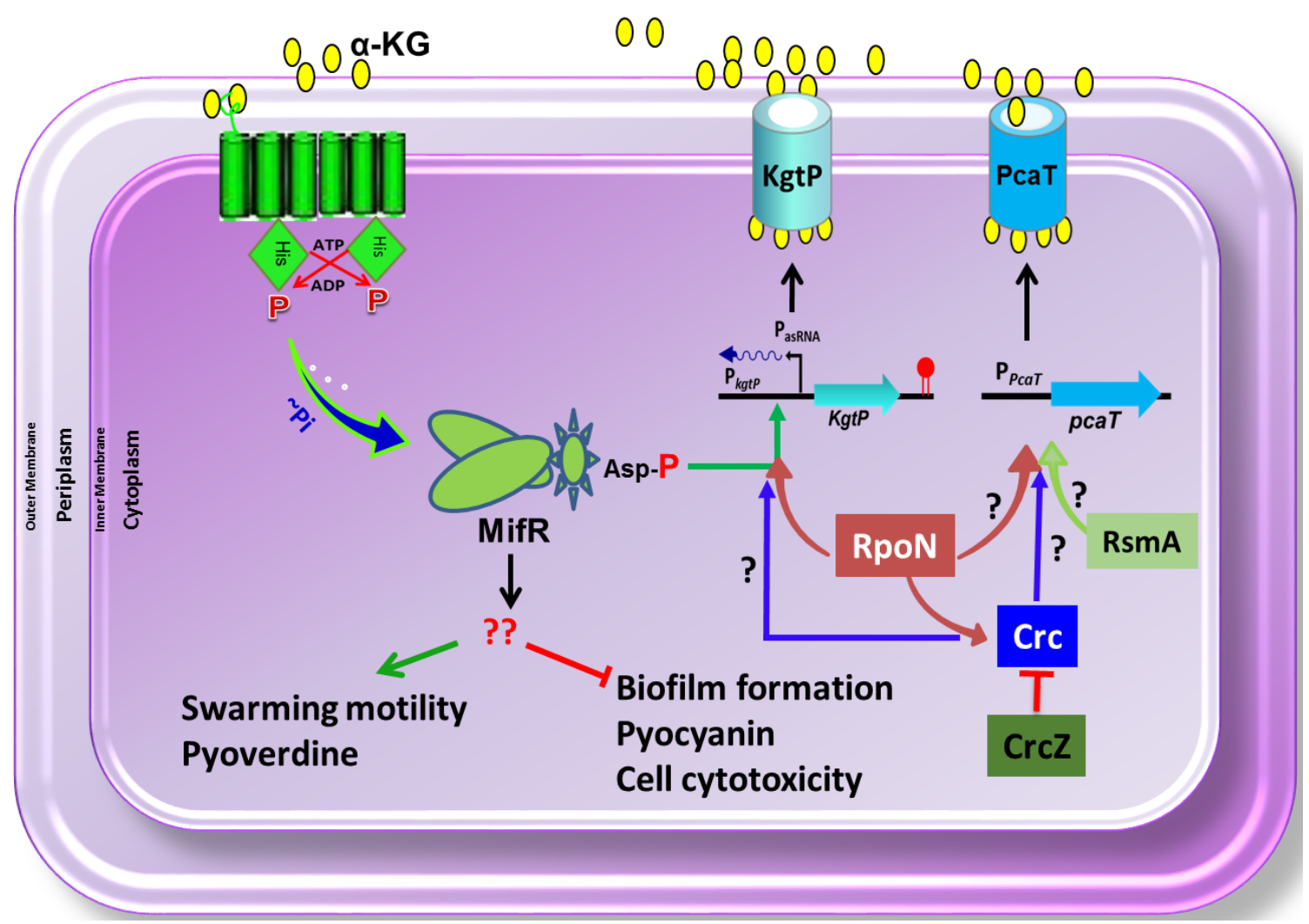

Figure 3.16: Proposed model for $\alpha-K G-d e p e n d e n t ~ M i f S$ and MifR TCS signal perception mechanism. HK-MifS senses the extracellular $\alpha-K G$ to undergo phosphorylation. The phosphate is transferred to the RR-MifR. The phosphorylated MifR in coordination with RpoN ( $\sigma 54)$ activates the expression of $\alpha-K G$ specific transporter gene PA5530. PA5530 thus enables the influx of $\alpha-K G$ to meet the metabolic and energy demands of the cells. Also, activated MifR, directly or indirectly represses the expression of pcaT gene. pcaT promoter $\left(\mathrm{P}_{\text {рсат }}\right)$ region has a $\mathrm{Crc}$ binding site, suggesting that it is under the catabolite repression control by $\mathrm{Crc} / \mathrm{CrcZ}$. The $\left(\mathrm{P}_{\text {pсaT }}\right)$ also shows the presence RsmA binding site indicating a multilayered and complex regulation of the $\alpha-K G$ transport system. Further, in the presence of $\alpha-K G$ as a carbon source MifR positively regulates swarming motility and pyoverdine production while negatively regulates biofilm formation, pyocyanin and cell cytotoxicity. 


\section{Acknowledgments}

The authors thank the following individual for their intellectual input: Hansi Kumari and Kyle Martins (Florida International University), Deepak Balasubramanian (Harvard Medical School), Lars Dietrich and Hassan Sakhtah (Columbia University)p. We would like to thank Dr. D. Haas from UNIL, Switzerland for kindly providing PA0 $\Delta r p o N$ and PA0 $\Delta r p o N:: r p o N$. We would also like to thank Dr. Rahul Mittal (University of Miami Miller School of Medicine, Miami, FL) and Dr. Philip Stahl (Washington University Medical school, St Louis, Mo) for their generous gift of the A549, a human lung adenocarcinoma epithelial cell line and the J774 Eclone, a murine macrophage cell line. This study was supported in part by the National Institutes of Health - Minority Biomedical Research Support SCORE (SC1AI081376; KM), National Science Foundation IIP-1237818 (PFI-AIR: CRESTI/UCRC-Industry Ecosystem to Pipeline Research; KM), 2013 \& 2014 Biomedical Research Initiative (BRI) Student Summer Award (NIH/NIGMS R25 GM061347; GT) and Florida International University Graduate Assistantship. The content is solely the responsibility of the authors and does not necessarily represent the official views of the funding agencies. 
CHAPTER 4

General Discussion 


\section{Overview}

Pseudomonas aeruginosa is a metabolically versatile, Gram-negative $Y$ proteobacteria that is well known for its widespread spatiotemporal distribution (Ramos, 2004). Although, a primary inhabitant of soil and water, it has an extraordinary ability to survive and thrive in a myriad of natural and artificial environments (Walker et al., 2004, Gellatly \& Hancock, 2013). Importantly, P. aeruginosa is a dominant nosocomial pathogen and an epitome of opportunistic infections in humans (Bodey et al., 1983, Lyczak et al., 2000, de Bentzmann \& Plesiat, 2011). It is the fourth most commonly-isolated nosocomial pathogen and accounts for 10 percent of all hospital-acquired infections (Gaynes et al., 2005). P. aeruginosa is infamous for its ability to cause wide range of human infections that can vary from acute to chronic, local to systemic and self-limiting to life-threatening (Campa et al., 1993, Sousa \& Pereira, 2014, Tummler et al., 2014). In particular, $P$. aeruginosa pose a high risk to individuals with impaired immunity and those suffering from the recessive genetic disorder, cystic fibrosis (Ratjen \& Doring, 2003). In patients with CF, chronic and recurrent pulmonary infections by $P$. aeruginosa are a leading cause of morbidity and mortality (Sorde et al., 2011, Winstanley et al., 2016). P. aeruginosa produces multi-determinant virulence factors, both cell associated and secretory, which modulate its pathogenicity and ensures its success as a pathogen (Balasubramanian et al., 2013). Despite extensive efforts in the development of new anti-pseudomonal drugs, therapeutic intervention in the treatment of $P$. aeruginosa infections has not been very effective, particularly due its extraordinary intrinsic and acquired resistance to a 
repertoire of clinically significant antibiotics (Hancock \& Speert, 2000, Falagas \& Bliziotis, 2007, Lister et al., 2009) . Thus, the pathogenesis of $P$. aeruginosa infections is multifactorial, and is manifested by its diverse metabolic capabilities, expression of virulence factors and antibiotic resistance.

Another key factor accounting for the success of $P$. aeruginosa as an invincible pathogen is its environmental and metabolic adaptability. This flexibility allows survival, growth and rapid colonization of different host milieus, leading to successful infection and disease progression (Silby et al., 2011). In human host, since the environmental conditions are highly variable and erratic, pathogenic bacteria need a dedicated regulatory system that supports their adaptation to perpetual changes. To perform these adaptations, $P$. aeruginosa has evolved an intricate and dynamic array of signaling networks that in addition to modulating the basic housekeeping functions, coordinate the temporal expression of various genes in response to both inter- and intracellular signaling molecules and environmental cues (Rodrigue et al., 2000, Ventre et al., 2006, Gooderham \& Hancock, 2009, Williams \& Camara, 2009, Haussler, 2010, Jimenez et al., 2012). Of the many signaling mechanisms elucidated in $P$. aeruginosa, two-component system (TCS) mediated signal perception play a pivotal role in sensing diverse environmental stimuli and elicit a pertinent and rapid adaptive response (Rodrigue et al., 2000, Stock et al., 2000, Alm et al., 2006).

TCSs in $P$. aeruginosa have been shown to regulate many physiological aspects of its lifestyle, including global responses to stress (Wood et al., 2006, Leech et al., 2008), chemotaxis (Gooderham \& Hancock, 2009), antibiotic 
resistance (Gooderham \& Hancock, 2009), carbon/nitrogen metabolism (Nishijyo et al., 2001, Li \& Lu, 2007), nutrient uptake (McPhee et al., 2006, Valentini et al., 2011, Tatke et al., 2015), expression of virulence factors (Gooderham \& Hancock, 2009, Mikkelsen et al., 2011, Okkotsu et al., 2014) and transition from free living planktonic to sessile biofilm mode of growth (Mikkelsen et al., 2011). While a plethora of TCS signaling proteins (135) have been identified in $P$. aeruginosa only a few have been extensively characterized and the regulatory targets for many TCS systems are still unknown (Rodrigue et al., 2000, Barakat et al., 2009, Winsor et al., 2011). Furthermore, in most of the cases, the environmental signals that influence TCS pathways remains obscure (Mascher et al., 2006, Mitrophanov \& Groisman, 2008). Though the environmental cues sensed by TCSs are poorly understood, they are largely accountable for determining the outcome of $P$. aeruginosa infections and pathogenesis. Also, in many instances TCSs are known to work in tandem with other signal transduction pathways, thus forming a complex network allowing $P$. aeruginosa to assimilate numerous external cues into a cohesive regulatory circuit that modulates a lifestyle continuum (Coggan \& Wolfgang, 2012). Understanding the complexities of TCS signaling is the key to understanding $P$. aeruginosa pathogenesis. 


\section{Summary}

\section{MifSR TCS regulates $P$. aeruginosa $\alpha-K G$ transport and virulence}

$P$. aeruginosa genome encodes one of the largest groups of TCS proteins identified in any sequenced bacterial species (Barakat et al., 2009, Winsor et al., 2011). Amongst these, only few TCSs have been completely characterized. This dissertation investigated the role of a previously uncharacterized TCS protein pair MifS and MifR in regulating $P$. aeruginosa pathogenesis. MifS is a sensor histidine kinase $(H K)$ and MifR is the response regulator (RR). During the course of the dissertation, the RR MifR was reported to have a dual function, in regulating the maturation stage of biofilm formation (virulence) and in pyruvate fermentation (metabolism) (Petrova \& Sauer, 2009, Petrova et al., 2012). However, the mechanism by which MifR is activated in this process remains obscure and no relation with the histidine kinase MifS has been established. Our work identified a novel role of the MifS-MifR TCS proteins in regulating $P$. aeruginosa metabolism by exclusively modulating the utilization of a key tricarboxylic acid (TCA) cycle intermediate, a-Ketoglutarate (Chapter 2) (Tatke et al., 2015). Specifically, we show that MifSR TCS regulates the uptake of extracellular $\alpha-K G$ by positively regulating the expression $P$. aeruginosa $P A 5530(\mathrm{kgtP})$ gene encoding a putative $\alpha-K G$ permease (Chapter 2, Figure 2.11). Additionally, we show that the transport of extracellular $\alpha-K G$ in $P$. aeruginosa is a consequence of an orchestrated interplay between the MifSR TCS and the alternate sigma factor RpoN $\left(\sigma^{54}\right)$ (Chapter 2, Figure 2.14). To date, this work is one of the first to establish the role of $P$. aeruginosa MifS/MifR TCS in regulates $\alpha-K G$ transport in association with the 
sigma factor RpoN (Tatke et al., 2015). In addition, we identified that besides KgtP, $P$. aeruginosa genome encodes a second $\alpha-K G$ permease PcaT (PA0229) whose expression is independent of MifSR TCS, suggesting an additional layer of regulation involved in $P$. aeruginosa $\alpha-K G$ uptake (Chapter 3). Lastly, our phenotypic assay revealed the a-KG specific exclusivity of MifSR TCS in modulating $P$. aeruginosa virulence mechanism (Chapter 3). We show that MifSR TCS in the presence of $\alpha-K G$ as a sole carbon source regulates the expression of $P$. aeruginosa biofilm formation, swarming motility, pigment production (pyocyanin and pyoverdine) and cell cytotoxicity (Chapter 3 and Figure 4.1). Simultaneous regulation of multiple mechanisms involved in $P$. aeruginosa pathogenesis suggests a complex mechanism of MifSR action. To date, to our knowledge, this is one of the first report of $\alpha-K G$ mediated two-component signaling in regulating $P$. aeruginosa metabolism and virulence. Together, this work established the role of $\alpha-K G$ as a signaling molecule and that MifSR TCS system is a master regulator of $\alpha-K G$ transport in $P$. aeruginosa. Here we propose a model for $\alpha-K G$ dependent MifSR TCS signaling in $P$. aeruginosa (Figure 4.2). 


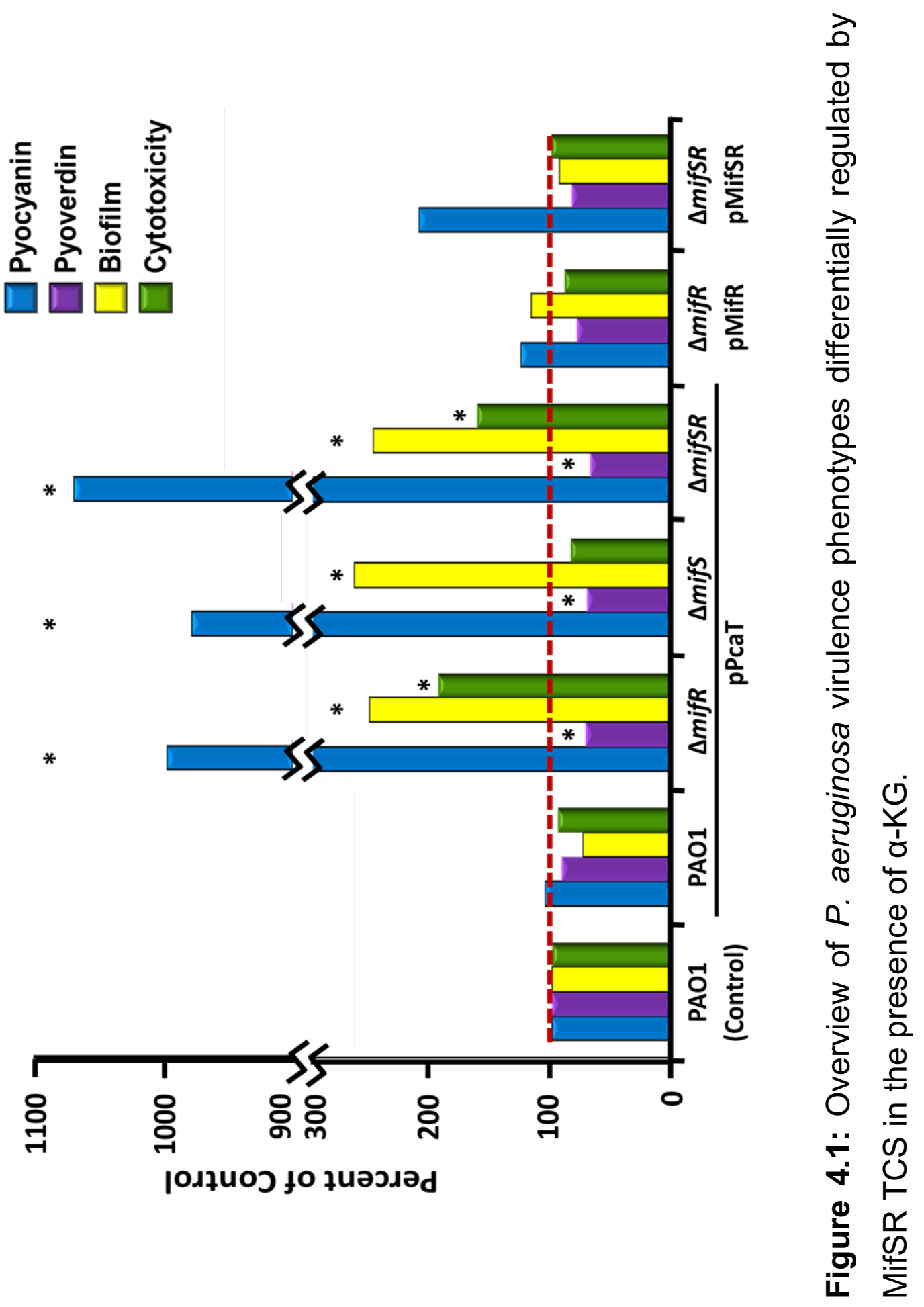




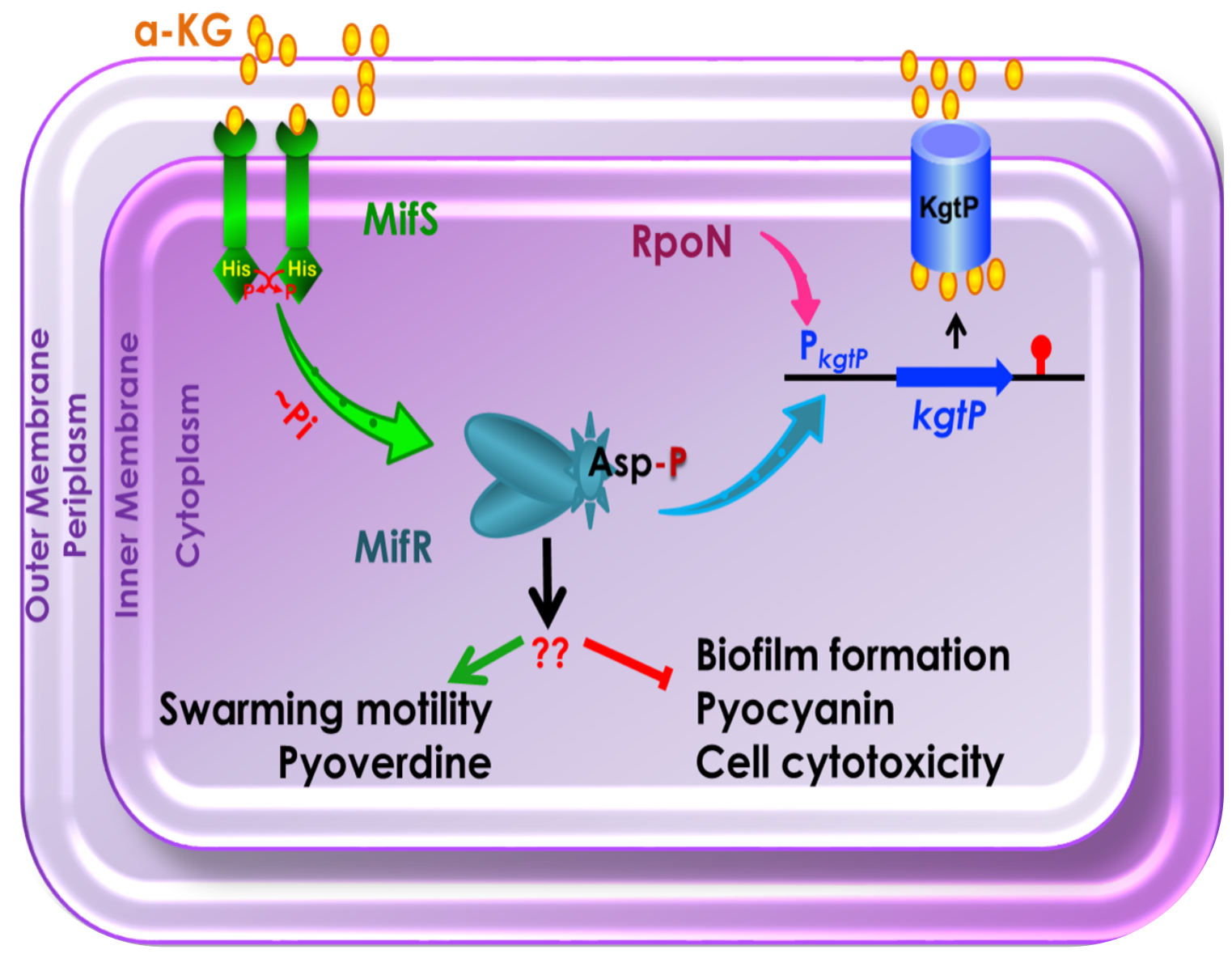

Figure 4.2: Proposed model for $\alpha-K G$ dependent MifSR TCS signaling in $\boldsymbol{P}$. aeruginosa. Sensor MifS senses the extracellular $\alpha-$ KG as an environmental cue to undergo phosphorylation. The phosphate is then transferred to the response regulator MifR. The phosphorylated MifR together with the alternate sigma factor RpoN activates the expression of $\alpha-K G$ specific transporter gene $\mathrm{kgtP}$. KgtP then enables the influx of extracellular $\alpha-K G$ to meet the metabolic demands of the cells. In addition, MifSR TCS in the presence of $\alpha-K G$ as a carbon source differentially regulates $P$. aeruginosa key virulence determinants including biofilm formation, swarming motility, cell cytotoxicity and the production of pyocyanin and pyoverdine. 


\section{Future Directions}

Two-component signal perception mechanisms that rely on phosphotransfer from histidine kinase $(H K)$ to the response regulator $(R R)$ is one of the predominant strategy used by bacteria for coupling environmental signal to adaptive responses. Bacterial TCS signal transduction mechanisms have always been a subject of extensive research because of the versatility of its components (HK and RR) to probe the environmental conditions and decide how best to respond by orchestrating the expression of several desired genes. In the recent years, with the advent of new biochemical and molecular biology techniques, substantial advances have been made in understanding the molecular mechanisms regulated by TCS signaling pathways. While the work in this dissertation using high-throughput phenotypic microarray studies and molecular and biochemical techniques have helped significantly to get an insight into a novel role of $\alpha-K G$ mediated signaling via the MifS and MifR TCS proteins in $P$. aeruginosa, there remains many unanswered questions.

Outlined below are some ideas and avenues for further research that are based on the results published in this dissertation and/or are based on my preliminary data mentioned in this section.

- Determine the $\alpha-K G$ dependent MifR regulon

- Elucidate the role of RpoN $\left(\sigma^{54}\right)$ and TCS signaling in regulating $P$. aeruginosa TCA cycle intermediate transport and pathogenesis

(Each future research direction is strengthen with a brief discussion) 


\section{Determine the $\alpha-K G$ dependent MifR regulon}

$P$. aeruginosa response regulator MifR is a part of the signal transduction MifS/MifR TCS. MifR on average is $60-65 \%$ similar to the known $P$. aeruginosa NtrC superfamily of TCS response regulators including DctD, CbrB, AauR, NtrC, AlgB, FleR and PilR, suggesting similar functions (Winsor et al., 2011). These transcriptional regulators are known to regulate a myriad of functions in $P$. aeruginosa including carbon assimilation, nitrogen metabolism, nutrient uptake, motility, antibiotic resistance (Ritchings et al., 1995, Arora et al., 1997, Gooderham \& Hancock, 2009) and alginate synthesis (Wozniak \& Ohman, 1991, Ma et al., 1998, Leech et al., 2008). Our phenotypic assays under nutrient rich conditions indicated no role of MifR in regulating $P$. aeruginosa antibiotic resistance (Figure 4.3) or other virulence related characteristics (Figure 4.4) (Tatke et al., 2015). Therefore, we performed a high throughput phenotypic microarray (BioLOG) with mifR deletion mutant and the wild-type PAO1 strains where numerous metabolic and chemical sensitivity phenotypes were tested simultaneously (Chapter 2, Figure 2.5). Our phenotypic microarray data indicated the role of RR MifR in regulating $P$. aeruginosa metabolism by modulating the utilization of $\mathrm{C}_{5^{-}}$ dicarboxylate $\alpha-K G$, a key TCA cycle intermediate as a carbon and energy source. Subsequently, our growth profile, genetic complementation and qRT-PCR expression studies showed that MifR in concert with the sigma factor RpoN $\left(\sigma^{54}\right)$ regulate extracellular $\alpha-K G$ transport by modulating the expression of $P$. aeruginosa a-KG-specific permease PA5530 (KgtP) (Chapter 2) (Tatke et al., 2015). 


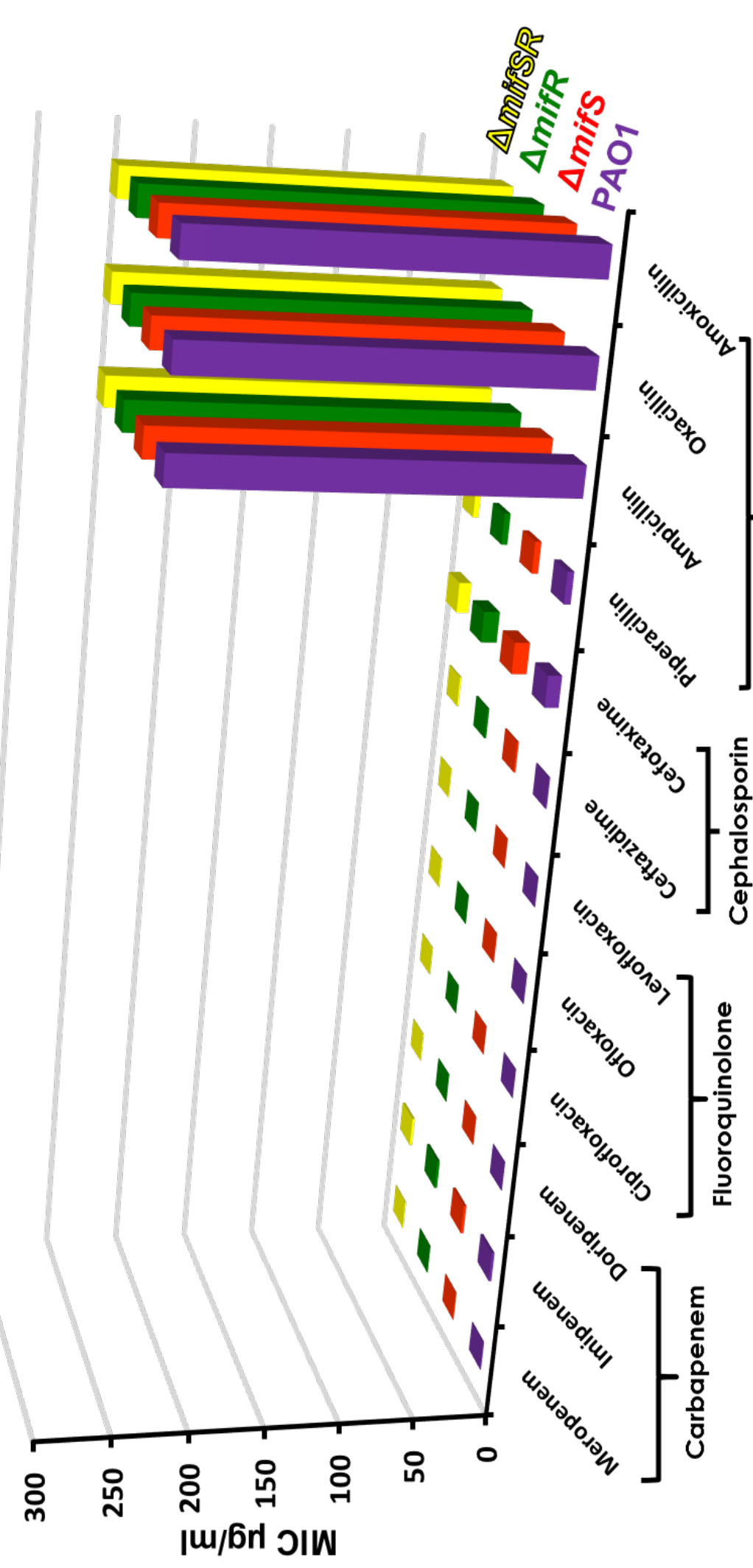

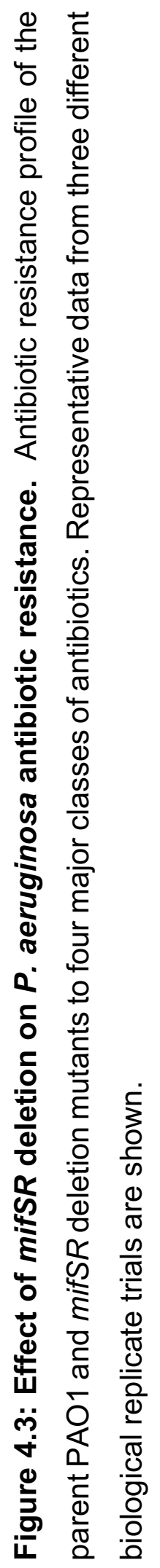




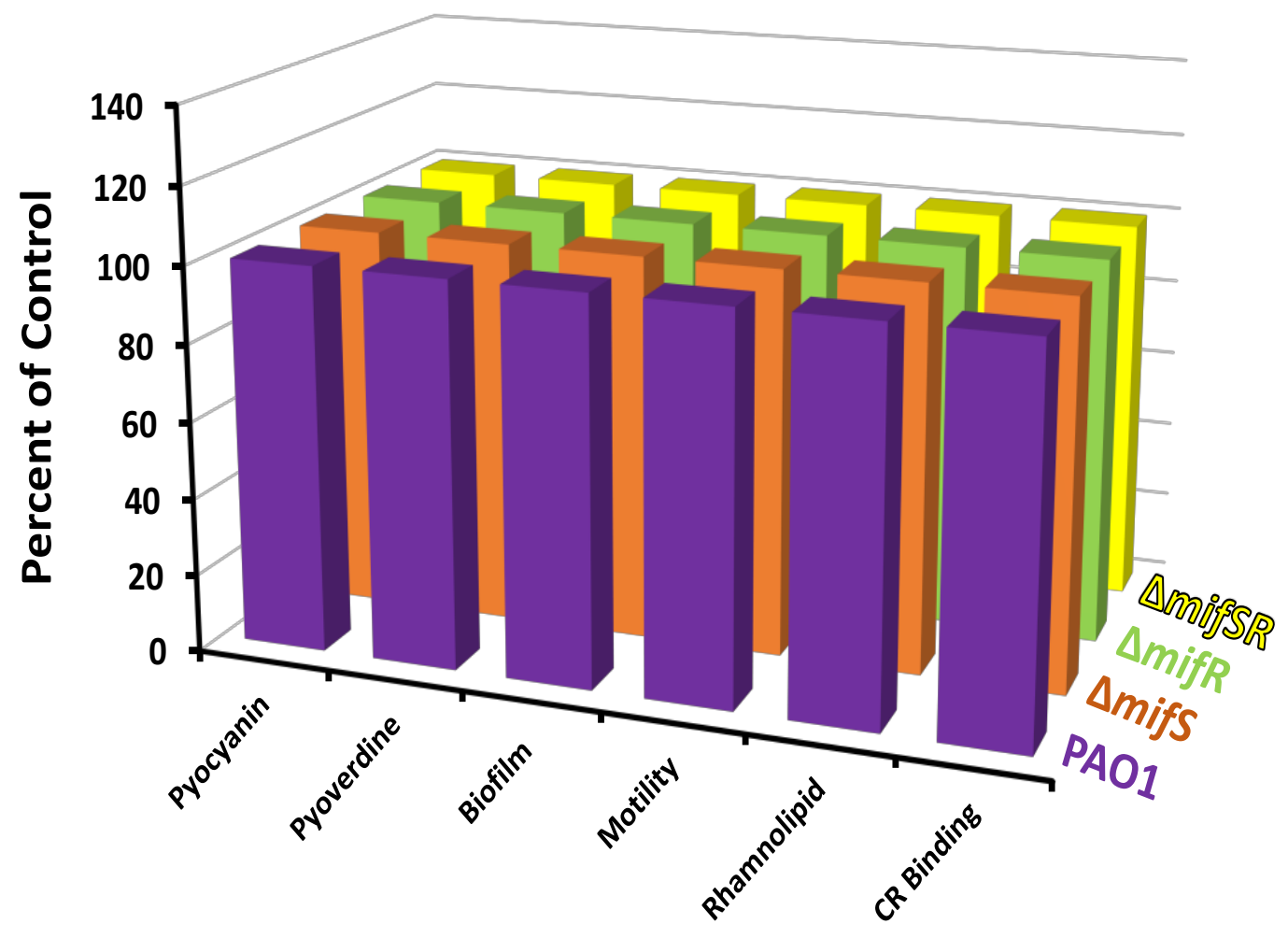

Figure 4.4: Effect of mifSR deletion on $P$. aeruginosa virulence. Overview of $P$. aeruginosa virulence phenotypes under nutrient rich conditions. 
In $P$. aeruginosa, TCA cycle intermediates, including $\alpha-K G$ is known to trigger carbon catabolite repression (CCR), a mechanism by which cells facilitate hierarchical uptake and utilization of carbon substrates for efficient growth (Wolff et al., 1991, collier 1996). Subsequent studies have showed that, CCR in Pseudomonas Spp. is mediated by the catabolite repression control (Crc) protein, a translational repressor (Rojo 2010). Our in silico analysis of the $\mathrm{kgtP}$ promoter region revealed the presence of a CA-rich motif with a signature sequence (AAc/uAAc/uAA) for the Crc protein (Chapter 2, Figure 2.13) (Tatke el al 2015). It is now well established that the expression of $\mathrm{crc}$ gene encoding the $\mathrm{Crc}$ protein is modulated by RpoN $\left(\sigma^{54}\right)$ dependent small non-coding RNA CrcZ and the CbrA/CbrB TCS (sonnleitner 2009). Also, our analyses indicates that the kgtP promoter is under a small non-coding antisense RNA (asRNA) regulation (Chapter 2, Figure 2.13) (Tatke et al., 2015). This suggests additional layers of regulation superimposed on the need for MifR in regulating $P$. aeruginosa $\alpha-K G$ transport. Subsequently, we also identified a second chromosomally encoded a-KG permease in $P$. aeruginosa namely, PA0229 (PcaT) (Chapter 3). Interestingly, our qRT-PCR expression data and complementation studies suggests that the expression of pcaT gene is independent of the MifS-MifR TCS signaling cascade. (Chapter 3, Figure 3.3). Furthermore, in silico analysis of the pcaT promoter region revealed the presence of a weak RpoN $\left(\sigma^{54}\right)$ binding site, the signature sequence (AAc/uAAc/uAA) for the translational repressor Crc protein and four putative GGA trinucleotide motifs, that are the characteristic binding sites for the posttranscriptional control RsmA family of proteins (Chapter 3, Figure 3.3). Presence, 
of shared common motifs (RpoN $\left(\sigma^{54}\right)$ and Crc binding sites) in the $k g t P$ and $p c a T$ promoter regions indicates an overlapping regulatory control. Though the role of asRNA, CrcZ, Crc and RsmA in P. aeruginosa $\alpha-K G$ transport and utilization is currently unknown, it suggest a more profound and complex mechanism of MifR controlled $\alpha-K G$ transport. This is one of the first study to report the role of a TCS response regulator in regulating bacterial $\alpha-K G$ assimilation.

Furthermore, efficient utilization and catabolism of extracellular nutrients is a primary requirement for all pathogenic bacteria to manifest their full virulence potential during the course of infection. Metabolic processes and virulence mechanisms are closely interconnected and are hallmarks of $P$. aeruginosa infections. Therefore, it is not surprising that the regulation of many virulence determinates is controlled by nutrient availability. In $P$. aeruginosa, specific carbon and nitrogen sources have been known to influence several clinically relevant virulence phenotypes such as motility (Kohler et al., 2000), pigment production (Palmer et al., 2007), biofilm development (Klausen et al., 2003(a), Shrout et al., 2006) and quorum sensing (Dandekar et al., 2012). In this study we illustrated the interdependence of RR MifR and the $C_{5}$-dicarboxylate $\alpha-K G$ in regulating the expression of $P$. aeruginosa's key virulence determinants (Chapter 3). We show that MifR in the presence of $\alpha-K G$ as the sole carbon source positively regulates swarming motility and pyoverdine production while negatively regulates biofilm formation, pyocyanin production and cell cytotoxicity (Chapter 3 and Figure 4.1). Furthermore, a previous microarray transcriptome study of the parent PAO1 and mifR deficient mutant cultivated under biofilm-specific condition exhibited 
significant alteration in the expression of genes encoding transporter proteins, secretion factors, type II and type III secretion systems (Petrova et al., 2012). Hyper pyocyanin production and increased cell cytotoxicity of the mifSR mutants suggested an overall increase in the concentration of extracellular proteins. Using the Bradford assay (Bradford, 1976) we quantitated the amount of total proteins present in the culture supernatant of the mifSR mutants grown overnight in the presence of $\alpha-K G$ and/or succinate (30 mM each), as a sole carbon source. The results obtained were intriguing as the concentrations of extracellular proteins in the culture supernatant were significant lower in the miSR mutants as compared to the wild-type PAO1 strains in the presence of $\alpha-K G$ (Figure 4.5). In addition, when cells were grown in the presence of succinate no difference was observed in the extracellular protein concentration in mifSR mutants compared to the wildtype PAO1 (Figure 4.5). Together, this data indicates that MifR in the presence of a-KG affects numerous transport and virulence associated mechanisms in $P$. aeruginosa. However, at this point, the transcription regulatory network and the gene expression pattern regulated by MifR that manifest these phenotypic effects are unclear.

There are numerous previously described signaling pathways in $P$. aeruginosa that independently or in association are capable of positively regulating swarming motility and negatively regulating biofilm formation and pyocyanin production. These pathways include the cell density dependent quorum sensing (Las, Rhl and PQS) signaling system, GacS/GacA two-component system and cyclic-di-GMP signaling (Goodman et al., 2004, Caiazza et al., 2007, Kuchma et 
al., 2007, Merritt et al., 2007, Merritt et al., 2010). In a recent study, Pertova and co-workers reported that, mifR overexpression in the $P$. aeruginosa parent PAO1 strain positively affects cyclic-di-GMP levels in a biofilm-specific manner (Petrova et al., 2012). At this point it is not clear if any of these pathways are regulated by MifR in the presence of $\alpha-K G$, either directly or indirectly. Future work focused on elucidating the $\alpha-K G$ dependent members of MifR regulon using the high throughput RNA-seq approach will provide a new perspective to decode and better understand the correlations between $P$. aeruginosa metabolism and virulence. These studies will also substantially impact our understanding of the profound role played by the metabolite $\alpha-K G$ as a messenger in regulating molecular pathogenesis of the intractable pathogen $P$. aeruginosa. I have finished the required RNA-Seq experiments using the wild-type PAO1 and PAODmifR mutant derivatives expressing pcaT in trans in the presence and absence of $\alpha-K G$. The RNA-seq was performed using the Next-generation sequencing Illumina Platform and the RAW FASTQ data has been generated. Currently, I am analyzing and validating the RNA-seq data using CLC Genomic Workbench, Python programming and qRT-PCR expression studies. Future work focused on validation of RNA-seq data using phenotypic assays will help to better understand the role of RR MifR and the metabolite $\alpha-K G$ in regulating $P$. aeruginosa physiology. 


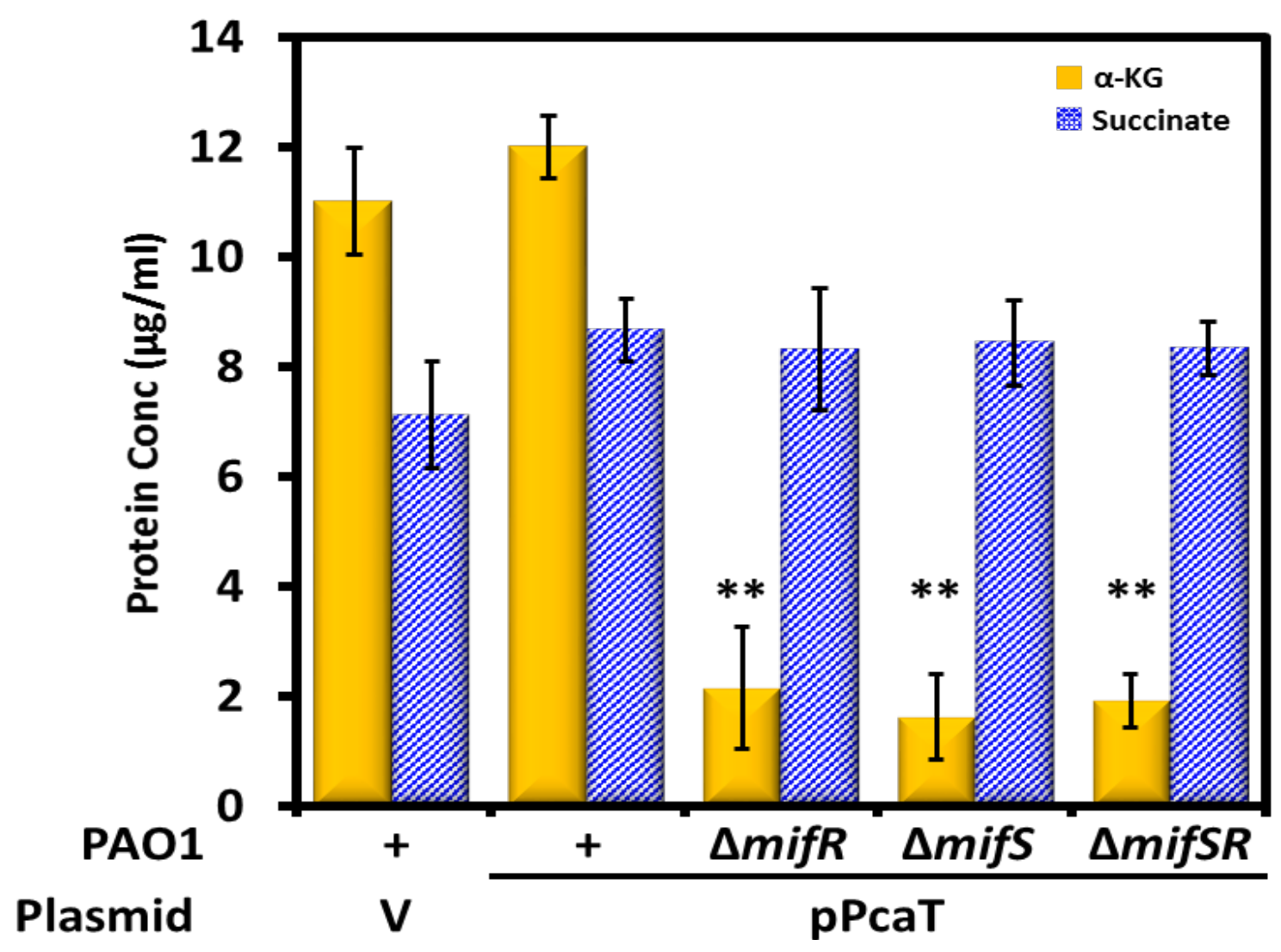

Figure 4.5: Effect of mifSR deletion on extracellular protein concentration. The extracellular proteins were quantitated using the Bradford assay from the culture supernatant of mifSR mutants grown over night in the presence of $\alpha-K G$ and succinate ( $30 \mathrm{mM}$ each), as the sole carbon source. 


\section{Elucidate the role of $\operatorname{RpoN}\left(\sigma^{54}\right)$ and TCS signaling in regulating $P$. aeruginosa TCA cycle intermediate transport and pathogenesis}

Success of $P$. aeruginosa as a recalcitrant pathogen can be largely attributed to the repertoire of transcriptional regulators encoded by its genome. These regulators form an intricate and dynamic networks to exquisitely tune the expression of genes responsible for phenotypic adaptation and virulence during infection. $P$. aeruginosa has one of the largest genome amongst sequenced bacteria and transcriptional regulators encompass about $10 \%$ of the genome (Galan-Vasquez et al., 2011). Amongst transcriptional regulators, sigma ( $\sigma$ ) factors are the indispensable global regulators of gene expression. They confer promoter recognition specificity to the RNA polymerase core enzyme (Borukhov \& Severinov, 2002, Campbell et al., 2002, Murakami \& Darst, 2003) in order to orchestrate the process of transcription initiation (Burgess et al., 1969), a key step in gene regulation (Browning \& Busby, 2004). To date, the regulatory machinery predicted in $P$. aeruginosa prototypic strain PAO1 comprises of approximately 550 transcriptional regulators and 26 putative sigma factors (one RpoN $\left(\sigma^{54}\right)$, eight $\operatorname{RpoD}\left(\sigma^{70}\right)$, and 17 of extra cytoplasmic function (ECF) family (Potvin et al., 2008, Perez-Rueda et al., 2009, Galan-Vasquez et al., 2011). Amongst these of special interest is the alternate sigma factor $\mathrm{RpoN}\left(\sigma^{54}\right)$ which is a global regulator involved in $P$. aeruginosa nitrogen metabolism, motility, carbon assimilation, nutrient transport, quorum sensing and virulence (Potvin et al., 2008).

Our comparative growth curve analyses of $P$. aeruginosa wild-type PAO1 and the PAO $\triangle r p o N$ mutant strains in the presence of TCA cycle $\mathrm{C}_{4}, \mathrm{C}_{5}$ and $\mathrm{C}_{6}$ di- 
and tricarboxylic acid intermediates exhibited intriguing results (Figure 4.6). The PAOArpoN mutant failed to grow in the presence of all the TCA cycle intermediates including succinate $\left(\mathrm{C}_{4}\right)$, $\alpha-K G\left(\mathrm{C}_{5}\right)$ and citrate $\left(\mathrm{C}_{6}\right)$ as the sole carbon source (Figure 4.6). Furthermore, the growth defect exhibited by PAO $\triangle r p o N$ could be restored to the parent strain PAO1 levels by introducing the wild-type $r p o N$ in the $\mathrm{PAO} \Delta r p o N$ mutant (Figure 4.6). This data strongly hints at the role of RpoN $\left(\sigma^{54}\right)$ as the master regulator of TCA cycle $\mathrm{C}_{4}, \mathrm{C}_{5}$ and $\mathrm{C}_{6}$ di- and tricarboxylic acid intermediate transport in $P$. aeruginosa. A unique feature of the $\operatorname{RpoN}\left(\sigma^{54}\right)$ dependent transcription activation of genes is the requirement of an additional transcriptional regulatory protein called the $\mathrm{RpoN}\left(\sigma^{54}\right)$ binding protein that aids in transcription initiation (Thony \& Hennecke, 1989, Potvin et al., 2008). In P. aeruginosa, to date, $22 \mathrm{RpoN}\left(\sigma^{54}\right)$ binding proteins have been predicted of which nine (close to $41 \%$ ) belong to the signal transduction TCS. In the extensively studied Rhizobium meliloti and Rhizobium leguminosarum, RpoN $\left(\sigma^{54}\right)$ together with the RR DctD, a part of the DctB/DctD TCS pair, is reported to regulate the transport of $\mathrm{C}_{4}$-dicarboxylates succinate, fumarate and malate (Ronson et al., 1987, Watson, 1990, Janausch et al., 2002).

In $P$. aeruginosa, three TCS protein pairs namely, DctB/DctD, MifS/MifR and PA1336/PA1335 have been identified to be homologous to the Rhizobium RpoN dependent $\mathrm{C}_{4}$-dicarboxylate transport regulatory DctB/DctD TCS (Tatke et al., 2015). The response regulator proteins of these TCS pairs namely, DctD, MifR and PA1335 share $67 \%, 69 \%$ and $68 \%$ identity to the $R$. meliloti RR DctD (Winsor et al., 2011), suggesting a similar function. During the course of the dissertation, 
Valentini et al. showed that, $P$. aeruginosa DctD in concert with $\operatorname{RpoN}\left(\sigma^{54}\right)$ regulates the transport of $\mathrm{C}_{4}$-dicarboxylate succinate, fumarate and malate by initiating the transcription of $\operatorname{dct} A$ and $\operatorname{dctPQM}$ genes encoding the $\mathrm{C}_{4^{-}}$ dicarboxylate transporters (Valentini et al., 2011). Also, we recently reported a key finding that the assimilation of extracellular $C_{5}$-dicarboxylate $\alpha-K G$ requires a functional RpoN $\left(\sigma^{54}\right)$ to work in tandem with the RR MifR in order to activate the expression of $P$. aeruginosa $\alpha-K G$ permease encoding $k g t P$ (PA5530) gene (Chapter 2) (Tatke et al., 2015). Interestingly, P. aeruginosa RRs DctD and MifR are functionally exclusive with the only shared feature being the requirement of RpoN (Valentini et al., 2011, Tatke et al., 2015). This suggests that $P$. aeruginosa has a dedicated sensory-response mechanism to facilitate the assimilation of specific class of TCA cycle intermediates $\left(\mathrm{C}_{4}, \mathrm{C}_{5}\right.$ and $\left.\mathrm{C}_{6}\right)$, respectively. While the role of $P$. aeruginosa RpoN in the transport of $\mathrm{C}_{4}$ (succinate, fumarate, malate) (Valentini et al., 2011) and $C_{5}$ di-carboxylates ( $\left.\alpha-K G\right)$ (Tatke et al., 2015) is reported, its role in the transport of $\mathrm{C}_{6}$-tricarboxylate (citrate) is unclear. Also, the role of PA1336/PA1335 TCS in $P$. aeruginosa is unknown.

Our sequence analysis of $P$. aeruginosa PAO1 genome revealed the presence of two ORFs PA5476 (citA) and PA5468 encoding the putative $\mathrm{C}_{6}{ }^{-}$ tricarboxylate citrate transport proteins (Winsor et al., 2011). The promoter for citA and PA5468 genes have the putative $-12 /-24$ RpoN $\left(\sigma^{54}\right)$ binding signature sequences (Thony \& Hennecke, 1989, Winsor et al., 2011). Furthermore, the RR PA1335 has the conserved domains found among NtrC family of regulators, an Nterminal regulatory, a central RpoN $\left(\sigma^{54}\right)$ activation and a C-terminal DNA binding 
domains (Winsor et al., 2011). Since PA1335 exhibits high identity to RpoN $\left(\sigma^{54}\right)$ dependent transcriptional regulators, it is possible that the RR PA1335, part of the PA1336/1335 TCS in concert with RpoN facilitate extracellular citrate transport by regulating the expression of citA and PA5468 genes. Future studies aimed at characterizing the role of RpoN and PA1336/PA1335 TCS in citrate transport will provide a vital missing link in our understanding of the fundamental aspects of $P$. aeruginosa di- and tri carboxylate transport (Figure 4.7).

It is worth mentioning that $P$. aeruginosa utilizes TCA cycle intermediates as the preferred carbon, nitrogen and energy source (Wolff et al., 1991, Collier et al., 1996). The involvement of RpoN $\left(\sigma^{54}\right)$ in regulating the uptake of TCA cycle intermediates strongly suggests its role as a protagonist that decides the fate of carbon and energy utilization patterns in P. aeruginosa. Recently, amongst all sigma factors, RpoN has been recognized as a central player having the largest impact on $P$. aeruginosa global gene expression that ensures its pathogenic success (Schulz et al., 2015). Future studies using comparative transcriptome profiling approaches should elucidate the function-specific molecular mechanisms manifested by RpoN in concert with DctB/DctD, MifS/MifR and PA1336/PA1335 TCS regulatory proteins. Furthermore, it would be interesting to determine if RpoN and the DctB/DctD, MifS/MifR and PA1336/PA1335 function together in any capacity to regulate $P$. aeruginosa virulence in a nutrient source specific manner. These studies will significantly contribute to a greater understanding of $P$. aeruginosa's complex metabolic networks and will provide a reliable scaffold for elucidating the transcriptional network of this portentous pathogen. 


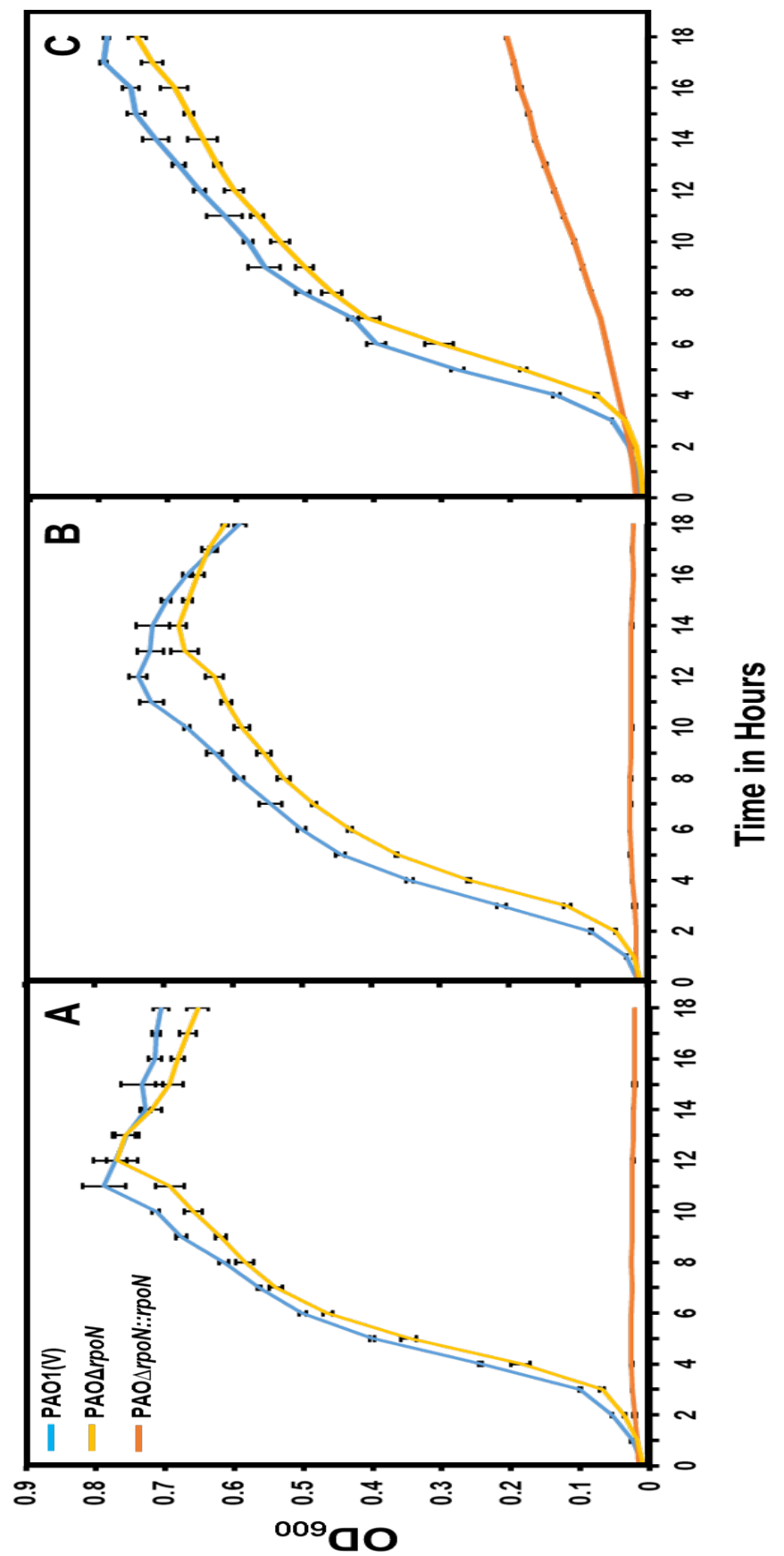

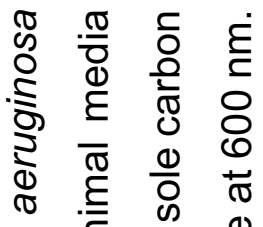

¿

( )

\.

달

गี

म

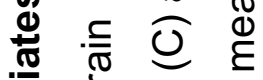

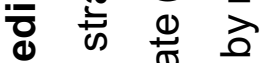

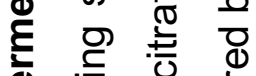

志

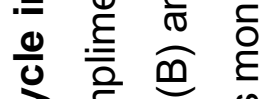

उे है

$\varangle$ 苋莡

일 岳

पㄴำ क ठำ

U

ป

ఏ \寸

व 1 \& 0

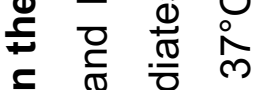

$\leq$ ते

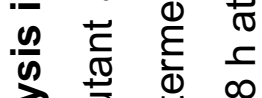

त)

沗 $之 \frac{0}{0}$

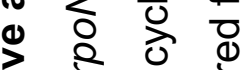

ป ป

ᄃ

ป

$\ddot{0}$ ○

ச்

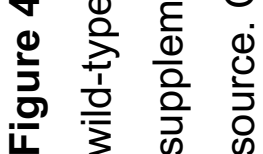




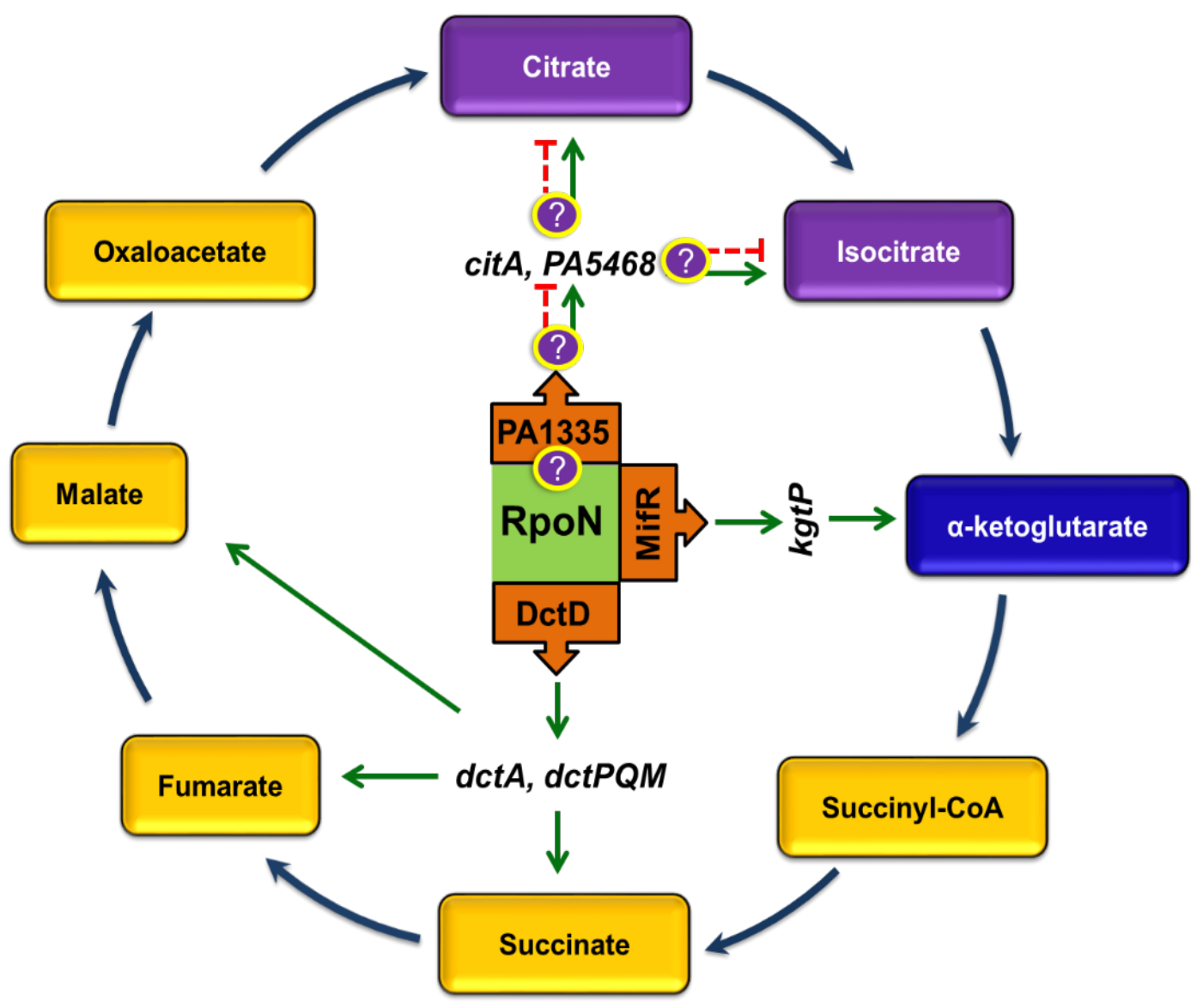

Figure 4.7: $P$. aeruginosa RpoN and TCS mediated TCA cycle intermediate transport. Model representing $P$. aeruginosa RpoN and TCS response regulator mediated transport mechanism of TCA cycle $\mathrm{C}_{4}$ (yellow), $\mathrm{C}_{5}$ (blue) and $\mathrm{C}_{6}$ (purple) di- and tri-carboxylates. The response regulators are represented in orange. Green and red arrows represent positive regulation 


\section{Concluding Remarks}

Due to the rapid emergence of antibiotic resistance in bacterial pathogens, researchers are relentless search for new targets for drug discovery. Although, TCSs have been identified in lower eukaryotes such as yeast and plants, no reports on the presence of TCS in mammalian cells have been reported (Gotoh et al., 2010). This has made TCS proteins an attractive drug target (Schreiber et al., 2009, Gotoh et al., 2010, Bem et al., 2015). Furthermore, the metabolite $\alpha-K G$ itself has been considered to have significant therapeutic potential in mammals (Grzesiak et al., 2016, Zdzisinska et al., 2016). Our results indicates that $\alpha-K G$ is a repressor of $P$. aeruginosa acute (pyocyanin and cell cytotoxicity) and chronic infection (biofilm formation) phenotypes. Understanding the physiological effects of $\alpha-K G$ on $P$. aeruginosa and its effect manifested through MifR transcriptional control will help significantly in development of new therapeutic options. 
APPENDICES 


\section{Appendix 1}

\section{$70^{\text {th }}$ Anniversary Collection of the Society for General Microbiology: Journal of Medical Microbiology}

This chapter has been published:

Kalai Mathee, Lynn L. Silver, and Gorakh Tatke.

2015. J Med Microbiol. 64(12):1457-61

DOI: $10.1099 / \mathrm{jmm} \cdot 0.000186$ 
Abstract

In the last 70 years, we have seen a radical change in our perception and understanding of the microbial world. During this period, we learned from Woese and Fox there exists a third kingdom called "Archea" based on the phylogenetic studies of the 16S ribosomal RNA that revolutionized microbiology (Woese \& Fox, 1977, Woese et al., 1978). Further, we were forced to reckon with the fact that Koch and Pasteur's way of growing cells in test-tubes or flasks planktonically does not necessarily translate to the real-life scenario of bacterial life style, where they prefer to live and function as a closely knit microbial community called biofilm. Thanks are due to Costerton, who led the crusade on the concept of biofilms and expanded its scope of inquiry, which forced scientists and clinicians worldwide to rethink how we evaluate and apply the data. Then progressively, disbelief turned into belief, and now it is universally accepted that the micro-organisms hobnob with the members of their community to communicate and coordinate their behavior, especially in regards to growth patterns and virulence traits via signaling molecules. Just when we thought that we were losing the battle against bacteria, antimicrobials were discovered. We then witnessed the rise and fall of antibiotics and development of antibiotic resistance. Due to space and choice limitation, we will focus on the three areas that caused this major paradigm shift (i) antimicrobial resistance (AMR), (ii) biofilm and (iii) quorum sensing (QS), and how the Journal of Medical Microbiology played a major role in advancing the shift. 


\section{Antimicrobial resistance (AMR)}

It is notable that this 70 th anniversary collection for the journal coincides with the 70th anniversary of the Nobel Prize to Florey and Chain for their work on penicillin, a $\beta$-lactam antibiotic. It was first used to treat a patient in 1942 , which revolutionized medicine and ushered in the age of antibiotics. Yet, resistance mechanisms that could inactivate penicillin, the $\beta$-lactamases, were already present among bacteria (Abraham \& Chain, 1988). As has been uncovered over the years, the origins of most drug-specific antibiotic resistance mechanisms are genetically encoded functions that predate the introduction of the antibiotics. These mechanisms have been derived from the antibiotic-producing organisms, from the pathogens themselves or from commensals or environmental bacteria. Selective pressure by antibiotics has led to horizontal transmission of these resistance genes and further optimization (evolution) of the mechanisms (Davies, 1994, Davies \& Davies, 2010).

The Journal of Medical Microbiology has published many insightful reviews on AMR, and important laboratory and epidemiological studies of the prevalence and spread of specific resistance mechanisms. A 1973 paper (Anderson et al., 1973) presented a study of antibiotic-resistance transfer in the intestines of human

volunteers, finding that Resistance-factors (R-factors) were successfully transferred when treated with antibiotics, and not in the absence of treatment. A later review of plasmid-mediated, horizontally-transmitted, enzymatic resistance to ampicillin and trimethoprim was the subject of an Oakley Lecture (Amyes, 1989). This lecture acknowledged the growing numbers of resistance determinants to 
each antibiotic, but emphasized evolutionary patterns showing that the enzymes fell into a small number of groups. The metallo- $\beta$-lactamases, which can hydrolyze the carbapenems, were flagged more than 20 years ago as an emerging medical challenge (Payne, 1993). That challenge was realized recently with the advent of the NDM-1 metallo- $\beta$-lactamase. A recent review uses the rapid global spread of the NDM-1 $\beta$-lactamase as an example - and a warning - of the looming public health problem of antibiotic resistance that may only be controlled through international cooperation (Johnson \& Woodford, 2013).

It is not only transmissible factors that are responsible for AMR. Especially in Gram-negative pathogens, intrinsic impermeability of the cell envelope and efflux mechanisms also plays a role. Mutational changes in these factors can lead to decreased susceptibility. Pseudomonas aeruginosa is a particularly problematic pathogen because it utilizes a variety of these mechanisms to put up a formidable barrier, limiting greatly the number of classes of chemotherapeutics that can overcome those mechanisms, to $\beta$-lactams, aminoglycosides, fluoroquinolones and colistin. A recent review in the Journal of Medical Microbiology describes this phenomenon (Strateva \& Yordanov, 2009). In addition to permeability barriers and efflux, certain antibiotics, such as fluoroquinolones, can select mutations in the genes encoding their molecular targets. For the commonly used systemic monotherapeutic agents, resistance does not occur via single mutations in the target as they are generally not single proteins but are, rather, the products of multiple genes or a pathway (Silver, 2007). It is clear that new antimicrobial therapies are needed to bolster the diminishing antibacterial armamentarium, but 
this is a difficult undertaking (Silver, 2011). AMR has become a real and proximate threat that requires constant monitoring - and the Journal of Medical Microbiology remains at the forefront of the undertaking.

\section{Biofilm}

The notion that microorganisms, in nature, exist as a sessile, systematized, multicellular communities was first described in the late $17^{\text {th }}$ century by Van Leeuwenhoek. He examined plaque samples from his own teeth and found them to be a complex aggregation of micro-organisms, which he then referred to as "animalcules". Even though the biofilm mode of bacterial existence was known for several centuries, the concept was not promulgated until the late $20^{\text {th }}$ century. The late 1980s and 1990s saw an increased recognition of the fact that bacteria in their natural habitat survive and function as organized communities, encapsulated within a polymeric matrix - a crusade led by Costerton, the pioneer and father of biofilm research (Costerton et al., 1987, Lappin-Scott et al., 2014).

In the years that followed, $P$. aeruginosa emerged as the model organism to glean information on the various aspects of biofilm mode of bacterial lifestyle. Our appreciation for this lifestyle was facilitated by the advent of non-destructive investigation techniques, especially the use of live-monitor systems with confocal laser scanning microscopy. This provided an unprecedented ability to see (and almost feel) the beautiful architectural formations held together by an extracellular matrix. This also led us to question many aspects of biofilm that were accepted as a fact, especially where repetition has led to persuasion - the illusory truth effect. At that point, it was well established that $P$. aeruginosa secretes alginate, a 
complex exopolysaccharide made of guluronic and mannuronic acid that provides selective advantage for its survival in lungs of patients with cystic fibrosis (Song et al., 2003). The scientific community was persuaded that alginate was the matrix in P. aeruginosa biofilms. The Journal of Medical Microbiology was bold enough to publish the paper by Stapper et al that questioned the "illusion of truth" (Stapper et al., 2004). Notwithstanding the importance of alginate in infection, this paper alluded to the potential contribution of other polysaccharides, which turned out to be Psl and Pel polysaccharides (Ma et al., 2012, Jennings et al., 2015).

Micro-organisms coexist in a complex society that includes bacteria, fungi, archaea and viruses, forming the multifaceted polymicrobial or mixed species biofilm communities. This provides the communities a competitive advantage such as AMR, metabolic cooperation, quorum sensing (QS), and many other synergies. It is only in the last few years that we have begun to fathom the complex nature of the mixed-species biofilm phenotype and its physiological role during infection. Adam et al presented a study on mixed fungal-bacterial biofilms using Candida albicans and Staphylococcus epidermidis, organisms that are frequently implicated in catheter-associated infections (Adam et al., 2002). Their findings highlighted the coexistence of different microbial species, and their interdependence in the biofilm formation and AMR. In a follow-up study, the same group focused on the composition of Candida's polymeric matrix, which contains carbohydrates, proteins, hexosamine, phosphorous and uronic acid, and its importance in promoting AMR (Al-Fattani \& Douglas, 2006). Their work provided a critical insight into the complex biofilm phenotype. Following a series of articles in 
the journal, Wilson published an insightful review that highlighted the antimicrobial sensitivity in oral biofilms (Wilson, 1996). This review summarized studies pertaining to the susceptibility of oral bacteria to antimicrobials, based on the type of biofilm models used. The author also emphasized the need for research in studying the resistance profile of oral biofilm communities, which can be crucial in developing an efficient therapeutic regimen. The Journal of Medical Microbiology has been instrumental in advancing biofilm research by publishing these studies, first of their kind to focus on the mixed-species biofilm and its contribution to AMR.

More importantly, the recognition of mixed biofilms in nature was the precursor to the microbiome studies that were facilitated by metagenomic analyses. Now, one could look at the potential relationship between groups of organisms within a single niche that makes a bacterial social network (Fernandez et al., 2015). This was clearly facilitated by metagenomic analysis heralded by polymerase chain reaction (PCR) that has become a breastwork in all laboratories, replacing traditional techniques. This has revolutionized how we view ourselves, realizing that the number of bacteria exceeds the number of human cells by 10:1. The ease with which DNA is being sequenced and the rapid growth of DNA databases, and the development of eubacterial-specific, fungal-specific and species-specific primers, will enable rapid detection of micro-organisms that will become a mainstay in clinical laboratories. Although we borrowed the knowledge of soil microbiologists who developed bacterial-specific primers over 20 years ago, we have to thank the human microbiome project (HMP) launched in 1990, which truly contributed to the refinement of many bacterial-specific DNA primers (Jaric et 
al., 2013). The Journal of Medical Microbiology contributed to this revolution by publishing two articles, one that focuses on fungal-specific (Makimura et al., 1994) and the other on bacterial-specific (Harris \& Hartley, 2003) analyses. The latter compared molecular diagnostics with standard culture techniques and offers a broad-range 16S rDNA PCR optimized to obtain the highest level of sensitivity for the detection of bacteria in clinical specimens. The future of rapid detection and diagnosis of infection truly rests on adopting molecular tools as part of routine clinical work.

The HMP studies have contributed significantly to our knowledge of the human microbiome (Turnbaugh et al., 2007). It is now known that the human gastrointestinal tract alone harbors a diverse array of microbes that are critical to host nutrition, regulation of intestinal angiogenesis, and development of the immune response. Alterations in the composition of the intestinal microbiota have been associated with various disease states (Larson \& Welch, 1993, Masseret et al., 2001). A leading example of this is Clostridium difficile-associated diarrhea (CDAD), an infection caused by the Gram-positive $C$. difficile. CDAD occurs primarily in patients whose colonic microbiotas have been functionally altered by antibiotic therapy (Kelly \& LaMont, 2008). C. difficile was identified in 1935 in a stool sample from a healthy neonate (Hall \& O'Toole, 1935). However, it was not until 1976, just over four decades after its identification, that it was successfully cultured using the reinforced clostridial medium (Hafiz \& Oakley, 1976). The Journal of Medical Microbiology took the lead in publishing the findings at a time when $C$. difficile was still considered a human commensal and was not associated 
with any disease. It was later in 1978 that $C$. difficile was first reported to be a human pathogen (Larson et al., 1978). In the recent years CDAD has become rampant, with morbidity and mortality increased over the decades past (Kelly \& LaMont, 2008, Britton \& Young, 2014). Colonic dysbiosis due to the use of antibiotics favors $C$. difficile germination and growth, as it is resistant to most of the antibiotics (Knecht et al., 2014, Theriot et al., 2014). Following the HMP, many studies have targeted alternative therapeutic regimens against C. difficile, including fecal transplantation. The Journal of Medical Microbiology has always been in the vanguard even before the HMP published data pertaining to the fecal microbial load (Stephen \& Cummings, 1980) and the effect of $C$. difficile infection on fecal microbiome (Hopkins \& Macfarlane, 2002).

\section{Quorum sensing (QS)}

In the 1960 s and 70 s, scientists decried the notion that bacteria have the ability to communicate. In the 1990 s this notion was christened as QS, by which bacteria can sense bacterial numbers (cell density), integrate and process the environmental cues, and coordinately modify their behavior by expressing target genes (Nealson \& Hastings, 1979, Greenberg, 2003). Now, ample evidence exists for inter-species, inter-genera and inter-kingdom communications using largely diffusible small molecules called quoromones or autoinducers (Williams, 2007). In Gram negative, these molecules are largely made of small chemicals, whereas Gram positive tend to use oligopeptides. These signaling molecules interact with cognate receptors. It became clear that bacteria employ QS to coordinate the expression of virulence factors critical for initiation and establishment of infection. 
It was also shown that the QS molecules themselves acted as virulence factors by being potent stimulators of multiple eukaryotic cells (Smith \& Iglewski, 2003). Subsequently, a flurry of activities began in this area with everyone looking to link their pet system to QS. A significant publication in Science using the $P$. aeruginosa PAO1, which had seen many passages and its isogenic QS mutants, argued the complete dependence on QS for biofilm formation (Davies et al., 1998). Once again, the Journal of Medical Microbiology led the way in debunking this story. In 2007, Schaber and colleagues were able to isolate QS-deficient clinical isolates and demonstrate their ability to form biofilms, although the quality of the biofilm varies (Schaber et al., 2007). This seriously questioned whether QS was critical for biofilm formation and infection. In fact, recent work by Greenberg's team showed that within a colonizing community, one could have social cheaters who can coexist and that QS plays a role in policing this cooperation (Wang et al., 2015).

With the emergence of AMR it seems natural that research turn towards finding anti-QS molecules as an alternative therapy. With the first report of furanones as anti-QS molecules (Manefield et al., 1999), there was a slew of articles that focused on everything from natural products to synthetic compounds. Once again, the opportunistic pathogen $P$. aeruginosa became the protagonist in the search for anti-QS molecules, but investigations also included Gram-positives such as Staphylococcus spp. (Nostro et al., 2007). Some researchers randomly looked at herbs, including ancient remedies such as ginseng (Schneper et al., 2011), and others took an ethnobotanic approach by looking at natural products 
that are used by local communities for medicinal value (Adonizio et al., 2006). The latter identified various medicinal plants that had anti-QS activities which attenuated bacterial pathogenic virulence factor production, and could rescue Caenorhabditis elegans from P. aeruginosa-induced death (Adonizio et al., 2008). However, with over 15 years of searching, an anti-QS panacea has not emerged. This is, in part, due to the ability of the QS and the anti-QS molecules to elicit an immune response that can lead to inflammation.

Though the focus has largely been on looking at the infecting organism for drug targets, we tend to forget that the host contributes to the bacterial shenanigans. It was well known that in the lungs of cystic fibrosis patients, $P$. aeruginosa lives in a novel biofilm lifestyle as aggregates (Sriramulu et al., 2005) surrounded by "frustrated" phagocytes (Høiby et al., 1993), and accumulate mutations (Oliver et al., 2000). However, it was in the late 1990s that the role of neutrophils in the critical phenotypic conversion contributing to poor prognosis for the patient was demonstrated (Mathee et al., 1999). This study suggested antioxidants and anti-inflammatory agents as potential therapeutic measures. In 2009, a paper by Parks and colleagues not only explored the role of neutrophil in the quality and quantity of biofilm, but also proposed neutrophil necrosis as a potential drug target (Parks et al., 2009).

In this 70 year journey, the Journal of Medical Microbiology has been one of the torch bearers in advancing scientific research by critically reviewing and publishing articles related to the diverse medical microbiology field. The journal has never shied away from thinking outside the box, allowing it to embrace new 
viewpoints. This will substantially benefit basic research and will help in solving the mysteries of the microbial world, whether they be antibiotic resistance, biofilm formation, QS or the more advanced microbiome studies. 


\section{Appendix 2}

Rapid Detection of Mucoid Pseudomonas aeruginosa using Microextraction of Volatiles

Wen Fan*, Gorakh Tatke*, Mimy Young, Kalai Mathee and José Almirall

* Equal Contribution (Being Submitted) 


\begin{abstract}
Pseudomonas aeruginosa is a ubiquitous Gram-negative bacterium with remarkable abilities to adapt to environmental challenges. A primary pathogen responsible for all Gram negative infections in clinical settings, it is associated with lung decline and the untimely demise of people with cystic fibrosis (CF). One of the hallmarks of $P$. aeruginosa chronic infection in CF patients is the presence of mucoid isolates that spells poor prognosis. Here we report a rapid detection technique for $P$. aeruginosa based on capillary microextraction of volatiles (CMV) using the newly invented dynamic headspace sampling device. The method is composed of a one min dynamic sampling followed by $17 \mathrm{~min}$ GC-MS analysis time. The simple, sensitive and rapid method identified 2-aminoacetophenoe and 1-undecene as biomarkers for the presence of $P$. aeruginosa. The comparison results between the mucoid and non-mucoid strains show potential use of 1undecene for differentiation of acute and chronic infection of $P$. aeruginosa using the non-invasive CMV-based breath analyzer.
\end{abstract}




\section{Introduction}

Pseudomonas aeruginosa is a Gram negative opportunistic pathogen implicated in causing systemic infections in immunocompromised and immunosuppressed individuals (Bodey et al., 1983, Ramos, 2004). Its metabolic versatility and adaptability has significantly contributed to its ubiquity (Ramos, 2004). It has increasingly been recognized as a major cause of morbidity and mortality in many clinical settings accounting for 1 in every 10 hospital-acquired infections (Abraham \& Chain) (Giamarellou, 2000). From 1975 to 2003, the percent of HAI pneumonias caused by $P$. aeruginosa nearly doubled in the US, from 9.6 to 18.1\% (Gaynes \& Edwards, 2005, Talbot et al., 2006). Unfortunately, the prognosis for these patients is often poor. Beyond high infection rate, $P$. aeruginosa also contributes to the highest case fatality rate of all Gram-negative infections (Aliaga et al., 2002). It is the primary pathogen responsible for lung decline and mortality in patients with cystic fibrosis (CF) (Doggett, 1969, Lyczak et al., 2002). In the CF lungs, transition from acute to chronic infections is marked by the development of resistance to antibiotics (Workentine et al., 2013), and conversion to a mucoid phenotype (Penketh et al., 1983, Govan \& Nelson, 1992). The mucoid phenotype is due to constitutive production of alginate (Alg), a component of extracellular polymeric (EPS) matrix (Flemming et al., 2007). Mucoidy protects the bacteria from opsonization, phagocytosis, reactive oxygen species and antibiotics (Mathee et al., 1999, Pritt et al., 2007). Thus, early detection of $P$. aeruginosa infections plays a major role in the treatment stratagem and clinical outcome. 
For early and precise detection of $P$. aeruginosa, numerous culture, biochemical, $\underline{\text { Polymerase }} \underline{\text { Chain }}$ Reaction (PCR) and serology based approaches have been developed (Qin et al., 2003, Xu et al., 2004, Tramper-Stranders et al., 2005, Tramper-Stranders et al., 2006, Daines et al., 2014). In particular, during the last decade, there has been a substantial upsurge in PCR-based approaches for early detection of $P$. aeruginosa in the respiratory samples of $\mathrm{CF}$ patients (Deschaght et al., 2011). However, in spite of the fact that PCR amplifications have been successfully used as a routine technique for identification of viruses in clinical samples (Niesters, 2002), their use in bacterial diagnostics including $P$. aeruginosa, is still limited. This is mostly due to the questionable specificity and sensitivity of these techniques. To date, $P$. aeruginosa detection and identification greatly relies on culture-based approaches which are time consuming, influenced by testing conditions and subject to physician interpretation which may lead to misinterpretation and misidentification (Deschaght et al., 2011). This together strongly suggests the need for development of new diagnostic tools that will help pave the way for a more efficient and specific $P$. aeruginosa detection techniques.

Breath analysis is a potential revolution in disease diagnostics since the collection of exhaled breath is a safe, non-invasive, easy and simple procedure, and each individual contains information regarding their own internal state (Cepelak \& Dodig, 2007, Hunter \& Dweik, 2008). Urea breath test (UBT) has been effectively used to detect Helicobacter pylori infections with a success rate of $90 \%$ (Gisbert \& Pajares, 2004). Breath analyses have also been widely tested in experimental studies to identify biomarkers that can be correlated to various 
diseases (Dent et al., 2013, Koutsokera et al., 2013, Sarbach et al., 2013). Bulk matrix of breath is largely composed of nitrogen, oxygen, carbon dioxide, water, and inert gases. However, the breath condensate contains trace amounts (parts per billion ( $\mathrm{ppb}$ ) to parts per trillion (ppt)) of inorganic gases, volatile organic compounds (VOCs), and other normally nonvolatile substances that can serve as biomarkers (Miekisch et al., 2004). For example, nitric oxide (NO) gas was reported with an increased concentration in patients with airway inflammation such as asthma and bronchiectasis (Kharitonov et al., 1997); and the odor of acetone in the breath was a characteristic feature of diabetic coma (Crofford et al., 1977).

$P$. aeruginosa has been reported to produce a "grape-like" odor. The compound responsible for this peculiar odor has been identified as 2aminoacetophenone (2-AA) by Mann in 1966 (Mann, 1966). Importantly, 2-AA was not detected in any other respiratory pathogens and thus was used as a biomarker for $P$. aeruginosa infection and/or colonization in the lung (Groenewold et al., 2011). Although specific for $P$. aeruginosa infections, 2-AA has also been identified in certain foods including corn, dairy, honey products and wine (Karagul-Yuceer et al., 2002, Fan et al., 2007, Schmarr et al., 2007, Scott-Thomas et al., 2010). Breath sample analysis of uninfected individuals shortly after eating these foods may give a false positive which could limit the diagnostic procedures (Scott-Thomas et al., 2010). In addition to 2-AA, spontaneously expectorated sputum containing $P$. aeruginosa show the presence of 2-nonanone, 2,4-dimethyl-1-heptene, 1heptene, isopentanol, and limonene (Savelev et al., 2011); and P. aeruginosa 
stationary growth culture releases dimethyl sulfide, 1-undecene, and 2-nonanone (Zscheppank et al., 2014, Briard et al., 2016).

In this study, a novel, fast, dynamic headspace sampling device, capillary microextraction of volatiles (CMV), was used to capture volatile compounds released by $P$. aeruginosa on standard culture plates. The analytical method consists of sampling the headspace above the culture plates for 1 min using the CMV device followed by inserting it into a thermal probe that is connected to a GC split/splitless (S/SL) injection port, followed by $\sim 17$ min chromatographic program of GC-MS analysis after thermal desorption of the absorbed analytes from CMV. The CMV tube (Figure A2.1) performs similarly to commercial sorbent tubes in terms of extraction efficiency, sensitivity, breakthrough and recovery. However, it does not require the complicated thermal desorption accessory normally associated with sorbent tubes. Due to its extremely high surface area and phase volume, small volume sampling $(\sim 1 \mathrm{~L})$ results in sub-ng absolute detection limits for the analytes of interest, making the CMV technique capable of detecting ppb concentrations of VOCs in air. In this study, we report the presence of the biomarkers 1-undecene and 2-AA among others from the $P$. aeruginosa. Moreover, we report that the presence of 1-undecene can be used for the differentiation between mucoid and nonmucoid strains of $P$. aeruginosa. Compare to previously described method, this is one of the first reporting of an inexpensive, fast and ultra-sensitive technique for the detection of $P$. aeruginosa that is accessible to a moderately equipped analytical laboratory requiring only GC-MS instrumentation (Table A2.1). 
Table A2.1. Comparison of extraction and analytical parameters between this work and previous reports of biomarker detection from pathogens.

\begin{tabular}{|c|c|c|c|c|}
\hline & $\begin{array}{l}\text { (Savelev et } \\
\text { al., 2011) }\end{array}$ & $\begin{array}{c}\text { (Zscheppank } \\
\text { et al., 2014) }\end{array}$ & This Report & $\begin{array}{c}\text { (Scott-Thomas } \\
\text { et al., 2010) }\end{array}$ \\
\hline $\begin{array}{l}\text { Extraction } \\
\text { Method }\end{array}$ & $\begin{array}{l}\text { SPME (DVB- } \\
\text { Carboxen- } \\
\text { PDMS), } \\
\text { closed } \\
\text { system }\end{array}$ & $\begin{array}{l}\text { NT, closed } \\
\text { system }\end{array}$ & $\begin{array}{l}\text { CMV, semi- } \\
\text { closed } \\
\text { system }\end{array}$ & $\begin{array}{c}\text { SPME } \\
\text { (Carbowax/PEG), } \\
\text { closed system }\end{array}$ \\
\hline Setup & $\begin{array}{c}\text { Easy - } \\
\text { Moderate }\end{array}$ & Moderate & Easy & \\
\hline $\begin{array}{l}\text { Extraction } \\
\text { Time }\end{array}$ & $50 \mathrm{~min}$ & $12 \min$ & $1 \mathrm{~min}$ & $5 \min$ \\
\hline $\begin{array}{l}\text { Extraction } \\
\text { Temperature }\end{array}$ & $37^{\circ} \mathrm{C}$ & & RT & \\
\hline $\begin{array}{l}\text { Injection } \\
\text { Port }\end{array}$ & Split/Splitless & & TDU & PTV-1079 \\
\hline Mode & $\begin{array}{l}\text { Pulsed } \\
\text { Splitless } \\
\text { Mode }\end{array}$ & & Split/splitless & Split (50:1) \\
\hline Detector & $\begin{array}{l}\text { El, } 70 \mathrm{eV}, \\
\text { full-scan }\end{array}$ & & & \\
\hline Column & $\begin{array}{l}\text { ZB-Wax, } 30 \\
\mathrm{~m} \times 0.25 \mathrm{~mm} \\
\text { x } 0.25 \mathrm{um}\end{array}$ & $\begin{array}{l}\text { Optima } 1,30 \\
\mathrm{~m} \times 0.32 \mathrm{um} \\
\quad \times 1 \text { um }\end{array}$ & $\begin{array}{l}\text { DB- } 5 \mathrm{~ms}, 8 \\
\mathrm{~m} \times 0.25 \mathrm{~mm} \\
\times 0.25 \mathrm{um}\end{array}$ & $\begin{array}{c}\text { ZB-Wax, } 30 \mathrm{~m} \mathrm{x} \\
0.25 \mathrm{~mm} \times 0.25 \\
\text { um }\end{array}$ \\
\hline Initial temp & $40^{\circ} \mathrm{C}, 5 \mathrm{~min}$ & $35^{\circ} \mathrm{C}$ & $40^{\circ} \mathrm{C}, 1 \mathrm{~min}$ & $60^{\circ} \mathrm{C}, 2 \min$ \\
\hline Ramp & $\begin{array}{l}5^{\circ} \mathrm{C} / \mathrm{min} \text { to } \\
100{ }^{\circ} \mathrm{C}\end{array}$ & $\begin{array}{l}1{ }^{\circ} \mathrm{C} / \mathrm{min} \text { to } \\
53{ }^{\circ} \mathrm{C}, 3 \mathrm{~min}\end{array}$ & $\begin{array}{c}15^{\circ} \mathrm{C} / \mathrm{min} \text { to } \\
115^{\circ} \mathrm{C}, 1 \\
\min \end{array}$ & $\begin{array}{c}10{ }^{\circ} \mathrm{C} / \min \text { to } 250 \\
{ }^{\circ} \mathrm{C}, 2 \min \end{array}$ \\
\hline Ramp & $\begin{array}{c}5{ }^{\circ} \mathrm{C} / \mathrm{min} \text { to } \\
150^{\circ} \mathrm{C}, 2 \\
\min \end{array}$ & $\begin{array}{c}15^{\circ} \mathrm{C} / \mathrm{min} \text { to } \\
120^{\circ} \mathrm{C}\end{array}$ & $\begin{array}{c}25^{\circ} \mathrm{C} / \mathrm{min} \text { to } \\
280{ }^{\circ} \mathrm{C}, 3 \\
\min \end{array}$ & \\
\hline Ramp & $\begin{array}{c}5^{\circ} \mathrm{C} / \mathrm{min} \text { to } \\
230{ }^{\circ} \mathrm{C}\end{array}$ & $\begin{array}{c}5^{\circ} \mathrm{C} / \mathrm{min} \text { to } \\
160{ }^{\circ} \mathrm{C}\end{array}$ & & \\
\hline Ramp & & $\begin{array}{c}2.5^{\circ} \mathrm{C} / \mathrm{min} \text { to } \\
180^{\circ} \mathrm{C}\end{array}$ & & \\
\hline Ramp & & $\begin{array}{l}2{ }^{\circ} \mathrm{C} / \mathrm{min} \text { to } \\
200{ }^{\circ} \mathrm{C}\end{array}$ & & \\
\hline Run Time & $44 \min$ & $51 \mathrm{~min}$ & $16.6 \mathrm{~min}$ & $21 \mathrm{~min}$ \\
\hline $\mathrm{He}$ & $1 \mathrm{~mL} / \mathrm{min}$ & $1.5 \mathrm{~mL} / \mathrm{min}$ & $1 \mathrm{~mL} / \mathrm{min}$ & $1.2 \mathrm{~mL} / \mathrm{min}$ \\
\hline
\end{tabular}




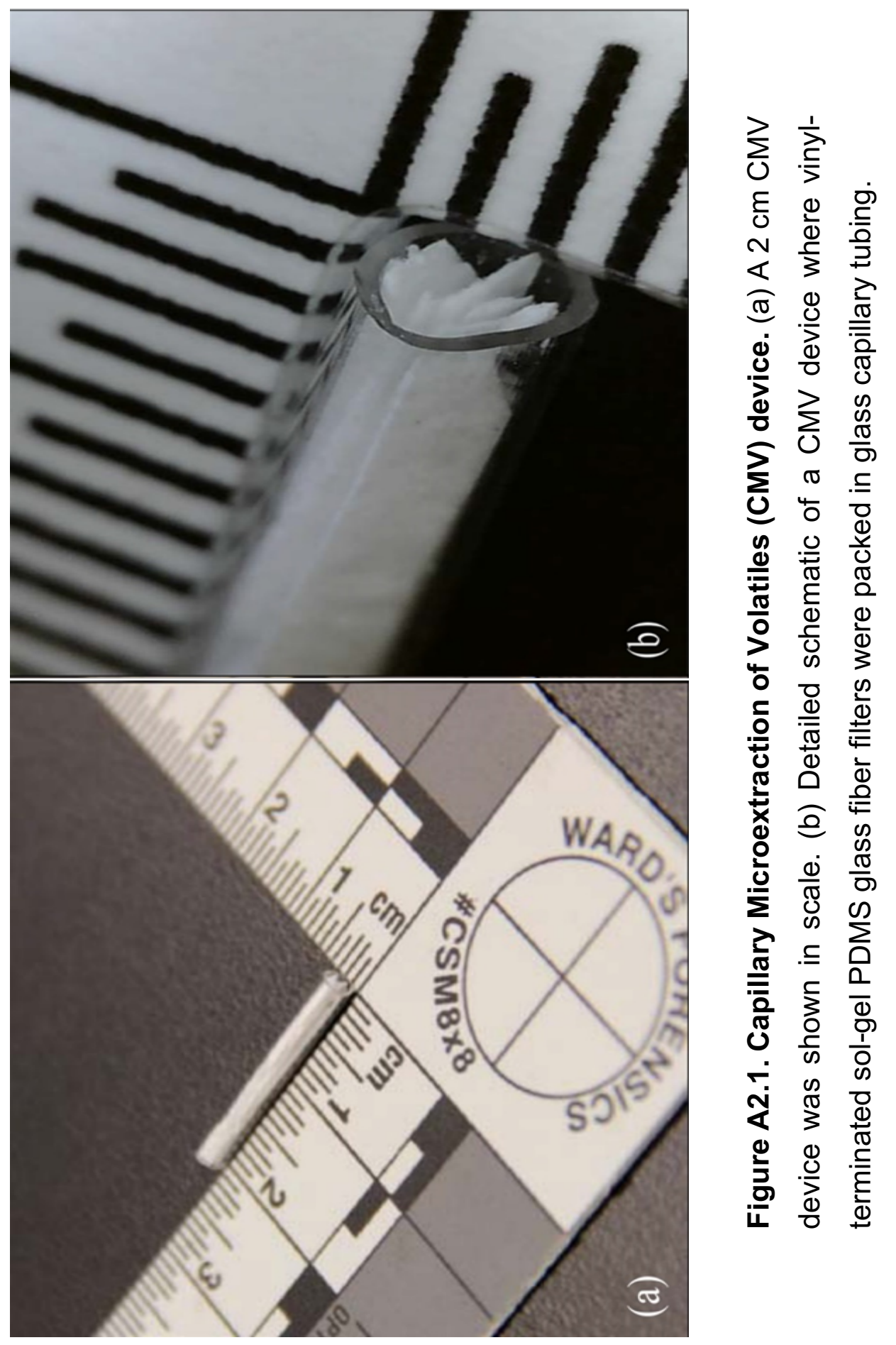




\section{Materials and Methods}

\section{Bacteria strains, media and growth conditions}

Bacterial strains used in this study are listed in Table A2.2. Luria-Bertani (LB) (Fisher Scientific) broth was used for routine cultivation of bacterial cultures and was supplemented with $1.5 \%$ agar when needed, unless specified otherwise. All cultures were grown overnight in LB broth with shaking at $200 \mathrm{rpm}$, at $37^{\circ} \mathrm{C}$ for Pseudomonas aeruginosa (Mucoid/Non-mucoid), Staphylococcus aureus, Escherichia coli, $26^{\circ} \mathrm{C}$ for Serratia marcescens, Chromobacterium violaceum and $30^{\circ} \mathrm{C}$ for Bacillus strains. Briefly, overnight cultures were diluted to obtain equal optical densities. Culture densities were normalized to a final $A_{600}$ of $0.05-0.08$ before inoculating on LB agar plates by swabbing the surface with adjusted culture suspensions. Plates were allowed to dry before incubating overnight for 12 to 16 h at $37^{\circ} \mathrm{C}, 30^{\circ} \mathrm{C}$ and $/$ or $26^{\circ} \mathrm{C}$ depending on the culture. In addition, mixed culture suspension of $P$. aeruginosa prototypic PAO1, E. coli (DH5a), S. marcescens, B. cereus and S. aureus in different combination were used. For mixed bacterial cultures, the plates were incubated overnight at $30^{\circ} \mathrm{C}$. In total, 280 culture plates

were sampled, and each strain was analyzed at least three times. All the sampling and analyses were accomplished using blind study method. The culture plates containing all the bacteria, including the negative controls, were labeled with reference IDs to minimize any bias during the headspace sampling. The LB agar plate with no culture was used as a negative control. 


\section{Dynamic headspace sampling using CMV devices}

The CMV devices were recently developed and reported as an efficient way to detect trace amounts of explosives (Fan \& Almirall, 2014). Fabrication of the CMV devices has been described elsewhere (Fan \& Almirall, 2014). These 2-cm length open-ended sampling devices allow for fast dynamic headspace sampling. The CMV devices were conditioned in an oven at $250{ }^{\circ} \mathrm{C}$ for an hour prior to use, cooled to room temperature and connected to a hand-held air monitoring vacuum pump (Escort Elf Air Sampling Pump, Zefon International Inc., Ocala, FL) that provides a flow through the CMV device at the rate of 1.5 liters per min (LPM). For the culture plates sampling, the LB agar culture plates were popped open with a small opening where the CMV devices were simply inserted into the plates for 1 min dynamic sampling at a flow rate of $1.5 \mathrm{~L} / \mathrm{min}$ (Figure A2.2). The CMV sampling device capable of sub-nanogram detection of VOCs, when coupled to GC-MS (Fan \& Almirall, 2014, Tarifa \& Almirall, 2015) (Air Chemistry, Inc, Miami, FL).

\section{Sample analysis using GC-MS}

After 1 min sampling, the CMV device was disconnected from the tubing and inserted into a probe for thermal desorption into an Agilent Technologies 7890A GC S/SL injector that was connected to thermal separation probe adaptor from Agilent Technologies Inc., Santa Clara, CA. The injector was set to $180{ }^{\circ} \mathrm{C}$. The Agilent Technologies 7890A GC system was equipped with a 5975C inert XL mass spectrometer detector (MSD). The GC column used in this research was a DB-5ms Ultra Inert, $8 \mathrm{~m} \times 0.25 \mathrm{~mm} \times 0.25 \mu \mathrm{m}$ column. For the initial study, the GC oven temperature ramp started at $40{ }^{\circ} \mathrm{C}$, was held for 1 min and ramped at 15 
${ }^{\circ} \mathrm{C} / \mathrm{min}$ to $200{ }^{\circ} \mathrm{C}$ and held for $1 \mathrm{~min}$, ramped again at $15{ }^{\circ} \mathrm{C} / \mathrm{min}$ to $240{ }^{\circ} \mathrm{C}$, held for $6.5 \mathrm{~min}$ and ramped at $25{ }^{\circ} \mathrm{C} / \mathrm{min}$ to $270{ }^{\circ} \mathrm{C}$, ramped at $5{ }^{\circ} \mathrm{C} / \mathrm{min}$ to a final temperature of $280{ }^{\circ} \mathrm{C}$ and held for $4 \mathrm{~min}$. The total analyses time was $29.33 \mathrm{~min}$ that provided with a high resolution for detection of various volatile organic compounds in the headspace over the culture plates. Once biomarkers were identified for $P$. aeruginosa strains, a fast GC oven temperature program was used to reduce the analyses time. The oven temperature started at $40^{\circ} \mathrm{C}$ for $1 \mathrm{~min}$, and ramped to $115^{\circ} \mathrm{C}$ at $15^{\circ} \mathrm{C} / \mathrm{min}$ and held for $1 \mathrm{~min}$, and ramped to final temperature of $280^{\circ} \mathrm{C}$ at $25^{\circ} \mathrm{C} / \mathrm{min}$ and held for $3 \mathrm{~min}$. The total analyses time was reduced to $16.6 \mathrm{~min}$. 


\section{Results}

\section{Identification of VOCs in the Headspace of $P$. aeruginosa}

The VOCs present in the prototypic $P$. aeruginosa strain PAO1 were differentiated from other bacteria, E. coli $\mathrm{DH} 5 \alpha$ and C. violaceum, in this particular study and characteristic VOCs were found in the $\mathrm{P}$ aeruginosa strains using the 1-min. headspace sampling method. After 24 hours of incubation, the headspace of the plates were sampled with the CMV for $1 \mathrm{~min}$. 1-Undecene and 2aminoacetophenone (2-AA) were detected in $P$. aeruginosa, and indoles were detected in E. coli (Figure A2.3). After 48 hours of incubation at room temperature, limonene was also detected in both the $P$. aeruginosa and $C$. violaceum plates; thus, limonene wasn't chosen as a biomarker along with 1-undecene and 2-AA in this research.

\section{Differentiation of $\boldsymbol{P}$. aeruginosa from Other Bacterial Species}

The 1-min. headspace sampling using CMV resulted in the differentiation of the 199 plates containing $P$. aeruginosa strains from the 66 plates containing other bacteria using blind sampling studies. The compounds 1-undecene and 2-AA were found only in the headspace of the $P$. aeruginosa strains (see Table A2.3) and these results are consistent with previous workers who also reported finding these compounds as biomarkers of $P$. aeruginosa. Almost every plate containing the $P$. aeruginosa strain resulted in the detection of one or both of these biomarkers. Additionally, none of the plates containing the other bacteria resulted in the

presence of either or both of the biomarkers. A confusion matrix was built (Table A2.3) in which the theoretical biomarker present is equivalent to $P$. aeruginosa 
plate and the theoretical biomarker absence is equivalent to a plate containing other bacteria. Based on this confusion matrix, two observations can be made. First, where the true negative rate equals to 1.00 indicates that the two biomarkers chosen are not found in the other bacterial strains tested in this study. Second, the false negative rate of 0.12 indicates that a few $P$. aeruginosa strains do not emit either 1-undecene or 2-AA. Mucoid strains may be retaining the VOCs better than the non-mucoid strains and therefore the biomarkers are more difficult to detect.

\section{Detection of 2-AA and 1-undecene is specific for $P$. aeruginosa}

To determine if this detection method is specific and sensitive, several mixtures of species were made (Table A2.4). The headspace of each mixture was also sampled and the chromatograms were overlaid (Figure A2.4). As predicted, the two compounds, 1-undecene and 2-AA were detected in Group 1 ( $P$. aeruginosa, S. marcescens, and $S$. aureus) and 4 ( $P$. aeruginosa alone), and indole was found in Group 2 (E. coli, S. marcescens, and B. cereus UW85), 3 (E. coli, S. marcescens, and S. aureus) and 5 (E.coli alone). Thus, the test method for 1 min fast dynamic sampling followed by analysis in GC-MS is suitable even when a complex mixture is present.

\section{Differentiation between mucoid and non-mucoid $P$. aeruginosa strains}

$P$. aeruginosa strains often transition from acute to chronic infections in patients with CF. The transition is marked by development of resistance to antibiotics and conversion to mucoid phenotype. The mucoid phenotype is due to overproduction of alginate (Figure A2.5). The mucoid strains are referred to as $\mathrm{Alg}^{+}$. Thus we hypothesized that the transition will lead to concomitant change in 
odor the profile. To test this hypothesis, eight non-mucoid and 10 mucoid strains were tested (Figure A2.6).

For the eight mucoid strains analyzed, 1-undecene was detected in all 83 plates (100\% detection rate); while $2-\mathrm{AA}$ was found in 80 plates $(96.4 \%$ detection rate) (Figure A2.6). The only strain that didn't have 2-AA in headspace is strain CDN118, which is a pan-resistant bacterimic isolate from Nigeria. The absence of 2-AA in this single strain needs further study.

The 2-AA biomarker was detected in 85 of $116(73.3 \%)$ of the mucoid strains (Figure 2A.6). The plates that failed to have detectable 2-AA in the headspace included strain ZW77 (3 plates), PAO1 (3 plates), and PDO300 (17 plates). The 1undecene was detected in all of the non-mucoid (Alg-) strains whereas only 41.3 $\%$ of the mucoid isolates presented 1-undecene in the headspace (Figure 2A.6). The mucoid strains may not produce appreciable amounts of 1-undecene. The presence of 1-undecene in some of the plates could result from the presence of non-mucoid strains in the plates due to their ability to transition during the incubation process. In fact, the $\mathrm{Alg}^{+}$phenotype of PDO300 is unstable and up to $80 \%$ of the isolates can revert to non-mucoid phenotype (Sautter et al., 2012). To test this hypothesis, volatiles from three stable mucoid strains PA2192 (12 plates), FRD39 (13 plates), were compared with the Alg- PAO1 (6 plates) strains. As expected, only 2-AA was found in all 31 plates. The stable mucoid strains were differentiated from the non-mucoid strains by only the presence of these two biomarkers using the CMV headspace test described. 


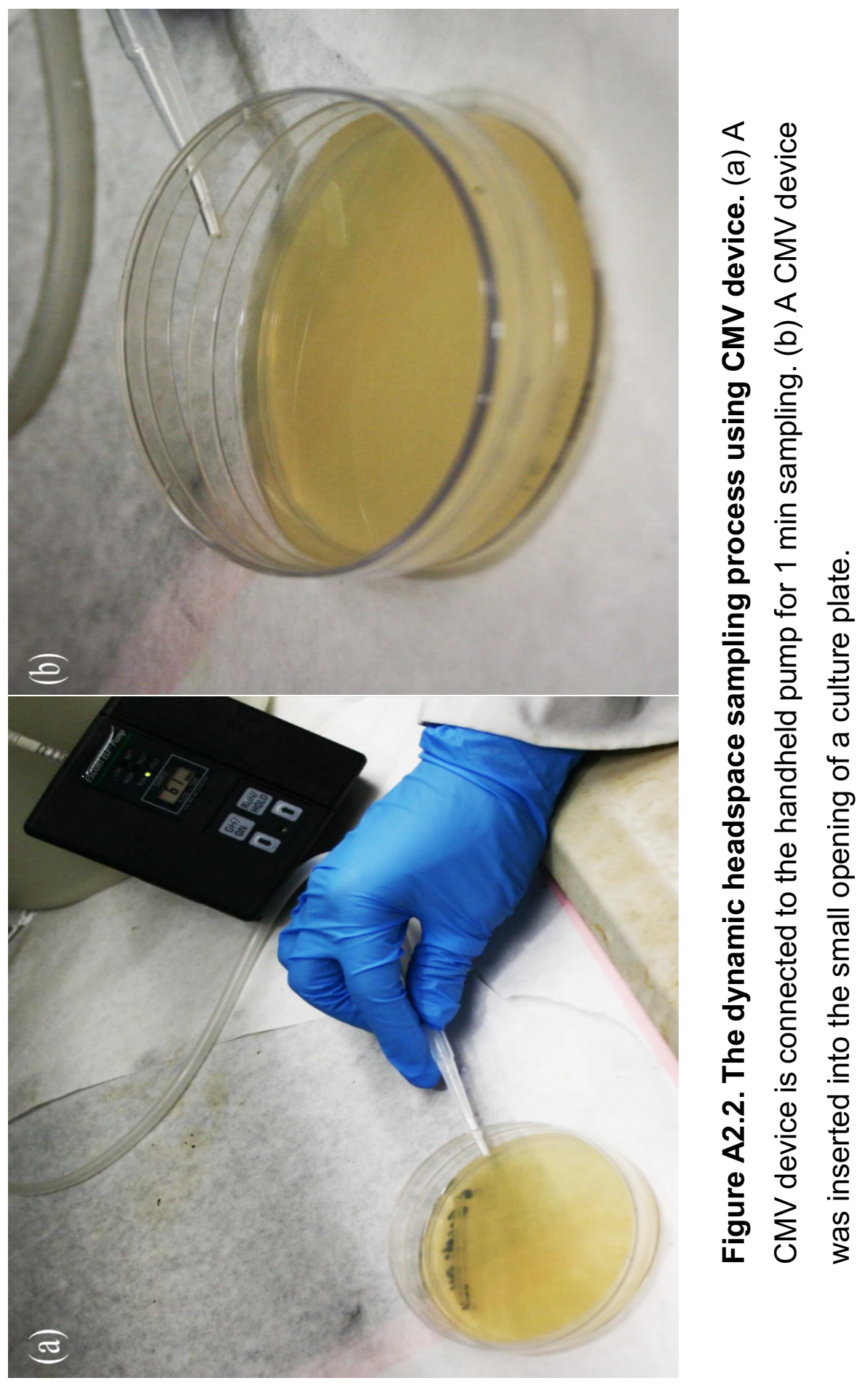




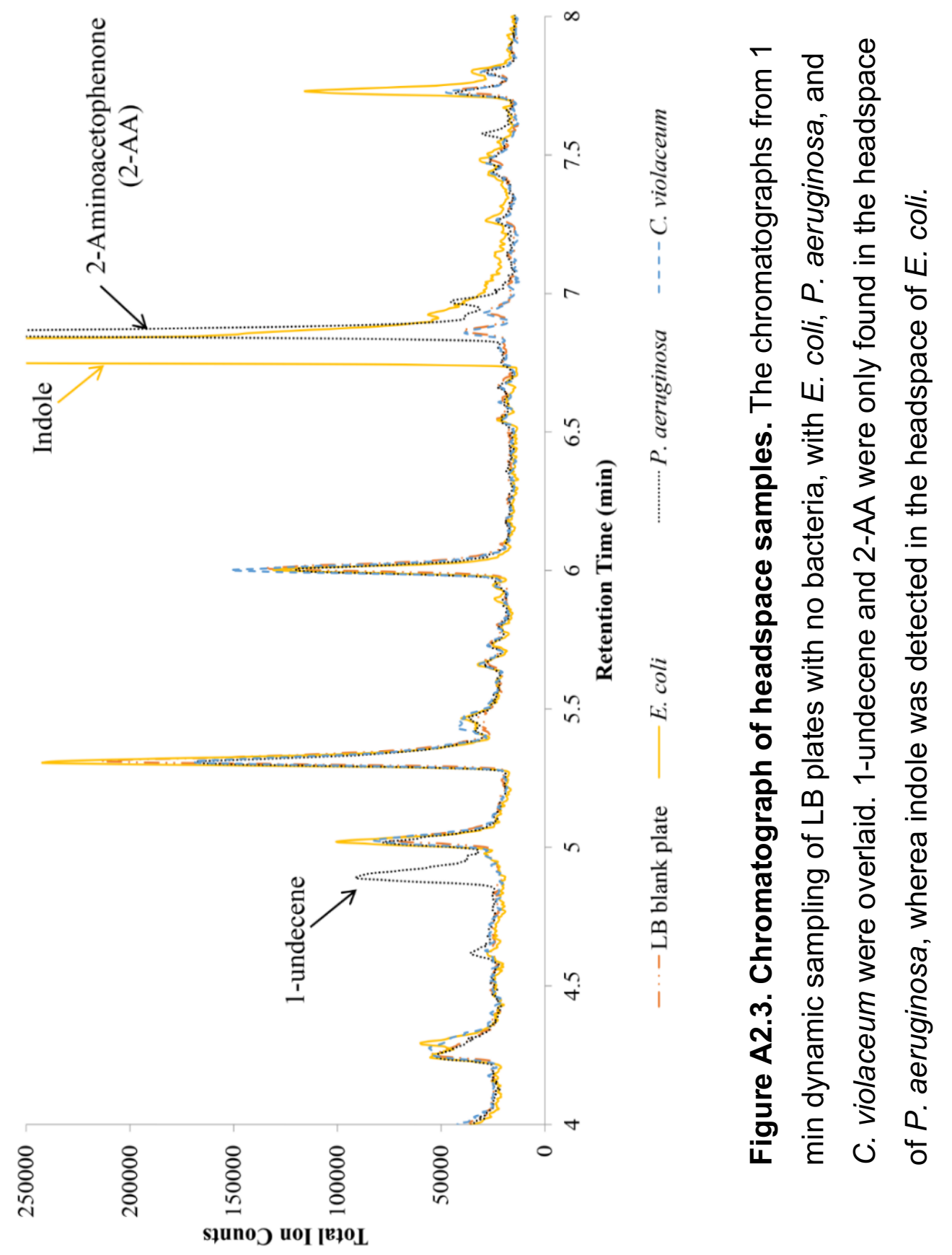




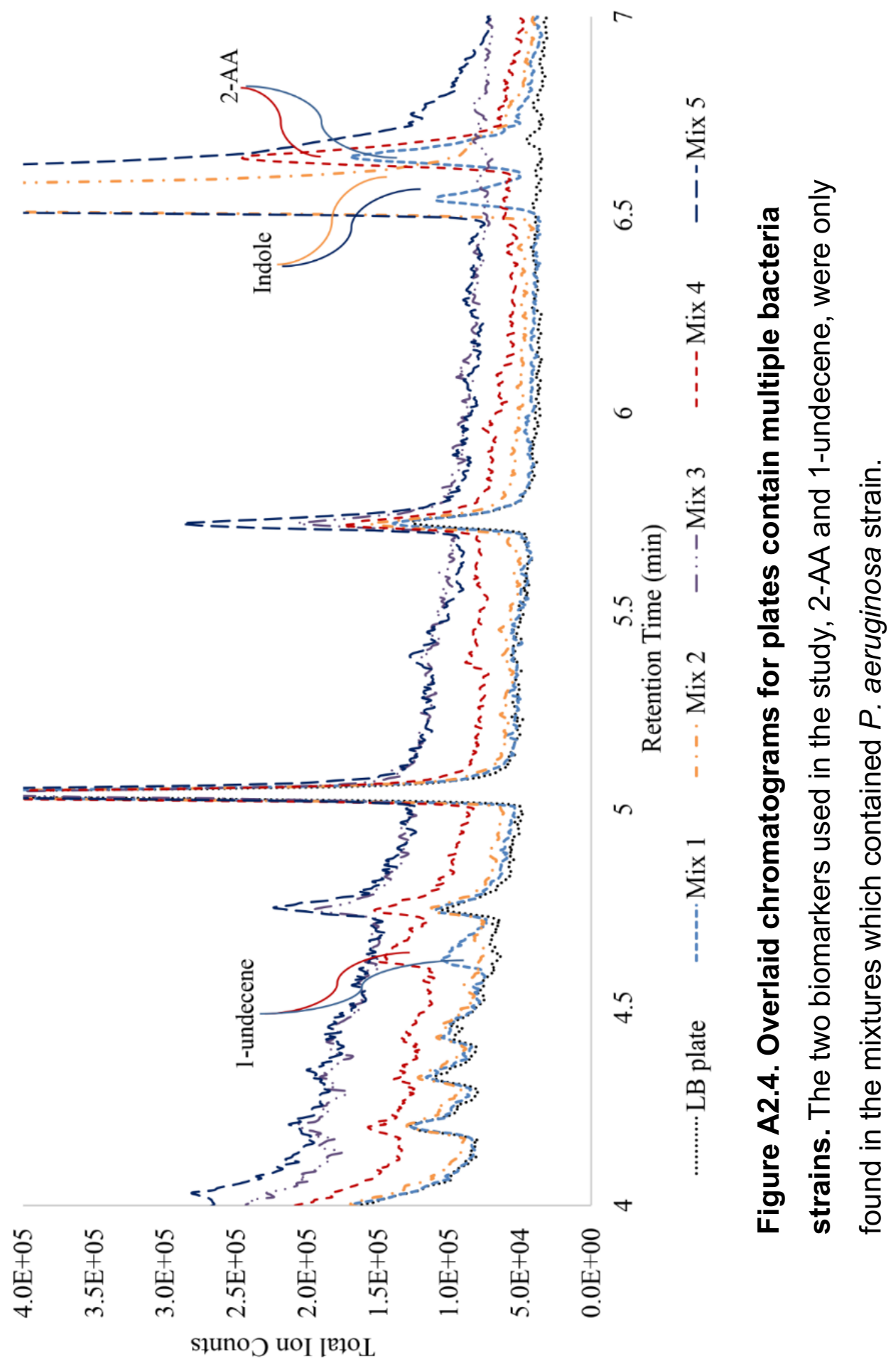



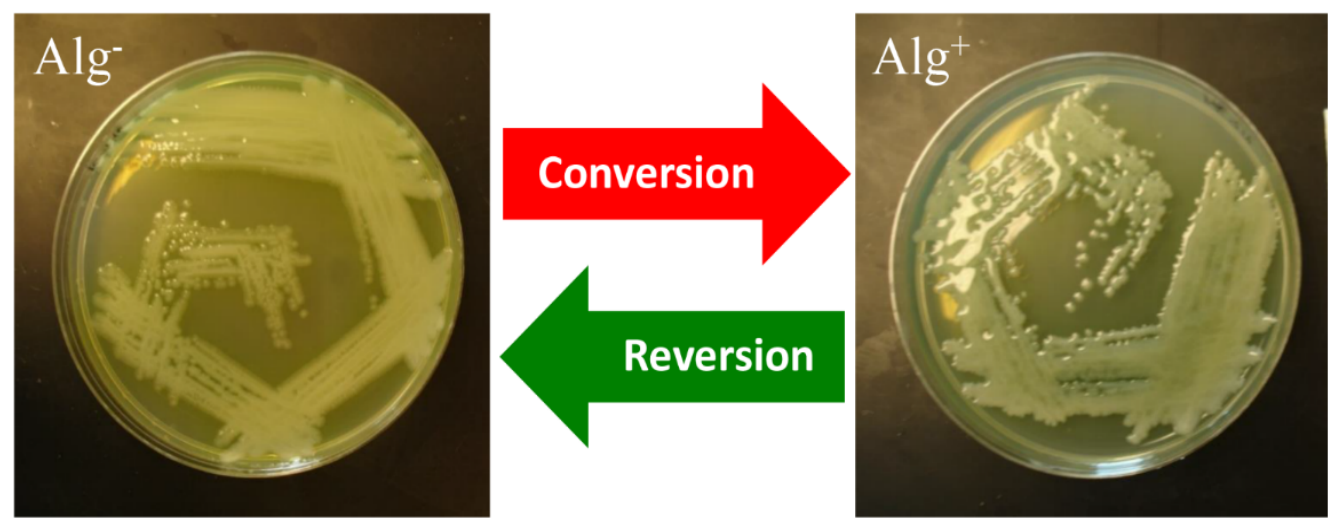

Figure A2.5. Non-mucoid (Alg) and mucoid ( $\left.\mathrm{Alg}^{+}\right)$P. aeruginosa. The prototypic $P$. aeruginosa such as PAO1 are non-mucoid. The conversion seen during chronic infection, especially in patients with cystic fibrosis is due to a mutation in mucA encoding an anti-sigma factor. The mucoid phenotype reverts back to non-mucoidy in the absence of selection due to second site suppressors strain (Sautter et al., 2012).

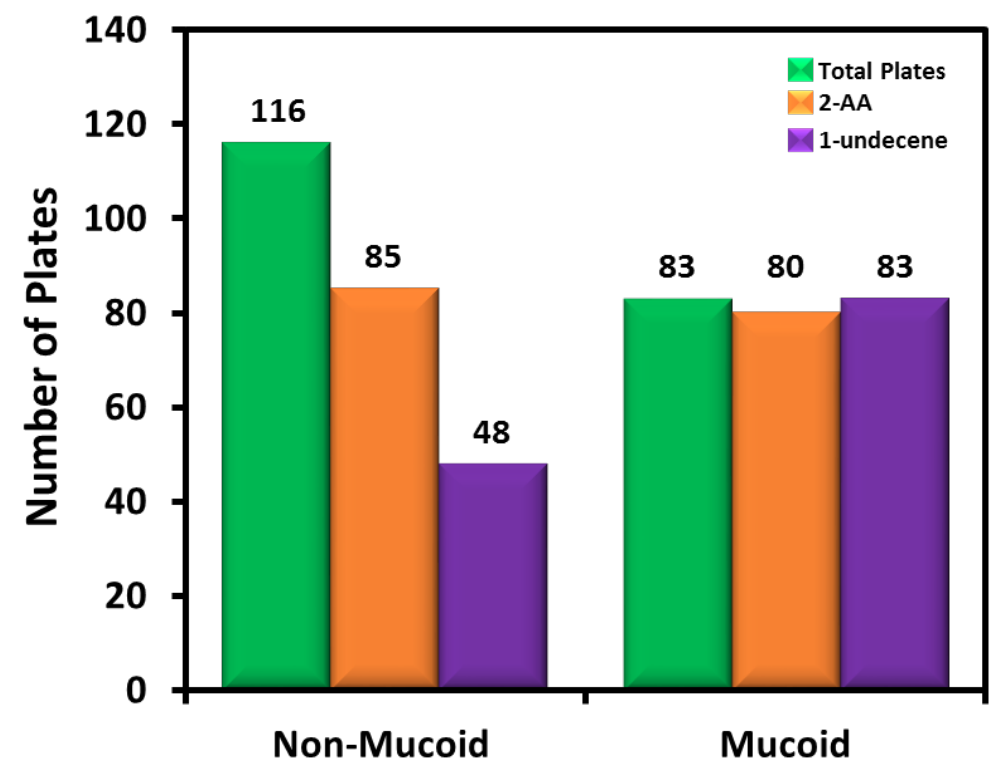

Figure A2.6. The detection frequency of 1-undecene and 2-aminoacetophenone (2-AA) in mucoid and non-mucoid $\boldsymbol{P}$. aeruginosa strains. 1-Undecene was $100 \%$ detectable in non-mucoid $P$. aeruginosa strains, while only $43 \%$ detectable in mucoid strains. The significant drop in detection frequency led to the hypothesis of using 1undecene as a biomarker for separation of mucoid and non-mucoid strains. 
Table A2.2. Bacterial strains used in this study

\begin{tabular}{|c|c|c|c|}
\hline Strain ID & $\begin{array}{c}\text { Strain } \\
\text { Background }\end{array}$ & Relevant characteristics & Source \\
\hline \multicolumn{4}{|c|}{ P. aeruginosa strains } \\
\hline PAO1 & PAO1 & $\begin{array}{l}\text { Alg; Prototypic wild type, a } \\
\text { wound isolate }\end{array}$ & $\begin{array}{l}\text { (Stover et al., } \\
\text { 2000) }\end{array}$ \\
\hline PDO300 & PAO1 & $\begin{array}{l}\mathrm{Alg}^{+} ; \mathrm{PAOmucA22} \\
\text { constitutively mucoid }\end{array}$ & $\begin{array}{l}\text { (Mathee et al., } \\
\text { 1999) }\end{array}$ \\
\hline PKM315 & PAO1 & Alg; PAO $\triangle a m p R$ & $\begin{array}{l}\text { (Balasubramania } \\
\mathrm{n} \text { et al., 2012) }\end{array}$ \\
\hline PKM816 & PDO300 & $\begin{array}{l}\text { Alg; PDO300 revertant, non- } \\
\text { mucoid; } \\
\text { PAOlagO96 mucA22 }\end{array}$ & $\begin{array}{l}\text { sap26; Mathee } \\
\text { Lab }\end{array}$ \\
\hline PKM831 & PDO300 & $\begin{array}{l}\text { Alg; PDO300 revertant, non- } \\
\text { mucoid; PAOmucP451 mucA22 }\end{array}$ & $\begin{array}{l}\text { sap21; Mathee } \\
\text { Lab }\end{array}$ \\
\hline PKM900 & PAO1 & $\begin{array}{l}\text { Alg; PAO } \triangle \text { mifR; in-frame } \\
\text { deletion of mifS }\end{array}$ & $\begin{array}{l}\text { (Tatke et al., } \\
\text { 2015) }\end{array}$ \\
\hline PKM901 & PAO1 & $\begin{array}{l}\text { Alg; PAO } \triangle \text { mifS; In-frame } \\
\text { deletion of mifS }\end{array}$ & $\begin{array}{l}\text { (Tatke et al., } \\
\text { 2015) }\end{array}$ \\
\hline PKM902 & PAO1 & $\begin{array}{l}\text { Alg; PAO } \triangle \text { mifSR; in-frame } \\
\text { deletion of } \text { mifS and mifR }\end{array}$ & $\begin{array}{c}\text { (Tatke et al., } \\
\text { 2015) }\end{array}$ \\
\hline CDN118 & & $\begin{array}{l}\text { Alg; Panresistant clinical isolate } \\
\text { from Nigeria }\end{array}$ & C.D. Nkwonta \\
\hline PA2192 & 2192 & $\begin{array}{l}\mathrm{Alg}^{+} \text {; Mucoid cystic fibrosis (CF) } \\
\text { clinical isolate }\end{array}$ & (Pier et al., 1983) \\
\hline FRD1 & FRD1 & $\mathrm{Alg}^{+}$; Mucoid CF clinical isolate & $\begin{array}{c}\text { (Ohman \& } \\
\text { Chakrabarty, } \\
\text { 1981) }\end{array}$ \\
\hline FRD39 & FRD1 & $\mathrm{Alg}^{+}$; Mucoid isolate & $\begin{array}{l}\text { (Ohman \& } \\
\text { Chakrabarty, } \\
\text { 1981) }\end{array}$ \\
\hline ZW77 & & $\begin{array}{l}\text { Alg+; poxB15, CF throat swab, } \\
\text { London, UK, } 1997\end{array}$ & $\begin{array}{l}\text { (Kong et al., } \\
\text { 2005) }\end{array}$ \\
\hline ZW98 & & $\begin{array}{l}\text { Alg; CF throat swab, The } \\
\text { Hague, Netherlands, } 1997\end{array}$ & $\begin{array}{l}\text { (Kong et al., } \\
\text { 2005) }\end{array}$ \\
\hline PT22 & & $\begin{array}{l}\text { Alg”; Water, Mühlheim, } \\
\text { Germany, } 1992\end{array}$ & $\begin{array}{l}\text { (Kong et al., } \\
\text { 2005) }\end{array}$ \\
\hline $641 \mathrm{HD}$ & & $\begin{array}{l}\text { Alg; poxB22; Water, Mühlheim, } \\
\text { Germany, } 1992\end{array}$ & $\begin{array}{l}\text { (Kong et al., } \\
2005)\end{array}$ \\
\hline PKM122 & PAO1 & $\begin{array}{l}\mathrm{Alg}^{+} ; \text {PAOmucA22; mucoid } \\
\text { strain isolated from PAO1 } \\
\text { biofilm after PMN exposure }\end{array}$ & $\begin{array}{l}\text { (Mathee et al., } \\
\text { 1999) }\end{array}$ \\
\hline
\end{tabular}




\begin{tabular}{|c|c|c|c|}
\hline PKM123 & PAO1 & $\begin{array}{l}\text { Alg }^{+} \text {; PAOmucA22; mucoid } \\
\text { strain isolated from PAO1 } \\
\text { oiofilm after PMN exposure }\end{array}$ & $\begin{array}{c}\text { (Mathee et al., } \\
\text { 1999) }\end{array}$ \\
\hline \multicolumn{4}{|c|}{ Bacillus strains } \\
\hline $\begin{array}{l}\text { ATCC } \\
35866\end{array}$ & $\begin{array}{c}B . \\
\text { thuringiensis }\end{array}$ & NRRL B4488 [HD73] & ATCC \\
\hline $\begin{array}{l}\text { ATCC } \\
23857\end{array}$ & B. subtilis & ind- tyr+ & ATCC \\
\hline $\begin{array}{l}\text { ATCC } \\
14581\end{array}$ & B. megaterium & & ATCC \\
\hline $\begin{array}{l}\text { ATCC } \\
53522 \\
\end{array}$ & $\begin{array}{l}\text { B. cereus } \\
\text { UW85 }\end{array}$ & & ATCC \\
\hline \multicolumn{4}{|c|}{ Other bacterial strains } \\
\hline CV026 & $\begin{array}{c}\text { Chromobacteriu } \\
m \text { violaceum }\end{array}$ & Violaceum negative mutant & (Pier et al., 1983) \\
\hline $\mathrm{DH} 5 \alpha$ & Escherichia coli & $\begin{array}{l}\mathrm{F}^{-} \text {Ф80lacZ MM15 } \Delta \text { (lacZYA- } \\
\text { argF)U169 deoR recA1 } \\
\left.\text { endA1 hsdR17 (rk } \mathrm{mk}^{+}\right) \\
\text {phoA supE44 } \lambda^{-} \text {thi-1 gyrA96 } \\
\text { relA1 }\end{array}$ & $\begin{array}{c}\text { New England } \\
\text { Biolabs }\end{array}$ \\
\hline $\begin{array}{l}\text { ATCC } \\
13880\end{array}$ & $\begin{array}{c}\text { Serratia } \\
\text { marcescens }\end{array}$ & Isolated from pond water & ATCC \\
\hline KM1000 & $\begin{array}{c}\text { Staphylococcus } \\
\text { aureus }\end{array}$ & Wild type & Mathee Lab \\
\hline
\end{tabular}

Table A2.3. Confusion matrix built upon the blind study.

\section{Theoretical}

Confusion Matrix

\section{Biomarker}

Present

Biomarker Present

\section{Experimental}

\section{Biomarker not}

Present
Biomarker not Present

$0(\mathrm{FP}=0)$

$$
23(\mathrm{FN}=0.12) \quad 66(\mathrm{TN}=1.00)
$$

TP: True Positive Rate; FP: False Positive Rate; FN: False Negative Rate; TN: True Negative Rate 
Table A2.4. Mixture of bacterial cultures

\begin{tabular}{lcc}
\hline & Strain Background & Strain IDs \\
\hline P. aeruginosa & PAO1 \\
& S. marcescens & ATCC 13880 \\
& S. aureus & KM1000 \\
\hline Mixture 2 & E. coli & DH5a \\
& S. marcescens & ATCC 13880 \\
& B. cereus UW85 & ATCC 53522 \\
\hline Mixture 3 & E. coli & DH5a \\
\hline S. marcescens & ATCC 13880 \\
\hline Sixture 4 & P. aureus & KM1000 \\
\hline
\end{tabular}




\section{Discussion}

The rapid sampling technique using CMV devices coupled to GC-MS for sample analysis is an effective method to differentiate $P$. aeruginosa strains from the other bacterial pathogens. The two biomarkers used in this study, 2-AA and 1undecene, are only found in the headspace of $P$. aeruginosa strains, in comparison to the headspace of several other pathogen strains. No significant levels of biomarkers were found for the other bacterial pathogens except that indole was found in the headspace of $\mathrm{DH} 5 \alpha(E$. coli). Beyond this, when multiple bacteria were mixed together, the method doesn't show any interference and is specific in identifying the two biomarkers only from the mixtures that contained the $P$. aeruginosa strains.

In addition, to differentiate $P$. aeruginosa strains from the other bacterial pathogens, the method could also be used for separation of $P$. aeruginosa mucoid and non-mucoid strains. In the analysis of 8 mucoid strains, the 1-undecene detection rate dropped significantly from $100 \%$ to $41.3 \%$, when compared to the analysis of 10 non-mucoid strains. These results suggest that either the mucoid strains do not produce 1-undecene in the headspace as a biomarker or the mucoidy prevents the release (absorbs) the 1-undecene. The headspace analysis of the three stable mucoid strains shows that 2-AA was the only biomarker detected in the headspace. Previously, solid phase microextraction (SPME) has been used for studying the headspace of $P$. aeruginosa (Scott-Thomas et al., 2010, Savelev et al., 2011). Because of the static sampling nature of the SPME device and its limited surface area and phase volume, the static sampling SPME 
methods required longer analysis times. In addition, the SPME glass fiber is very fragile and thus not suitable for field applications. In this study, the newly developed method is rugged, dynamic, fast, and has comparable or better sensitivity as SPME. However, in the analysis of about 300 culture plates, a relatively low true positive rate ( $88 \%$ ) was found which resulted from three out of eight mucoid strains. As discussed above, the mucoid strains may have the polysaccharide cover to prevent the release of biomarkers into the headspace; thus, culturing the plates under a condition to prevent conversion from mucoid to non-mucoid might be useful for successful detection that can lead to a higher true positive rate. Additionally, both stable and unstable mucoid strains should be analyzed. Further experiments should also include quantitative analysis that addresses the number of bacteria on the culture plate versus the headspace biomarker signals. These studies may provide further insights into the concentration of biomarker compounds that can differentiate the mucoid and nonmucoid strains of $P$. aeruginosa. 


\section{Conclusions}

The newly reported CMV device for dynamic headspace sampling of VOCs over bacteria culture plates provides for a simple and inexpensive extraction setup. The 1 min sampling time significantly increases the sampling throughput and the direct introduction of the CMV into a port of a GC-MS followed by thermal desorption produces a VOC profile in $\sim 17$ min for each plate. 1-undecene and 2AA were identified as the biomarkers for $P$. aeruginosa in these studies. In further screening test, 265 plates were sampled and no false positive was generated with a total number of 66 other bacteria (not containing $P$. aeruginosa) plates analyzed. In the analysis of non-mucoid strains, the clinical isolate CDN118 did not produce 2-AA in the headspace, but 1-undecene was found in all plates; thus, 1-undecene can be used as the primary biomarker while 2-AA as a secondary marker for confirmation. In addition, 1-undecene might be used as the biomarker to differentiate mucoid and non-mucoid $P$. aeruginosa isolates. The method was also successfully applied to the culture plates with multiple bacteria (mixtures). The detection results showed the method is sensitive and effective even with a relative complex matrix. In conclusion, the simple and fast sampling method developed in this research is effective in screening bacteria culture plates to identify $P$. aeruginosa strains. Additional developmental research may lead to rapid, noninvasive detection of $P$. aeruginosa in the breath of patients that are susceptible to $P$. aeruginosa infection such as potential cystic fibrosis patients. 


\section{BIBLIOGRAPHY}

Methods for dilution antimicrobial susceptibility tests for bacteria that grow aerobically; Approved standards (2012). Wayne (Pennsylvania). Clinical and Laboratory Standards Institute 32: M07-A09.

Abraham EP \& Chain E (1988) An enzyme from bacteria able to destroy penicillin. 1940. Rev Infect Dis 10: 677-678.

Adam B, Baillie GS \& Douglas LJ (2002) Mixed species biofilms of Candida albicans and Staphylococcus epidermidis. J Med Microbiol 51: 344-349.

Adonizio A, Leal SM, Jr., Ausubel FM \& Mathee K (2008) Attenuation of Pseudomonas aeruginosa virulence by medicinal plants in a Caenorhabditis elegans model system. J Med Microbiol 57: 809-813.

Adonizio AL, Downum K, Bennett BC \& Mathee K (2006) Anti-quorum sensing activity of medicinal plants in southern Florida. J Ethnopharmacol 105: 427-435.

Al-Fattani MA \& Douglas LJ (2006) Biofilm matrix of Candida albicans and Candida tropicalis: chemical composition and role in drug resistance. J Med Microbiol 55: 999-1008.

Albright LM, Huala E \& Ausubel FM (1989) Prokaryotic signal transduction mediated by sensor and regulator protein pairs. Annual review of genetics 23 : 311-336.

Aliaga L, Mediavilla JD \& Cobo F (2002) A clinical index predicting mortality with Pseudomonas aeruginosa bacteraemia. J Med Microbiol 51: 615-619.

Allen L, Dockrell DH, Pattery T, Lee DG, Cornelis P, Hellewell PG \& Whyte MK (2005) Pyocyanin production by Pseudomonas aeruginosa induces neutrophil apoptosis and impairs neutrophil-mediated host defenses in vivo. J Immunol 174: 3643-3649.

Alm E, Huang K \& Arkin A (2006) The evolution of two-component systems in bacteria reveals different strategies for niche adaptation. PLoS Comput Biol 2: e143. 
Amyes SG (1989) The success of plasmid-encoded resistance genes in clinical bacteria. An examination of plasmid-mediated ampicillin and trimethoprim resistance genes and their resistance mechanisms. J Med Microbiol 28: 73-83.

Anderson JD, Gillespie WA \& Richmond MH (1973) Chemotherapy and antibiotic-resistance transfer between Enterobacteria in the human gastrointestinal tract. J Med Microbiol 6: 461-473.

Arora SK, Ritchings BW, Almira EC, Lory S \& Ramphal R (1997) A transcriptional activator, FleQ, regulates mucin adhesion and flagellar gene expression in Pseudomonas aeruginosa in a cascade manner. J Bacteriol 179: 5574-5581.

Asai K, Baik SH, Kasahara Y, Moriya S \& Ogasawara N (2000) Regulation of the transport system for C4-dicarboxylic acids in Bacillus subtilis. Microbiol 146 263271.

Bains M, Fernandez L \& Hancock RE (2012) Phosphate starvation promotes swarming motility and cytotoxicity of $P$ seudomonas aeruginosa. Applied and environmental microbiology 78: 6762-6768.

Balasubramanian D, Schneper L, Kumari H \& Mathee K (2013) A dynamic and intricate regulatory network determines $P$ seudomonas aeruginosa virulence. Nucleic Acids Res 41: 1-20.

Balasubramanian D, Schneper L, Merighi M, Smith R, Narasimhan G, Lory S \& Mathee K (2012) The regulatory repertoire of Pseudomonas aeruginosa AmpC $\beta$ lactamase regulator AmpR includes virulence genes. PLoS One 7: e34067.

Balasubramanian D, Schneper L, Merighi M, Smith R, Narasimhan G, Lory S \& Mathee K (2012) The regulatory repertoire of Pseudomonas aeruginosa AmpC ss-lactamase regulator AmpR includes virulence genes. PloS one 7: e34067.

Banin E, Vasil ML \& Greenberg EP (2005) Iron and Pseudomonas aeruginosa biofilm formation. Proc Natl Acad Sci U S A 102: 11076-11081.

Barakat M, Ortet P, Jourlin-Castelli C, Ansaldi M, Mejean V \& Whitworth DE (2009) P2CS: a two-component system resource for prokaryotic signal transduction research. BMC Genomics 10: 315. 
Baron SS \& Rowe JJ (1981) Antibiotic action of pyocyanin. Antimicrobial agents and chemotherapy 20: 814-820.

Barraud N, Hassett DJ, Hwang SH, Rice SA, Kjelleberg S \& Webb JS (2006) Involvement of nitric oxide in biofilm dispersal of Pseudomonas aeruginosa. $J$ Bacteriol 188: 7344-7353.

Barrios H, Valderrama B \& Morett E (1999) Compilation and analysis of sigma(54)-dependent promoter sequences. Nucleic Acids Res 27: 4305-4313.

Bascom-Slack CA \& Dawson D (1998) A physical assay for detection of early meiotic recombination intermediates in Saccharomyces cerevisiae. Mol Gen Genet 258: 512-520.

Batista S, Patriarca EJ, Tate R, Martinez-Drets G \& Gill PR (2009) An alternative succinate (2-oxoglutarate) transport system in Rhizobium tropici is induced in nodules of Phaseolus vulgaris. J Bacteriol 191: 5057-5067.

Beare PA, For RJ, Martin LW \& Lamont IL (2003) Siderophore-mediated cell signalling in Pseudomonas aeruginosa: divergent pathways regulate virulence factor production and siderophore receptor synthesis. Mol Microbiol 47: 195-207.

Behrends V, Bell TJ, Liebeke M, Cordes-Blauert A, Ashraf SN, Nair C, Zlosnik JE, Williams HD \& Bundy JG (2013) Metabolite profiling to characterize diseaserelated bacteria: gluconate excretion by Pseudomonas aeruginosa mutants and clinical isolates from cystic fibrosis patients. J Biol Chem 288: 15098-15109.

Bem AE, Velikova N, Pellicer MT, Baarlen P, Marina A \& Wells JM (2015) Bacterial histidine kinases as novel antibacterial drug targets. ACS chemical biology 10: 213-224.

Benit P, Letouze E, Rak M, Aubry L, Burnichon N, Favier J, Gimenez-Roqueplo AP \& Rustin P (2014) Unsuspected task for an old team: succinate, fumarate and other Krebs cycle acids in metabolic remodeling. Biochim Biophys Acta 1837: 1330-1337.

Benkert B, Quack N, Schreiber K, Jaensch L, Jahn D \& Schobert M (2008) Nitrate-responsive NarX-NarL represses arginine-mediated induction of the 
Pseudomonas aeruginosa arginine fermentation $\operatorname{arcDABC}$ operon. Microbiol 154: 3053-3060.

Bernier SP, Ha DG, Khan W, Merritt JH \& O'Toole GA (2011) Modulation of Pseudomonas aeruginosa surface-associated group behaviors by individual amino acids through c-di-GMP signaling. Res Microbiol 162: 680-688.

Bodey GP, Bolivar R, Fainstein V \& Jadeja L (1983) Infections caused by Pseudomonas aeruginosa. Reviews of infectious diseases 5: 279-313.

Borukhov S \& Severinov K (2002) Role of the RNA polymerase sigma subunit in transcription initiation. Res Microbiol 153: 557-562.

Bott M, Meyer M \& Dimroth P (1995) Regulation of anaerobic citrate metabolism in Klebsiella pneumoniae. Mol Microbiol 18: 533-546.

Boucher HW, Talbot GH, Bradley JS, Edwards JE, Gilbert D, Rice LB, Scheld M, Spellberg B \& Bartlett J (2009) Bad bugs, no drugs: no ESKAPE! An update from the Infectious Diseases Society of America. Clinical infectious diseases : an official publication of the Infectious Diseases Society of America 48: 1-12.

Bourret RB, Hess JF, Borkovich KA, Pakula AA \& Simon MI (1989) Protein phosphorylation in chemotaxis and two-component regulatory systems of bacteria. J Biol Chem 264: 7085-7088.

Bowden GH \& Li YH (1997) Nutritional influences on biofilm development. Advances in dental research 11: 81-99.

Bradford MM (1976) A rapid and sensitive method for the quantitation of microgram quantities of protein utilizing the principle of protein-dye binding. Analytical biochemistry 72: 248-254.

Breidenstein EB, de la Fuente-Nunez C \& Hancock RE (2011) Pseudomonas aeruginosa: all roads lead to resistance. Trends Microbiol 19: 419-426.

Brencic A \& Lory S (2009) Determination of the regulon and identification of novel mRNA targets of Pseudomonas aeruginosa RsmA. Mol Microbiol 72: 612632. 
Brencic A, McFarland KA, McManus HR, Castang S, Mogno I, Dove SL \& Lory S (2009) The GacS/GacA signal transduction system of Pseudomonas aeruginosa acts exclusively through its control over the transcription of the RsmY and RsmZ regulatory small RNAs. Mol Microbiol 73: 434-445.

Briard B, Heddergott C \& Latge JP (2016) Volatile Compounds Emitted by Pseudomonas aeruginosa Stimulate Growth of the Fungal Pathogen Aspergillus fumigatus. mBio 7: e00219.

Britton RA \& Young VB (2014) Role of the intestinal microbiota in resistance to colonization by Clostridium difficile. Gastroenterology 146: 1547-1553.

Brocker M, Schaffer S, Mack C \& Bott M (2009) Citrate utilization by Corynebacterium glutamicum is controlled by the CitAB two-component system through positive regulation of the citrate transport genes citH and tctCBA. $J$ Bacteriol 191: 3869-3880.

Browne P, Barret M, O'Gara F \& Morrissey JP (2010) Computational prediction of the $\mathrm{Crc}$ regulon identifies genus-wide and species-specific targets of catabolite repression control in Pseudomonas bacteria. BMC Microbiol 10: 300.

Browning DF \& Busby SJ (2004) The regulation of bacterial transcription initiation. Nat Rev Microbiol 2: 57-65.

Bulla LA (1975) Bacteria as insect pathogens. Annu Rev Microbiol 29: 163-190.

Bullen JJ (1981) The significance of iron in infection. Reviews of infectious diseases 3: 1127-1138.

Burgess RR, Travers AA, Dunn JJ \& Bautz EK (1969) Factor stimulating transcription by RNA polymerase. Nature 221: 43-46.

Burrows LL (2012) Pseudomonas aeruginosa twitching motility: type IV pili in action. Annu Rev Microbiol 66: 493-520.

Busch W \& Saier MH, Jr. (2002) The transporter classification (TC) system, 2002. Crit Rev Biochem Mol Biol 37: 287-337. 
Busch W \& Saier MH, Jr. (2004) The IUBMB-endorsed transporter classification system. Mol Biotechnol 27: 253-262.

Cai W, Wannemuehler Y, Dell'anna G, Nicholson B, Barbieri NL, Kariyawasam S, Feng Y, Logue CM, Nolan LK \& Li G (2013) A novel two-component signaling system facilitates uropathogenic Escherichia coli's ability to exploit abundant host metabolites. PLoS Pathog 9: e1003428.

Caiazza NC, Shanks RM \& O'Toole GA (2005) Rhamnolipids modulate swarming motility patterns of Pseudomonas aeruginosa. J Bacteriol 187: 7351-7361.

Caiazza NC, Merritt JH, Brothers KM \& O'Toole GA (2007) Inverse regulation of biofilm formation and swarming motility by Pseudomonas aeruginosa PA14. $J$ Bacteriol 189: 3603-3612.

Campa M, Bendinelli M, Friedman H \& SpringerLink (Online service) (1993) Pseudomonas aeruginosa as an Opportunistic Pathogen. p.^pp. XX, 419 p. Springer US : Imprint: Springer,, Boston, MA.

Campbell EA, Muzzin O, Chlenov M, Sun JL, Olson CA, Weinman O, TresterZedlitz ML \& Darst SA (2002) Structure of the bacterial RNA polymerase promoter specificity sigma subunit. Molecular cell 9: 527-539.

Campbell JJ \& Stokes FN (1951) Tricarboxylic acid cycle in Pseudomonas aeruginosa. J Biol Chem 190: 853-858.

Campbell JJ, Smith RA \& Eagles BA (1953) A deviation from the conventional tricarboxylic acid cycle in Pseudomonas aeruginosa. Biochim Biophys Acta 11: 594.

Carek PJ, Dickerson LM \& Sack JL (2001) Diagnosis and management of osteomyelitis. American family physician 63: 2413-2420.

Carey BW, Finley LW, Cross JR, Allis CD \& Thompson CB (2015) Intracellular alpha-ketoglutarate maintains the pluripotency of embryonic stem cells. Nature 518: 413-416. 
Cases I, Ussery DW \& de Lorenzo V (2003) The sigma54 regulon (sigmulon) of Pseudomonas putida. Environ Microbiol 5: 1281-1293.

Cashin P, Goldsack L, Hall D \& O'Toole R (2006) Contrasting signal transduction mechanisms in bacterial and eukaryotic gene transcription. FEMS microbiology letters 261: 155-164.

Cepelak I \& Dodig S (2007) Exhaled breath condensate: a new method for lung disease diagnosis. Clin Chem Lab Med 45: 945-952.

Cezairliyan B, Vinayavekhin N, Grenfell-Lee D, Yuen GJ, Saghatelian A \& Ausubel FM (2013) Identification of Pseudomonas aeruginosa phenazines that kill Caenorhabditis elegans. PLoS Pathog 9: e1003101.

Chakravarty A, Carlson JM, Khetani RS \& Gross RH (2007) A novel ensemble learning method for de novo computational identification of DNA binding sites. BMC Bioinform 8: 249.

Chen YT, Chang HY, Lu CL \& Peng HL (2004) Evolutionary analysis of the twocomponent systems in Pseudomonas aeruginosa PAO1. J Mol Evol 59: 725-737.

Choi KH, Kumar A \& Schweizer HP (2006) A 10-min method for preparation of highly electrocompetent Pseudomonas aeruginosa cells: application for DNA fragment transfer between chromosomes and plasmid transformation. J Microbiol Methods 64: 391-397.

Coggan KA \& Wolfgang MC (2012) Global regulatory pathways and cross-talk control Pseudomonas aeruginosa environmental lifestyle and virulence phenotype. Current issues in molecular biology 14: 47-70.

Cohen-Cymberknoh M, Kerem E, Ferkol T \& Elizur A (2013) Airway inflammation in cystic fibrosis: molecular mechanisms and clinical implications. Thorax 68: 1157-1162.

Coleman FT, Mueschenborn S, Meluleni G, Ray C, Carey VJ, Vargas SO, Cannon CL, Ausubel FM \& Pier GB (2003) Hypersusceptibility of cystic fibrosis mice to chronic Pseudomonas aeruginosa oropharyngeal colonization and lung infection. Proc Natl Acad Sci U S A 100: 1949-1954. 
Collier DN, Hager PW \& Phibbs PV, Jr. (1996) Catabolite repression control in the Pseudomonads. Res Microbiol 147: 551-561.

Commichau FM, Forchhammer K \& Stulke J (2006) Regulatory links between carbon and nitrogen metabolism. Curr Opin Microbiol 9: 167-172.

Costerton JW, Stewart PS \& Greenberg EP (1999) Bacterial biofilms: a common cause of persistent infections. Science 284: 1318-1322.

Costerton JW, Cheng KJ, Geesey GG, Ladd TI, Nickel JC, Dasgupta M \& Marrie TJ (1987) Bacterial biofilms in nature and disease. Annu Rev Microbiol 41: 435464.

Crofford OB, Mallard RE, Winton RE, Rogers NL, Jackson JC \& Keller U (1977) Acetone in breath and blood. Trans Am Clin Climatol Assoc 88: 128-139.

Daines C, VanDeVanter D, Khan U, et al. (2014) Serology as a diagnostic tool for predicting initial Pseudomonas aeruginosa acquisition in children with cystic fibrosis. Journal of cystic fibrosis : official journal of the European Cystic Fibrosis Society 13: 542-549.

Dandekar AA, Chugani S \& Greenberg EP (2012) Bacterial quorum sensing and metabolic incentives to cooperate. Science 338: 264-266.

Dandekar T, Snel B, Huynen M \& Bork P (1998) Conservation of gene order: a fingerprint of proteins that physically interact. Trends Biochem Sci 23: 324-328.

Dart JK \& Seal DV (1988) Pathogenesis and therapy of Pseudomonas aeruginosa keratitis. Eye 2 Suppl: S46-55.

Davies DG, Parsek MR, Pearson JP, Iglewski BH, Costerton JW \& Greenberg EP (1998) The involvement of cell-to-cell signals in the development of a bacterial biofilm. Science 280: 295-298.

Davies J (1994) Inactivation of antibiotics and the dissemination of resistance genes. Science 264: 375-382. 
Davies J \& Davies D (2010) Origins and evolution of antibiotic resistance. Microbiol Mol Biol Rev 74: 417-433.

Davies SJ, Golby P, Omrani D, Broad SA, Harrington VL, Guest JR, Kelly DJ \& Andrews SC (1999) Inactivation and regulation of the aerobic $C$ (4)-dicarboxylate transport (dctA) gene of Escherichia coli. J Bacteriol 181: 5624-5635.

de Bentzmann S \& Plesiat P (2011) The Pseudomonas aeruginosa opportunistic pathogen and human infections. Environ Microbiol 13: 1655-1665.

De Kievit TR, Gillis R, Marx S, Brown C \& Iglewski BH (2001) Quorum-sensing genes in Pseudomonas aeruginosa biofilms: their role and expression patterns. Applied and environmental microbiology 67: 1865-1873.

Dent AG, Sutedja TG \& Zimmerman PV (2013) Exhaled breath analysis for lung cancer. Journal of thoracic disease 5 Suppl 5: S540-550.

Deretic V, Leveau JH, Mohr CD \& Hibler NS (1992) In vitro phosphorylation of AlgR, a regulator of mucoidy in Pseudomonas aeruginosa, by a histidine protein kinase and effects of small phospho-donor molecules. Mol Microbiol 6: 27612767.

Deschaght P, Van Daele S, De Baets F \& Vaneechoutte M (2011) PCR and the detection of Pseudomonas aeruginosa in respiratory samples of CF patients. A literature review. Journal of cystic fibrosis : official journal of the European Cystic Fibrosis Society 10: 293-297.

Deziel E, Comeau Y \& Villemur R (2001) Initiation of biofilm formation by Pseudomonas aeruginosa 57RP correlates with emergence of hyperpiliated and highly adherent phenotypic variants deficient in swimming, swarming, and twitching motilities. J Bacteriol 183: 1195-1204.

Deziel E, Lepine F, Milot S \& Villemur R (2003) rhlA is required for the production of a novel biosurfactant promoting swarming motility in Pseudomonas aeruginosa: 3-(3-hydroxyalkanoyloxy)alkanoic acids (HAAs), the precursors of rhamnolipids. Microbiol 149: 2005-2013. 
Dietrich LE, Price-Whelan A, Petersen A, Whiteley M \& Newman DK (2006) The phenazine pyocyanin is a terminal signalling factor in the quorum sensing network of Pseudomonas aeruginosa. Mol Microbiol 61: 1308-1321.

Dodsworth JA, Cady NC \& Leigh JA (2005) 2-Oxoglutarate and the PII homologues Nifl1 and Nifl2 regulate nitrogenase activity in cell extracts of Methanococcus maripaludis. Mol Microbiol 56: 1527-1538.

Doggett RG (1969) Incidence of mucoid Pseudomonas aeruginosa from clinical sources. Appl Microbiol 18: 936-937.

Doucette CD, Schwab DJ, Wingreen NS \& Rabinowitz JD (2011) alphaKetoglutarate coordinates carbon and nitrogen utilization via enzyme I inhibition. Nat Chem Biol 7: 894-901.

Driscoll JA, Brody SL \& Kollef MH (2007) The epidemiology, pathogenesis and treatment of Pseudomonas aeruginosa infections. Drugs 67: 351-368.

Edwards WV, Sando JJ \& Hartline RA (1979) Transport of C5 dicarboxylate compounds by Pseudomonas putida. J Bacteriol 139: 748-754.

Emerson J, Rosenfeld M, McNamara S, Ramsey B \& Gibson RL (2002) Pseudomonas aeruginosa and other predictors of mortality and morbidity in young children with cystic fibrosis. Pediatr Pulmonol 34: 91-100.

Entner N \& Doudoroff M (1952) Glucose and gluconic acid oxidation of Pseudomonas saccharophila. J Biol Chem 196: 853-862.

Essar DW, Eberly L, Hadero A \& Crawford IP (1990) Identification and characterization of genes for a second anthranilate synthase in Pseudomonas aeruginosa: interchangeability of the two anthranilate synthases and evolutionary implications. J Bacteriol 172: 884-900.

Falagas ME \& Bliziotis IA (2007) Pandrug-resistant Gram-negative bacteria: the dawn of the post-antibiotic era? International journal of antimicrobial agents 29: 630-636. 
Fan W \& Almirall J (2014) High-efficiency headspace sampling of volatile organic compounds in explosives using capillary microextraction of volatiles (CMV) coupled to gas chromatography-mass spectrometry (GC-MS). Anal Bioanal Chem 406: 2189-2195.

Fan W, Tsai I \& Qian M (2007) Analysis of 2-aminoacetophenone by directimmersion solid-phase microextraction and gas chromatography-mass spectrometry and its sensory impact in Chardonnay and Pinot gris wines. Food Chemistry 105: 1144-1150.

Feria Bourrellier AB, Valot B, Guillot A, Ambard-Bretteville F, Vidal J \& Hodges M (2010) Chloroplast acetyl-CoA carboxylase activity is 2-oxoglutarate-regulated by interaction of PII with the biotin carboxyl carrier subunit. Proc Natl Acad Sci U S A 107: 502-507.

Fernandez M, Riveros JD, Campos M, Mathee K \& Narasimhan G (2015) Microbial "social networks". BMC genomics 16 Suppl 11: S6.

Figurski DH \& Helinski DR (1979) Replication of an origin-containing derivative of plasmid RK2 dependent on a plasmid function provided in trans. Proc Natl Acad Sci U S A 76: 1648-1652.

Finan TM, Wood JM \& Jordan DC (1981) Succinate transport in Rhizobium leguminosarum. J Bacteriol 148: 193-202.

Finan TM, Wood JM \& Jordan DC (1983) Symbiotic properties of C4-dicarboxylic acid transport mutants of Rhizobium leguminosarum. J Bacteriol 154: 1403-1413.

Fish GW, Hand MM \& Keim WF (1937) Acute Bacterial Endocarditis Due to Pseudomonas Aeruginosa (B. Pyocyaneus): Report of a Case. The American journal of pathology 13: 121-128 121.

Flemming HC, Neu TR \& Wozniak DJ (2007) The EPS matrix: the "house of biofilm cells". J Bacteriol 189: 7945-7947.

Forchhammer K (2008) P(II) signal transducers: novel functional and structural insights. Trends Microbiol 16: 65-72. 
Forchhammer $\mathrm{K}$ (2010) The network of $\mathrm{P}(\mathrm{II})$ signalling protein interactions in unicellular cyanobacteria. Advances in experimental medicine and biology 675: 71-90.

Fraimow H \& Nahra R (2013) Resistant Gram-negative infections. Crit Care Clin 29: 895-921.

Frank LH \& Demoss RD (1959) On the biosynthesis of pyocyanine. J Bacteriol 77: 776-782.

Friedman L \& Kolter R (2004) Genes involved in matrix formation in Pseudomonas aeruginosa PA14 biofilms. Mol Microbiol 51: 675-690.

Frith MC, Saunders NF, Kobe B \& Bailey TL (2008) Discovering sequence motifs with arbitrary insertions and deletions. PLoS Comput Biol 4: e1000071.

Frohman CE, Orten JM \& Smith AH (1951) Chromatographic determination of the acids of the citric acid cycle in tissues. J Biol Chem 193: 277-283.

Furukawa S, Kuchma SL \& O'Toole GA (2006) Keeping their options open: acute versus persistent infections. J Bacteriol 188: 1211-1217.

Galan-Vasquez E, Luna B \& Martinez-Antonio A (2011) The Regulatory Network of Pseudomonas aeruginosa. Microbial informatics and experimentation 1: 3.

Gao R \& Stock AM (2010) Molecular strategies for phosphorylation-mediated regulation of response regulator activity. Curr Opin Microbiol 13: 160-167.

Gaynes R \& Edwards JR (2005) Overview of nosocomial infections caused by gram-negative bacilli. Clin Infect Dis 41: 848-854.

Gaynes R, Edwards JR \& National Nosocomial Infections Surveillance S (2005) Overview of nosocomial infections caused by gram-negative bacilli. Clinical infectious diseases : an official publication of the Infectious Diseases Society of America 41: 848-854. 
Gellatly SL \& Hancock RE (2013) Pseudomonas aeruginosa: new insights into pathogenesis and host defenses. Pathog Dis 67: 159-173.

Giamarellou H (2000) Therapeutic guidelines for Pseudomonas aeruginosa infections. Int $J$ Antimicrob Agents 16: 103-106.

Gisbert JP \& Pajares JM (2004) Review article: 13C-urea breath test in the diagnosis of Helicobacter pylori infection -- a critical review. Alimentary pharmacology \& therapeutics 20: 1001-1017.

Golby P, Davies S, Kelly DJ, Guest JR \& Andrews SC (1999) Identification and characterization of a two-component sensor-kinase and response-regulator system (DcuS-DcuR) controlling gene expression in response to C4dicarboxylates in Escherichia coli. J Bacteriol 181: 1238-1248.

Gooderham WJ \& Hancock RE (2009) Regulation of virulence and antibiotic resistance by two-component regulatory systems in Pseudomonas aeruginosa. FEMS Microbiol Rev 33: 279-294.

Gooderham WJ, Gellatly SL, Sanschagrin F, McPhee JB, Bains M, Cosseau C, Levesque RC \& Hancock RE (2009) The sensor kinase PhoQ mediates virulence in Pseudomonas aeruginosa. Microbiol 155: 699-711.

Goodman AL, Kulasekara B, Rietsch A, Boyd D, Smith RS \& Lory S (2004) A signaling network reciprocally regulates genes associated with acute infection and chronic persistence in Pseudomonas aeruginosa. Dev Cell 7: 745-754.

Gotoh Y, Eguchi Y, Watanabe T, Okamoto S, Doi A \& Utsumi R (2010) Twocomponent signal transduction as potential drug targets in pathogenic bacteria. Curr Opin Microbiol 13: 232-239.

Goudreau PN \& Stock AM (1998) Signal transduction in bacteria: molecular mechanisms of stimulus-response coupling. Curr Opin Microbiol 1: 160-169.

Govan JR \& Nelson JW (1992) Microbiology of lung infection in cystic fibrosis. British medical bulletin 48: 912-930. 
Green MR, Sambrook J \& Sambrook J (2012) Molecular cloning : a laboratory manual. Cold Spring Harbor Laboratory Press, Cold Spring Harbor, N.Y.

Green SK, Schroth MN, Cho JJ, Kominos SK \& Vitanza-jack VB (1974) Agricultural plants and soil as a reservoir for Pseudomonas aeruginosa. Applied microbiology 28: 987-991.

Greenberg EP (2003) Bacterial communication and group behavior. J Clin Invest 112: $1288-1290$.

Groenewold GS, Scott JR \& Rae C (2011) Recovery of phosphonate surface contaminants from glass using a simple vacuum extractor with a solid-phase microextraction fiber. Anal Chim Acta 697: 38-47.

Groisman EA (2001) The pleiotropic two-component regulatory system PhoPPhoQ. J Bacteriol 183: 1835-1842.

Gross R, Arico B \& Rappuoli R (1989) Families of bacterial signal-transducing proteins. Mol Microbiol 3: 1661-1667.

Grzesiak P, Slupecka-Ziemilska M \& Wolinski J (2016) The biological role of aketoglutaric acid in physiological processes and its therapeutic potential. Developmental period medicine 20: 61-67.

Guo Q, Kong W, Jin S, Chen L, Xu Y \& Duan K (2014) PqsR-dependent and PqsR-independent regulation of motility and biofilm formation by PQS in Pseudomonas aeruginosa PAO1. Journal of basic microbiology 54: 633-643.

Guo W, Cai LL, Zou HS, Ma WX, Liu XL, Zou LF, Li YR, Chen XB \& Chen GY (2012) Ketoglutarate transport protein KgtP is secreted through the type III secretion system and contributes to virulence in Xanthomonas oryzae pv. oryzae. Applied and environmental microbiology 78: 5672-5681.

Haas R, Cucchi D, Smith J, Pucino V, Macdougall CE \& Mauro C (2016) Intermediates of Metabolism: From Bystanders to Signalling Molecules. Trends Biochem Sci 41: 460-471. 
Hafiz S \& Oakley CL (1976) Clostridium difficile: isolation and characteristics. J Med Microbiol 9: 129-136.

Hall IC \& O'Toole E (1935) Intestinal flora in new-born infants: with a description of a new pathogenic anaerobe, Bacillus difficilis. Am J Dis Child 49: 390-402.

Hancock RE \& Speert DP (2000) Antibiotic resistance in Pseudomonas aeruginosa: mechanisms and impact on treatment. Drug resistance updates : reviews and commentaries in antimicrobial and anticancer chemotherapy 3: 247255.

Harris KA \& Hartley JC (2003) Development of broad-range 16S rDNA PCR for use in the routine diagnostic clinical microbiology service. J Med Microbio/ 52: 685-691.

Harshey RM (2003) Bacterial motility on a surface: many ways to a common goal. Annu Rev Microbiol 57: 249-273.

Hastie AT, Hingley ST, Higgins ML, Kueppers F \& Shryock T (1986) Rhamnolipid from $P$ seudomonas aeruginosa inactivates mammalian tracheal ciliary axonemes. Cell motility and the cytoskeleton 6: 502-509.

Hauser AR \& Engel JN (1999) Pseudomonas aeruginosa induces type-IIIsecretion-mediated apoptosis of macrophages and epithelial cells. Infect Immun 67: 5530-5537.

Haussler S (2010) Multicellular signalling and growth of Pseudomonas aeruginosa. International journal of medical microbiology : IJMM 300: 544-548.

He W, Miao FJ, Lin DC, Schwandner RT, Wang Z, Gao J, Chen JL, Tian H \& Ling $L$ (2004) Citric acid cycle intermediates as ligands for orphan G-proteincoupled receptors. Nature 429: 188-193.

Hebert SC (2004) Physiology: orphan detectors of metabolism. Nature 429: 143145. 
Heeb S \& Haas D (2001) Regulatory roles of the GacS/GacA two-component system in plant-associated and other gram-negative bacteria. Mol Plant Microbe Interact 14: 1351-1363.

Heurlier K, Denervaud V, Pessi G, Reimmann C \& Haas D (2003) Negative control of quorum sensing by RpoN (sigma54) in Pseudomonas aeruginosa PAO1. J Bacteriol 185: 2227-2235.

Hoch JA \& Silhavy TJ (1995) Two-component signal transduction. ASM Press, Washington, D.C.

Høiby N, Giwercman. B, Jensen. ET, Johansen. HK, Kronborg. G, Pressler. T \& Kharazmi. A (1993) Immune response in cystic fibrosis: helpful or harmful? In H Escobar, C F Baquero, and L Suarez (ed), Clinical ecology of cystic fibrosis Exerpta Medica, Amsterdam, The Netherlands 133-141.

Hopkins MJ \& Macfarlane GT (2002) Changes in predominant bacterial populations in human faeces with age and with Clostridium difficile infection. $J$ Med Microbiol 51: 448-454.

Horton RM, Cai ZL, Ho SN \& Pease LR (1990) Gene splicing by overlap extension: tailor-made genes using the polymerase chain reaction. Biotechniques 8: 528-535.

Huergo LF \& Dixon R (2015) The Emergence of 2-Oxoglutarate as a Master Regulator Metabolite. Microbiology and molecular biology reviews : MMBR 79: 419-435.

Huergo LF, Chandra G \& Merrick M (2013) P(II) signal transduction proteins: nitrogen regulation and beyond. FEMS Microbiol Rev 37: 251-283.

Hugler M, Wirsen CO, Fuchs G, Taylor CD \& Sievert SM (2005) Evidence for autotrophic $\mathrm{CO} 2$ fixation via the reductive tricarboxylic acid cycle by members of the epsilon subdivision of proteobacteria. J Bacteriol 187: 3020-3027.

Hunter GW \& Dweik RA (2008) Applied breath analysis: an overview of the challenges and opportunities in developing and testing sensor technology for human health monitoring in aerospace and clinical applications. J Breath Res 2: 037020 . 
Hunter S, Jones P, Mitchell A, et al. (2012) InterPro in 2011: new developments in the family and domain prediction database. Nucleic Acids Res 40: D306-312.

Janausch IG, Zientz E, Tran QH, Kroger A \& Unden G (2002) C4-dicarboxylate carriers and sensors in bacteria. Biochim Biophys Acta 1553: 39-56.

Jaric M, Segal J, Silva-Herzog E, Schneper L, Mathee K \& Narasimhan G (2013) Better primer design for metagenomics applications by increasing taxonomic distinguishability. BMC Proc 7: S4.

Jennings LK, Storek KM, Ledvina HE, et al. (2015) Pel is a cationic exopolysaccharide that cross-links extracellular DNA in the Pseudomonas aeruginosa biofilm matrix. Proc Natl Acad Sci U S A 112: 11353-11358.

Jensen PO, Bjarnsholt T, Phipps R, et al. (2007) Rapid necrotic killing of polymorphonuclear leukocytes is caused by quorum-sensing-controlled production of rhamnolipid by Pseudomonas aeruginosa. Microbiol 153: 13291338.

Jiang J, Gu BH, Albright LM \& Nixon BT (1989) Conservation between coding and regulatory elements of Rhizobium meliloti and Rhizobium leguminosarum dct genes. J Bacteriol 171: 5244-5253.

Jimenez PN, Koch G, Thompson JA, Xavier KB, Cool RH \& Quax WJ (2012) The multiple signaling systems regulating virulence in Pseudomonas aeruginosa. Microbiology and molecular biology reviews : MMBR 76: 46-65.

Johnson AP \& Woodford N (2013) Global spread of antibiotic resistance: the example of New Delhi metallo-beta-lactamase (NDM)-mediated carbapenem resistance. J Med Microbiol 62: 499-513.

Kalantari A, Derouiche A, Shi L \& Mijakovic I (2015) Serine/threonine/tyrosine phosphorylation regulates DNA binding of bacterial transcriptional regulators. Microbiol 161: 1720-1729.

Karagul-Yuceer Y, Cadwallader KR \& Drake M (2002) Volatile flavor components of stored nonfat dry milk. Journal of agricultural and food chemistry 50: 305-312. 
Kazmierczak BI, Schniederberend M \& Jain R (2015) Cross-regulation of Pseudomonas motility systems: the intimate relationship between flagella, pili and virulence. Curr Opin Microbiol 28: 78-82.

Kelly CP \& LaMont JT (2008) Clostridium difficile--more difficult than ever. N Engl J Med 359: 1932-1940.

Kerby GP (1947) Pseudomonas aeruginosa bacteremia; summary of literature, with report of a case. American journal of diseases of children 74: 610-615.

Kerr JR, Taylor GW, Rutman A, Hoiby N, Cole PJ \& Wilson R (1999)

Pseudomonas aeruginosa pyocyanin and 1-hydroxyphenazine inhibit fungal growth. Journal of clinical pathology 52: 385-387.

Kessler B, de Lorenzo V \& Timmis KN (1992) A general system to integrate lacZ fusions into the chromosomes of gram-negative eubacteria: regulation of the $\mathrm{Pm}$ promoter of the TOL plasmid studied with all controlling elements in monocopy. Mol Gen Genet 233: 293-301.

Kharitonov S, Alving K \& Barnes PJ (1997) Exhaled and nasal nitric oxide measurements: recommendations. The European Respiratory Society Task Force. Eur Respir J 10: 1683-1693.

Kier LD, Weppelman RM \& Ames BN (1979) Regulation of nonspecific acid phosphatase in Salmonella: phoN and phoP genes. J Bacteriol 138: 155-161.

King EO, Ward MK \& Raney DE (1954) Two simple media for the demonstration of pyocyanin and fluorescin. $J$ Lab Clin Med 44: 301-307.

Kirienko NV, Kirienko DR, Larkins-Ford J, Wahlby C, Ruvkun G \& Ausubel FM (2013) Pseudomonas aeruginosa disrupts Caenorhabditis elegans iron homeostasis, causing a hypoxic response and death. Cell host \& microbe 13: 406-416.

Kitten T, Kinscherf TG, McEvoy JL \& Willis DK (1998) A newly identified regulator is required for virulence and toxin production in Pseudomonas syringae. Mol Microbiol 28: 917-929. 
Klausen M, Aaes-Jorgensen A, Molin S \& Tolker-Nielsen T (2003(b)) Involvement of bacterial migration in the development of complex multicellular structures in Pseudomonas aeruginosa biofilms. Mol Microbiol 50: 61-68.

Klausen M, Heydorn A, Ragas P, Lambertsen L, Aaes-Jorgensen A, Molin S \& Tolker-Nielsen T (2003(a)) Biofilm formation by Pseudomonas aeruginosa wild type, flagella and type IV pili mutants. Mol Microbiol 48: 1511-1524.

Knecht H, Neulinger SC, Heinsen FA, et al. (2014) Effects of beta-lactam antibiotics and fluoroquinolones on human gut microbiota in relation to Clostridium difficile associated diarrhea. PLoS One 9: e89417.

Kohler T, Cayrol JM, Ramos JL \& Harayama S (1989) Nucleotide and deduced amino acid sequence of the RpoN sigma-factor of Pseudomonas putida. Nucleic Acids Res 17: 10125.

Kohler T, Curty LK, Barja F, van Delden C \& Pechere JC (2000) Swarming of Pseudomonas aeruginosa is dependent on cell-to-cell signaling and requires flagella and pili. J Bacteriol 182: 5990-5996.

Kong KF, Jayawardena SR, Indulkar SD, Del Puerto A, Koh CL, Hoiby N \& Mathee K (2005) Pseudomonas aeruginosa AmpR is a global transcriptional factor that regulates expression of AmpC and PoxB beta-lactamases, proteases, quorum sensing, and other virulence factors. Antimicrob Agents Chemother 49: $4567-4575$.

Kong KF, Jayawardena SR, Del Puerto A, Wiehlmann L, Laabs U, Tummler B \& Mathee K (2005) Characterization of poxB, a chromosomal-encoded Pseudomonas aeruginosa oxacillinase. Gene 358: 82-92.

Kornberg HL (1966) Anaplerotic sequences and their role in metabolism. Essays Biochem 2:1-31.

Koutsokera A, Kostikas K, Nicod LP \& Fitting JW (2013) Pulmonary biomarkers in COPD exacerbations: a systematic review. Respiratory research 14: 111.

Kownatzki R, Tummler B \& Doring G (1987) Rhamnolipid of Pseudomonas aeruginosa in sputum of cystic fibrosis patients. Lancet 1: 1026-1027. 
Krebs HA (1940) The citric acid cycle and the Szent-Gyorgyi cycle in pigeon breast muscle. Biochem J 34: 775-779.

Kuchma SL, Brothers KM, Merritt JH, Liberati NT, Ausubel FM \& O'Toole GA (2007) BifA, a cyclic-Di-GMP phosphodiesterase, inversely regulates biofilm formation and swarming motility by Pseudomonas aeruginosa PA14. J Bacteriol 189: 8165-8178.

Kumari H, Balasubramanian D, Zincke D \& Mathee K (2014) Role of Pseudomonas aeruginosa AmpR on beta-lactam and non-beta-lactam transient cross-resistance upon pre-exposure to subinhibitory concentrations of antibiotics. J Med Microbiol 63: 544-555.

Kunz DA, Chen JL \& Pan G (1998) Accumulation of alpha-keto acids as essential components in cyanide assimilation by Pseudomonas fluorescens NCIMB 11764. Applied and environmental microbiology 64: 4452-4459.

Kwon DH \& Lu CD (2006) Polyamines induce resistance to cationic peptide, aminoglycoside, and quinolone antibiotics in Pseudomonas aeruginosa PAO1. Antimicrobial agents and chemotherapy 50: 1615-1622.

Lamont IL, Konings AF \& Reid DW (2009) Iron acquisition by Pseudomonas aeruginosa in the lungs of patients with cystic fibrosis. Biometals : an international journal on the role of metal ions in biology, biochemistry, and medicine 22: 53-60.

Lancien M, Gadal P \& Hodges M (2000) Enzyme redundancy and the importance of 2-oxoglutarate in higher plant ammonium assimilation. Plant physiology 123: 817-824.

Lappin-Scott H, Burton S \& Stoodley P (2014) Revealing a world of biofilms--the pioneering research of Bill Costerton. Nat Rev Microbiol 12: 781-787.

Larson HE \& Welch A (1993) In vitro and in vivo characterisation of resistance to colonisation with Clostridium difficile. J Med Microbiol 38: 103-108.

Larson HE, Price AB, Honour P \& Borriello SP (1978) Clostridium difficile and the aetiology of pseudomembranous colitis. Lancet 1: 1063-1066. 
Laskowski MA \& Kazmierczak BI (2006) Mutational analysis of RetS, an unusual sensor kinase-response regulator hybrid required for Pseudomonas aeruginosa virulence. Infect Immun 74: 4462-4473.

Laskowski MA, Osborn E \& Kazmierczak BI (2004) A novel sensor kinaseresponse regulator hybrid regulates type III secretion and is required for virulence in Pseudomonas aeruginosa. Mol Microbiol 54: 1090-1103.

Lau GW, Hassett DJ, Ran H \& Kong F (2004) The role of pyocyanin in Pseudomonas aeruginosa infection. Trends in molecular medicine 10: 599-606.

Ledizet M, Murray TS, Puttagunta S, Slade MD, Quagliarello VJ \& Kazmierczak $\mathrm{BI}$ (2012) The ability of virulence factor expression by Pseudomonas aeruginosa to predict clinical disease in hospitalized patients. PloS one 7: e49578.

Lee PC, Stopford CM, Svenson AG \& Rietsch A (2010) Control of effector export by the Pseudomonas aeruginosa type III secretion proteins PcrG and PcrV. Mol Microbiol 75: 924-941.

Leech AJ, Sprinkle A, Wood L, Wozniak DJ \& Ohman DE (2008) The NtrC family regulator AlgB, which controls alginate biosynthesis in mucoid Pseudomonas aeruginosa, binds directly to the algD promoter. J Bacteriol 190: 581-589.

Lehninger AL, Nelson DL \& Cox MM (2013) Lehninger principles of biochemistry. W.H. Freeman, New York.

Leigh JA \& Dodsworth JA (2007) Nitrogen regulation in bacteria and archaea. Annu Rev Microbiol 61: 349-377.

Lemire J, Milandu Y, Auger C, Bignucolo A, Appanna VP \& Appanna VD (2010) Histidine is a source of the antioxidant, alpha-ketoglutarate, in Pseudomonas fluorescens challenged by oxidative stress. FEMS microbiology letters 309: 170177.

Lessie TG \& Phibbs PV, Jr. (1984) Alternative pathways of carbohydrate utilization in pseudomonads. Annu Rev Microbiol 38: 359-388. 
Letouze E, Martinelli C, Loriot C, et al. (2013) SDH mutations establish a hypermethylator phenotype in paraganglioma. Cancer cell 23: 739-752.

Li W \& Lu CD (2007) Regulation of carbon and nitrogen utilization by CbrAB and NtrBC two-component systems in Pseudomonas aeruginosa. J Bacteriol 189: 5413-5420.

Liang H, Li L, Dong Z, Surette MG \& Duan K (2008) The YebC family protein PA0964 negatively regulates the Pseudomonas aeruginosa quinolone signal system and pyocyanin production. J Bacteriol 190: 6217-6227.

Linares JF, Gustafsson I, Baquero F \& Martinez JL (2006) Antibiotics as intermicrobial signaling agents instead of weapons. Proc Natl Acad Sci U S A 103: 19484-19489.

Lister PD, Wolter DJ \& Hanson ND (2009) Antibacterial-resistant Pseudomonas aeruginosa: clinical impact and complex regulation of chromosomally encoded resistance mechanisms. Clin Microbiol Rev 22: 582-610.

Liu P (1952) Utilization of carbohydrates by Pseudomonas aeruginosa. $J$ Bacteriol 64: 773-781.

Loenarz C \& Schofield CJ (2008) Expanding chemical biology of 2-oxoglutarate oxygenases. Nat Chem Biol 4: 152-156.

Long LH \& Halliwell B (2011) Artefacts in cell culture: alpha-ketoglutarate can scavenge hydrogen peroxide generated by ascorbate and epigallocatechin gallate in cell culture media. Biochem Biophys Res Commun 406: 20-24.

Loomis WF, Jr. \& Magasanik B (1967) Glucose-lactose diauxie in Escherichia coli. J Bacteriol 93: 1397-1401.

Lukat GS, McCleary WR, Stock AM \& Stock JB (1992) Phosphorylation of bacterial response regulator proteins by low molecular weight phospho-donors. Proc Natl Acad Sci U S A 89: 718-722. 
Lundgren BR, Villegas-Penaranda LR, Harris JR, Mottern AM, Dunn DM, Boddy CN \& Nomura CT (2014) Genetic analysis of the assimilation of C5-dicarboxylic acids in Pseudomonas aeruginosa PAO1. J Bacteriol 196: 2543-2551.

Lyczak JB, Cannon CL \& Pier GB (2000) Establishment of Pseudomonas aeruginosa infection: lessons from a versatile opportunist. Microbes Infect 2: 1051-1060.

Lyczak JB, Cannon CL \& Pier GB (2002) Lung infections associated with cystic fibrosis. Clin Microbiol Rev 15: 194-222.

Lyczak JB, Cannon CL \& Pier GB (2002) Lung infections associated with cystic fibrosis. Clin Microbiol Rev 15: 194-222.

Ma L, Wang S, Wang D, Parsek MR \& Wozniak DJ (2012) The roles of biofilm matrix polysaccharide Psl in mucoid Pseudomonas aeruginosa biofilms. FEMS Immunol Med Microbiol 65: 377-380.

Ma S, Selvaraj U, Ohman DE, Quarless R, Hassett DJ \& Wozniak DJ (1998) Phosphorylation-independent activity of the response regulators AlgB and AlgR in promoting alginate biosynthesis in mucoid Pseudomonas aeruginosa. $\mathrm{J}$ Bacteriol 180: 956-968.

Macfarlane EL, Kwasnicka A \& Hancock RE (2000) Role of Pseudomonas aeruginosa PhoP-phoQ in resistance to antimicrobial cationic peptides and aminoglycosides. Microbiol 146 ( Pt 10): 2543-2554.

Mahajan-Miklos S, Tan MW, Rahme LG \& Ausubel FM (1999) Molecular mechanisms of bacterial virulence elucidated using a Pseudomonas aeruginosaCaenorhabditis elegans pathogenesis model. Cell 96: 47-56.

Mailloux RJ, Puiseux-Dao S \& Appanna VD (2009) Alpha-ketoglutarate abrogates the nuclear localization of HIF-1alpha in aluminum-exposed hepatocytes. Biochimie 91: 408-415.

Makimura K, Murayama SY \& Yamaguchi H (1994) Detection of a wide range of medically important fungi by the polymerase chain reaction. $J$ Med Microbiol 40: 358-364. 
Manefield M, de Nys R, Kumar N, Read R, Givskov M, Steinberg P \& Kjelleberg $S$ (1999) Evidence that halogenated furanones from Delisea pulchra inhibit acylated homoserine lactone (AHL)-mediated gene expression by displacing the AHL signal from its receptor protein. Microbiology 145: 283-291.

Manfredi R, Nanetti A, Ferri M \& Chiodo F (2000) Pseudomonas spp. complications in patients with HIV disease: an eight-year clinical and microbiological survey. Eur J Epidemiol 16: 111-118.

Mann S (1966) Uber den Geruchsstoff von Pseudomonas aeruginosa. Archiv fur Mikrobiologie 54: 184-190.

Marchler-Bauer A, Derbyshire MK, Gonzales NR, et al. (2015) CDD: NCBI's conserved domain database. Nucleic Acids Res 43: D222-226.

Markou P \& Apidianakis Y (2014) Pathogenesis of intestinal Pseudomonas aeruginosa infection in patients with cancer. Frontiers in cellular and infection microbiology 3: 115.

Mascher T (2013) Signaling diversity and evolution of extracytoplasmic function (ECF) sigma factors. Curr Opin Microbiol 16: 148-155.

Mascher T, Helmann JD \& Unden G (2006) Stimulus perception in bacterial signal-transducing histidine kinases. Microbiology and molecular biology reviews : MMBR 70: 910-938.

Masseret E, Boudeau J, Colombel JF, Neut C, Desreumaux P, Joly B, Cortot A \& Darfeuille-Michaud A (2001) Genetically related Escherichia coli strains associated with Crohn's disease. Gut 48: 320-325.

Mathee K, Ciofu O, Sternberg C, et al. (1999) Mucoid conversion of Pseudomonas aeruginosa by hydrogen peroxide: a mechanism for virulence activation in the cystic fibrosis lung. Microbiology 145 1349-1357.

Mathee K, Narasimhan G, Valdes C, et al. (2008) Dynamics of Pseudomonas aeruginosa genome evolution. Proc Natl Acad Sci U S A 105: 3100-3105. 
Mavrodi DV, Bonsall RF, Delaney SM, Soule MJ, Phillips G \& Thomashow LS (2001) Functional analysis of genes for biosynthesis of pyocyanin and phenazine-1-carboxamide from Pseudomonas aeruginosa PAO1. J Bacteriol 183: 6454-6465.

McPhee JB, Lewenza S \& Hancock RE (2003) Cationic antimicrobial peptides activate a two-component regulatory system, PmrA-PmrB, that regulates resistance to polymyxin $\mathrm{B}$ and cationic antimicrobial peptides in Pseudomonas aeruginosa. Mol Microbiol 50: 205-217.

McPhee JB, Bains M, Winsor G, Lewenza S, Kwasnicka A, Brazas MD, Brinkman FS \& Hancock RE (2006) Contribution of the PhoP-PhoQ and PmrA$\mathrm{PmrB}$ two-component regulatory systems to $\mathrm{Mg} 2+-$-induced gene regulation in Pseudomonas aeruginosa. J Bacteriol 188: 3995-4006.

Merrick MJ (1993) In a class of its own--the RNA polymerase sigma factor sigma 54 (sigma N). Mol Microbiol 10: 903-909.

Merritt JH, Brothers KM, Kuchma SL \& O'Toole GA (2007) SadC reciprocally influences biofilm formation and swarming motility via modulation of exopolysaccharide production and flagellar function. J Bacteriol 189: 8154-8164.

Merritt JH, Ha DG, Cowles KN, Lu W, Morales DK, Rabinowitz J, Gitai Z \& O'Toole GA (2010) Specific control of Pseudomonas aeruginosa surfaceassociated behaviors by two c-di-GMP diguanylate cyclases. mBio 1.

Meyer JM (2000) Pyoverdines: pigments, siderophores and potential taxonomic markers of fluorescent Pseudomonas species. Archives of microbiology 174: $135-142$.

Meyer JM, Neely A, Stintzi A, Georges C \& Holder IA (1996) Pyoverdin is essential for virulence of Pseudomonas aeruginosa. Infect Immun 64: 518-523.

Miekisch W, Schubert JK \& Noeldge-Schomburg GF (2004) Diagnostic potential of breath analysis--focus on volatile organic compounds. Clin Chim Acta 347: 2539. 
Mikkelsen H, Sivaneson M \& Filloux A (2011) Key two-component regulatory systems that control biofilm formation in Pseudomonas aeruginosa. Environ Microbiol 13: 1666-1681.

Miller SI, Kukral AM \& Mekalanos JJ (1989) A two-component regulatory system (phoP phoQ) controls Salmonella typhimurium virulence. Proc Natl Acad Sci U S A 86: 5054-5058.

Mitrophanov AY \& Groisman EA (2008) Signal integration in bacterial twocomponent regulatory systems. Genes \& development 22: 2601-2611.

Mittal R, Lisi CV, Gerring R, et al. (2015) Current concepts in the pathogenesis and treatment of chronic suppurative otitis media. J Med Microbiol 64: 11031116.

Moet GJ, Jones RN, Biedenbach DJ, Stilwell MG \& Fritsche TR (2007) Contemporary causes of skin and soft tissue infections in North America, Latin America, and Europe: report from the SENTRY Antimicrobial Surveillance Program (1998-2004). Diagn Microbiol Infect Dis 57: 7-13.

Moreno R, Ruiz-Manzano A, Yuste L \& Rojo F (2007) The Pseudomonas putida Crc global regulator is an RNA binding protein that inhibits translation of the AlkS transcriptional regulator. Mol Microbiol 64: 665-675.

Morett E \& Segovia L (1993) The sigma 54 bacterial enhancer-binding protein family: mechanism of action and phylogenetic relationship of their functional domains. J Bacteriol 175: 6067-6074.

Moskowitz SM, Ernst RK \& Miller SI (2004) PmrAB, a two-component regulatory system of $P$ seudomonas aeruginosa that modulates resistance to cationic antimicrobial peptides and addition of aminoarabinose to lipid A. J Bacteriol 186: 575-579.

Murakami KS \& Darst SA (2003) Bacterial RNA polymerases: the wholo story. Current opinion in structural biology 13: 31-39.

Nealson KH \& Hastings JW (1979) Bacterial bioluminescence: its control and ecological significance. Microbiol Rev 43: 496-518. 
Niesters HG (2002) Clinical virology in real time. Journal of clinical virology: the official publication of the Pan American Society for Clinical Virology 25 Suppl 3: S3-12.

Ninfa AJ \& Jiang P (2005) PIl signal transduction proteins: sensors of alphaketoglutarate that regulate nitrogen metabolism. Curr Opin Microbiol 8: 168-173.

Nishijyo T, Haas D \& Itoh Y (2001) The CbrA-CbrB two-component regulatory system controls the utilization of multiple carbon and nitrogen sources in Pseudomonas aeruginosa. Mol Microbiol 40: 917-931.

Nostro A, Sudano Roccaro A, Bisignano G, Marino A, Cannatelli MA, Pizzimenti FC, Cioni PL, Procopio F \& Blanco AR (2007) Effects of oregano, carvacrol and thymol on Staphylococcus aureus and Staphylococcus epidermidis biofilms. $J$ Med Microbiol 56: 519-523.

O'Toole GA (2011) Microtiter dish biofilm formation assay. Journal of visualized experiments : JoVE.

Ohman DE \& Chakrabarty AM (1981) Genetic mapping of chromosomal determinants for the production of the exopolysaccharide alginate in a Pseudomonas aeruginosa cystic fibrosis isolate. Infect Immun 33: 142-148.

Okkotsu Y, Little AS \& Schurr MJ (2014) The Pseudomonas aeruginosa AlgZR two-component system coordinates multiple phenotypes. Frontiers in cellular and infection microbiology 4: 82.

Oliver A, Canton R, Campo P, Baquero F \& Blazquez J (2000) High frequency of hypermutable Pseudomonas aeruginosa in cystic fibrosis lung infection. Science 288: $1251-1254$.

Ondrako JM \& Ornston LN (1980) Biological distribution and physiological role of the beta-ketoadipate transport system. Journal of general microbiology 120: 199209.

Osuna J, Soberon X \& Morett E (1997) A proposed architecture for the central domain of the bacterial enhancer-binding proteins based on secondary structure prediction and fold recognition. Protein Sci 6: 543-555. 
Overbeek R, Fonstein M, D'Souza M, Pusch GD \& Maltsev N (1999) The use of gene clusters to infer functional coupling. Proc Natl Acad Sci U S A 96: 28962901.

Owen OE, Kalhan SC \& Hanson RW (2002) The key role of anaplerosis and cataplerosis for citric acid cycle function. J Biol Chem 277: 30409-30412.

Paget MS \& Helmann JD (2003) The sigma70 family of sigma factors. Genome Biol 4: 203.

Palmer KL, Aye LM \& Whiteley M (2007) Nutritional cues control Pseudomonas aeruginosa multicellular behavior in cystic fibrosis sputum. J Bacteriol 189: 80798087.

Palmer KL, Mashburn LM, Singh PK \& Whiteley M (2005) Cystic fibrosis sputum supports growth and cues key aspects of Pseudomonas aeruginosa physiology. J Bacteriol 187: 5267-5277.

Parkinson JS (1993) Signal transduction schemes of bacteria. Cell 73: 857-871.

Parks QM, Young RL, Poch KR, Malcolm KC, Vasil ML \& Nick JA (2009) Neutrophil enhancement of Pseudomonas aeruginosa biofilm development: human F-actin and DNA as targets for therapy. J Med Microbiol 58: 492-502.

Payne DJ (1993) Metallo-beta-lactamases--a new therapeutic challenge. J Med Microbiol 39: 93-99.

Penketh A, Pitt T, Roberts D, Hodson ME \& Batten JC (1983) The relationship of phenotype changes in Pseudomonas aeruginosa to the clinical condition of patients with cystic fibrosis. The American review of respiratory disease 127: 605-608.

Perez-Rueda E, Janga SC \& Martinez-Antonio A (2009) Scaling relationship in the gene content of transcriptional machinery in bacteria. Molecular bioSystems 5: 1494-1501.

Pessi G, Williams F, Hindle Z, Heurlier K, Holden MT, Camara M, Haas D \& Williams P (2001) The global posttranscriptional regulator RsmA modulates 
production of virulence determinants and $\mathrm{N}$-acylhomoserine lactones in Pseudomonas aeruginosa. J Bacteriol 183: 6676-6683.

Petrova OE \& Sauer K (2009) A novel signaling network essential for regulating Pseudomonas aeruginosa biofilm development. PLoS Pathog 5: e1000668.

Petrova OE, Schurr JR, Schurr MJ \& Sauer K (2012) Microcolony formation by the opportunistic pathogen Pseudomonas aeruginosa requires pyruvate and pyruvate fermentation. Mol Microbiol 86: 819-835.

Pier GB, Matthews WJ, Jr. \& Eardley DD (1983) Immunochemical characterization of the mucoid exopolysaccharide of Pseudomonas aeruginosa. $J$ Infect Dis 147: 494-503.

Pierson LS, 3rd \& Pierson EA (2010) Metabolism and function of phenazines in bacteria: impacts on the behavior of bacteria in the environment and biotechnological processes. Applied microbiology and biotechnology 86: 16591670.

Poole K \& McKay GA (2003) Iron acquisition and its control in Pseudomonas aeruginosa: many roads lead to Rome. Frontiers in bioscience : a journal and virtual library 8: d661-686.

Potvin E, Sanschagrin F \& Levesque RC (2008) Sigma factors in Pseudomonas aeruginosa. FEMS Microbiol Rev 32: 38-55.

Potvin E, Lehoux DE, Kukavica-Ibrulj I, Richard KL, Sanschagrin F, Lau GW \& Levesque RC (2003) In vivo functional genomics of Pseudomonas aeruginosa for high-throughput screening of new virulence factors and antibacterial targets. Environ Microbiol 5: 1294-1308.

Prevatt AR, Sedwick JD, Gajewski BJ \& Antonelli PJ (2004) Hearing loss with semicircular canal transection and Pseudomonas aeruginosa otitis media. Otolaryngol Head Neck Surg 131: 248-252.

Price-Whelan A, Dietrich LE \& Newman DK (2006) Rethinking 'secondary' metabolism: physiological roles for phenazine antibiotics. Nat Chem Biol 2: 7178. 
Pritt B, O'Brien L \& Winn W (2007) Mucoid Pseudomonas in cystic fibrosis. Am J Clin Pathol 128: 32-34.

Prost LR, Daley ME, Bader MW, Klevit RE \& Miller SI (2008) The PhoQ histidine kinases of Salmonella and Pseudomonas spp. are structurally and functionally different: evidence that $\mathrm{pH}$ and antimicrobial peptide sensing contribute to mammalian pathogenesis. Mol Microbiol 69: 503-519.

Qin X, Emerson J, Stapp J, Stapp L, Abe P \& Burns JL (2003) Use of real-time PCR with multiple targets to identify Pseudomonas aeruginosa and other nonfermenting gram-negative bacilli from patients with cystic fibrosis. Journal of clinical microbiology 41: 4312-4317.

Rabinowitz JD \& Silhavy TJ (2013) Systems biology: metabolite turns master regulator. Nature 500: 283-284.

Raghavan V \& Groisman EA (2010) Orphan and hybrid two-component system proteins in health and disease. Curr Opin Microbiol 13: 226-231.

Rahme LG, Stevens EJ, Wolfort SF, Shao J, Tompkins RG \& Ausubel FM (1995) Common virulence factors for bacterial pathogenicity in plants and animals.

Science 268: 1899-1902.

Rahme LG, Ausubel FM, Cao H, et al. (2000) Plants and animals share functionally common bacterial virulence factors. Proc Natl Acad Sci U S A 97: 8815-8821.

Ramos J-L (2004) Pseudomonas. Kluwer Academic/Plenum, Boston.

Ran H, Hassett DJ \& Lau GW (2003) Human targets of Pseudomonas aeruginosa pyocyanin. Proc Natl Acad Sci U S A 100: 14315-14320.

Rashid MH \& Kornberg A (2000) Inorganic polyphosphate is needed for swimming, swarming, and twitching motilities of Pseudomonas aeruginosa. Proc Natl Acad Sci U S A 97: 4885-4890.

Ratjen F \& Doring G (2003) Cystic fibrosis. Lancet 361: 681-689. 
Reid CJ \& Poole PS (1998) Roles of DctA and DctB in signal detection by the dicarboxylic acid transport system of Rhizobium leguminosarum. J Bacteriol 180: 2660-2669.

Reszka KJ, O'Malley Y, McCormick ML, Denning GM \& Britigan BE (2004) Oxidation of pyocyanin, a cytotoxic product from Pseudomonas aeruginosa, by microperoxidase 11 and hydrogen peroxide. Free radical biology \& medicine 36 : 1448-1459.

Rice LB (2008) Federal funding for the study of antimicrobial resistance in nosocomial pathogens: no ESKAPE. The Journal of infectious diseases 197: 1079-1081.

Rietsch A, Vallet-Gely I, Dove SL \& Mekalanos JJ (2005) ExsE, a secreted regulator of type III secretion genes in Pseudomonas aeruginosa. Proc Natl Acad Sci U S A 102: 8006-8011.

Ritchings BW, Almira EC, Lory S \& Ramphal R (1995) Cloning and phenotypic characterization of fle $S$ and fleR, new response regulators of Pseudomonas aeruginosa which regulate motility and adhesion to mucin. Infect Immun 63: 4868-4876.

Rodrigue A, Quentin Y, Lazdunski A, Mejean V \& Foglino M (2000) Twocomponent systems in Pseudomonas aeruginosa: why so many? Trends Microbiol 8: 498-504.

Romano AH \& Conway T (1996) Evolution of carbohydrate metabolic pathways. Res Microbiol 147: 448-455.

Ronson CW, Astwood PM, Nixon BT \& Ausubel FM (1987) Deduced products of C4-dicarboxylate transport regulatory genes of Rhizobium leguminosarum are homologous to nitrogen regulatory gene products. Nucleic Acids Res 15: 79217934.

Ronson CW, Nixon BT, Albright LM \& Ausubel FM (1987) Rhizobium meliloti ntrA $(r p o N)$ gene is required for diverse metabolic functions. $J$ Bacteriol 169: 24242431. 
Saier MH, Jr., Tran CV \& Barabote RD (2006) TCDB: the Transporter Classification Database for membrane transport protein analyses and information. Nucleic Acids Res 34: D181-186.

Sambrook J \& Russell DW (2001) Molecular cloning : a laboratory manual. Cold Spring Harbor Laboratory Press, Cold Spring Harbor, N.Y.

Sandre RM \& Shafran SD (1996) Infective endocarditis: review of 135 cases over 9 years. Clinical infectious diseases : an official publication of the Infectious Diseases Society of America 22: 276-286.

Sarbach C, Stevens P, Whiting J, Puget P, Humbert M, Cohen-Kaminsky S \& Postaire $E$ (2013) Evidence of endogenous volatile organic compounds as biomarkers of diseases in alveolar breath. Annales pharmaceutiques francaises 71: 203-215.

Sauer K, Camper AK, Ehrlich GD, Costerton JW \& Davies DG (2002) Pseudomonas aeruginosa displays multiple phenotypes during development as a biofilm. J Bacteriol 184: 1140-1154.

Sauer K, Cullen MC, Rickard AH, Zeef LA, Davies DG \& Gilbert P (2004) Characterization of nutrient-induced dispersion in Pseudomonas aeruginosa PAO1 biofilm. J Bacteriol 186: 7312-7326.

Sautter R, Ramos D, Schneper L, et al. (2012) A complex multilevel attack on Pseudomonas aeruginosa algT/U expression and algT/U activity results in the loss of alginate production. Gene 498: 242-253.

Savelev SU, Perry JD, Bourke SJ, Jary H, Taylor R, Fisher AJ, Corris PA, Petrie M \& De Soyza A (2011) Volatile biomarkers of Pseudomonas aeruginosa in cystic fibrosis and noncystic fibrosis bronchiectasis. Lett Appl Microbiol 52: 610613.

Schaber JA, Hammond A, Carty NL, Williams SC, Colmer-Hamood JA, Burrowes BH, Dhevan V, Griswold JA \& Hamood AN (2007) Diversity of biofilms produced by quorum-sensing-deficient clinical isolates of Pseudomonas aeruginosa. $J$ Med Microbiol 56: 738-748. 
Schmarr HG, Ganss S, Sang W \& Potouridis T (2007) Analysis of 2aminoacetophenone in wine using a stable isotope dilution assay and multidimensional gas chromatography-mass spectrometry. Journal of chromatography A 1150: 78-84.

Schneper L, Maricic. N \& Mathee. K (2011) Anti-quorum sensing, anti-bacterial, and immunomodulatory properties of Panax ginseng. Intl J Biomed Pharma Sci 6: 11-24.

Schreiber K, Krieger R, Benkert B, Eschbach M, Arai H, Schobert M \& Jahn D (2007) The anaerobic regulatory network required for Pseudomonas aeruginosa nitrate respiration. J Bacteriol 189: 4310-4314.

Schreiber M, Res I \& Matter A (2009) Protein kinases as antibacterial targets. Current opinion in cell biology 21: 325-330.

Schultz J, Milpetz F, Bork P \& Ponting CP (1998) SMART, a simple modular architecture research tool: identification of signaling domains. Proc Natl Acad Sci U S A 95: 5857-5864.

Schulz S, Eckweiler D, Bielecka A, Nicolai T, Franke R, Dotsch A, Hornischer K, Bruchmann S, Duvel J \& Haussler S (2015) Elucidation of sigma factorassociated networks in Pseudomonas aeruginosa reveals a modular architecture with limited and function-specific crosstalk. PLoS Pathog 11: e1004744.

Schweizer HP \& Hoang TT (1995) An improved system for gene replacement and xylE fusion analysis in Pseudomonas aeruginosa. Gene 158: 15-22.

Scott-Thomas AJ, Syhre M, Pattemore PK, Epton M, Laing R, Pearson J \& Chambers ST (2010) 2-Aminoacetophenone as a potential breath biomarker for Pseudomonas aeruginosa in the cystic fibrosis lung. BMC Pulm Med 10: 56.

Seol W \& Shatkin AJ (1991) Escherichia coli kgtP encodes an alphaketoglutarate transporter. Proc Natl Acad Sci U S A 88: 3802-3806.

Seol W \& Shatkin AJ (1992) Site-directed mutants of Escherichia coli alphaketoglutarate permease (KgtP). Biochemistry 31: 3550-3554. 
Seol W \& Shatkin AJ (1993) Membrane topology model of Escherichia coli alphaketoglutarate permease by phoA fusion analysis. J Bacteriol 175: 565-567.

Sevvana M, Vijayan V, Zweckstetter M, Reinelt S, Madden DR, Herbst-Irmer R, Sheldrick GM, Bott M, Griesinger C \& Becker S (2008) A ligand-induced switch in the periplasmic domain of sensor histidine kinase CitA. Journal of molecular biology 377: 512-523.

Shanks RM, Kadouri DE, MacEachran DP \& O'Toole GA (2009) New yeast recombineering tools for bacteria. Plasmid 62: 88-97.

Shanks RM, Caiazza NC, Hinsa SM, Toutain CM \& O'Toole GA (2006) Saccharomyces cerevisiae-based molecular tool kit for manipulation of genes from Gram-negative bacteria. Applied and environmental microbiology 72: 50275036.

Shen J, Meldrum A \& Poole K (2002) FpvA receptor involvement in pyoverdine biosynthesis in Pseudomonas aeruginosa. J Bacteriol 184: 3268-3275.

Shrout JD, Chopp DL, Just CL, Hentzer M, Givskov M \& Parsek MR (2006) The impact of quorum sensing and swarming motility on Pseudomonas aeruginosa biofilm formation is nutritionally conditional. Mol Microbiol 62: 1264-1277.

Siegmund I \& Wagner F (1991) New method for detecting rhamnolipids excreted by Pseudomonas species during growth on mineral agar. Biotechnology Techniques 5: 265-268.

Sievert DM, Ricks P, Edwards JR, et al. (2013) Antimicrobial-resistant pathogens associated with healthcare-associated infections: summary of data reported to the National Healthcare Safety Network at the Centers for Disease Control and Prevention, 2009-2010. Infection control and hospital epidemiology 34: 1-14.

Silby MW, Winstanley C, Godfrey SA, Levy SB \& Jackson RW (2011) Pseudomonas genomes: diverse and adaptable. FEMS Microbiol Rev 35: 652680.

Silver LL (2007) Multi-targeting by monotherapeutic antibacterials. Nat Rev Drug Discov 6: 41-55. 
Silver LL (2011) Challenges of antibacterial discovery. Clin Microbiol Rev 24: 71109.

Sing VO \& Schroth MN (1977) Bacteria--plant cell surface interactions: active immobilization of saprophytic bacteria in plant leaves. Science 197: 759-761.

Skaar EP (2010) The battle for iron between bacterial pathogens and their vertebrate hosts. PLoS Pathog 6: e1000949.

Smith RS \& Iglewski BH (2003) P. aeruginosa quorum-sensing systems and virulence. Curr Opin Microbiol 6: 56-60.

Song Z, Wu H, Ciofu O, Kong KF, Hoiby N, Rygaard J, Kharazmi A \& Mathee K (2003) Pseudomonas aeruginosa alginate is refractory to Th1 immune response and impedes host immune clearance in a mouse model of acute lung infection. $J$ Med Microbiol 52: 731-740.

Sonnleitner E, Abdou L \& Haas D (2009) Small RNA as global regulator of carbon catabolite repression in Pseudomonas aeruginosa. Proc Natl Acad Sci U S A 106: 21866-21871.

Sorde R, Pahissa A \& Rello J (2011) Management of refractory Pseudomonas aeruginosa infection in cystic fibrosis. Infection and drug resistance 4: 31-41.

Sousa AM \& Pereira MO (2014) Pseudomonas aeruginosa Diversification during Infection Development in Cystic Fibrosis Lungs-A Review. Pathogens 3: 680703.

Sriramulu DD, Lunsdorf H, Lam JS \& Romling U (2005) Microcolony formation: a novel biofilm model of Pseudomonas aeruginosa for the cystic fibrosis lung. $J$ Med Microbiol 54: 667-676.

Stapper AP, Narasimhan G, Ohman DE, Barakat J, Hentzer M, Molin S, Kharazmi A, Hoiby N \& Mathee K (2004) Alginate production affects Pseudomonas aeruginosa biofilm development and architecture, but is not essential for biofilm formation. J Med Microbiol 53: 679-690. 
Stephen AM \& Cummings JH (1980) The microbial contribution to human faecal mass. J Med Microbiol 13: 45-56.

Stevens EJ, Ryan CM, Friedberg JS, Barnhill RL, Yarmush ML \& Tompkins RG (1994) A quantitative model of invasive Pseudomonas infection in burn injury. The Journal of burn care \& rehabilitation 15: 232-235.

Stewart V \& Bledsoe PJ (2008) Substitutions at auxiliary operator O3 enhance repression by nitrate-responsive regulator $\mathrm{NarL}$ at synthetic lac control regions in Escherichia coli K-12. J Bacteriol 190: 428-433.

Stock AM, Robinson VL \& Goudreau PN (2000) Two-component signal transduction. Annu Rev Biochem 69: 183-215.

Stock JB, Ninfa AJ \& Stock AM (1989) Protein phosphorylation and regulation of adaptive responses in bacteria. Microbiol Rev 53: 450-490.

Stover CK, Pham XQ, Erwin AL, et al. (2000) Complete genome sequence of Pseudomonas aeruginosa PAO1, an opportunistic pathogen. Nature 406: 959964.

Strateva T \& Yordanov D (2009) Pseudomonas aeruginosa - a phenomenon of bacterial resistance. J Med Microbiol 58: 1133-1148.

Stulke J \& Hillen W (2000) Regulation of carbon catabolism in Bacillus species. Annu Rev Microbiol 54: 849-880.

Suh SJ, Runyen-Janecky LJ, Maleniak TC, Hager P, MacGregor CH, ZielinskiMozny NA, Phibbs PV, Jr. \& West SE (2002) Effect of vfr mutation on global gene expression and catabolite repression control of Pseudomonas aeruginosa. Microbiol 148: 1561-1569.

Sun Y, Karmakar M, Roy S, et al. (2010) TLR4 and TLR5 on corneal macrophages regulate Pseudomonas aeruginosa keratitis by signaling through MyD88-dependent and -independent pathways. J Immunol 185: 4272-4283. 
Takase H, Nitanai H, Hoshino K \& Otani T (2000) Impact of siderophore production on Pseudomonas aeruginosa infections in immunosuppressed mice. Infect Immun 68: 1834-1839.

Talbot GH, Bradley J, Edwards JE, Jr., Gilbert D, Scheld M \& Bartlett JG (2006) Bad bugs need drugs: an update on the development pipeline from the Antimicrobial Availability Task Force of the Infectious Diseases Society of America. Clin Infect Dis 42: 657-668.

Tang DJ, He YQ, Feng JX, He BR, Jiang BL, Lu GT, Chen B \& Tang JL (2005) Xanthomonas campestris pv. campestris possesses a single gluconeogenic pathway that is required for virulence. J Bacteriol 187: 6231-6237.

Tanigawa R, Shirokane M, Maeda Si S, Omata T, Tanaka K \& Takahashi H (2002) Transcriptional activation of NtcA-dependent promoters of Synechococcus sp. PCC 7942 by 2-oxoglutarate in vitro. Proc Natl Acad Sci U S A 99: 4251-4255.

Tarifa A \& Almirall JR (2015) Fast detection and characterization of organic and inorganic gunshot residues on the hands of suspects by CMV-GC-MS and LIBS. Science \& justice : journal of the Forensic Science Society 55: 168-175.

Tatke G, Kumari H, Silva-Herzog E, Ramirez L \& Mathee K (2015) Pseudomonas aeruginosa MifS-MifR Two-Component System Is Specific for alphaKetoglutarate Utilization. PloS one 10: e0129629.

Theriot CM, Koenigsknecht MJ, Carlson PE, Jr., Hatton GE, Nelson AM, Li B, Huffnagle GB, J ZL \& Young VB (2014) Antibiotic-induced shifts in the mouse gut microbiome and metabolome increase susceptibility to Clostridium difficile infection. Nat Commun 5: 3114.

Thony B \& Hennecke H (1989) The $-24 /-12$ promoter comes of age. FEMS Microbiol Rev 5: 341-357.

Tokonami N, Morla L, Centeno G, et al. (2013) alpha-Ketoglutarate regulates acid-base balance through an intrarenal paracrine mechanism. The Journal of clinical investigation 123: 3166-3171. 
Tolker-Nielsen T (2014) Pseudomonas aeruginosa biofilm infections: from molecular biofilm biology to new treatment possibilities. APMIS Supplementum 151.

Tommasi R, Brown DG, Walkup GK, Manchester JI \& Miller AA (2015) ESKAPEing the labyrinth of antibacterial discovery. Nature reviews Drug discovery 14: 529-542.

Toutain CM, Zegans ME \& O'Toole GA (2005) Evidence for two flagellar stators and their role in the motility of Pseudomonas aeruginosa. J Bacteriol 187: 771777.

Tramper-Stranders GA, van der Ent CK \& Wolfs TF (2005) Detection of Pseudomonas aeruginosa in patients with cystic fibrosis. Journal of cystic fibrosis : official journal of the European Cystic Fibrosis Society 4 Suppl 2: 37-43.

Tramper-Stranders GA, van der Ent CK, Slieker MG, Terheggen-Lagro SW, Teding van Berkhout F, Kimpen JL \& Wolfs TF (2006) Diagnostic value of serological tests against $P$ seudomonas aeruginosa in a large cystic fibrosis population. Thorax 61: 689-693.

Tummler B, Wiehlmann L, Klockgether J \& Cramer N (2014) Advances in understanding Pseudomonas. F1000prime reports 6: 9.

Turnbaugh PJ, Ley RE, Hamady M, Fraser-Liggett CM, Knight R \& Gordon JI (2007) The human microbiome project. Nature 449: 804-810.

Turner KH, Everett J, Trivedi U, Rumbaugh KP \& Whiteley M (2014) Requirements for Pseudomonas aeruginosa acute burn and chronic surgical wound infection. PLoS Genetics 10: e1004518.

Tynecka Z, Korona-Glowniak I \& Los R (2001) 2-Oxoglutarate transport system in Staphylococcus aureus. Archives of microbiology 176: 143-150.

Ueda A \& Wood TK (2009) Connecting quorum sensing, c-di-GMP, pel polysaccharide, and biofilm formation in Pseudomonas aeruginosa through tyrosine phosphatase TpbA (PA3885). PLoS Pathog 5: e1000483. 
Valderrey AD, Pozuelo MJ, Jimenez PA, Macia MD, Oliver A \& Rotger R (2010) Chronic colonization by Pseudomonas aeruginosa of patients with obstructive lung diseases: cystic fibrosis, bronchiectasis, and chronic obstructive pulmonary disease. Diagn Microbiol Infect Dis 68: 20-27.

Valentini M \& Lapouge K (2013) Catabolite repression in Pseudomonas aeruginosa $\mathrm{PAO} 1$ regulates the uptake of $\mathrm{C} 4$-dicarboxylates depending on succinate concentration. Environ Microbiol 15: 1707-1716.

Valentini M, Storelli N \& Lapouge K (2011) Identification of C(4)-dicarboxylate transport systems in Pseudomonas aeruginosa PAO1. J Bacteriol 193: 43074316.

Van Alst NE, Picardo KF, Iglewski BH \& Haidaris CG (2007) Nitrate sensing and metabolism modulate motility, biofilm formation, and virulence in Pseudomonas aeruginosa. Infect Immun 75: 3780-3790.

Vandenende CS, Vlasschaert M \& Seah SY (2004) Functional characterization of an aminotransferase required for pyoverdine siderophore biosynthesis in Pseudomonas aeruginosa PAO1. J Bacteriol 186: 5596-5602.

Varughese KI (2002) Molecular recognition of bacterial phosphorelay proteins. Curr Opin Microbiol 5: 142-148.

Vazquez-Bermudez MF, Herrero A \& Flores E (2002) 2-Oxoglutarate increases the binding affinity of the NtcA (nitrogen control) transcription factor for the Synechococcus gInA promoter. FEBS letters 512: 71-74.

Vento S, Cainelli F \& Temesgen Z (2008) Lung infections after cancer chemotherapy. The Lancet Oncology 9: 982-992.

Ventre I, Goodman AL, Vallet-Gely I, Vasseur P, Soscia C, Molin S, Bleves S, Lazdunski A, Lory S \& Filloux A (2006) Multiple sensors control reciprocal expression of Pseudomonas aeruginosa regulatory RNA and virulence genes. Proc Natl Acad Sci U S A 103: 171-176.

Visca P, Imperi F \& Lamont IL (2007) Pyoverdine siderophores: from biogenesis to biosignificance. Trends Microbiol 15: 22-30. 
Waite RD, Paccanaro A, Papakonstantinopoulou A, Hurst JM, Saqi M, Littler E \& Curtis MA (2006) Clustering of Pseudomonas aeruginosa transcriptomes from planktonic cultures, developing and mature biofilms reveals distinct expression profiles. BMC Genomics 7: 162.

Walker TS, Bais HP, Deziel E, Schweizer HP, Rahme LG, Fall R \& Vivanco JM (2004) Pseudomonas aeruginosa-plant root interactions. Pathogenicity, biofilm formation, and root exudation. Plant physiology 134: 320-331.

Wang M, Schaefer AL, Dandekar AA \& Greenberg EP (2015) Quorum sensing and policing of Pseudomonas aeruginosa social cheaters. Proc Natl Acad Sci U S A 112: 2187-2191.

Wanner BL \& Wilmes-Riesenberg MR (1992) Involvement of phosphotransacetylase, acetate kinase, and acetyl phosphate synthesis in control of the phosphate regulon in Escherichia coli. J Bacteriol 174: 2124-2130.

Watson RJ (1990) Analysis of the C4-dicarboxylate transport genes of Rhizobium meliloti: nucleotide sequence and deduced products of $\operatorname{dct} A, \operatorname{dctB}$, and $\operatorname{dct} D$. Mol Plant Microbe Interact 3: 174-181.

Williams P (2007) Quorum sensing, communication and cross-kingdom signalling in the bacterial world. Microbiology 153: 3923-3938.

Williams P \& Camara M (2009) Quorum sensing and environmental adaptation in Pseudomonas aeruginosa: a tale of regulatory networks and multifunctional signal molecules. Curr Opin Microbiol 12: 182-191.

Wilson M (1996) Susceptibility of oral bacterial biofilms to antimicrobial agents. $J$ Med Microbiol 44: 79-87.

Winsor GL, Lam DK, Fleming L, Lo R, Whiteside MD, Yu NY, Hancock RE \& Brinkman FS (2011) Pseudomonas Genome Database: improved comparative analysis and population genomics capability for Pseudomonas genomes. Nucleic Acids Res 39: D596-600.

Winstanley C, O'Brien S \& Brockhurst MA (2016) Pseudomonas aeruginosa Evolutionary Adaptation and Diversification in Cystic Fibrosis Chronic Lung Infections. Trends Microbiol 24: 327-337. 
Woese CR \& Fox GE (1977) Phylogenetic structure of the prokaryotic domain: the primary kingdoms. Proc Natl Acad Sci U S A 74: 5088-5090.

Woese CR, Magrum LJ \& Fox GE (1978) Archaebacteria. J Mol Evol 11: 245251.

Wolff JA, MacGregor CH, Eisenberg RC \& Phibbs PV, Jr. (1991) Isolation and characterization of catabolite repression control mutants of Pseudomonas aeruginosa PAO. J Bacteriol 173: 4700-4706.

Wolfgang MC, Lee VT, Gilmore ME \& Lory S (2003) Coordinate regulation of bacterial virulence genes by a novel adenylate cyclase-dependent signaling pathway. Dev Cell 4: 253-263.

Wood LF, Leech AJ \& Ohman DE (2006) Cell wall-inhibitory antibiotics activate the alginate biosynthesis operon in Pseudomonas aeruginosa: Roles of sigma (AlgT) and the AlgW and Prc proteases. Mol Microbiol 62: 412-426.

Workentine ML, Sibley CD, Glezerson B, Purighalla S, Norgaard-Gron JC, Parkins MD, Rabin HR \& Surette MG (2013) Phenotypic heterogeneity of Pseudomonas aeruginosa populations in a cystic fibrosis patient. PLoS One 8: e60225.

Wozniak DJ \& Ohman DE (1991) Pseudomonas aeruginosa AlgB, a twocomponent response regulator of the $\mathrm{NtrC}$ family, is required for $\operatorname{alg} D$ transcription. J Bacteriol 173: 1406-1413.

Wurtzel O, Yoder-Himes DR, Han K, Dandekar AA, Edelheit S, Greenberg EP, Sorek R \& Lory S (2012) The single-nucleotide resolution transcriptome of Pseudomonas aeruginosa grown in body temperature. PLoS Pathog 8: e1002945.

Xu J, Moore JE, Murphy PG, Millar BC \& Elborn JS (2004) Early detection of Pseudomonas aeruginosa--comparison of conventional versus molecular (PCR) detection directly from adult patients with cystic fibrosis (CF). Annals of clinical microbiology and antimicrobials 3: 21.

Yahr TL \& Greenberg EP (2004) The genetic basis for the commitment to chronic versus acute infection in Pseudomonas aeruginosa. Molecular cell 16: 497-498. 
Yahr TL \& Wolfgang MC (2006) Transcriptional regulation of the Pseudomonas aeruginosa type III secretion system. Mol Microbiol 62: 631-640.

Yamamoto H, Murata M \& Sekiguchi J (2000) The CitST two-component system regulates the expression of the Mg-citrate transporter in Bacillus subtilis. Mol Microbiol 37: 898-912.

Yarosh OK, Charles TC \& Finan TM (1989) Analysis of C4-dicarboxylate transport genes in Rhizobium meliloti. Mol Microbiol 3: 813-823.

Yeo SG, Park DC, Hong SM, Cha CI \& Kim MG (2007) Bacteriology of chronic suppurative otitis media--a multicenter study. Acta Otolaryngol 127: 1062-1067.

Yeung AT, Bains M \& Hancock RE (2011) The sensor kinase CbrA is a global regulator that modulates metabolism, virulence, and antibiotic resistance in Pseudomonas aeruginosa. J Bacteriol 193: 918-931.

Yeung AT, Torfs EC, Jamshidi F, Bains M, Wiegand I, Hancock RE \& Overhage $\mathrm{J}$ (2009) Swarming of Pseudomonas aeruginosa is controlled by a broad spectrum of transcriptional regulators, including MetR. J Bacteriol 191: 55925602.

Youn JW, Jolkver E, Kramer R, Marin K \& Wendisch VF (2009) Characterization of the dicarboxylate transporter DctA in Corynebacterium glutamicum. $J$ Bacteriol 191: 5480-5488.

Zdzisinska B, Zurek A \& Kandefer-Szerszen M (2016) Alpha-Ketoglutarate as a Molecule with Pleiotropic Activity: Well-Known and Novel Possibilities of Therapeutic Use. Archivum immunologiae et therapiae experimentalis.

Zhang S \& Bryant DA (2011) The tricarboxylic acid cycle in cyanobacteria. Science 334: 1551-1553.

Zhang XX \& Rainey PB (2008) Dual involvement of CbrAB and NtrBC in the regulation of histidine utilization in Pseudomonas fluorescens SBW25. Genetics 178: 185-195. 
Zhou YF, Nan B, Nan J, Ma Q, Panjikar S, Liang YH, Wang Y \& Su XD (2008) C4-dicarboxylates sensing mechanism revealed by the crystal structures of DctB sensor domain. Journal of molecular biology 383: 49-61.

Zolfaghar I, Angus AA, Kang PJ, To A, Evans DJ \& Fleiszig SM (2005) Mutation of retS, encoding a putative hybrid two-component regulatory protein in Pseudomonas aeruginosa, attenuates multiple virulence mechanisms. Microbes Infect 7: 1305-1316.

Zscheppank C, Wiegand HL, Lenzen C, Wingender J \& Telgheder U (2014) Investigation of volatile metabolites during growth of Escherichia coli and Pseudomonas aeruginosa by needle trap-GC-MS. Anal Bioanal Chem 406: 6617-6628. 
VITA

GORAKH DIGAMBAR TATKE

\section{EDUCATION AND EXPERIENCE}

2010-2016

Doctoral Candidate in Biology

Florida International University

Miami, FL

2006-2008

M.S. Biotechnology

Bangalore University

Bangalore, India

2002-2006

B.S. Biotechnology

University of Mumbai

Mumbai, India

2015 - Dissertation Year Fellowship, University Graduate School, Florida

2016 International University

$201522^{\text {nd }}$ Prize for the Best Oral Presentation at the $17^{\text {th }}$ Biomedical and Comparative Immunology Symposium, Florida International University

$2015 \quad 2^{\text {nd }}$ Prize for the Best Oral presentation at the $17^{\text {th }}$ Annual Biology Research Symposium, Florida International University

2014 Best Oral Presentation Award at the $16^{\text {th }}$ Annual Biomedical and Comparative Immunology Symposium, Florida International University

2014 NIH MBRS RISE Biomedical Research Initiative (BRI) Student Summer Research Award, Florida International University

2013 NIH MBRS RISE Biomedical Research Initiative (BRI) Student Summer Research Award, Florida International University

\begin{tabular}{|c|c|c|}
\hline Major Professor & Department & Project Title \\
\hline Dr. Narasimhan & $\begin{array}{l}\text { Computer } \\
\text { Science }\end{array}$ & Microbiome and Metabolome Studies \\
\hline Dr. Almirall & $\begin{array}{l}\text { Chemistry \& } \\
\text { Biochemistry }\end{array}$ & $\begin{array}{l}\text { Rapid Detection of Mucoid Pseudomonas } \\
\text { aeruginosa using Microextraction of Volatiles }\end{array}$ \\
\hline Dr. Liuzzi & $\begin{array}{l}\text { Dietetics \& } \\
\text { Nutrition }\end{array}$ & Developing Caenorhabditis elegans assay \\
\hline
\end{tabular}



Dr. Sakhrat
Electrical
Nuclear Magnetic Resonance and Magneto-
Engineering
Electric Nanoparticles to Detect Bacteria

\section{PUBLICATIONS}

O. Weeks, G. Tatke, K. Mathee, C. Espinal (2016). Report: $6^{\text {th }}$ International Conference on Global Health. http://ghc.fiu.edu/?page_id=3491

K. Mathee, L.L.Silver and G. Tatke (2015). $70^{\text {th }}$ Anniversary Collection of the Society of General Microbiology: Journal of Medical Microbiology. Journal of Medical Microbiology 1457-61.

G. Tatke, H. Kumari, E. Silva-Herzog, Lourdes Ramirez, and K. Mathee (2015). Pseudomonas aeruginosa MifS-MifR two-competent system is specific for aketoglutarate utilization. PloS one e0129629

Balasubramanian D, S.K. Murugapiran, E. Silva-Herzog, L. Schneper, X. Yang, G. Tatke, G. Narasimhan, and K. Mathee (2013). Transcriptional regulatory network in Pseudomonas aeruginosa. Bacterial Gene Regulation and Transcriptional Networks. 195-248. M. Babu (Ed.), Caister Academic Press.

American Society of Microbiology: Microbe (2016). G. Tatke, S. Mustafi, M.A. Barbieri and K. Mathee. a-ketoglutatate mediated regulation of Pseudomonas aeruginosa pathogenesis by MifS-MifR Two-Component System

Cold Spring Harbor Laboratory: Microbial Pathogenesis and Host Response (2015). G. Tatke, S. Mustafi, M.A. Barbieri and K. Mathee. a-Ketoglutarate dependent regulation of Pseudomonas aeruginosa virulence by the MifS-MifR two-competent system

$17^{\text {th }}$ Biomedical and Comparative Immunology Symposium, Florida International University (2015). G.Tatke, H. Kumari, E. Silva-Herzog, L. Ramirez, S. Mustafi, M.A. Barbieri and K. Mathee. Functional analysis to characterize the role of MifSR two-component system proteins in regulating Pseudomonas aeruginosa.

$17^{\text {th }}$ Annual Biology Research Symposium, Florida International University (2015). G. Tatke, H. Kumari, E. Silva-Herzog, L. Ramirez and K. Mathee. Characterizing the role of MifSR two-component system proteins in regulating Pseudomonas aeruginosa pathogenesis.

Annual Conference for Society for General Microbiology, Liverpool, UK (2014). G. Tatke, H. Kumari, E. Silva-Herzog, L. Ramirez and K. Mathee. Characterizing the role of MifSR two-component system proteins in regulating Pseudomonas aeruginosa metabolism.

$16^{\text {th }}$ Biomedical and Comparative Immunology Symposium, Florida International University (2014). G. Tatke, H. Kumari, E. Silva-Herzog, L. Ramirez and K. Mathee. 